UNIVERSIDAD POLITÉNICA DE MADRID

ESCUELA TÉCNICA SUPERIOR DE INGENIERÍA Y SISTEMAS DE TELECOMUNICACIÓN

DEPARTAMENTO DE INGENIÉRIA TELEMÁTICA Y ELECTRÓNICA

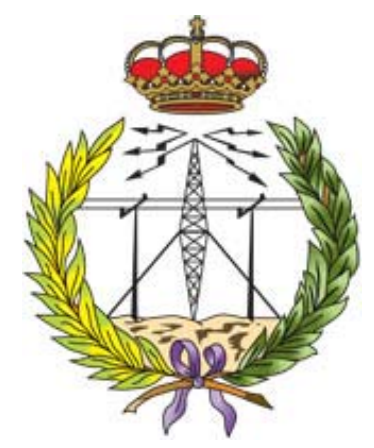

\title{
UN MODELO SOSTENIBLE PARA LA \\ GESTIÓN DE DECISIONES DE DISEÑO EN ARQUITECTURA SOFTWARE
}

\section{TESIS DOCTORAL}

\author{
Carlos Carrillo Sánchez \\ Master Sistemas Telemáticos e Informáticos \\ DIRECTOR \\ Dr. Rafael Capilla Sevilla \\ Doctor por la Universidad Rey Juan Carlos
}





\section{TRIBUNAL}

Tribunal nombrado por el Magfco. Y Excmo Sr. Rector de la Universidad Politécnica de Madrid, el día de de 2017.

Presidente: Dr. D. Juan Carlos Dueñas López

Secretario: Dra. Dña. Jennifer Pérez Benedí

Vocal 1: Dr. D. Carlos Enrique Cuesta Quintero

Vocal 2: Dra. Dña. Elena María Navarro Martín

Vocal 3: Dr. D. Daniel Rodríguez García

Suplente: Dr. D. Juan José Pantrigo Fernández

Suplente: Dr. D. Víctor Manuel López Jaquero

Realizado el acto de lectura y defensa de la Tesis Doctoral el día de de 2017 en la Escuela Técnica Superior de Ingeniería y Sistemas de Telecomunicación de la Universidad Politécnica de Madrid, y habiendo obtenido una calificación de

EL PRESIDENTE

LOS VOCALES

LA SECRETARIA 



\section{Agradecimientos:}

A Rafa por su tesón y paciencia, a Mónica por su sonrisa, a Ana por su enorme y constante apoyo, y a los que no menciono, gracias. 



\begin{abstract}
For more than three decades, software architectures have been the cornerstone of software design. Traditionally, a software architecture has been understood as a set of components and connectors that represent a design solution for a software problem.

However, since 2004, the scientific community has begun to consider architectures as the result of a set of design decisions, in which architectural knowledge is based not only on design patterns and architectural styles, but also on considering design decisions as first-class elements. It is therefore important to capture such decisions to avoid knowledge vaporization.

In this sense, design decisions play a relevant role not only in capturing the knowledge of the experts but also to facilitate the processes of evolution and maintenance of the system. One of the key aspects in maintaining the software architecture and the underlying decisions is to define and manage the amount of architectural knowledge to be captured. In this sense, one of the recent trends is to measure the sustainability of systems and hence their architecture. Because most of the metrics that measure system quality are code-driven, specific metrics are needed to measure the sustainability of software architectures. However, there is a lack of metrics that allow us to measure how sustainable is a set of design decisions.
\end{abstract}

For these reasons, the main objective of this Doctoral Thesis is to investigate models and metrics that allow us to evaluate the sustainability of a set of design decisions in order to determine how sustainable a software architecture can be. 


\section{Resumen}

Desde hace más de tres décadas las arquitecturas software constituyen la piedra angular del diseño software. Tradicionalmente, una arquitectura software se entiende como un conjunto de componentes y conectores que representan una solución de diseño para un problema software.

Sin embargo, desde 2004, la comunidad científica ha empezado a considerar a las arquitecturas como el resultado de un conjunto de decisiones de diseño, en la que el conocimiento arquitectónico no se basa en los patrones de diseño y estilos arquitectónicos, sino también en considerar a las decisiones de diseño como elementos de primera clase. Por ello, resulta importante capturar dichas decisiones para evitar la pérdida del conocimiento.

En este sentido, las decisiones de diseño juegan un papel relevante no solo en la captura del conocimiento de los expertos sino también para facilitar los procesos de evolución y mantenimiento del sistema. Uno de los aspectos clave a la hora de mantener la arquitectura software y las decisiones subyacentes es definir y gestionar la cantidad de conocimiento arquitectónico a capturar. En este sentido, una de las tendencias recientes es medir la sostenibilidad de los sistemas y por ende de su arquitectura. Debido a que la mayoría de las métricas que miden la calidad de los sistemas están enfocadas a código, resulta necesario contar con métricas específicas para medir la sostenibilidad de las arquitecturas software. Sin embargo, existe una carencia de métricas que nos permitan medir como de sostenible es un conjunto de decisiones de diseño.

Por estos motivos, el objetivo principal de este Tesis Doctoral va a ser investigar en modelos y métricas que nos permitan evaluar la sostenibilidad de un conjunto de decisiones de diseño con el fin de determinar cómo de sostenible puede ser una arquitectura software. 


\section{Índice}

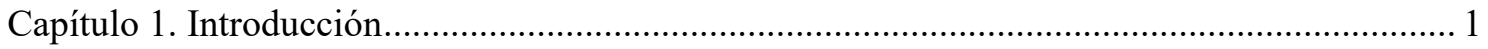

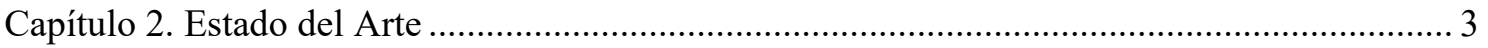

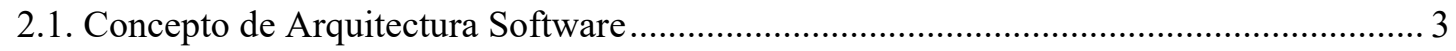

2.2. Decisiones de Diseño en Arquitectura Software............................................................... 5

2.2.1. Caracterización de decisiones de diseño ............................................................... 7

2.2.2. Meta-modelos para la representación de decisiones de diseño .................................. 8

2.3. Herramientas para la Captura de Decisiones de Diseño ...................................................... 12

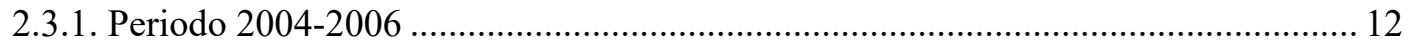

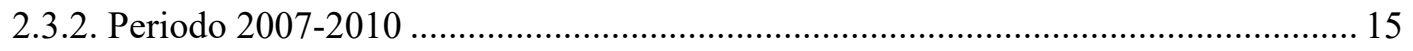

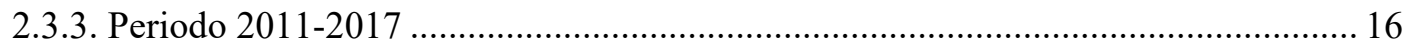

2.4. Trazabilidad en Decisiones de Diseño ....................................................................... 19

2.5. Evolución Sostenible en Arquitecturas Software........................................................... 21

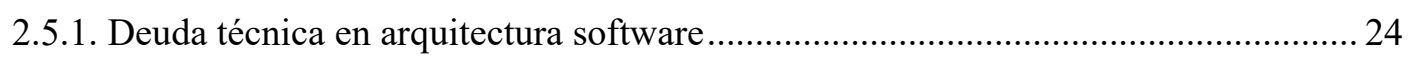

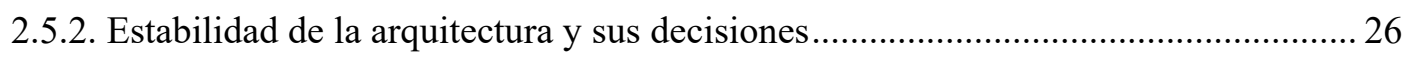

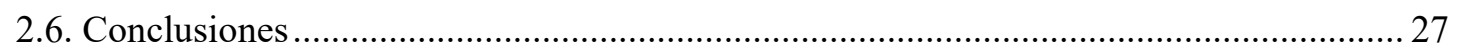

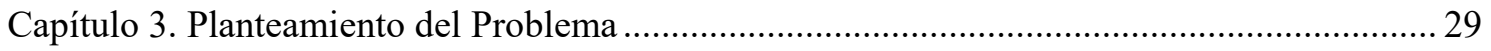

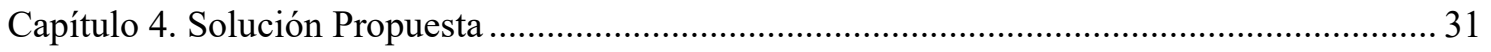

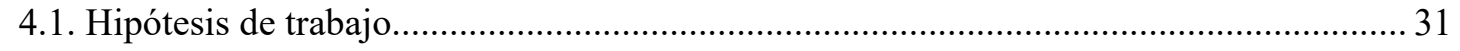

4.2. Modelo Sostenible para la Gestión de Decisiones de Diseño ............................................ 32

4.2.1. Meta-modelo para la gestión sostenible de decisiones de diseño .............................. 33

4.3. Criterios para un Conocimiento Arquitectónico Sostenible.............................................. 42

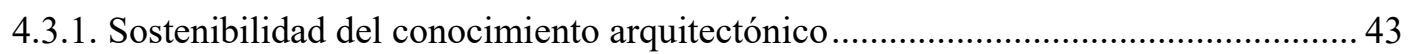

4.3.2. Sostenibilidad en la evolución del conocimiento arquitectónico ............................... 44

4.3.3. Taxonomía para estimar la sostenibilidad del conocimiento arquitectónico ............. 46

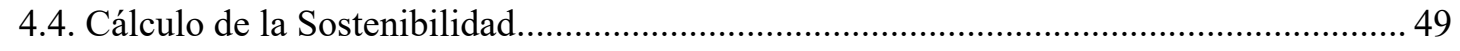

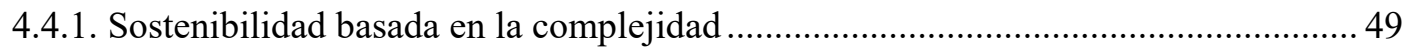

4.4.2. Sostenibilidad basada en el esfuerzo de capturar decisiones.................................... 52

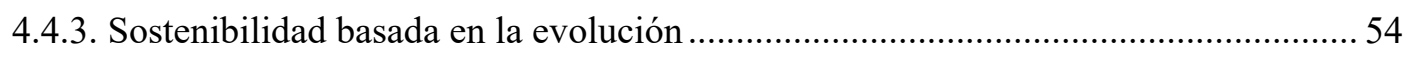

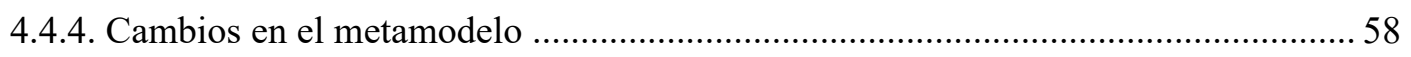

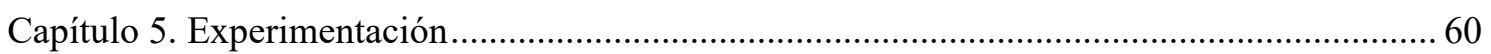

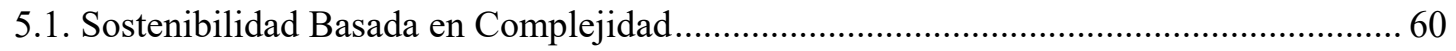

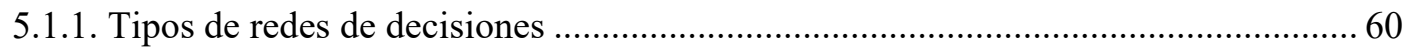

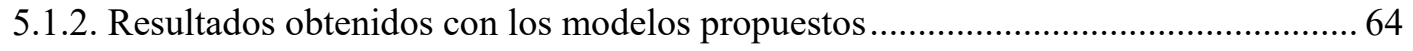

5.1.3. Experimentación con redes de tipo plataformas basadas en servicios....................... 68 
5.1.4. Resultados con redes de decisiones complejas ....................................................... 77

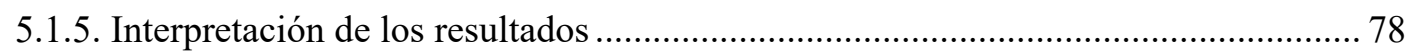

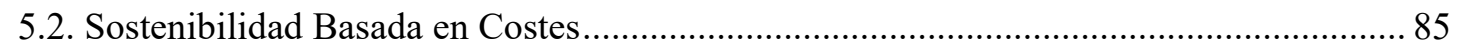

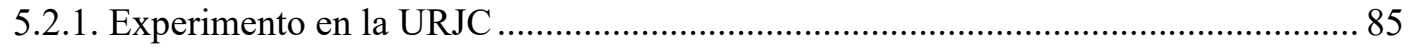

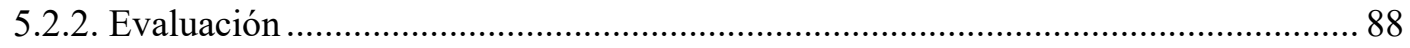

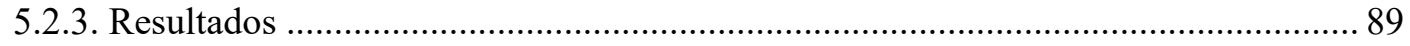

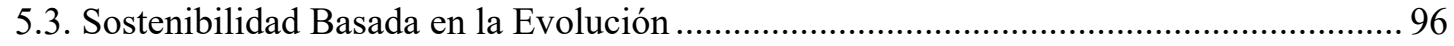

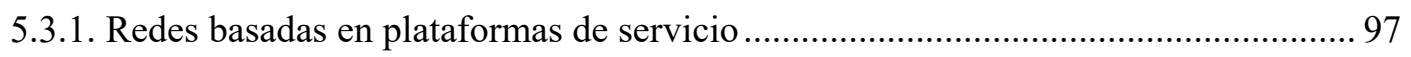

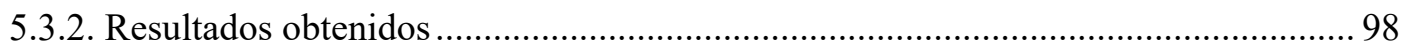

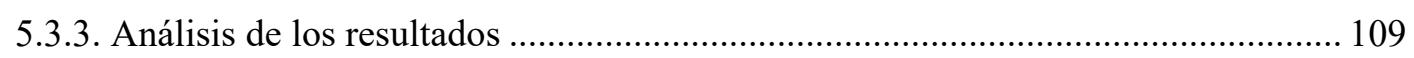

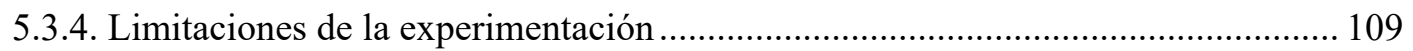

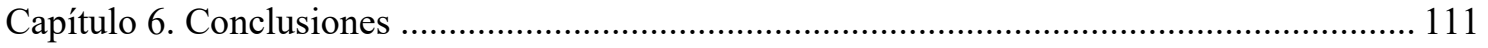

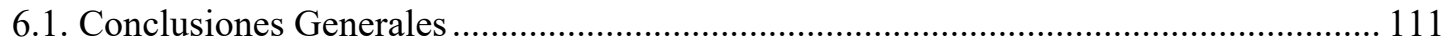

6.2. Conclusiones de la Experimentación ............................................................................ 111

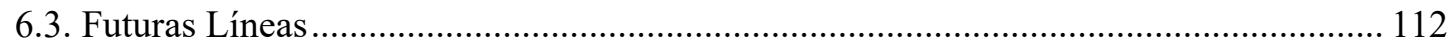

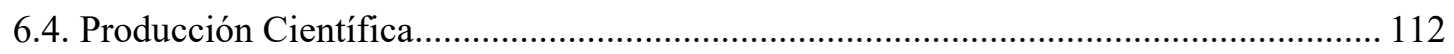

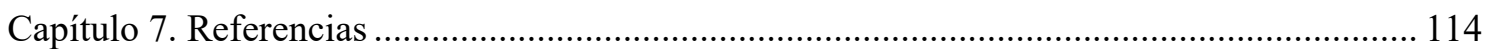

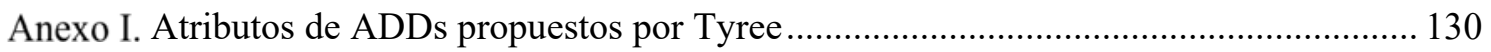

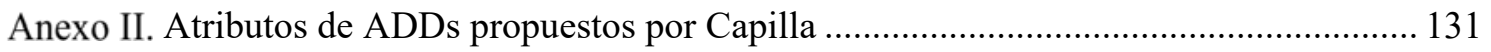

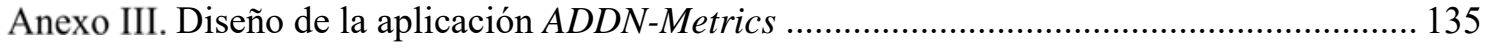

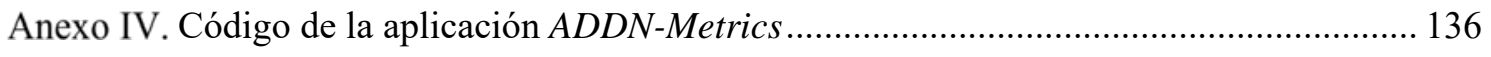

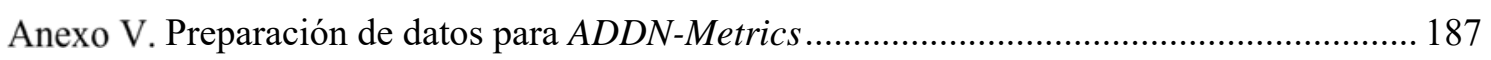

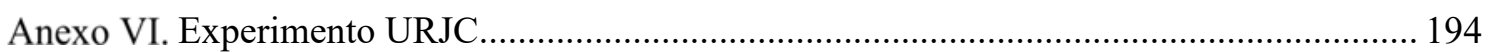




\section{Índice figuras}

Figura 2.1: Meta-modelo ISO/IEC 42010 para describir arquitecturas de sistemas complejos.... 4

Figura 2.2: Ámbito de las decisiones en los espacios del problema y de la solución. .................... 6

Figura 2.3: Modelo conceptual de las decisiones arquitectónicas y su lógica. .............................. 6

Figura 2.4: Modelo de vistas “4+1” con la vista de decisión. .......................................................... 7

Figura 2.5: Modelo para describir decisiones de diseño arquitectónicas. ...................................... 9

Figura 2.6: Meta-modelo para gestionar decisiones de diseño arquitectónicas. ......................... 10

Figura 2.7: Meta-modelo para gestionar decisiones de diseño arquitectónico............................. 11

Figura 2.8: Meta-modelo CoCoADvISE para el modelado de las decisiones de diseño reutilizables

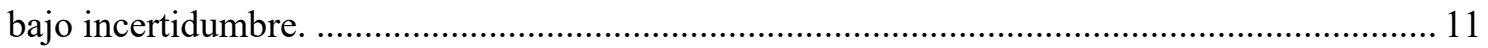

Figura 2.9: Meta-modelo para la gestión del conocimiento....................................................... 12

Figura 2.10: Plantilla para capturar una decisión de diseño con ADDSS 2.0 ............................. 14

Figura 2.11: Presentación de una decisión de diseño con tres alternativas en SAW. .................. 16

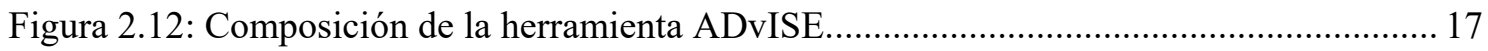

Figura 2.13: Herramienta ADMentor. Diagrama del espacio del problema, navegador del proyecto

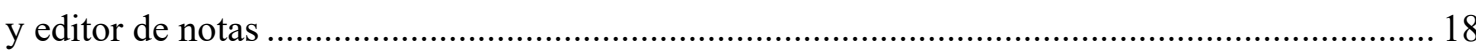

Figura 2.14. Meta-modelo de trazabilidad de la lógica de diseño................................................ 20

Figura 2.15. Estructura de trazabilidad en una descomposición por niveles................................ 20

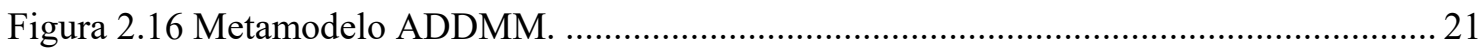

Figura 2.17 Meta-modelo para capturar decisiones de diseño arquitectónico. ............................ 22

Figura 2.18 Modelo para la sostenibilidad de los requisitos de calidad software ....................... 23

Figura 2.19. Conjunto de atributos de calidad para sostenibilidad.............................................. 24

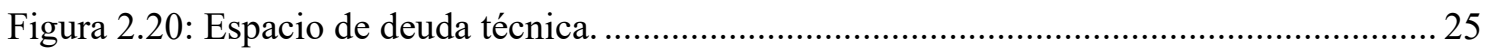

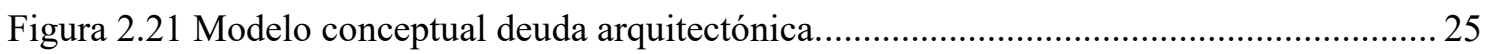

Figura 4.1: Meta-modelo propuesto para la gestión sostenible de decisiones de diseño. ............ 34

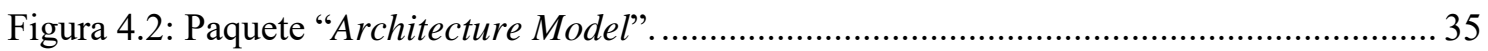

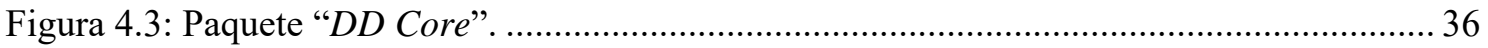

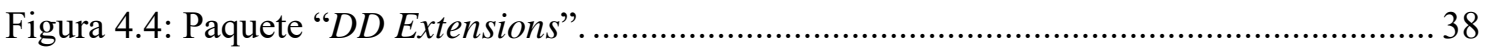

Figura 4.5: Paquete "Sustainability of the Design Decision Model"........................................... 42

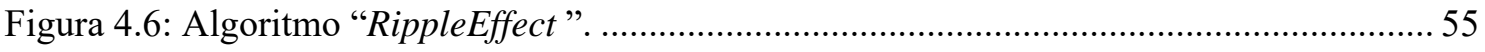

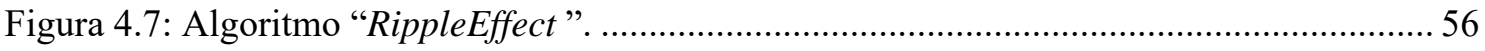

Figura 4.8: Modelo para la gestión sostenible de decisiones de diseño.......................................58

Figura 5.1. Redes de decisiones muy pequeñas a las que se les incluye una decisión aislada.... 61

Figura 5.2. Redes en árbol y con un solo ciclo a las que se les incluye decisiones aisladas....... 61

Figura 5.3. Redes cíclicas con múltiples caminos y sin decisiones aisladas y con ellas............. 62

Figura 5.4. Tres variantes de modelos basados en el modelo Model 7..................................... 63 
Figura 5.5. Tres variantes de modelos basados en el modelo Model 13. 63

Figura 5.6. Estimación de la medida de complejidad para redes muy pequeñas. 64

Figura 5.7. Estimación de la medida de complejidad para redes con árboles y ciclos. 65

Figura 5.8. Estimación de la medida de complejidad para redes que tienen múltiples ciclos.... 65

Figura 5.9. Estimación de la complejidad para las variantes de tipo árbol. ........................... 66

Figura 5.10. Estimación de la complejidad para las variantes de redes cíclicas. .....................66

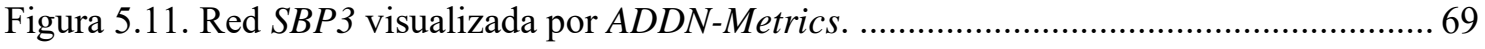

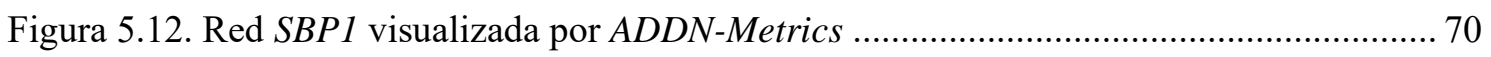

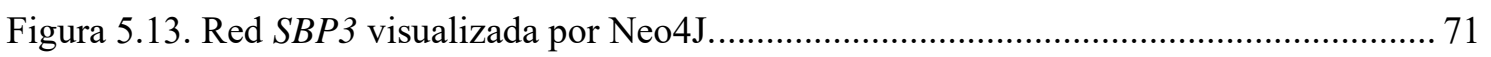

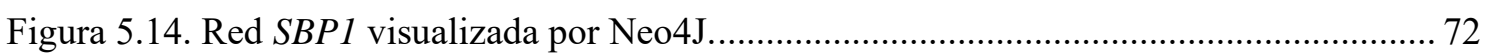

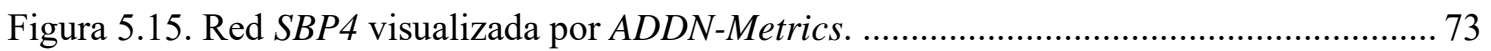

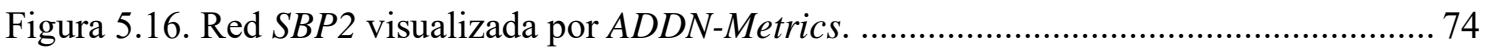

Figura 5.17. Red SBP4 visualizada por Neo4J........................................................ 75

Figura 5.18. Red SBP2 visualizada por Neo4J......................................................... 76

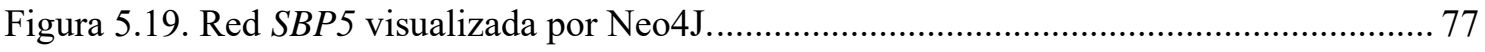

Figura 5.20. Gráfica de complejidad de diferentes topologías sin decisiones aisladas .............. 79

Figura 5.21. Comparativa de complejidad en variantes de redes con topología de árbol. ..........80

Figura 5.22. Evolución de complejidad en variantes de redes con topología con ciclos. ...........81

Figura 5.23. Complejidad en redes Service-based platform sin decisiones aisladas.................. 81

Figura 5.24. Complejidad en redes con decisiones aisladas.............................................. 82

Figura 5.25. Evolución de la complejidad respecto al valor del factor $\alpha$............................83

Figura 5.26. Evolución de la complejidad en redes Service-based platform respecto al factor $\alpha$. .84

Figura 5.27. Tendencia de la complejidad con relación a la variación del factor $\alpha$ en redes Service-

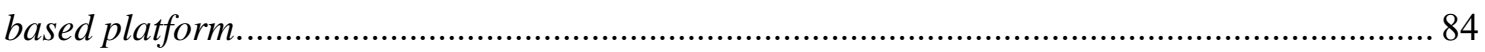

Figura 5.28. Representación gráfica de la decisión de diseño DD01 capturada ...................... 88

Figura 5.29. Esfuerzo realizado en capturar ADDs utilizando diferentes plantillas ................. 90

Figura 5.30. Tiempo (en horas) empleado por cada grupo en capturar alternativas de diseño... 92 Figura 5.31. Relación de tiempo global y tiempo promedio en capturar una decisión y varias alternativas. 93

Figura 5.32. Incremento de esfuerzo utilizando el modelo Mind 1-2 ................................. 96

Figura 5.33. Red SBP6 sin elementos de tipo "question" ........................................... 97

Figura 5.34. Conjuntos de decisiones impactadas en SBP6 por ADD1 ............................... 98

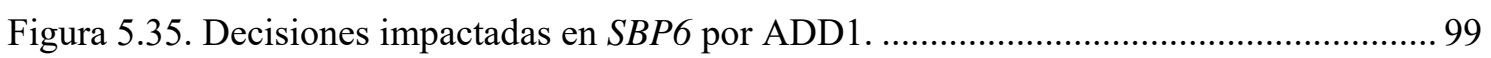

Figura 5.36. Ejemplo de relación entre decisiones..................................................... 100

Figura 5.37. Ejemplo de no relación entre decisiones....................................................... 100

Figura 5.38. Conjuntos de decisiones impactadas en SBP6 por ADD1 en un nivel 2. ............ 101 
Figura 5.39. Valores de instability para las decisiones impactadas por ADD1

Figura 5.40. Conjuntos de decisiones impactadas en SBP6 por ADD3 …................................. 102

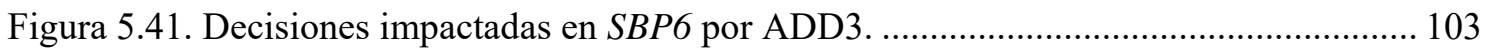

Figura 5.42. Valores de instability para las decisiones impactadas por ADD3 .......................... 104

Figura 5.43. Conjuntos de decisiones impactadas en SBP6 por ADD3 en un nivel 2. ............. 105

Figura 5.44. Conjuntos de decisiones impactadas en SBP6 por ADD3Q1RES ........................ 105

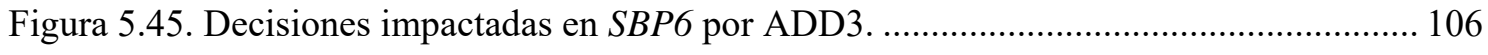

Figura 5.46. Conjuntos de decisiones impactadas en SBP6 por ADD3 en un nivel 2. ............. 107

Figura 5.47. Valores de instability para las decisiones impactadas por ADD3 .......................... 107

Figura 5.48. Conjuntos de decisiones impactadas en SBP7 por WSA_ADD1.......................... 108

Figura 5.49. Valores de instability para las decisiones impactadas por WSA_ADD1............... 108

Figura IV.1: Relación de clases UML de la aplicación $A D D N$-Metrics. ................................... 135

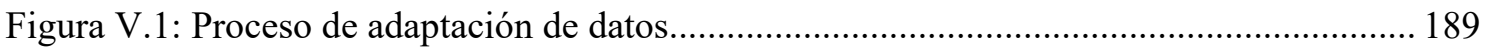




\section{Índice tablas}

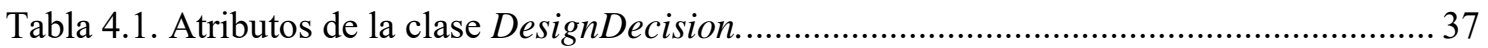

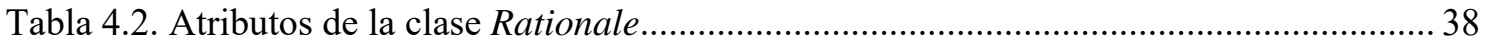

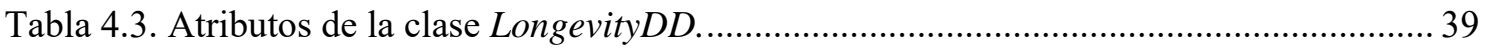

Tabla 4.4. Atributos de la clase ExtendedRationale................................................................... 39

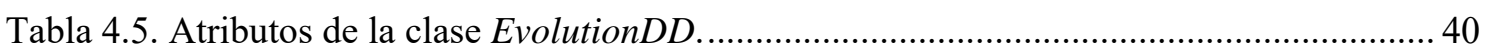

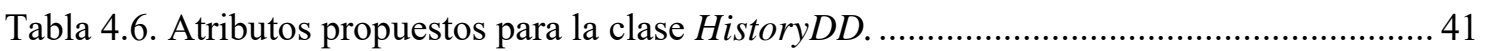

Tabla 4.7 Criterios y métricas para medir la sostenibilidad del conocimiento arquitectónico en al mantenimiento

Tabla 4.8. Criterios y métricas para medir la sostenibilidad del conocimiento arquitectónico en a la evolución. .48

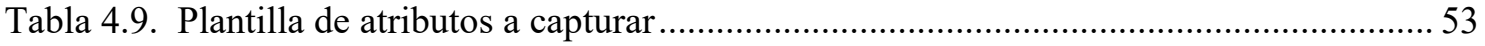

Tabla 4.10. Composición de las plantillas "long", "médium” y "short" .................................... 54

Tabla 5.1. Valores de complejidad obtenidas en redes sin decisiones aisladas............................6 67

Tabla 5.2. Valores de complejidad obtenidas en redes con decisiones aisladas. ........................6 68

Tabla 5.3. Valores de complejidad obtenidas en redes SBP sin decisiones aisladas................... 77

Tabla 5.4. Valores de complejidad obtenidas en redes SBP con decisiones aisladas.................. 78

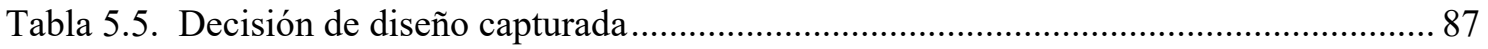

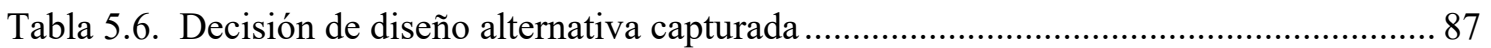

Tabla 5.7. Esfuerzo para capturar decisiones según la plantilla empleada. ................................. 89

Tabla 5.8. Tiempo (en horas) requerido para capturar 1 decisión con múltiples alternativas.... 92

Tabla 5.9. Tiempo (horas) medio estimado para capturar decisiones con múltiples alternativas.

Tabla 5.10. Incremento del esfuerzo (en horas) debido a la actividad de los arquitectos cognitivos, según el modelo Mind1-Mind2.

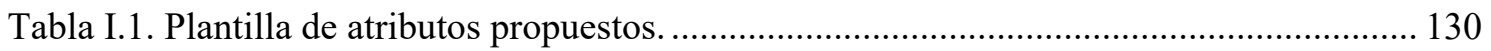

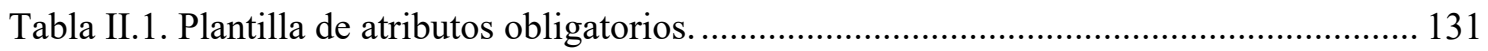

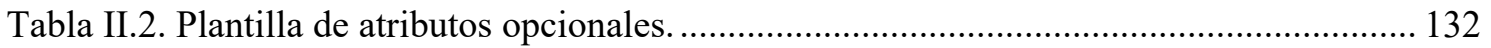

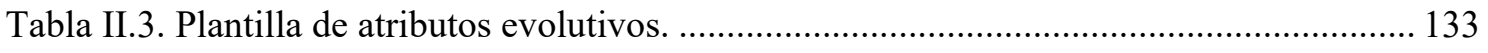

Tabla II.4. Plantilla de atributos evolutivos en las decisiones de diseño. .................................. 133

Tabla II.5. Plantilla de atributos evolutivos en las relaciones entre decisiones.......................... 134

Tabla V.1. Formato del fichero de configuración de una red de decisiones .............................. 187

Tabla V.2. Ejemplo de configuración de una red de decisiones. ............................................... 188

Tabla V.3. Ejemplo de dos decisiones codificadas en formato .json. ......................................... 189 
Tabla V.4. Ejemplo de una relación codificadas en formato .json 190

Tabla V.5. Ejemplo de una relación codificadas en formato .txt 190

Tabla V.6. Ejemplo de un nodo codificadas en formato .CYPHER 190

Tabla V.7. Ejemplo de una relación codificadas en formato CYPHER 191

Tabla V.8. Contenido del fichero de configuración "graphstyle.grass" de Neo4j... 191

Tabla VI.1. Ejemplo de plantilla de atributos de una decisión ya capturada. 196

Tabla VI.2. Datos complementarios basados en la experiencia del arquitecto senior. 197

Tabla VI.3. Datos complementarios basados en la experiencia del arquitecto cognitivo. 198 


\section{Capítulo 1. Introducción}

Actualmente, las Arquitecturas Software constituyen la piedra angular de todo diseño software y no se concibe el desarrollo de ningún sistema de mediana complejidad en el que no exista una arquitectura que le respalde. Además, las arquitecturas software son el nexo entre los requisitos y la implementación y deben ser un fiel reflejo del estado del sistema actual.

El diseño de una arquitectura debe estar dirigido por los requisitos y por parámetros que aseguren su calidad desde las fases tempranas del diseño. Uno de los problemas importantes para medir la calidad en una arquitectura software es evitar la erosión en los diseños y que la arquitectura decaiga. Por ello, con el fin de evitar arquitecturas excesivamente rígidas y decisiones de diseño mal tomadas, se necesita indicadores que nos permitan estimar que partes de las arquitecturas presentan problemas de calidad.

Uno de los aspectos claves en para construir arquitecturas con suficiente calidad es que las decisiones de diseño sean las adecuadas. En base a esto, resulta imprescindible capturar y documentar aquellas decisiones de diseño y su lógica subyacente para poder detectar mejor que partes de la arquitectura presentan problemas de calidad si existen decisiones de diseño mal tomadas o bien decisiones que nunca llegaron a plasmarse en la arquitectura. Entre estas decisiones existe un conocimiento arquitectónico basado en patrones de diseño y otro referente a más prácticas conocido como antipatrones. De esta manera, los antipatrones dan como resultado arquitecturas de baja calidad.

Con el fin de asegurar la calidad de las decisiones tomadas, y que la arquitectura erosione lo menos posible en el tiempo, resulta necesario producir arquitecturas sostenibles y fácilmente mantenible, y por ende que las decisiones de diseño principales sean también sostenibles.

Con el fin de garantizar la calidad de las decisiones de diseño, es necesario contar con métricas adecuadas que midan los diferentes aspectos de calidad relacionados con la sostenibilidad del conjunto de las decisiones tomadas. Mientras que la mayoría de las métricas existentes están enfocadas a código y a medir aspectos de calidad a nivel de 
arquitectura, no existen métricas que midan la calidad y la sostenibilidad de las decisiones de diseño.

En este sentido la falta de modelos y criterios para definir qué se entiende por un conjunto de decisiones sostenible, cual es la cantidad de conocimiento ideal que se precisa capturar y como medir los efectos cuando las decisiones cambian, son aspectos aún no resueltos.

Por estos motivos, el objetivo de este trabajo de Tesis Doctoral es dotar al conocimiento arquitectónico de una gestión más sostenible y para ello propondremos la caracterización de un modelo sostenible que permita el mantenimiento eficiente de las decisiones de diseño en arquitectura software. La introducción de nuevas métricas que permitan medir la sostenibilidad en arquitectura software y en las decisiones de diseño capturadas, resulta clave para estimar y mejorar la calidad de los sistemas. En este trabajo propondremos criterios y métricas para estimar dicha sostenibilidad.

Hemos estructurado esta Tesis Doctoral como sigue. En el capítulo 2 describiremos el estado del arte en Arquitecturas Software centrándonos en aspectos de sostenibilidad en decisiones de diseño. Con las conclusiones obtenidas, en el capítulo 3 presentaremos el problema que queremos resolver dirigido a ofrecer una gestión sostenible del conocimiento arquitectónico. En el capítulo 4 señalaremos las hipótesis de trabajo que delimitaran el ámbito de nuestra solución al problema planteado, solución que será desarrollada en este capítulo. A continuación, en el capítulo 5 validaremos nuestra propuesta de solución. Finalizaremos con el capítulo 6 con las conclusiones y futuras líneas de investigación. 


\section{Capítulo 2. Estado del Arte}

En este capítulo vamos a describir la problemática actual asociada a la representación y gestión de las decisiones de diseño arquitectónicas como elementos de primera clase a la hora de diseñar una arquitectura software. La captura de decisiones de diseño introduce nuevos desafíos, en especial en la obtención de diseños software basados en la gestión sostenible del conocimiento arquitectónico que evoluciona durante la vida del software.

\subsection{Concepto de Arquitectura Software}

La arquitectura software es una disciplina de la Ingeniería Software orientada a describir el diseño de un sistema software. Diferentes autores como Parnas [PARN 89], Perry [PERR 92], Shaw [SHAW 96] y Bass [BASS 98] han sentado las bases para describir el concepto de arquitectura software con el fin de favorecer la modularidad y la reutilización de los diseños software. Las arquitecturas software permiten describir un sistema en términos de componentes y conectores, mejorando los aspectos de comunicación, coordinación y/o cooperación [SHAW 97], así como el comportamiento entre sus diferentes módulos funcionales. Asimismo, la lógica que justifica la selección de un determinado componente se basa en patrones de diseño y estilos arquitectónicos a modo de conocimiento reutilizable para la construcción de arquitecturas software [GAMM 95] [BUSC 96].

Perry et al. [PERR 92] definen el concepto de arquitectura software como "Un conjunto de elementos arquitectónicos que tienen una determinada forma. Las propiedades restringen la elección de los elementos de la arquitectura, mientras que la lógica de diseño captura la motivación de la elección de los elementos y la forma". Para Perry una arquitectura software comprende los componentes (elementos), las propiedades y relaciones entre elementos (forma) y la justificación (lógica) de las decisiones que se han tomado para llegar a esa arquitectura guiada por sus atributos de calidad. De esta manera, podemos observar que los autores dan importancia a la lógica en la toma de decisiones de diseño software. 
Las definiciones contemporáneas de arquitectura software consideran a ésta como la estructura o estructuras de un sistema que comprende componentes, las propiedades visibles externas de dichos componentes y las relaciones entre ellos [BASS 98] [BASS 13], y sugieren que una arquitectura software debe reflejar las decisiones de diseño tempranas que son las que más influyen en las etapas iniciales del desarrollo software. Por ello, los autores en [VGUR 02] resaltan la importancia de las decisiones para evitar que se evapore este conocimiento arquitectónico. Kruchten [KRUC 04] y Bosch [BOSC 04] señalan la importancia de las decisiones de diseño al considerarlas como elementos de primera clase para describir una arquitectura software.

En este sentido, las diferentes propuestas sobre decisiones de diseño en arquitectura software, que detallaremos posteriormente, han dado lugar al reciente estándar ISO/IEC 42010 [ISO 42010:11] el cual define las decisiones de diseño y su lógica ("Architecture Rationale") como elementos de primera clase tal y como muestra la figura 2.1. Los elementos arquitectónicos que representan un sistema mediante diferentes vistas arquitectónicas tienen su reflejo en un conjunto de decisiones de diseño y la lógica que motivan la elección de los mismos.

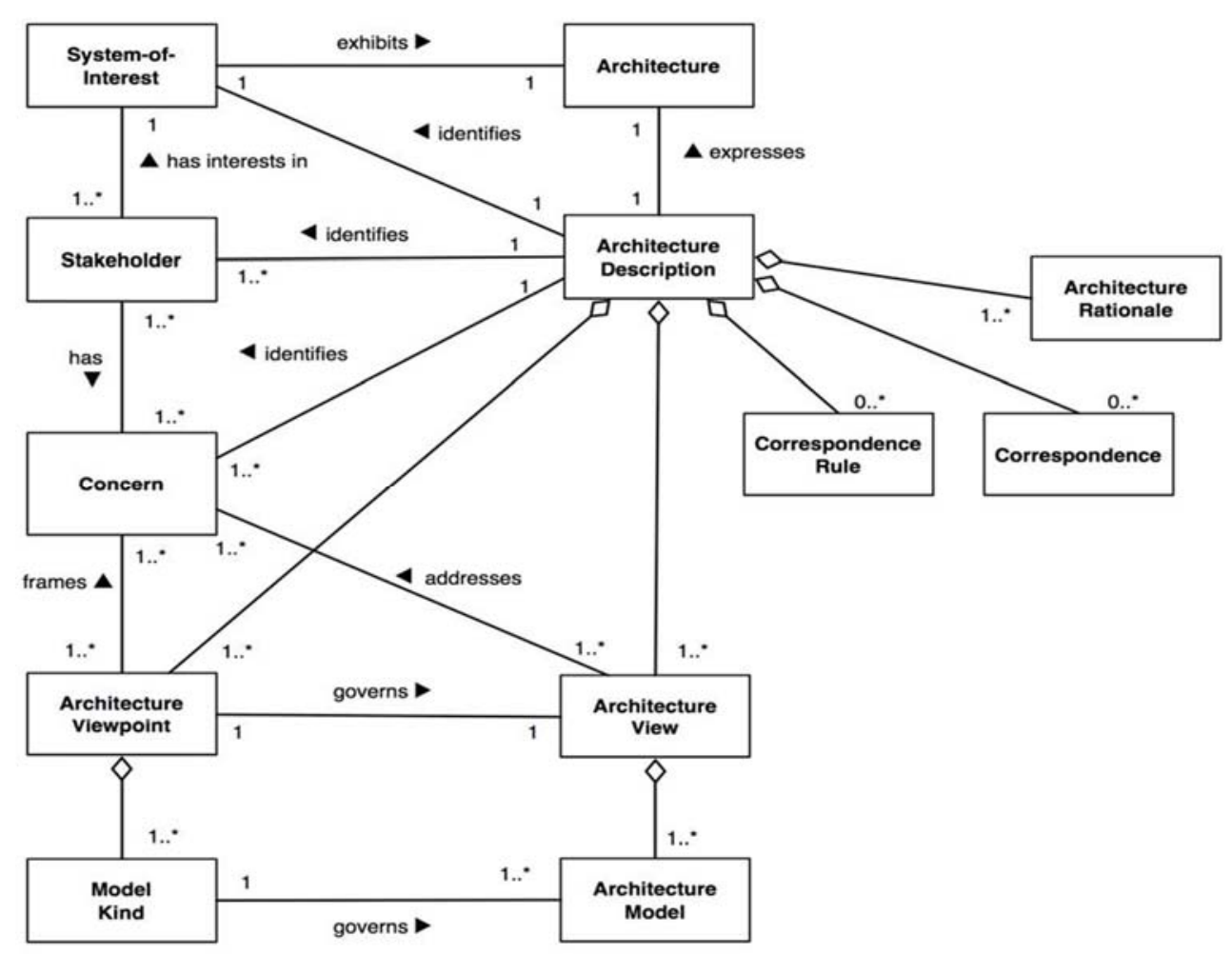

Figura 2.1: Meta-modelo ISO/IEC 42010 para describir arquitecturas de sistemas complejos. 


\subsection{Decisiones de Diseño en Arquitectura Software}

Debido a la importancia de las decisiones de diseño en arquitectura software, vamos a describir en esta sección el concepto de decisión de diseño y como se relaciona con la arquitectura software. Una de las primeras definiciones sobre decisión de diseño arquitectónica es presentada por Jansen y Bosch [JANS 04], [BOSC 04] como:

"una descripción del conjunto de adiciones, eliminaciones y modificaciones a la arquitectura software, el fundamento, las reglas y restricciones de diseño y los requisitos adicionales que dan respuesta a uno o más requisitos en una arquitectura dada”.

Con esta definición, los autores señalan que una decisión afecta a la arquitectura con sus reglas y restricciones de diseño para cumplir los requisitos y que el fundamento o lógica justifica la elección de la decisión seleccionada.

Las decisiones de diseño constituyen un conocimiento tácito que es difícil de formalizar. Por ello, Kruchten [KRUC 04] distingue entre decisiones explicitas e implícitas, y que algunas de estas últimas se diluyen a lo largo del proceso de diseño, llegando a desaparecer completamente debido a que no están documentadas. El autor sugiere organizar el conocimiento en el diseño arquitectónico mediante decisiones de diseño y sus atributos, además de las relaciones de dichas decisiones con otros productos arquitectónicos a lo largo del ciclo de vida.

Otros autores [VVEN 06] [BOER 07] describen que una decisión de diseño es aquella que se elige entre un conjunto de decisiones alternativas y en esta elección se aplica la lógica, las reglas y las limitaciones de diseño. Por otra parte, cuando no se capturan y documentan las decisiones de diseño se evapora parte del conocimiento arquitectónico, con el fin de evitar la erosión en la arquitectura. Otros autores [JANS 08B] expresan la necesidad de documentar la arquitectura software durante el proceso de toma de decisiones.

Según Lago et al. [LAGO 06] las decisiones de diseño se toman en el ámbito de la solución del diseño arquitectónico (figura 2.2). Los autores argumentan que las decisiones relacionadas con los requisitos se toman en el denominado ámbito de problema y que existe un conjunto de decisiones arquitectónicas que se toman en ambos espacios, pero cuyos efectos se reflejan en el espacio de la solución. 


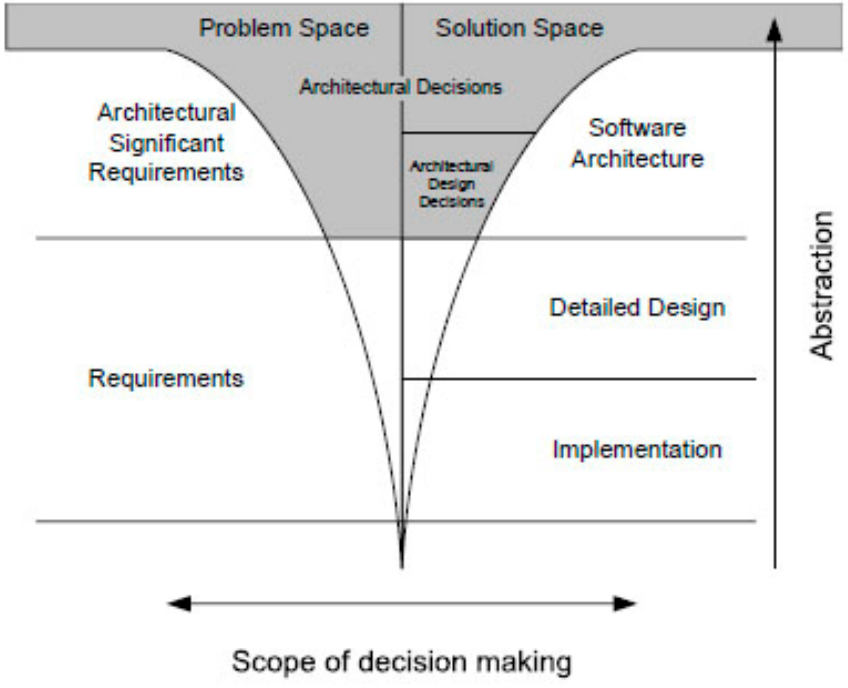

Figura 2.2: Ámbito de las decisiones en los espacios del problema y de la solución.

El concepto básico de decisión de diseño se ha plasmado en el estándar ISO/IEC 42010 [ISO 42010:11], que establece un modelo conceptual para decisiones de diseño arquitectónicas. Este estándar considera a las decisiones arquitectónicas (Architecture Decision) como elementos de primera clase y que tratan de resolver problemas de diseño (Concern). Estas decisiones de diseño dan como resultado la descripción de elementos en la arquitectura software ( $A D$ Element) y que se justifican con una argumentación apropiada (Architecture Rationale) que describen los "pros" y los "cons" de cada decisión. Este modelo conceptual descrito se muestra en la figura 2.3.

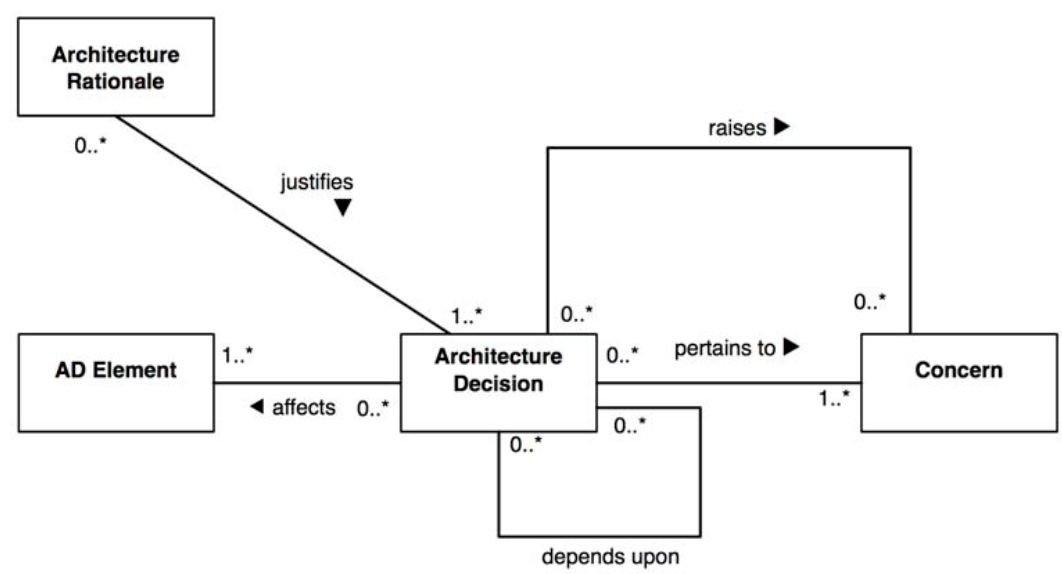

Figura 2.3: Modelo conceptual de las decisiones arquitectónicas y su lógica.

Una vez presentado el término decisión de diseño, vamos a analizar cómo representar las decisiones de diseño utilizando el concepto de vista arquitectónica indicado en el estándar ISO/IEC 42010. Posteriormente analizaremos algunas propuestas de 
caracterización de las decisiones de diseño y los meta-modelos más importantes que soportan la caracterización de las decisiones de diseño en la arquitectura.

Vista de la decisión: Diversos autores [KRUC 95], [SONI 95], [CLEM 03] y [ROZA 05] proponen diferentes modelos de vistas arquitectónicas para describir una arquitectura software. La necesidad de documentar las decisiones de diseño como entidades de primera clase ha llevado a algunos autores a considerar una nueva vista arquitectónica para representar este conocimiento. Dueñas et al. [DUEÑ 05] proponen la "vista de la decisión" porque consideran que la representación de las decisiones difiere a la del resto de las vistas. Esta vista permite documentar aspectos importantes como las relaciones entre decisiones y requisitos y las arquitecturas como medio para cubrir el tradicional hueco existente entre requisitos y diseño. Así, las decisiones de diseño, que pertenecen a la vista de la decisión, permiten conectar los requisitos con la información de las tradicionales vistas arquitectónicas del modelo de “4+1”. Kruchten et al. [KRUC 09] describe una nueva vista denominada "vista de la decisión" para reflejar la importancia de las decisiones en la arquitectura y sus vistas, tal y como se muestra en la figura 2.4 .

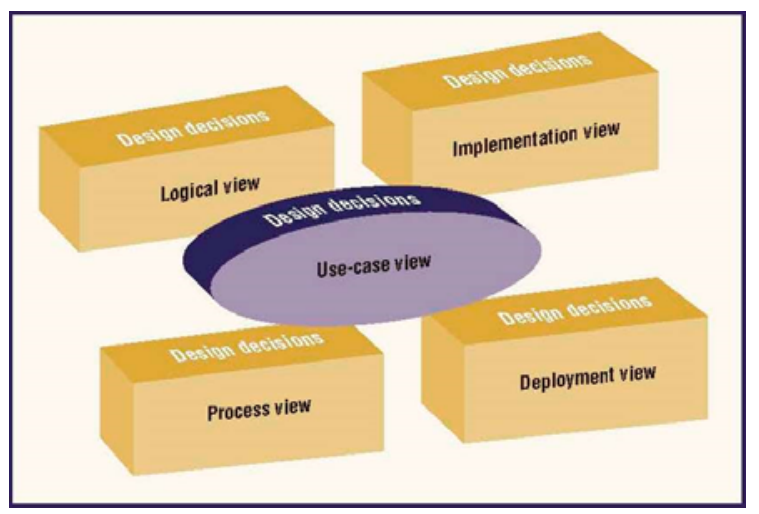

Figura 2.4: Modelo de vistas " $4+1$ " con la vista de decisión.

\subsubsection{Caracterización de decisiones de diseño}

Con el fin de capturar decisiones de diseño es necesario determinar cómo caracterizar el conocimiento. En este sentido, Lee et al. [LEE 04] señalan que es necesario proporcionar una representación adecuada de las decisiones, con el objetivo de determinar los atributos más relevantes para poder documentar la lógica de las decisiones. 
Una de las primeras propuestas de caracterización fue presentada por Tyree et al. [TYRE 05]. Los autores exponen que, en los procesos de desarrollo arquitectónico, la continua toma de decisiones no se documenta de forma explícita y que las decisiones estructurales no quedan reflejadas en las tradicionales vistas arquitectónicas. En este sentido, los autores hacen hincapié en reflejar en una documentación ágil y actualizada (i) los motivos de las decisiones de diseño tomadas, (ii) la lógica que las motivó y las alternativas consideradas, (iii) los cambios ocasionados por cada elección y sus implicaciones en la arquitectura. Además, los autores proponen una plantilla de atributos para caracterizar y capturar el conocimiento de las decisiones de diseño (Anexo I, tabla I.1) pero no los procesos ni el soporte de herramientas para llevar a cabo estas tareas.

Capilla et al. [CAPI 07A] proponen que la captura y descripción de las decisiones debe ser flexible. Por ello, y a diferencia de la propuesta de Tyree, los autores clasifican los atributos a capturar en obligatorios (Anexo II, tabla II.1) y opcionales (Anexo II, tabla II.2). Los autores también establecen que algunos atributos son más útiles durante la evolución de las decisiones (Anexo II, tabla II.3) y que algunos de ellos deberían ser registrados cuando la arquitectura es diseñada por primera vez. Zimmermann [ZIMM 11] relaciona los atributos de calidad con el concepto de decisiones arquitectónicas. Para el autor, las decisiones capturan los aspectos importantes de diseño y justifican la solución elegida en base a estos atributos y resaltando la importancia de documentar las mismas.

\subsubsection{Meta-modelos para la representación de decisiones de diseño}

En esta sección analizaremos algunos de los más importantes meta-modelos propuestos orientados a la representación y captura de decisiones de diseño. Uno de los primeros meta-modelos para la captura de las decisiones de diseño analizados es el propuesto por Jansen et al [JANS 05] (figura 2.5) y es utilizado en la herramienta Archium. Los autores se centran en las decisiones de diseño, tomadas en un contexto determinado, y en cómo estas dirigen una solución particular a un problema que pretenden resolver, donde cada solución particular tendrá sus propias reglas de diseño, restricciones, consecuencias y los pros y contras asociados de tomar esa decisión. El modelo presenta las relaciones entre decisión y la lógica que la motivó, y entre decisión y los cambios arquitectónicos explícitos que produce. 


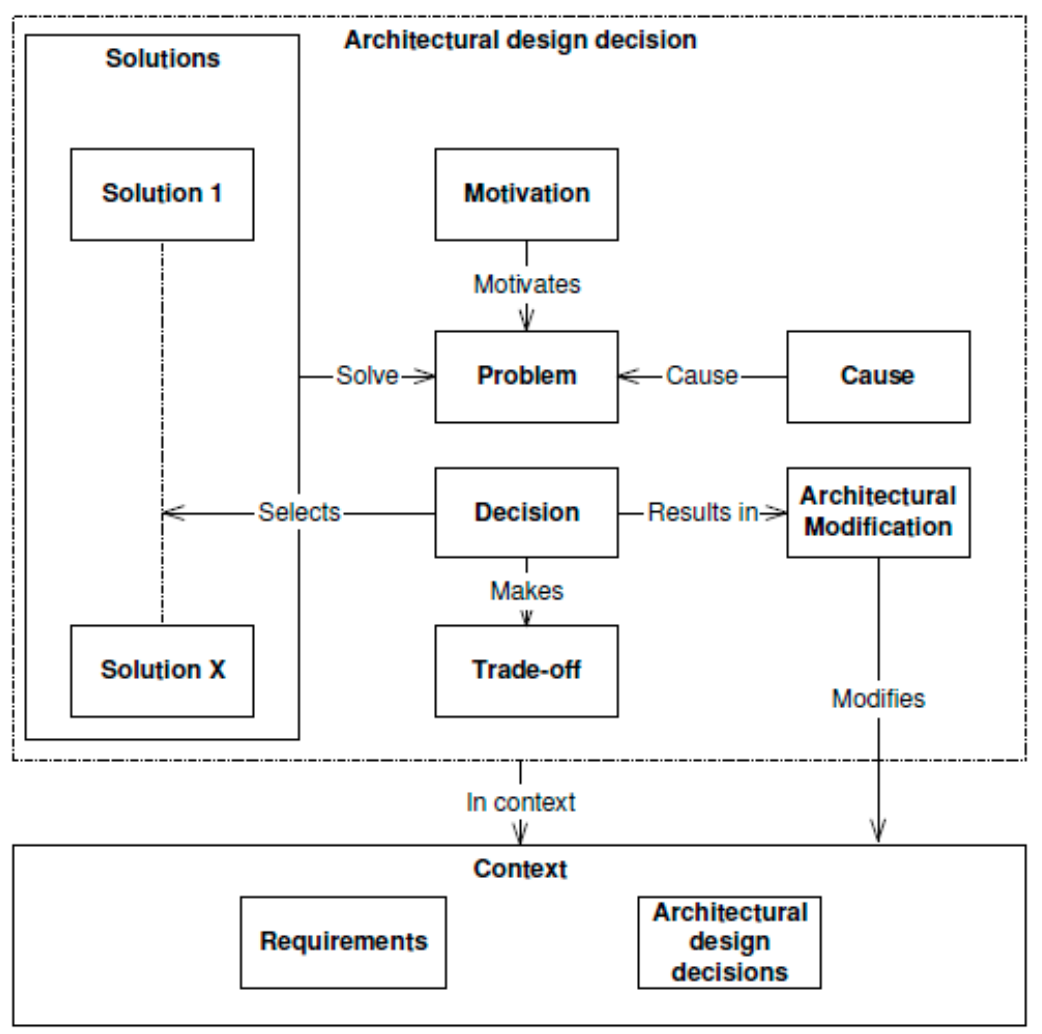

Figura 2.5: Modelo para describir decisiones de diseño arquitectónicas.

A pesar que Jansen presenta un meta-modelo para modelar arquitecturas software centrado en las decisiones de diseño, los autores no determinan como clasificar las decisiones de diseño, sus atributos y las relaciones existentes entre estas y el resto de productos software.

Otros de los meta-modelos que analizamos es el propuesto por Capilla et al. [CAPI 07A], donde las decisiones de diseño enlazan los requisitos y los productos arquitectónicos para registrar el fundamento de las decisiones tomadas durante el proceso de construcción del diseño y sus relaciones (figura 2.6). El meta-modelo está formado por los siguientes elementos: (i) Modelo del proyecto orientado a organizar la arquitectura en sucesivas iteraciones, así como las decisiones tomadas, (ii) Arquitectura donde se representan las soluciones de diseño, (iii) Modelo de la decisión donde se representan las decisiones de diseño capturadas incluyendo la lógica de las mismas. 


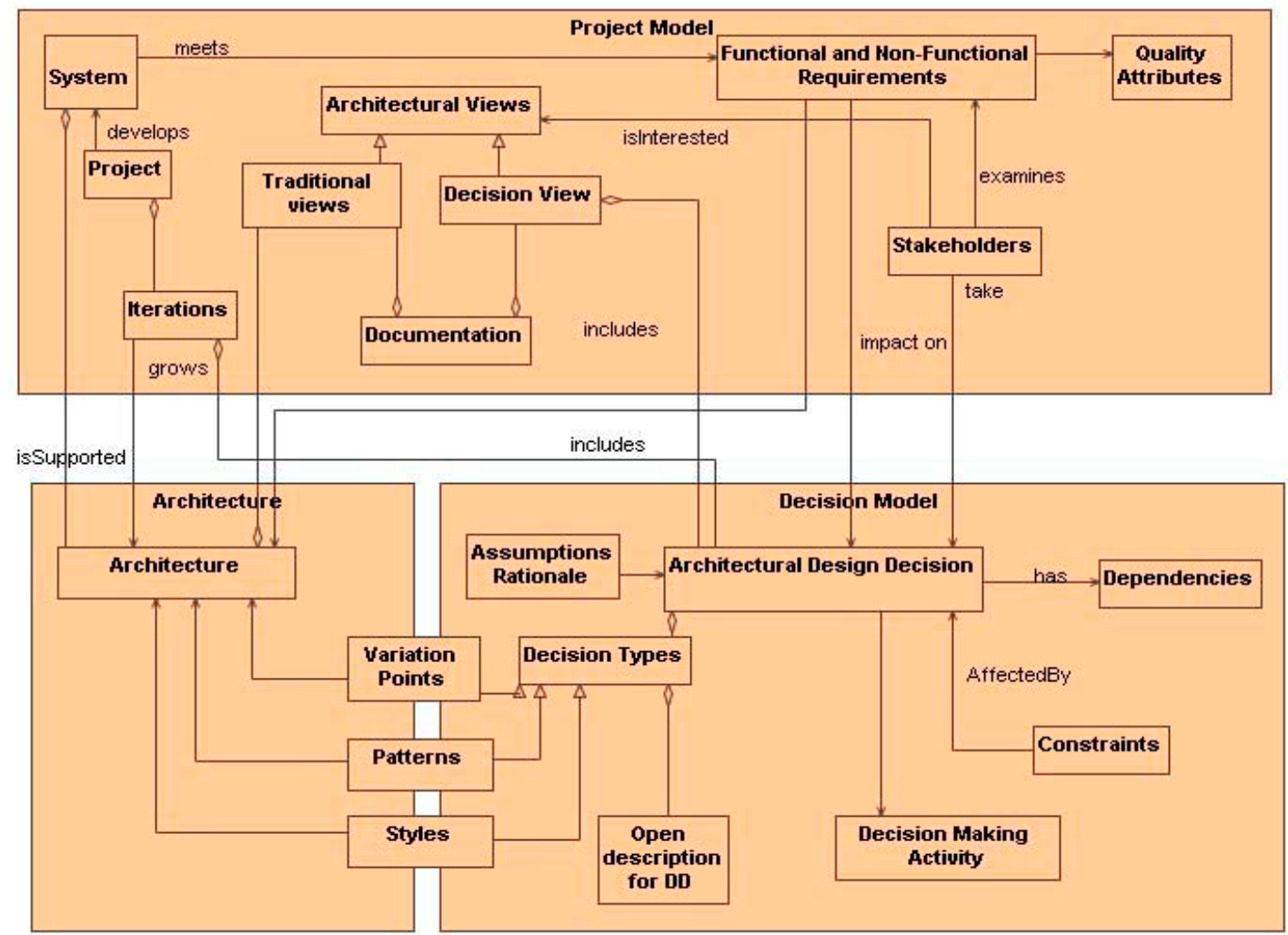

Figura 2.6: Meta-modelo para gestionar decisiones de diseño arquitectónicas.

El modelo de decisión de diseño está preparado para gestionar el conocimiento teniendo en cuenta los atributos obligatorios (Anexo II, tabla II.1) y opcionales (Anexo II, tabla II.2) de las decisiones.

Otro de los meta-modelos para representar decisiones de diseño es el propuesto por Zimmermann et al. [ZIMM 07], el cual organiza las decisiones de diseño alrededor de una jerarquía u organización de diseño (ADTopic) tal y como se muestra en la figura 2.7. Este modelo define un proceso de tres fases para tomar decisiones de diseño: (i) identificar la decisión en cada proyecto de desarrollo, (ii) elección de la decisión guiada por los requisitos o por los atributos de calidad del software seleccionado alternativas, y (iii) ejecutar la decisión analizando y compartiendo sus efectos en toda la arquitectura. Las decisiones de diseño elegidas y sus alternativas se capturan en las clases ArchitecturalDecision y ADAlternative respectivamente. El resultado de tomar cada decisión es capturado en ADOutcome, incluyendo la justificación y que elementos afecta. 


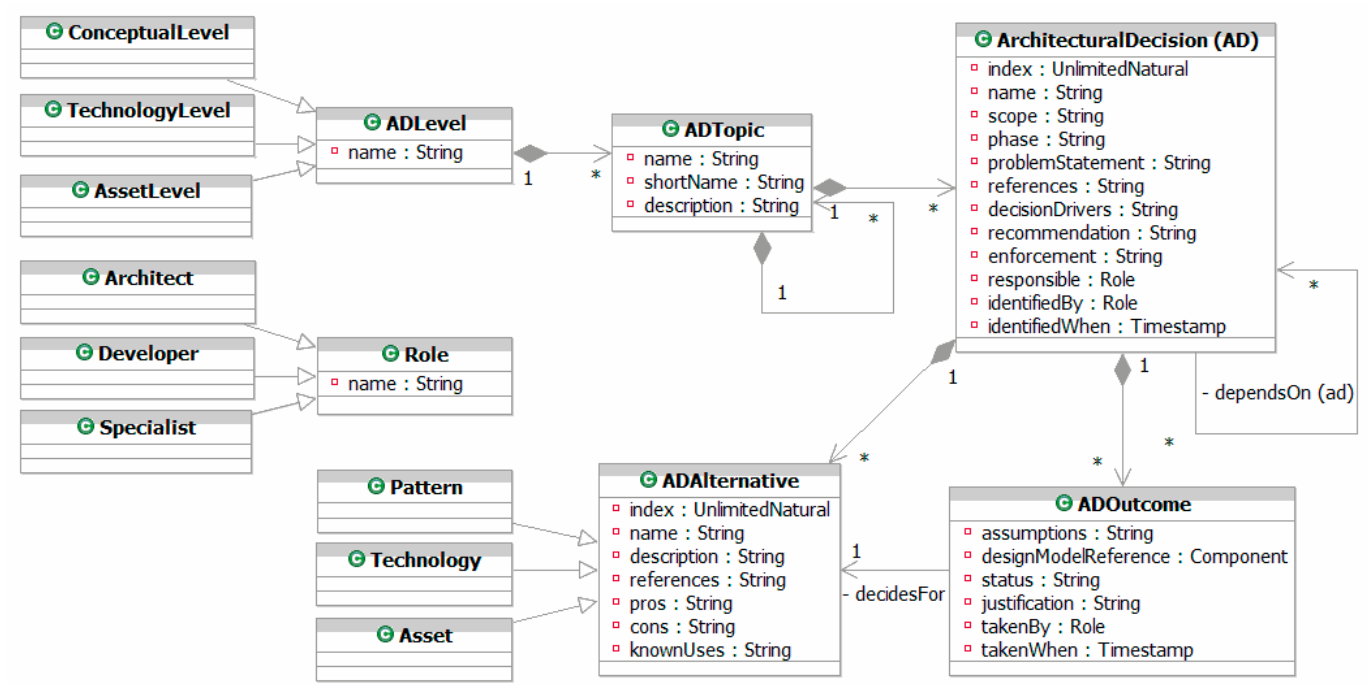

Figura 2.7: Meta-modelo para gestionar decisiones de diseño arquitectónico.

Por otra parte, el meta-modelo CoCoADvISE [STEV 16] integra tácticas para la toma de decisiones de diseño reutilizables bajo incertidumbre guiadas por atributos de calidad (figura 2.8), Estas tácticas son decisiones de diseño que persiguen mejorar los atributos de calidad. El meta-modelo propuesto por Stevantic, desarrollado a partir de [LYTR 15A] [LYTR 13], se basa en el método QOC (Question, Options and Criteria), que utiliza un conjunto de preguntas (Question) y opciones (Option) que posibilitan la toma de decisiones (Decision). Las soluciones seleccionadas, junto a las guías de diseño y guías arquitectónicas, impactan en los atributos de calidad (Quality Attribute).

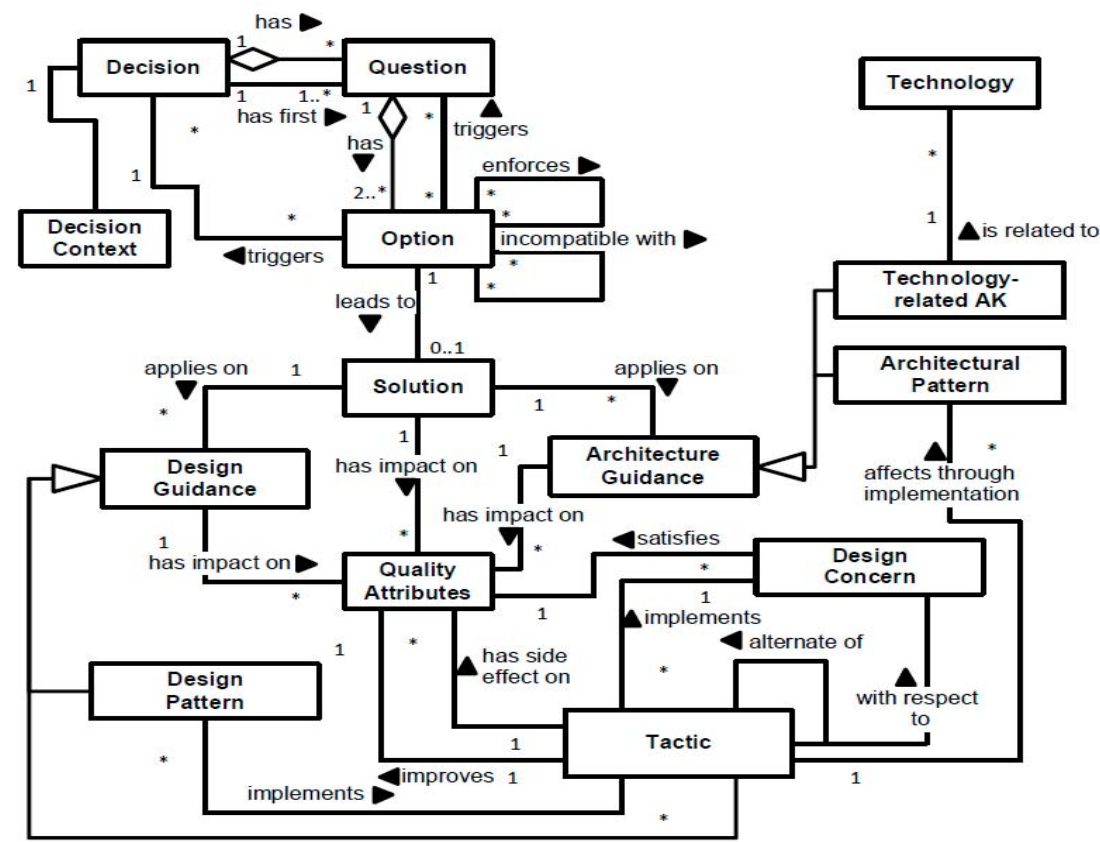

Figura 2.8: Meta-modelo CoCoADvISE para el modelado de las decisiones de diseño reutilizables bajo incertidumbre. 
Finalmente, algunas experiencias recientes como la que se describe en [BHAT 16], proponen un metamodelo (figura 2.9) para la gestión del conocimiento arquitectónico que se aplica en un contexto industrial, referido a la captura y gestión de decisiones de diseño colaborativos en diferentes dominios de negocio. Sin embargo, el metamodelo propuesto no aporta ninguna novedad conceptual respecto a los metamodelos anteriores.

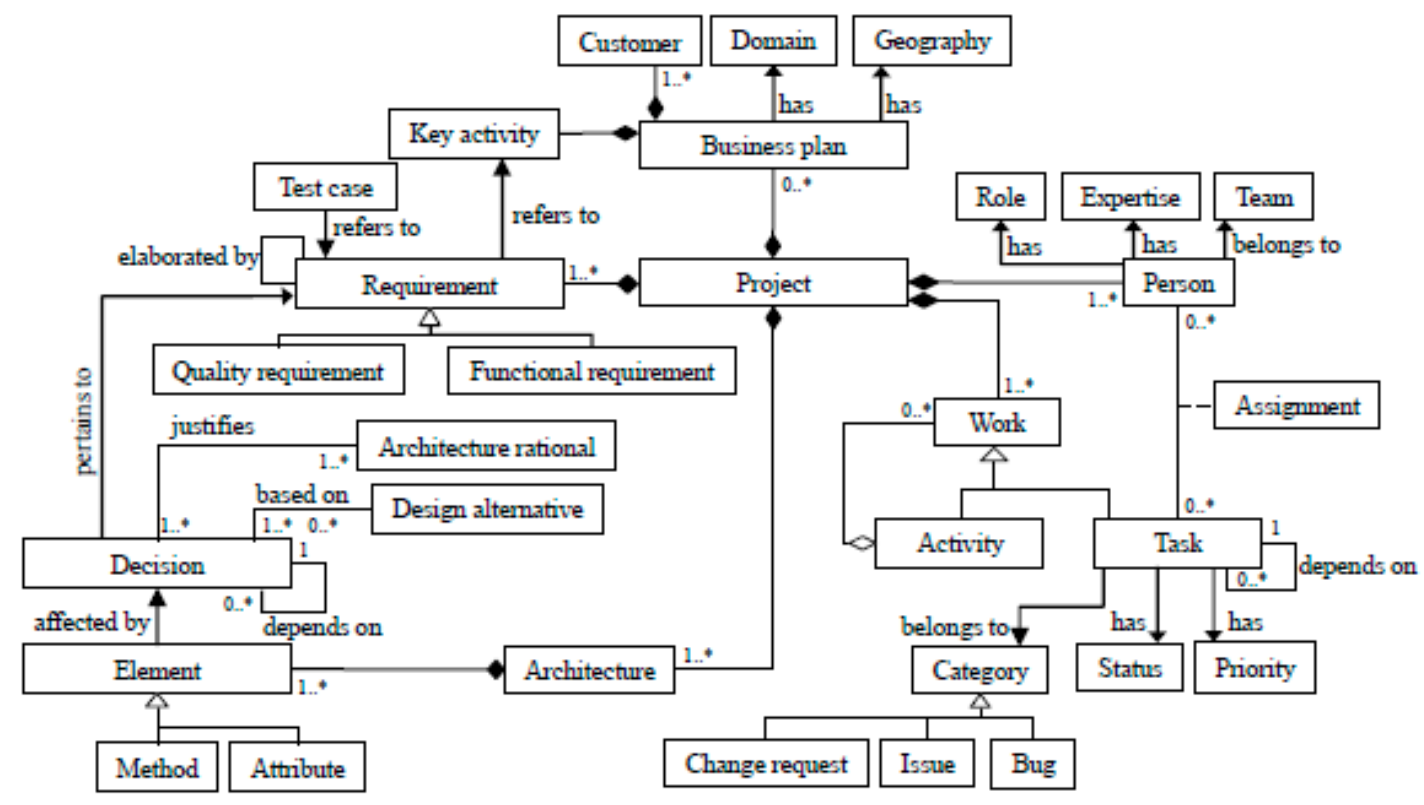

Figura 2.9: Meta-modelo para la gestión del conocimiento.

\subsection{Herramientas para la Captura de Decisiones de Diseño}

Debido a que la mayoría de los meta-modelos presentados se han utilizado para construir herramientas que permiten la captura y gestión de las decisiones de diseño, en esta sección describimos algunas de las herramientas más relevantes organizadas en tres generaciones o periodos diferentes, tal y como se refleja en [CAPI 16].

\subsubsection{Periodo 2004-2006}

Las herramientas que se crearon en este periodo se centraron principalmente en capturar y gestionar el conocimiento arquitectónico. Casi todas ellas se basan en las plantillas para la captura de atributos de Tyree et al. [TYRE 05]. Algunas de las herramientas más importantes en este periodo son las siguientes. 
Archium es una herramienta Java [JANS 07] que utiliza un lenguaje de descripción de arquitectura $(A D L)$ integrado en Java para representar y visualizar las decisiones mediante un grafo de dependencias. Además, permite comprobar la consistencia de las decisiones y potenciales violaciones de diseño. Archium soporta trazabilidad tanto en las relaciones formales, semánticamente correctas, como en las informales, donde se establece un enlace entre la descripción textual y los elementos del modelo exceptuando los requisitos de calidad. Esta herramienta se basa en el meta-modelo del mismo nombre descrito en nuestra sección 2.2.4.

PAKME (Process-based Architecture Knowledge Management Environment) [BABA 05], [BABA 07] es una herramienta Web orientada a la captura, gestión y documentación del conocimiento arquitectónico, y que está basada en la plataforma abierta Hipergate [@HIPE]. PAKME ofrece colaboración en línea y utiliza un conjunto de plantillas para capturar el conocimiento de las decisiones de diseño y los escenarios que las motivan. Además, permite capturar patrones para su reutilización posterior y opciones de diseño con la descripción de la lógica para cada opción. PAKME permite relacionar decisiones con atributos de calidad y escenarios con el fin de tomar decisiones basada en atributos de calidad.

AREL (Architecture Rationale and Elements Linkage) captura decisiones de diseño y modela las relaciones entre decisiones [TANG 05]. También incluye la lógica de diseño tanto cualitativa porque incluye la argumentación a favor y en contra de una alternativa de diseño, como cuantitativamente al utilizar costes, beneficios y riesgos para estimar los méritos de dicha alternativa. Además, soporta la evolución de la arquitectura al incorporar un histórico de decisiones y los motivos de dichos cambios, a través de la extensión eAREL [TANG 07], e incorpora técnicas automáticas para visualizar la trazabilidad bidireccional. Las relaciones "hacia adelante" permiten analizar el impacto de las decisiones en la arquitectura y las relaciones "hacia atrás" identifican el origen de cambios en la arquitectura o incluso el sistema. También permite trazabilidad selectiva basada en la clasificación de elementos arquitectónicos, reduciendo la sobrecarga de información en el análisis de los resultados. Una de las principales ventajas de AREL, frente a otras herramientas, es que las decisiones se capturan al tiempo que se modela la arquitectura, de manera que solamente es necesaria una única herramienta. 
ADDSS (Architecture Design Decision Support System) es una herramienta Web para capturar, gestionar y documentar decisiones de diseño, proporcionando trazabilidad entre los requisitos, decisiones y modelos arquitectónicos que se capturan en sucesivas iteraciones [CAPI 06]. La herramienta permite describir el estado de las decisiones y su evolución, así como reutilizar patrones de diseño. ADDSS captura distintos tipos de decisiones incluyendo las alternativas y la lógica de las mismas y genera de manera automática la documentación que incluye a dichas decisiones. Sin embargo, los modelos arquitectónicos deben generarse con una herramienta externa que posteriormente son incorporados por ADDSS en cada iteración del proceso de construcción de la arquitectura. La figura 2.10 muestra una captura de ADDSS en el que requisitos software se asocian a decisiones de diseño.

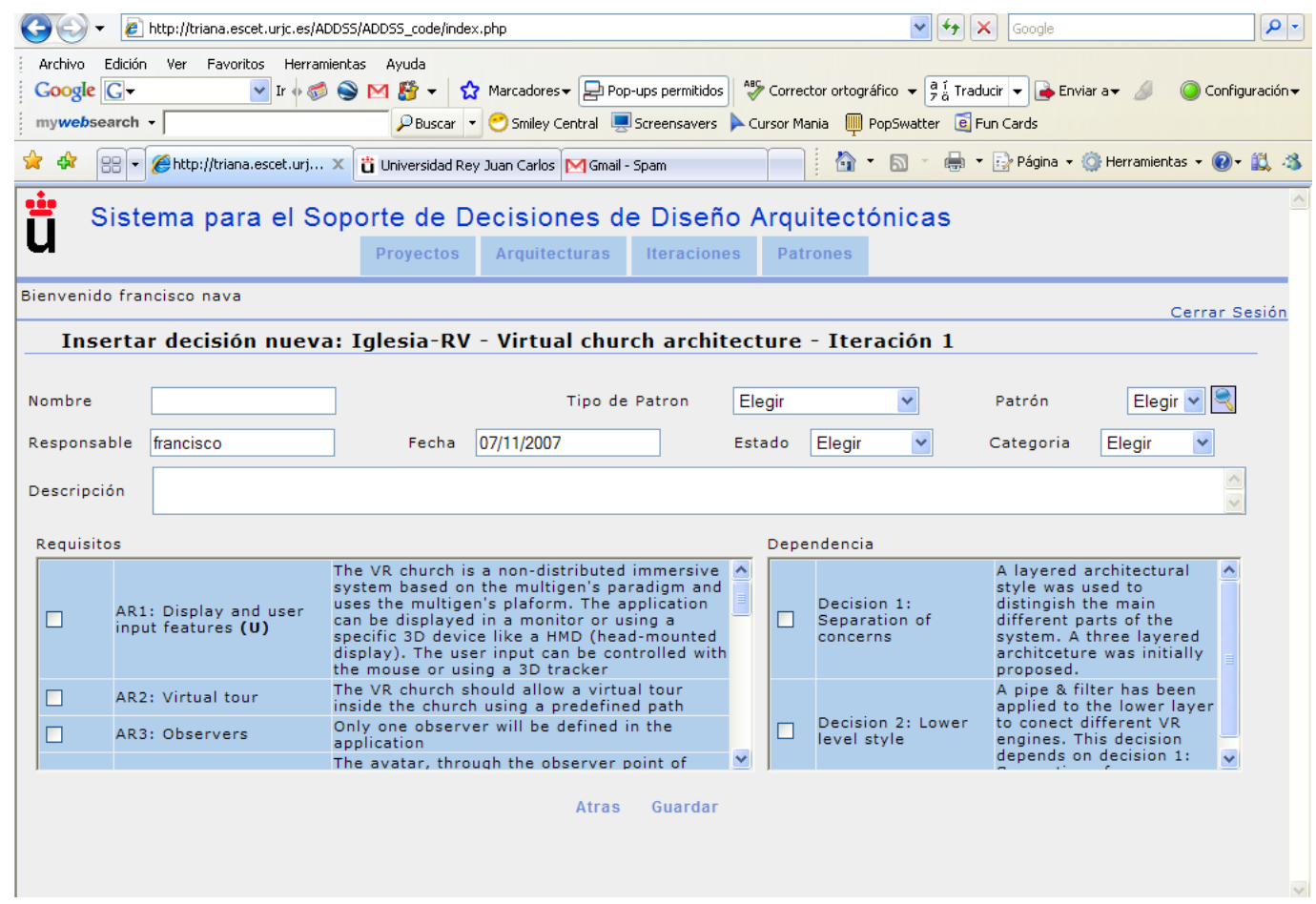

Figura 2.10: Plantilla para capturar una decisión de diseño con ADDSS 2.0.

Con ADDSS 2.0, la captura del conocimiento arquitectónico y su visualización es iterativa. Además de documentar el conocimiento de manera automática, la herramienta permite relacionar bidireccionalmente decisiones entre sí, y estas con requisitos y arquitecturas. Con estas relaciones se puede estimar el impacto debido a cualquier cambio arquitectónico. Sin embargo, la herramienta no soporta trazabilidad de grano fino entre las decisiones y los elementos de una arquitectura concreta [CAPI 11]. 


\subsubsection{Periodo 2007-2010}

Durante este periodo las herramientas para capturar decisiones de diseño se han centrado mayormente en la compartición del conocimiento arquitectónico, tal y como explicamos a continuación. La herramienta EAGLE (Environment for Architects to Gain and Leverage Expertise) [FARE 07] es una aplicación Web desarrollada para compartir el conocimiento arquitectónico que está codificado en un repositorio. La técnica de compartición es múltiple al incorporar Wikis, blogs, archivos RSS (Really Simple Syndication) e incluso técnicas de minería de datos para la extracción de información a compartir.

The Knowledge Architect es una suite de herramientas para capturar, gestionar y compartir el conocimiento de las arquitecturas [JANS 08A]. Utiliza un modelo clienteservidor para almacenar entidades de conocimiento a las que acceden clientes Word que son los encargados de capturar las decisiones de diseño. Permite enlazar decisiones de diseño con arquitecturas, que se modelan con herramientas externas y que se incrustan en los documentos MS-Word. Esta herramienta difiere de las anteriores en que no captura la información de las decisiones mediante plantillas estructuradas, sino que utiliza el propio texto Word de las decisiones para capturarlas. La herramienta permite la exploración de conocimiento mediante un plug-in de navegación Web y enlaces entre las entidades de conocimiento, refinando los atributos de calidad abstractos en otros más concretos. Además, la herramienta ofrece un control de versiones para trazar la evolución de cada entidad del conocimiento [CAPI 11].

ADWiki (Architectural Decision Knowledge Wiki) [SCHU 07] se basa en tecnología Web 2.0 para soportar la toma de decisiones de manera cooperativa. ADWiki está construido a partir de un software wiki utilizando para ello el meta-modelo planteado por Zimmermann et al. [ZIMM 07]. La herramienta ofrece (i) una gestión completa de proyectos, decisiones y alternativas, (ii) una gestión de dependencias de decisiones, (iii) un editor de relaciones entre decisiones (iv) un gestor de ciclo de vida de las decisiones incluyendo el ámbito, las reglas y el estado, (v) identificación de las decisiones a través de su interfaz de usuario y (vi) facilita la integración de la evolución temporal y de las decisiones en tiempo real. 


\subsubsection{Periodo 2011-2017}

En este último periodo las herramientas gestión de conocimiento arquitectónico se han centrado en capacidades más avanzadas como son la reutilización de conocimiento y capacidades colaborativas que extienden la compartición del conocimiento.

Nowak et al. [NOWA 13] presentan la herramienta Web SAW (Software Architecture Warehouse) que permite tomar y compartir decisiones de diseño de manera colaborativa y distribuida. Cada miembro del equipo aporta su opinión motivada sobre cada decisión que, al ser difundida al equipo, es analizada y votada. Como consecuencia del mecanismo de compartición utilizado, SAW permite detectar decisiones alternativas no aceptadas o no rechazadas, decisiones con valoraciones conflictivas o decisiones con múltiples alternativas que deberían ser re-evaluadas. En la figura 2.11 se observa como la información relacionada con una decisión de diseño se comparte entre los usuarios, que votan sobre las diferentes alternativas en base a la lógica descrita para cada decisión.

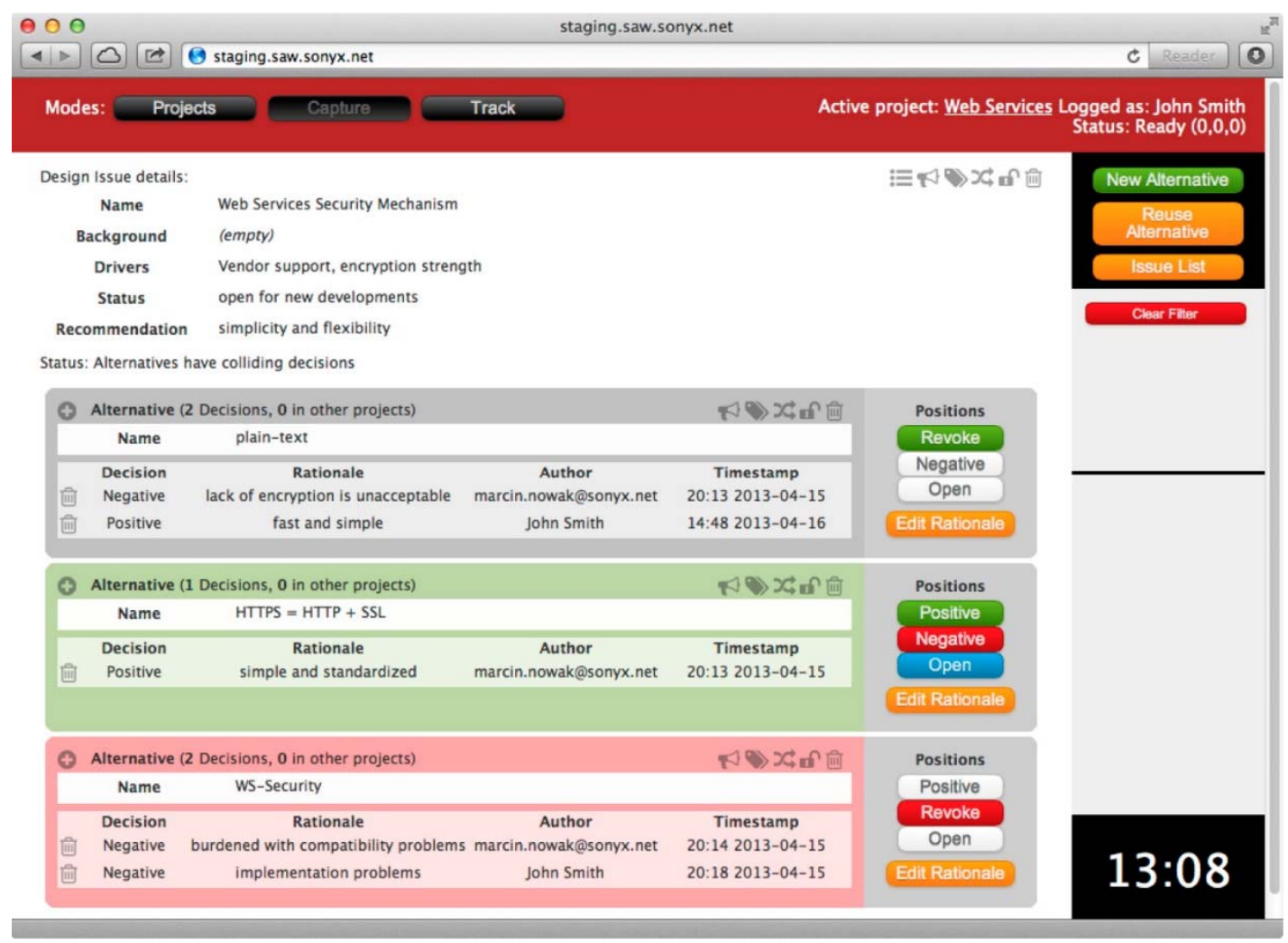

Figura 2.11: Presentación de una decisión de diseño con tres alternativas en SAW. 
ADvISE (Architectural Design decIsion Support framEwork) [@ADvISE] está basado en el meta-modelo presentado por Lytra et al. [LYTR 13] con el fin de capturar de manera semi-automática decisiones arquitectónicas reutilizable y decisiones bajo incertidumbre usando Fuzzy Logic. Se basa en el método QOC, que, además de generar el conocimiento arquitectónico, permite generar la documentación sobre las decisiones de diseño. También mantiene trazabilidad entre decisiones arquitectónicas y diseños con el fin de detectar inconsistencias. La herramienta ADvISE (figura 2.12) utiliza un motor basado en lógica difusa (Fuzzy Inference Engine) para inferir aquellas soluciones en las que la información es difusa. También ofrece soporte de mantenimiento para la evolución de las decisiones y diseños arquitectónicos a través de la herramienta Architectural Knowledge (AK) Transformations Language, que permite interconectar las decisiones de diseño modeladas en ADvISE y los diseños arquitectónicos modelados con la herramienta VbMF (View-based Modeling Framework).

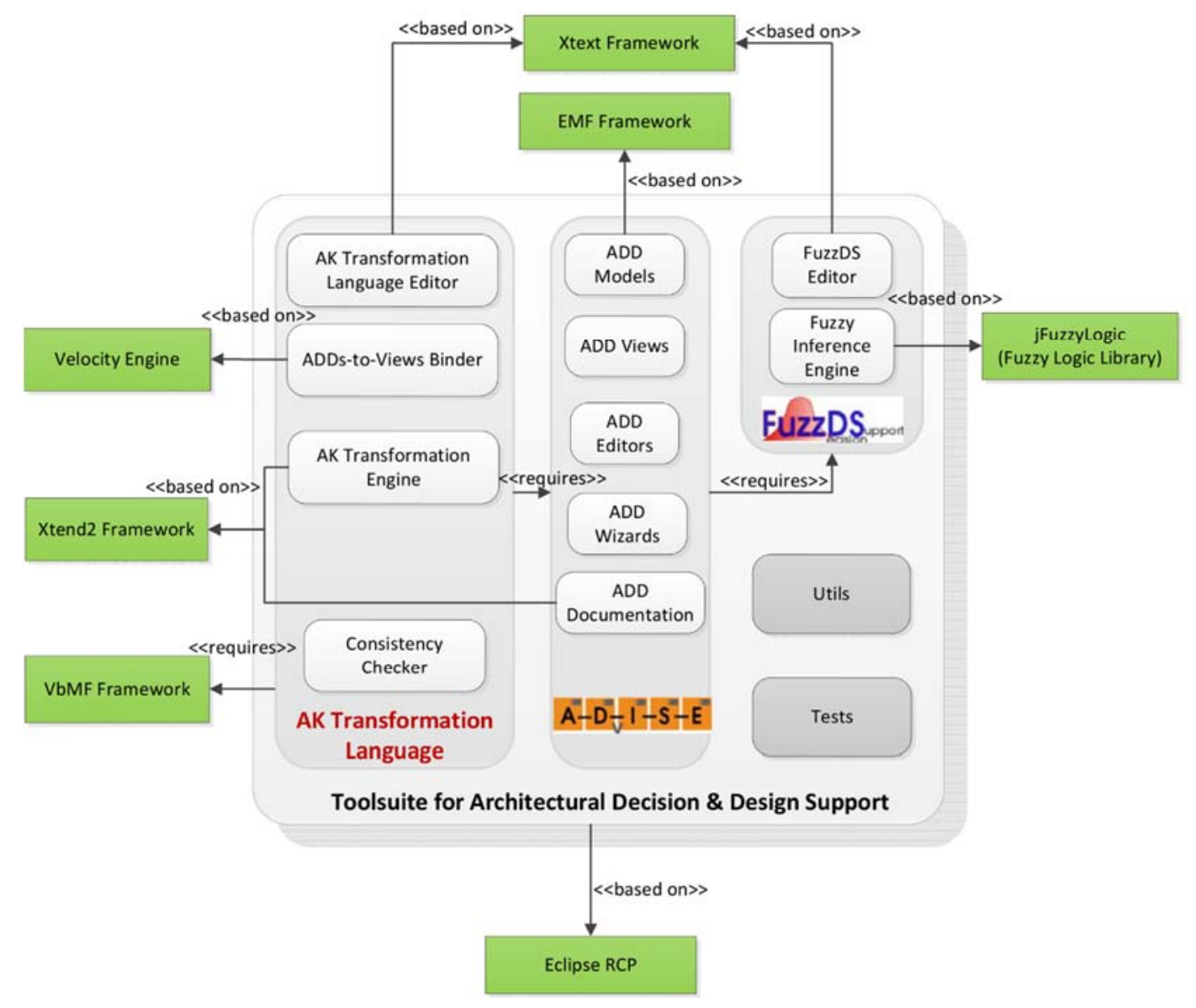

Figura 2.12: Composición de la herramienta ADvISE. 
Una mejora posterior es la herramienta CoCoADvISE (Constrainable Collaborative Architectural Design Decision Support Framework), que ofrece capacidades colaborativas en el proceso de toma de decisiones [LYTR 15A] [LYTR 15B].

Zimmermann et al. [ZIMM 15A], describe la herramienta ADMentor (desarrollado dentro de la compañía $\mathrm{ABB}$ ) que se ejecuta sobre la plataforma Sparx Enterprise Architect. ADMentor distingue entre el espacio del problema y el espacio de la solución con el fin de facilitar la toma de decisiones. La herramienta permite modelar las decisiones y modelos arquitectónicos con la misma interfaz, de manera que al igual que AREL, ambas herramientas permiten la captura de decisiones y modelado de soluciones de diseño. ADMentor soporta también el modelo QOC e incorpora edición de texto para resaltar las decisiones capturadas, como se muestra en la figura 2.13.

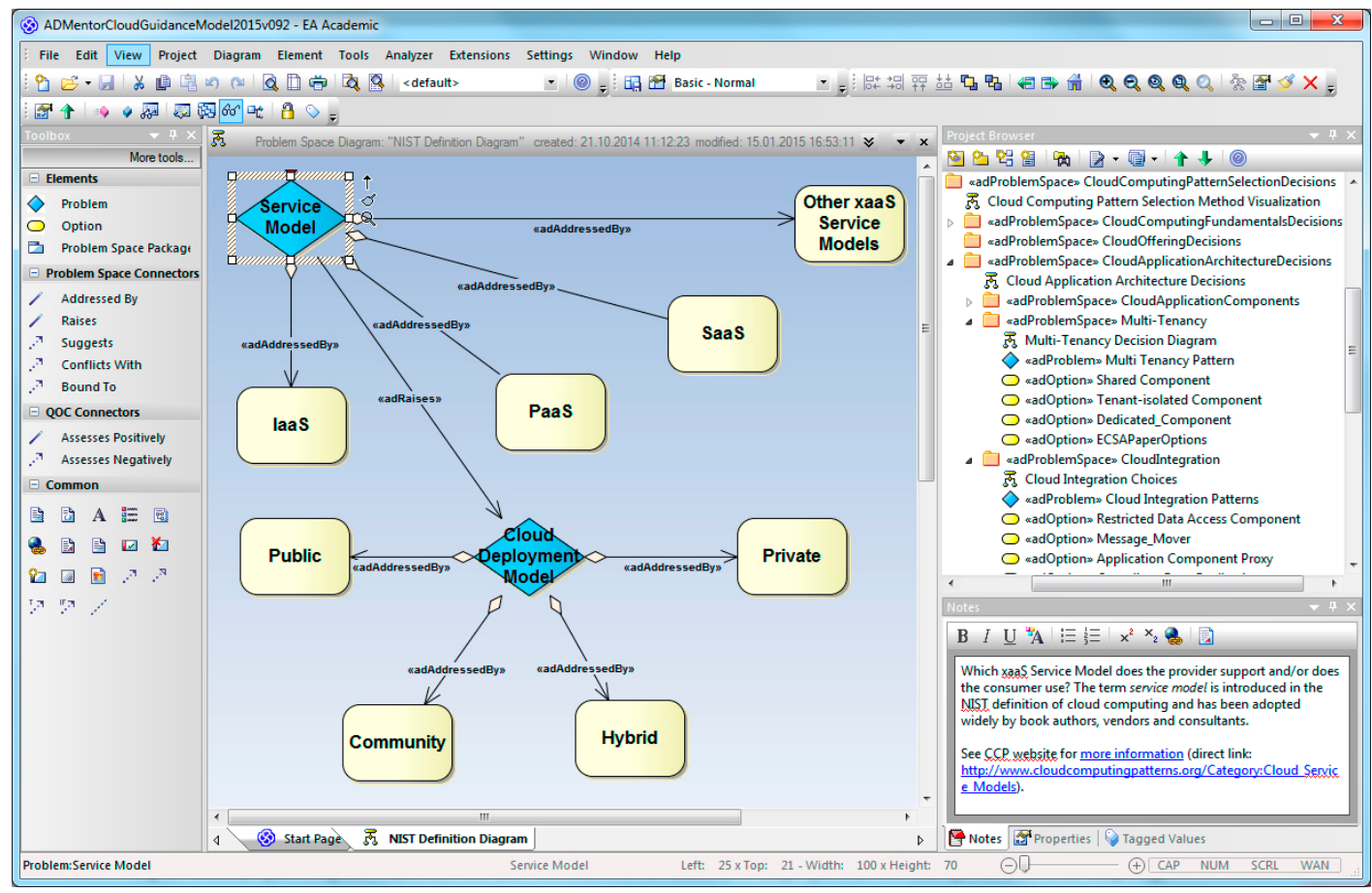

Figura 2.13: Herramienta ADMentor. Diagrama del espacio del problema, navegador del proyecto y editor de notas

Una herramienta más reciente es ArchiMedes [BOER 17], construida sobre una plataforma de gestión del conocimiento semántico WikiXL. Esta herramienta permite analizar el conocimiento arquitectónico a partir de relaciones semánticas, generadas por el usuario de la herramienta, a partir del contenido de la documentación obtenida desde repositorios. 


\subsection{Trazabilidad en Decisiones de Diseño}

Un aspecto importante en la gestión, mantenimiento y evolución de las decisiones de diseño es la trazabilidad entre los diferentes productos software y las decisiones de diseño. Por ello, la definición de enlaces de traza nos permite estimar el impacto cuando las decisiones se modifican. Uno de los problemas a la hora de establecer relaciones de traza es la carencia para representar dichas relaciones [CLEL 03] y verificar su integridad [SPAN 05], ya que muchas veces las trazas se encuentran pobremente actualizadas [LAGO 09].

La trazabilidad puede ser (i) interna entre un conjunto de decisiones de diseño y (ii) externa entre decisiones y requisitos o entre decisiones y elementos de la arquitectura software. Mediante los enlaces de traza es posible conocer tanto el origen de una mala o buena decisión como sus resultados, así como el impacto en la arquitectura debido a un cambio en los requisitos o en las propias decisiones [SPAN 05]. Además, el número de enlaces de traza entre decisiones, requisitos y arquitectura debe ser moderado y sostenible en el tiempo a medida que el sistema y su arquitectura evolucionan.

En los meta-modelos discutidos anteriormente existen propuestas que describen relaciones de trazabilidad entre requisitos, decisiones de diseño y arquitecturas. En el meta-modelo de Gilson [GILS 11], mostrado en la figura 2.14, se definen elementos de traza entre decisiones y su lógica y los requisitos significativos para la arquitectura (ASR). Los elementos de trazabilidad describen una relación entre los requisitos significativos ASR y las decisiones de diseño, referenciadas en DesignDecision y motivadas por dichos requisitos, y por otra parte la relación entre las decisiones de diseño tomadas y su resultado corresponden a la entidad Architectural ModelElement. 


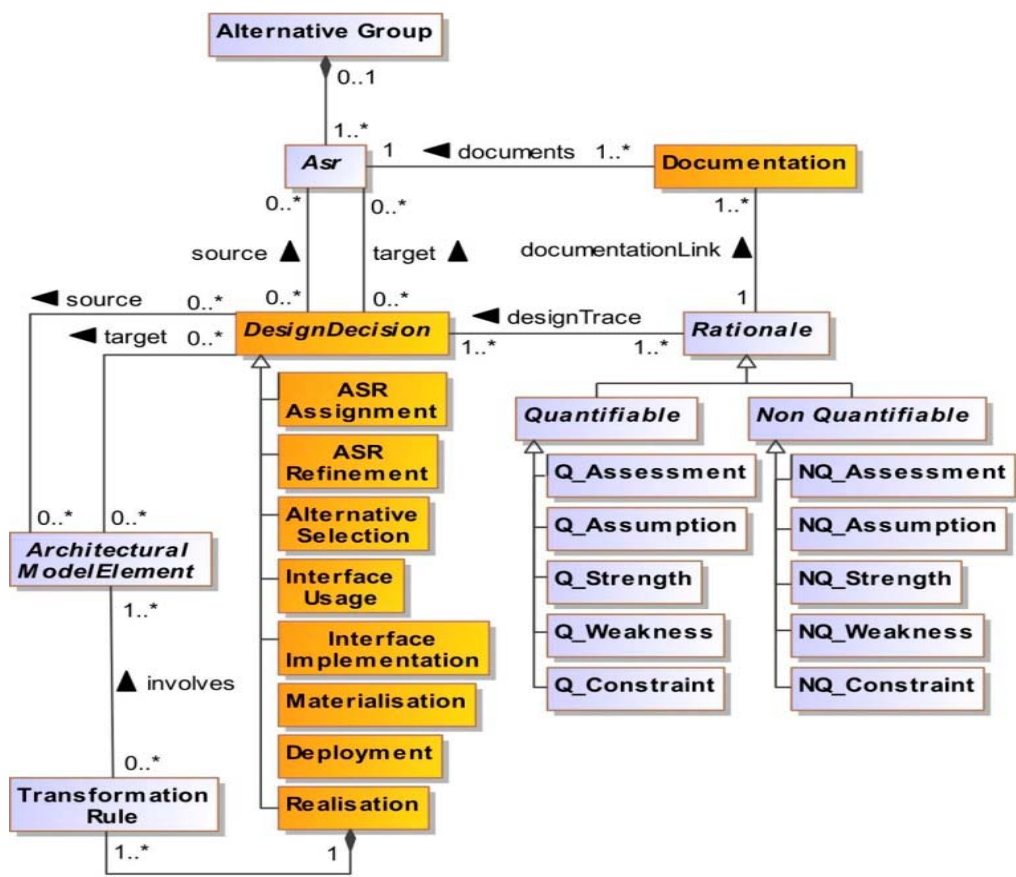

Figura 2.14. Meta-modelo de trazabilidad de la lógica de diseño.

Un modelo similar al anteriormente descrito, pero más simplificado es el propuesto en [OH 14] y que se muestra en la figura $2.15 \mathrm{y}$ en el cual la trazabilidad entre decisiones $\mathrm{y}$ requisitos se descompone en elementos de traza a requisitos funcionales, no funcionales y restricciones.

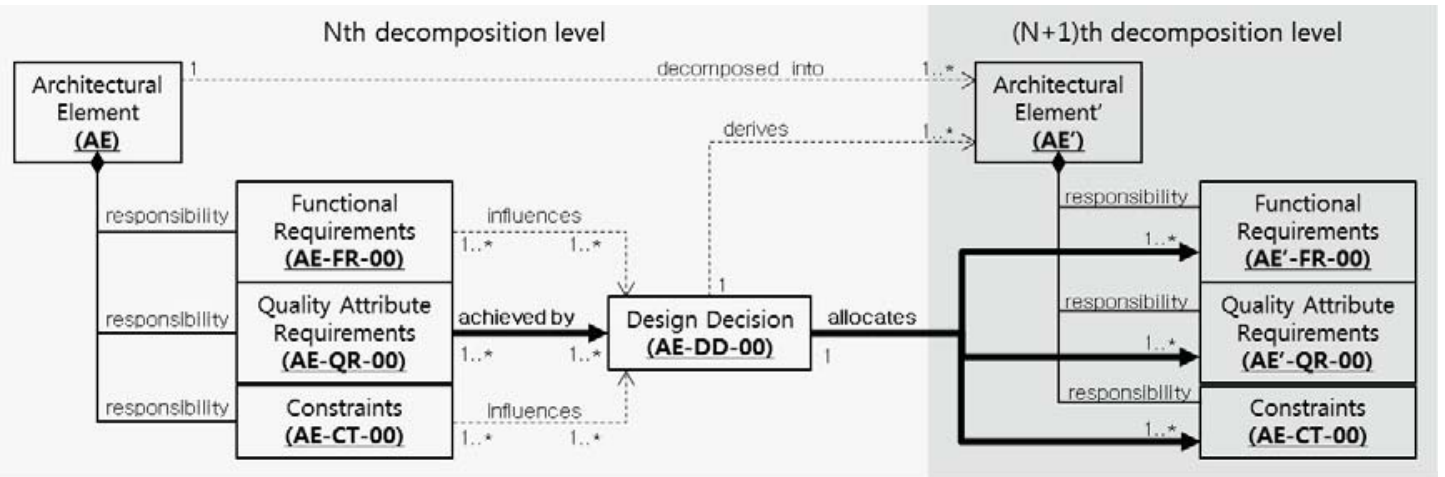

Figura 2.15. Estructura de trazabilidad en una descomposición por niveles.

Mirakhorli [MIRA 11A] propone reducir el número trazas y reutilizar el conocimiento mediante patrones de trazabilidad usando técnicas de ingeniería inversa para el establecimiento automático de las relaciones entre código y decisiones. Diversos autores resaltan la importancia de la trazabilidad para reflejar la evolución del conocimiento, [CAPI 07A]. Malavolta et al [MALA 11] se centran en los aspectos de trazabilidad bidireccional la cual permite conocer la evolución del razonamiento a lo largo del proceso de toma de decisiones y analizar como el cambio en una decisión impacta en 
las demás. Los autores presentan el meta-modelo ADDMM (figura 2.16) para describir las relaciones que guían la evolución de las decisiones, además de atributos específicos (timestamp, history y evolved) para controlar la evolución de las mismas, de manera similar a los atributos propuestos en [CAPI 07A]. Respecto a la trazabilidad bidireccional, Díaz et al. [DIAZ 11] proponen un metamodelo flexible (denominado PLAK) que soporta diferentes tipos de decisiones de diseño. Estas decisiones mantienen relaciones de trazabilidad con diferentes elementos en arquitectura de líneas de producto (PLA). Los autores proponen un método de análisis de impacto ("ripple effect") que, mediante un algoritmo de trazabilidad, devuelve un conjunto de decisiones y los elementos arquitectónicos impactados por un cambio.

Finalmente, Tang [TANG 11] señala que la gestión de la trazabilidad debe tener un carácter dinámico o adaptativo para reflejar la evolución de las especificaciones. Dado que los arquitectos software trabajan de manera simultánea, necesitan disponer de una trazabilidad documentada, flexible y actualizada. En este sentido, según Sommerville [SOMM 10], el número de elementos de traza debe ser sostenible.

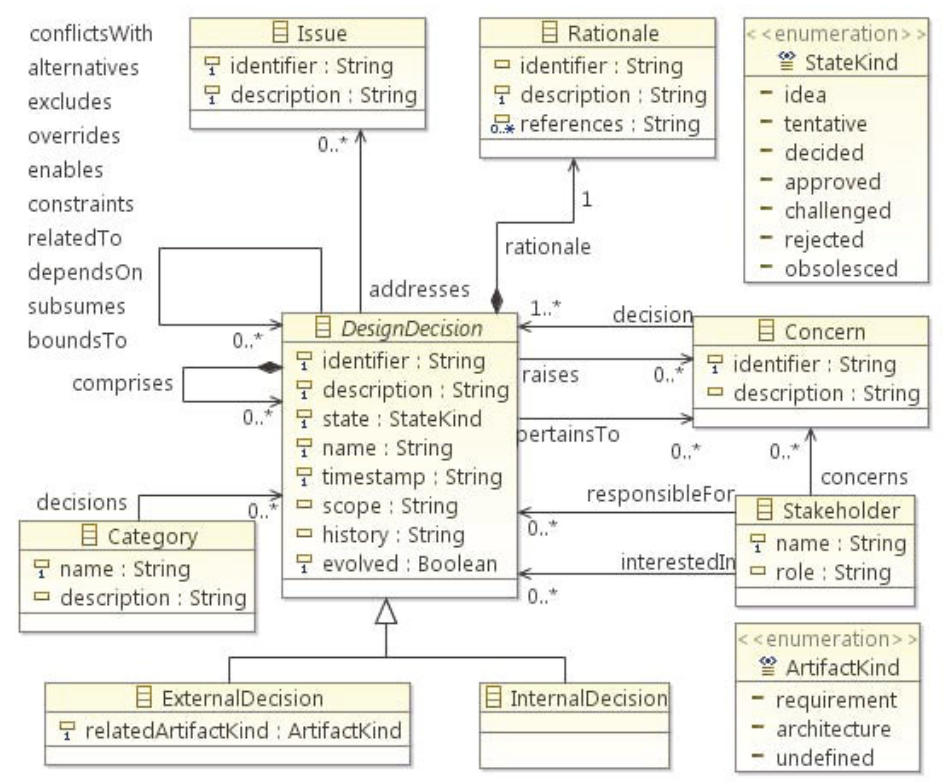

Figura 2.16 Metamodelo ADDMM.

\subsection{Evolución Sostenible en Arquitecturas Software}

A lo largo de este trabajo hemos analizado propuestas para caracterizar el conocimiento arquitectónico, donde la captura, gestión y documentación requieren un importante esfuerzo para el arquitecto software. Sin embargo, se necesita que este 
esfuerzo sea sostenible durante la evolución de la arquitectura y así limitar la cantidad de conocimiento arquitectónico a capturar y mantener. Por ello, y como eje central de esta tesis, vamos a describir en las siguientes secciones propuestas para capturar la evolución y como conseguir que esta sea sostenibilidad.

Dado que el conocimiento evoluciona a lo largo del desarrollo software, es necesario tener actualizado el conocimiento, y poder analizar y comprender los efectos de cada cambio en el conjunto de decisiones [MIRA 11B]. Con el fin de caracterizar la evolución de las decisiones de diseño, Capilla et al [CAPI 07B] definen atributos para capturar la información sobre la evolución de las decisiones (tablas II.3, II.4 y II.5 del Anexo II). Por ello se propone un meta-modelo [CAPI 11], descrito en la figura 2.17, que incorpora elementos específicos (ADOutcomeEdition, ADRuntimeElement y ADRuntimeArtifact) que permitan gestionar la evolución de las decisiones de diseño.

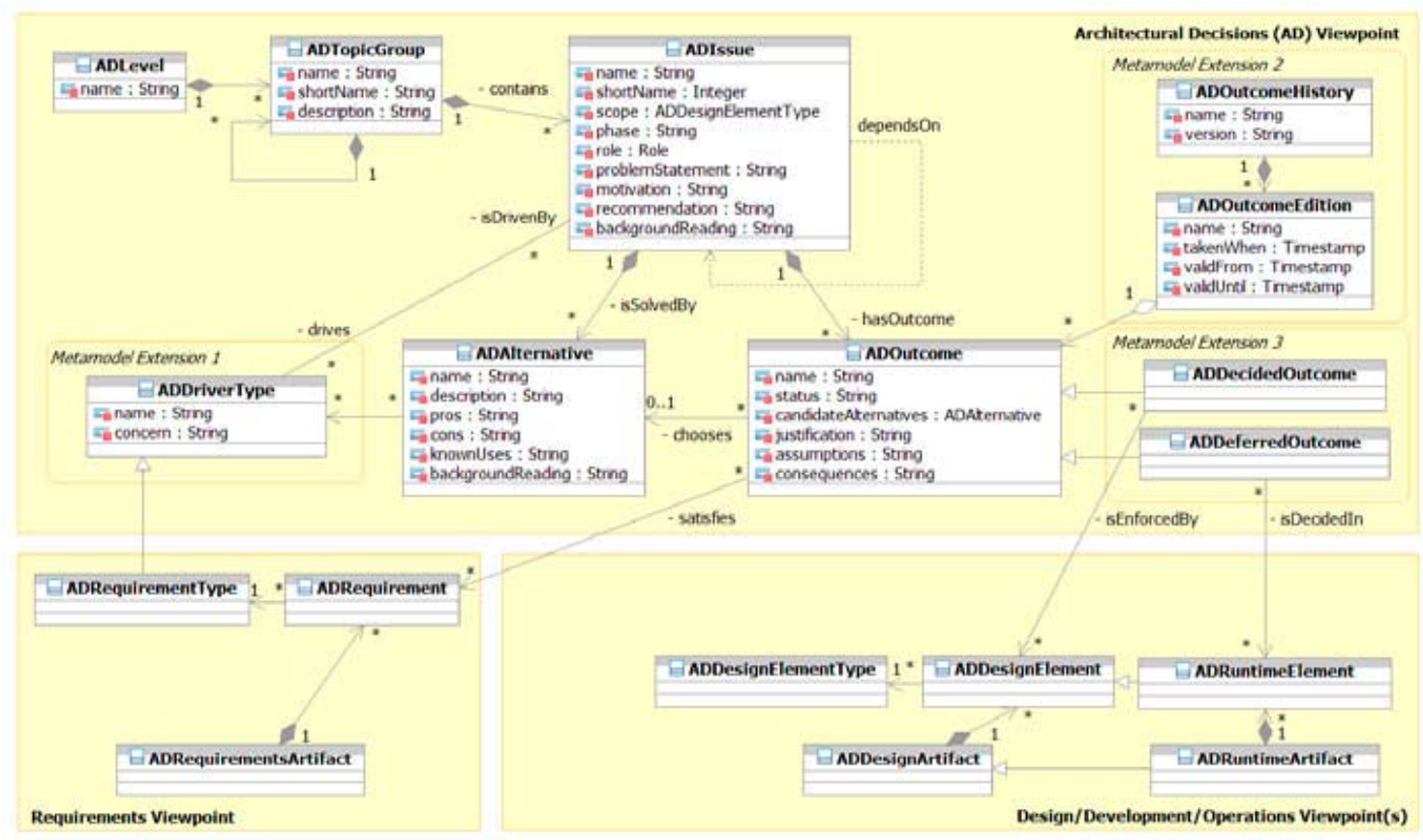

Figura 2.17 Meta-modelo para capturar decisiones de diseño arquitectónico.

Resulta importante que la evolución forme parte del conocimiento arquitectónico. Por ello, Cuesta et al. [CUES 13] proponen capturar las decisiones y los patrones que guían la evolución en la arquitectura para documentar los cambios que se producen durante su evolución. Los autores definen el concepto de "evolution style" para almacenar tanto la información de las decisiones que cambian como de los cambios producidos. Es más, también es importante que la evolución del conocimiento arquitectónico no esté en conflicto con el conocimiento ya existente. Por ello, en [GERD 15] se propone un modelo 
que analiza los posibles cambios en la arquitectura y minimice el esfuerzo en documentar los cambios [GERD 16].

Uno de los aspectos relevantes durante la evolución de un sistema es mantener actualizada la arquitectura software. Por ello, la arquitectura, sus decisiones de diseño y los cambios producidos en ellas deben perdurar en el tiempo y por ello resulta necesario medir la longevidad de los sistemas, diseños y las decisiones capturadas. En este sentido, Lago et al. [LAGO 15] plantean diferentes dimensiones para medir la sostenibilidad, entre las se encuentra la denominada sostenibilidad técnica (figura 2.18). Los autores señalan la necesidad de que el arquitecto aplique criterios de evaluación de la sostenibilidad, pero no proponen métricas para ello.

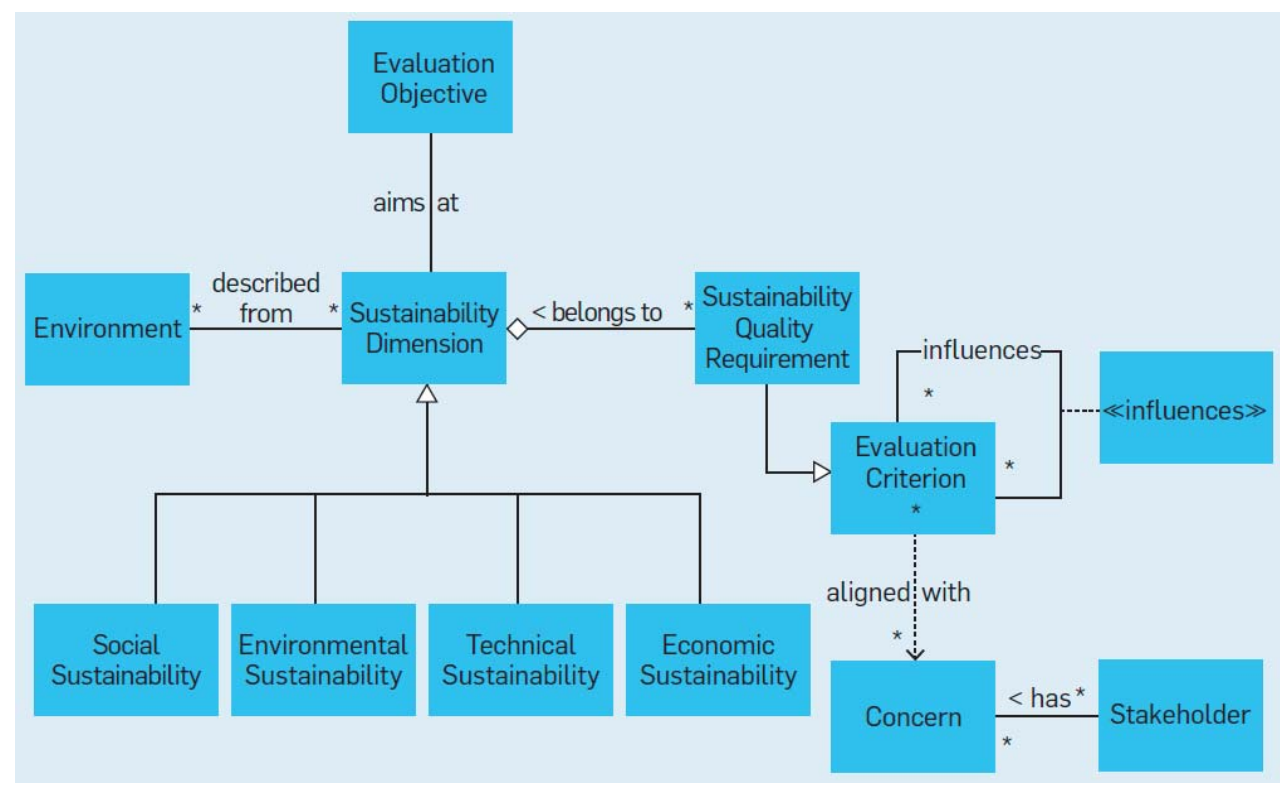

Figura 2.18 Modelo para la sostenibilidad de los requisitos de calidad software.

Con el fin de medir la sostenibilidad en los sistemas, Koziolek [KOZI 11] sugiere que una arquitectura es sostenible si el coste de su mantenimiento es rentable a lo largo de su ciclo de vida, y también que los atributos de calidad deben influir en el diseño de la arquitectura. La idea de que una arquitectura sostenible es aquella que optimiza sus recursos y es perdurable en el tiempo centra la propuesta de Calero et al. [CALE 13], para los que sostenibilidad es el grado por el que un producto software puede ser modificado, adaptado, y reutilizado con el fin de mejorarlo. Además, los autores relacionan sostenibilidad con algunos de los atributos de calidad establecidos en el estándar ISO/IEC 25010 [ISO 25010:11]. 
En esta misma línea, Venters et al. [VENT 13] señala qué atributos de calidad influyen notoriamente en la sostenibilidad de un sistema software. Así mismo, Villa et al. [VILL 16] presentan un conjunto de atributos de calidad que definen sostenibilidad en el ámbito de arquitecturas software, y que se corresponde con la Figura 2.19. Los autores vinculan la sostenibilidad del entorno definida en [BECK 14] [LAGO 15], para medir la longevidad de los sistemas mediante atributos de calidad.

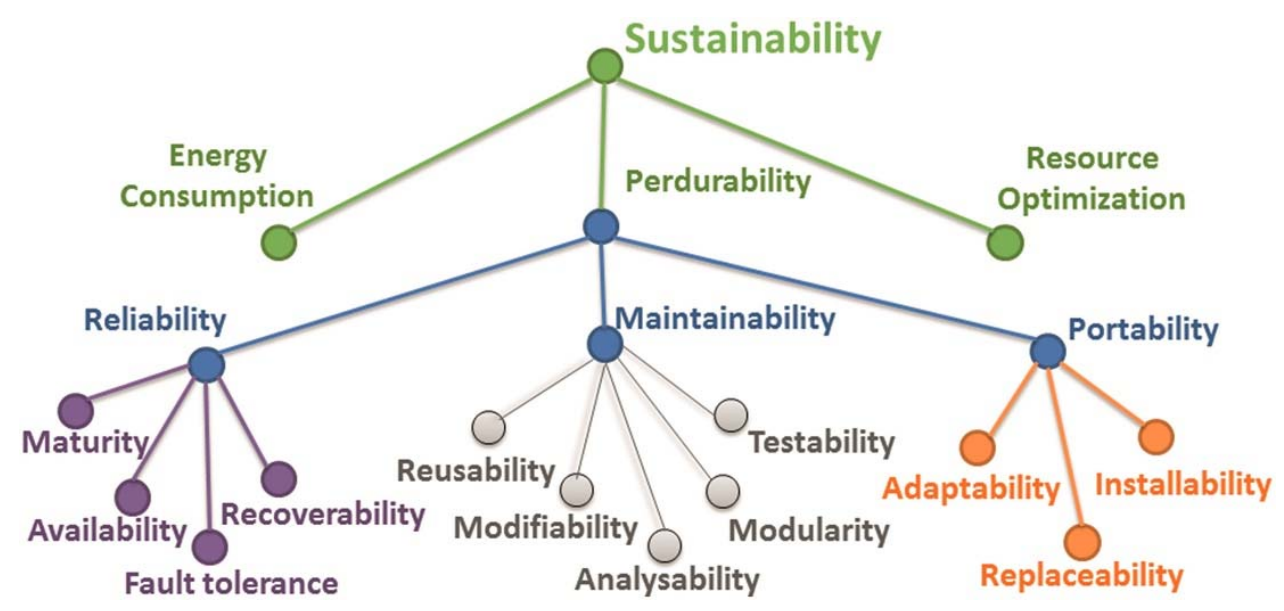

Figura 2.19. Conjunto de atributos de calidad para sostenibilidad.

A lo largo de esta sección hemos analizado algunas propuestas centradas en la importancia de la sostenibilidad en la evolución de los sistemas y la arquitectura software, pero no sugieren medidas para estimarla, tal y como discutimos en el siguiente apartado.

\subsubsection{Deuda técnica en arquitectura software}

Es de sobra conocido que la calidad de una arquitectura software se evalúa en base a atributos de calidad. En aquellos casos en los que la calidad del sistema decae, se origina una deuda técnica derivada de malas prácticas de diseño y codificación, o bien de malas decisiones de diseño incorrectamente tomadas [CUNN 92]. El uso de malas prácticas de diseño se puede detectar mediante los denominados "Architecture smells", que son el resultado de incumplir principios de diseño establecidos [FOWL 99] [LIPP 06] [GARC 09]. Según Snipes et al. [SNIP 12], esta deuda está presente en todas las fases del desarrollo software y una adecuada gestión de esta deuda ayuda a la sostenibilidad del sistema porque permite tomar adecuadamente las decisiones y minimizar costes y esfuerzos a lo largo del ciclo de vida software. 
Kruchten [KRUC 12A] [KRUC 12B] sitúa la deuda arquitectónica en el espacio de deuda técnica existente entre la solución obtenida y la solución ideal. Los autores indican que la deuda arquitectónica no es visible para los usuarios del producto software obtenido (figura 2.20) y que afecta principalmente a la evolución de la arquitectura durante su ciclo de vida.

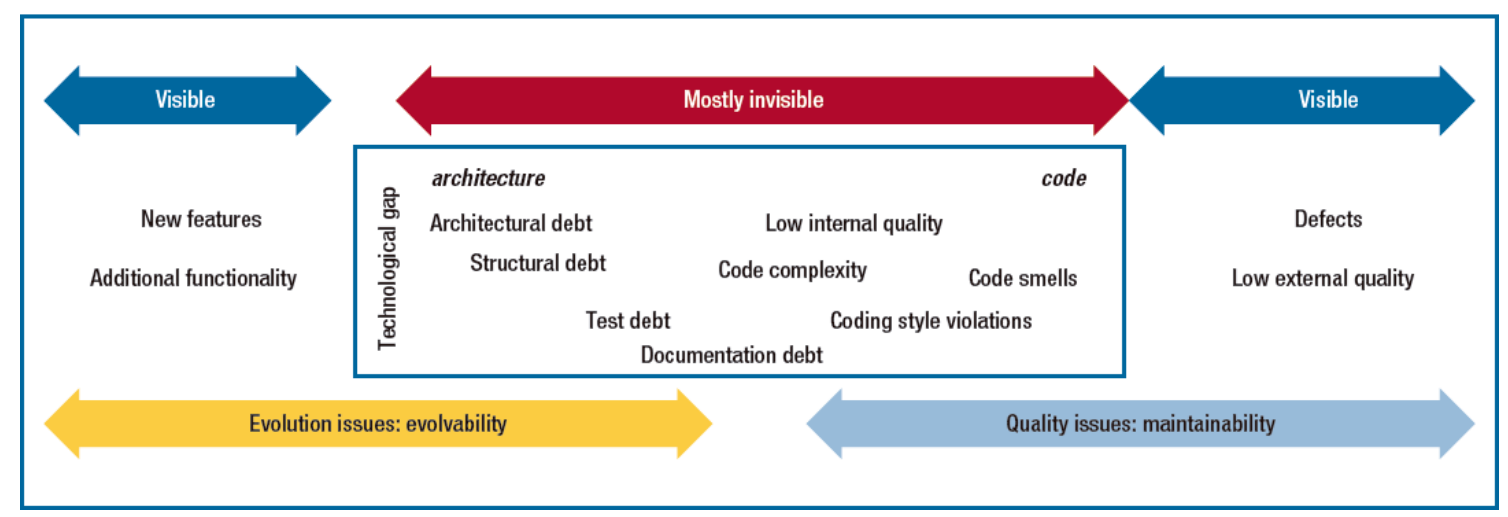

Figura 2.20: Espacio de deuda técnica.

La deuda arquitectónica puede comprometer la mantenibilidad y evolución de la arquitectura tal y como menciona Li et al. [LI 14] [LI 15]. Por ello, proponen un modelo de deuda técnica asociado a decisiones de diseño, tal y como se muestra en la figura 2.21. En dicho modelo, cada decisión de diseño incorrecta o no tomada se asocia a un elemento de deuda técnica que debe ser identificado y medido con el fin de detectar carencias en la calidad del sistema.

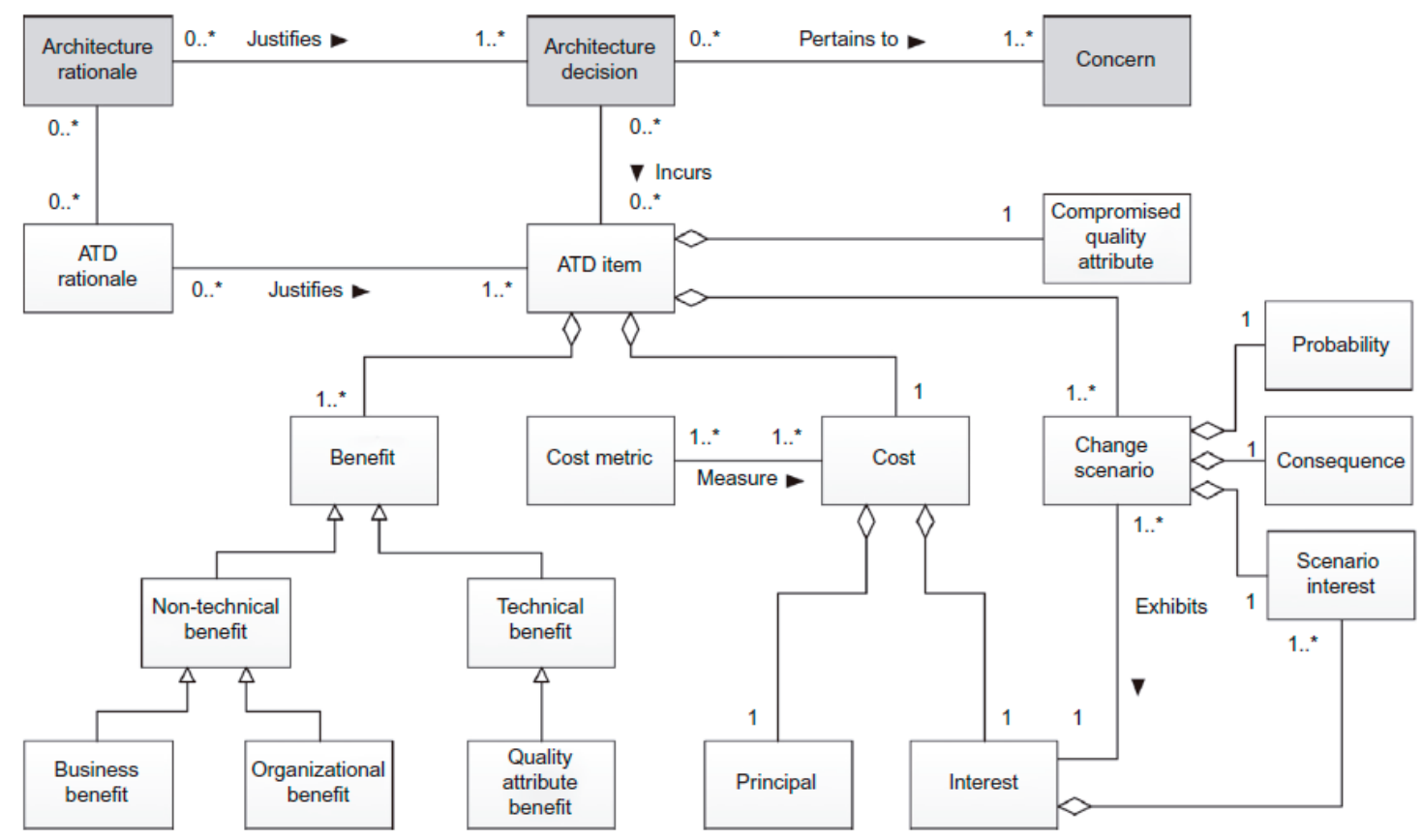

Figura 2.21 Modelo conceptual deuda arquitectónica. 
Cuando la deuda técnica es difícil de identificar o de medir, puede afectar de manera negativa a la calidad y sostenibilidad de los sistemas. Por ello Martini et al. [MART 15] proponen acotarla para limitar el impacto durante los ciclos en los que el sistema evoluciona. Otros autores [BETZ 15] sugieren que la deuda técnica afecta a la sostenibilidad de las decisiones que se toman en cada uno de los ámbitos social, económico, técnico, ambiental e individual.

\subsubsection{Estabilidad de la arquitectura y sus decisiones}

La estimación de la deuda técnica necesita de métricas adecuadas que nos permitan extender la longevidad de las decisiones de diseño y de la arquitectura. En este sentido, Riebisch et al. [RIEB 07] proponen utilizar técnicas de análisis de impacto para evaluar las consecuencias de las decisiones de diseño tomadas. Sethi et al. [SETH 09] proponen el uso de métricas para evaluar la estabilidad de los diseños, mientras que Bode et al. [BODE 10] sugieren realizar una evaluación mediante una matriz de impacto para estimar la selección de determinados principios de diseño y a qué atributos de calidad afecta. Referente a los atributos de calidad, en [SALA 16A] se propone una taxonomía para definir la estabilidad en arquitecturas software basada en cinco atributos de calidad entre los que se incluye sostenibilidad. Los autores distinguen entre la estabilidad estructural y la de comportamiento, tal y como se refleja en [SALA 16B] en el que aplican las ideas de dicha taxonomía para estimar la sostenibilidad en arquitectura cloud con capacidades de detección del contexto.

Por otra parte, Koziolek [KOZI 11] plantea el uso de métricas tanto en la fase inicial del diseño como a lo largo de su evolución. El autor realiza un estudio centrado en 40 métricas sobre su idoneidad para evaluar la sostenibilidad en la arquitectura. Sin embargo, muchas de estas métricas están enfocadas en medir código mientras que en [KOZI 13] Koziolek propone métricas específicas para medir la calidad del diseño, (Ej: "Module Interaction Stability Index" y “Module Interaction Index") y que puedan ser utilizadas para estimar cuanto de sostenible es una arquitectura.

De manera complementaria, Ampatzoglou et al. [AMPA 15] señalan que para estimar la sostenibilidad de los sistemas software es necesario predecir cómo afectará un cambio en las decisiones, y para ello proponen el uso de la métrica Instability, que mide la probabilidad a priori de la estabilidad de la arquitectura durante su evolución. Además, 
los autores señalan la necesidad de usar técnicas de evaluación del cambio a posteriori (“change proneness") para estimar qué elementos han cambiado una vez que el cambio ya se ha producido. La combinación de métricas para determinar la estabilidad y el impacto de los cambios de un sistema tanto a priori como a posteriori constituye una solución interesante que nos permite medir la estabilidad o inestabilidad de un sistema y sus arquitecturas, y así poder determinar qué cambios afectan más a la sostenibilidad.

Por último, a pesar de que las decisiones de diseño son consideradas actualmente entidades de primer nivel, existe una carencia de métricas que nos permitan medir la longevidad o estabilidad de las decisiones que conducen a una arquitectura software y estimar el impacto de la evolución en las mismas. Zdun et al. [ZDUN 13] señalan que una arquitectura software sostenible debe basarse en modelos de captura recurrentes y en reutilización del conocimiento. Además, mencionan que la sostenibilidad está relacionada con la reducción de la cantidad de información a gestionar. Entre otros criterios, los autores proponen tener en cuenta la cantidad de información a capturar por decisión, el número de dependencias entre ellas y si las decisiones están dirigidas desde los requisitos hacia una solución alcanzable y realista. En este sentido, Sehestedt et al. [SEHE 14], sugiere incluir decisiones de diseño y su lógica para medir aspectos de calidad en la arquitectura, entre ellos la sostenibilidad. Más recientemente, Zimmermann [ZIMM 15B] propone cuantificar el número de decisiones tomadas frente a las documentadas para resolver problemas de diseño, y medir el número de decisiones alternativas.

A modo de resumen, podemos señalar que la estimación de la sostenibilidad en los sistemas y sus arquitecturas como el resultado de una combinación de atributos de calidad es todavía un área de investigación emergente y que necesita de nuevas métricas específicas para estimar la sostenibilidad técnica. Además, existe una carencia visible de qué métricas son adecuadas para estimar cuanto de sostenibles son las decisiones de diseño capturadas, tal y como resumimos en las conclusiones al estado del arte.

\subsection{Conclusiones}

El estado del arte descrito refleja la importancia de las decisiones de diseño en arquitecturas software, así como los esfuerzos de diversos autores para construir herramientas que permitan capturar, documentar, gestionar y utilizar este conocimiento 
arquitectónico, como un valor añadido a la documentación tradicional basada en vistas arquitectónicas.

La captura de decisiones de diseño y su lógica resulta de suma importancia para reducir los esfuerzos del mantenimiento de los sistemas y permitir comprender y recrear las decisiones tomadas en aquellos en los que la documentación es inexistente u obsoleta, o bien cuando los creadores del sistema no se encuentren disponibles.

Los meta-modelos propuestos para representar las decisiones de diseño carecen de una estructura suficiente flexible para facilitar la evolución del conocimiento arquitectónico. Además, no sugieren formas de medir la calidad de las decisiones de diseño y su impacto en la arquitectura, ni cómo se debe cuantificar la cantidad ideal de decisiones a capturar.

En este sentido, no hay un consenso en cómo caracterizar una arquitectura software sostenible y solamente algunas propuestas limitan el conjunto de decisiones a capturar. Por ello, con el fin de garantizar la calidad de los sistemas y medir como de sostenible son, son necesarias soluciones que nos permitan medir como de sostenible es una arquitectura y las decisiones que motivan la selección de un determinado diseño. Dado que los aspectos de calidad son múltiples, es importante centrarse en aquellos que afecten de manera más directa a la calidad del conocimiento arquitectónico capturado. Finalmente, la estimación del impacto cuando las decisiones cambian resulta clave para medir la estabilidad del sistema y como perduran las mejores decisiones en el tiempo.

Para concluir, podemos afirmar que existe una carencia de métricas que nos permitan medir de manera efectiva la sostenibilidad, longevidad y estabilidad de las decisiones de diseño y su arquitectura. 


\section{Capítulo 3. Planteamiento del Problema}

Tal y como se ha comentado en las conclusiones del capítulo anterior (sección 2.6), no existe actualmente un consenso ni métodos adecuados para la gestión sostenible de decisiones de diseño en arquitecturas software ya que la estructura rígida de los metamodelos propuestos no facilitan esta tarea. Además, tampoco existen un consenso para definir cuanta cantidad de conocimiento arquitectónico es necesario capturar ni cuantos atributos deben caracterizar a las decisiones de diseño para que su gestión sea sostenible. Por ello, la necesidad de definir cuanto conocimiento arquitectónico es necesario capturar, influye de manera especial en el coste que es necesario emplear para documentar las decisiones de diseño.

De manera complementaria es importante conoce cuantas relaciones de trazabilidad deben capturarse entre decisiones, requisitos y arquitecturas, así como las propias decisiones. Por ello, el número de elementos de traza en una red de decisiones influye en la cantidad de enlaces a mantener y en la complejidad de la propia red, ya que a mayor complejidad más difícil puede resultar su evolución en el tiempo.

Tampoco existe un consenso en cómo deben evolucionar las decisiones de diseño durante el ciclo de vida de un sistema, ni en cómo se debe evaluar el impacto cuando las decisiones se modifican. A pesar de que los cambios afectan a la estabilidad de la red de decisiones de diseño, tampoco hay un acuerdo de cómo evaluar el impacto de aquellas decisiones que cambian más frecuentemente, ni de cómo estimar el impacto de estos cambios en la estabilidad del conocimiento arquitectónico.

Todos estos desafíos necesitan de estimadores y métricas adecuadas que nos permitan evaluar como de sostenible resulta mantener el conocimiento asociado a las decisiones de diseño en Arquitectura Software. Estas métricas son necesarias para medir los aspectos de calidad tanto en la arquitectura como en el propio conjunto de decisiones. De manera complementaria, las métricas estimadas de manera individual no proporcionan en muchos casos indicadores precisos de los diferentes aspectos de calidad y entre ellos de la sostenibilidad de las arquitecturas y de las decisiones. 
Por los motivos expuestos anteriormente, el problema que vamos a tratar en este trabajo de investigación es:

\section{"Diseñar un modelo y proponer métricas que nos permitan evaluar \\ la sostenibilidad de un conjunto de decisiones de diseño en arquitecturas software"}

Visto este planteamiento general, los subproblemas que vamos a abordar en este trabajo son:

1. Identificar qué aspectos de las decisiones de diseño son evaluables desde el punto de vista de la sostenibilidad en relación con su arquitectura software.

2. Definir criterios para medir dicha sostenibilidad en base a atributos de calidad.

3. Cuantificar el tamaño adecuado de un conjunto de decisiones de diseño para que la arquitectura software sea más sostenible.

4. Establecer mecanismos para evaluar el impacto producido por los cambios en las decisiones.

Los subproblemas anteriormente descritos cubren de manera precisa la problemática sobre la necesidad de contar con un modelo y estimadores adecuados para proporcionar una solución sostenible al conocimiento arquitectónico. En el siguiente capítulo de este trabajo propondremos una solución a cada uno de estos subproblemas. 


\section{Capítulo 4. Solución Propuesta}

Una vez descrito el planteamiento del problema y subproblemas concretos, pasamos a exponer la solución al problema planteado. Las etapas principales que vamos a cubrir con nuestra solución son las siguientes:

1. Diseñar un modelo que nos permita caracterizar las decisiones de diseño en arquitecturas software de manera sostenible.

2. Definir unos criterios que relacionen los aspectos de calidad y elementos sostenibles del modelo con aquellas métricas que puedan ser útiles para medir diferentes aspectos de sostenibilidad en decisiones de diseño.

3. Proponer e implementar métricas que permitan estimar la sostenibilidad de las decisiones de diseño en arquitectura software.

Además, para el desarrollo de nuestra solución, contaremos con las limitaciones que a continuación detallamos

\subsection{Hipótesis de trabajo.}

Como paso previo a la solución, vamos a poner unas limitaciones a este trabajo que se describen en forma de hipótesis de trabajo, tal y como describimos a continuación:

1. Representación del conocimiento arquitectónico El conocimiento arquitectónico, correspondiente al diseño de un sistema, lo representaremos mediante un grafo en el que cada nodo representa una decisión de diseño y los enlaces representan relaciones con otras decisiones.

2. Granularidad de decisiones de diseño.: En este trabajo limitamos el nivel más fino de granularidad de las decisiones al nivel de clase.

3. Tipo de grafo. Los modelos de grafos que utilizaremos en este trabajo será dirigido y estará representado por la direccionalidad de los enlaces de decisiones. Consideraremos grafos tantos cíclicos como acíclicos.

Para analizar los impactos que se produce por cambios en las decisiones existentes, definimos las siguientes restricciones: 
4. Tipo de impacto a analizar. En este trabajo solo consideramos el cambio producido en una decisión, y excluimos la inclusión y/o eliminación de decisiones.

5. Evaluación del impacto en el conocimiento. Nuestra propuesta considera el impacto de cambios solo en el conjunto de decisiones de diseño, y excluimos los impactos en requisitos y en la solución arquitectónica.

6. Peso de las relaciones entre decisiones. No consideraremos pesos en las relaciones entre decisiones a efecto de cálculo de distancia.

\subsection{Modelo Sostenible para la Gestión de Decisiones de Diseño}

En la actualidad, existe una carencia de procesos relevantes asociados a la captación y gestión de las decisiones de diseño, y sus relaciones de trazabilidad, orientados a garantizar la sostenibilidad de dichas decisiones y su trazabilidad. Además, existe una falta de acuerdo para producir un modelo o meta-modelo común, tanto para la adquisición de las decisiones de diseño como de las relaciones de trazabilidad entre los diferentes elementos arquitectónicos. Por ello, vamos a proponer un modelo de gestión sostenible del conocimiento arquitectónico que tenga en cuenta la evolución dinámica de dicho conocimiento.

Con el objetivo de proponer nuestro modelo, vamos a definir el concepto de arquitectura software sostenible, aplicada a la gestión de decisiones de diseño arquitectónico.

Definición: Arquitectura software sostenible es "aquella que incorpora su evolución, con el menor impacto posible en el conocimiento ya adquirido y sin aumentar el esfuerzo necesario para gestionar dicho conocimiento".

De la definición anterior se deduce que una arquitectura software es sostenible si el mantenimiento del conocimiento ya adquirido y su evolución son sostenibles. El esfuerzo necesario para capturarlo debe ser proporcional al número de elementos que forman dicho conocimiento y la relevancia de las decisiones tomadas. Cuando el esfuerzo es elevado, el arquitecto puede no incluir la totalidad del conocimiento arquitectónico produciéndose perdida del mismo. Además, la arquitectura debe estar dimensionada para incluir su propia evolución, y esta inclusión no deberá afectar negativamente al resto de decisiones 
de diseño. En nuestra propuesta de arquitectura software sostenible formalizaremos un meta-modelo, basado en el modelo de arquitectura software definido en [ISO 42010:11]. En dicho meta-modelo detallaremos los elementos que caracterizan nuestro modelo de sostenibilidad. También propondremos métricas para estimar la complejidad del conocimiento arquitectónico adquirido, y el esfuerzo necesario para capturar las decisiones de diseño por parte de los arquitectos. Además, estableceremos mecanismos para estimar el impacto de un cambio en el conocimiento arquitectónico. A continuación, presentaremos nuestra propuesta de meta-modelo para la gestión sostenible del conocimiento.

\subsubsection{Meta-modelo para la gestión sostenible de decisiones de diseño}

Para dar solución a los problemas planteados en el capítulo 3, y teniendo en cuenta los meta-modelos analizados en el capítulo 2 de este trabajo, proponemos un metamodelo ${ }^{1}$ configurable y adaptable a las necesidades de cada Stakeholder para (i) capturar el conocimiento arquitectónico adecuado a las necesidades de cada usuario mediante plantillas configurables, (ii) facilitar el mantenimiento de las redes de diseño y (iii) estimar de manera más precisa la sostenibilidad de las decisiones de diseño cuando estas cambian. Con este meta-modelo controlamos mejor la longevidad y estabilidad de las decisiones de diseño y proporcionamos mecanismos para definir qué cantidad de conocimiento resulta más adecuada capturar

El meta-modelo que proponemos (figura 4.1) ha sido introducido por el autor de este trabajo en [CARR 15]. Nuestra propuesta está formada por los paquetes (i) Architecture Model, que representa el modelo de arquitectura software definido en [ISO 42010:11], (ii) $\mathrm{DD}$ Core $^{2}$, que define el modelo de decisiones de diseño, (iii) $\mathrm{DD}$ Extensions, que complementa de manera sostenible el conocimiento adquirido por DD Core, y (iv) Sustainability of the Design Decision Model, que define y calcula la sostenibilidad del conocimiento arquitectónico gestionado.

\footnotetext{
${ }^{1}$ La existencia de clases no conectadas en el meta-modelo permite su expansión de manera flexible y configurable, al no ser estrictamente obligatorio su uso.

${ }^{2}$ Se declaran listas de valores asociados a atributos definidos en entidades de este paquete.
} 


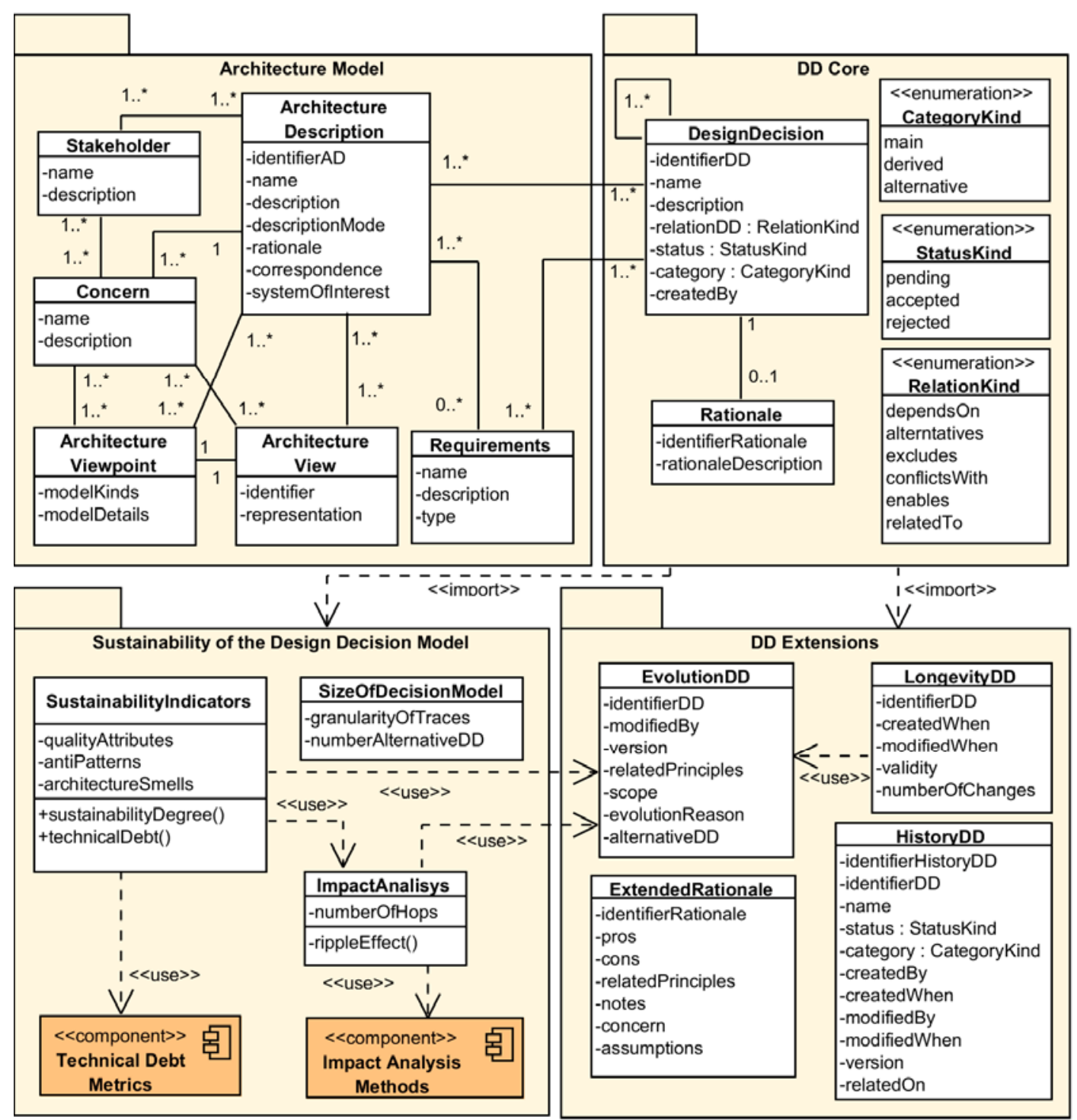

Figura 4.1: Meta-modelo propuesto para la gestión sostenible de decisiones de diseño.

A continuación, describimos cada uno de los paquetes que forman este metamodelo. Con el fin de centrar al lector en la descripción de cada paquete, minimizaremos gráficamente el resto de paquetes para destacar el paquete objeto de descripción.

El paquete Architecture Model tiene como referencia el modelo conceptual para la descripción arquitectónica definido en el estándar [ISO 42010:11]. Proponemos una simplificación de dicho modelo, porque consideramos que algunos elementos definidos en el estándar se pueden incluir como atributos de otros elementos. En la figura 4.2 mostramos las clases (i) Architecture Description, para capturar las descripción arquitectónica que forman la arquitectura, (ii) Stakeholder para identificar a los responsables del desarrollo descriptivo de la arquitectura, (iii) Concern para resaltar los aspectos de interés en un sistema y que son relevantes para cada Stakeholder, (iv) 
Architecture View para reflejar las vistas que un Stakeholder puede utilizar para organizar las descripciones, (v) Architecture Viewpoint para registrar el punto de vista que cada Stakeholder tiene de la arquitectura, y (vi) Requirements para detallar los requisitos funcionales y no funcionales de la arquitectura.

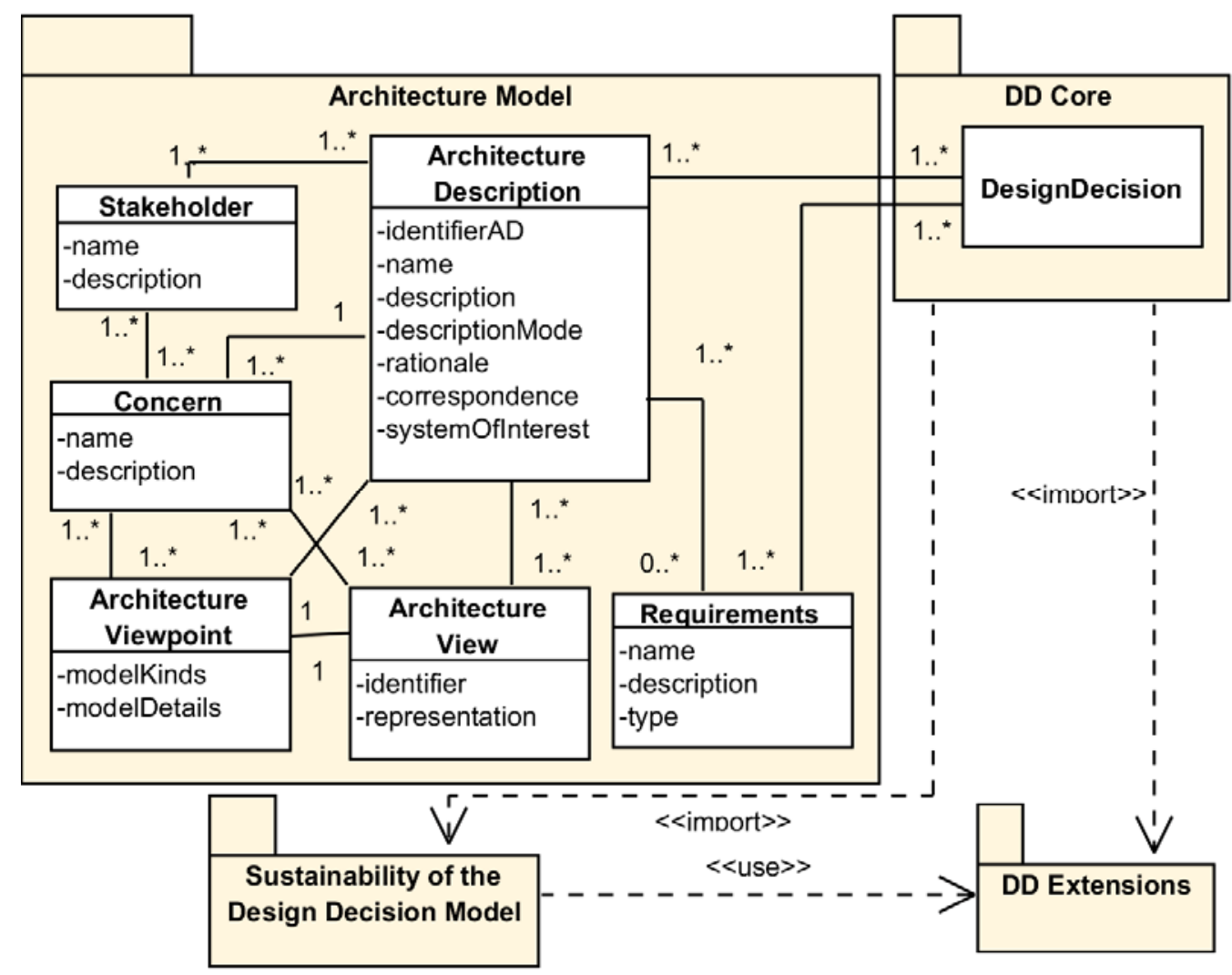

Figura 4.2: Paquete "Architecture Model".

La clase Architecture Description ${ }^{3}$ identifica cada descripción arquitectónica por el atributo identifier $A D$, junto con el atributo name y una explicación de la misma en description. En nuestro objetivo de simplificar el modelo descrito en [ISO 42010:11], incorporamos (i) la clase System del estándar en el atributo systemOfInterest para identificar al sistema cuya arquitectura estamos describiendo, (ii) la clase Architecture Rationale del estándar en el atributo rationale para definir la lógica que justifica la decisiones de diseño y (iii) la clase Correspondence del estándar en el atributo correspondence para reflejar las relaciones de interés dentro de (o entre) descripciones arquitectónicas. Las vistas (Architecture View) y los puntos de vista arquitectónicos (Architecture Viewpoint) no se modifican respecto al estándar y al concepto tradicional

\footnotetext{
${ }^{3}$ La clase Architecture Description representa cualquier elemento arquitectónico.
} 
de arquitectura software. La clase Requirements identifica los requisitos funcionales y no funcionales de la arquitectura, y que motivan las decisiones de diseño.

El paquete DD Core ${ }^{4}$ (figura $4.3^{5}$ ) permite capturar las decisiones de diseño. Teniendo en cuenta las conclusiones expresadas en el capítulo 2, y con el objetivo de alcanzar una gestión sostenible de las decisiones de diseño, proponemos (i) reducir el número de clases para capturar las decisiones de diseño, (ii) incorporar las clases afectadas como atributos de otras clases existentes, y (iii) clasificar y separar los atributos de decisiones de diseño aplicando criterios de sostenibilidad. Para ello, creamos un subconjunto de atributos básicos ubicados en las clases de este modelo, y el resto de atributos estarán en las clases del paquete DD Extensions, y la dependencia entre estos paquetes está representada por una relación de importación reflejada en la figura 4.3. De esta manera, cada Stakeholder puede considerar a cada clase ubicada en el paquete $D D$ Extensions, y a sus atributos, como un elemento opcional cuyo uso influye en la gestión sostenible del conocimiento arquitectónico.

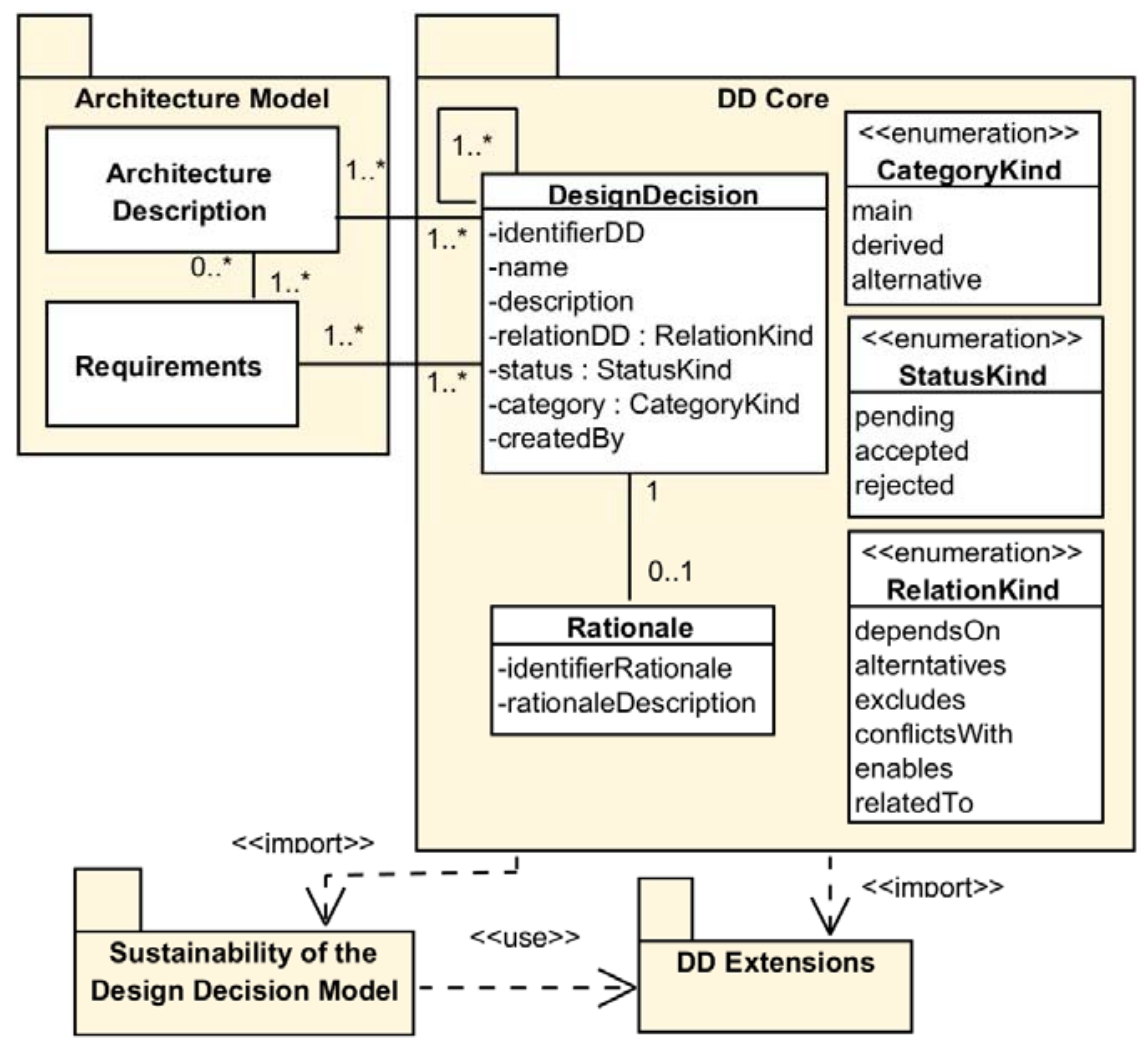

Figura 4.3: Paquete "DD Core".

\footnotetext{
${ }^{4}$ Se declaran listas de valores asociados a atributos definidos en entidades de este paquete.

${ }^{5}$ La clase Architecture Description representa cualquier elemento arquitectónico
} 
Además, para gestionar de manera sostenible el conocimiento arquitectónico, el paquete $D D$ Core se apoya en los cálculos de sostenibilidad ofrecidos por el paquete Sustainability of the Design Decision Model, y que en la figura 4.3 está representado mediante una relación de importación.

En la clase DesignDecision definimos el subconjunto de atributos (ver tabla 4.1) que definen básicamente una decisión de diseño. Con identifierDD y name identificamos a una decisión de diseño y con description aportamos su descripción. El atributo status representa el estado actual de la decisión de diseño. Aquellas decisiones obsoletas pasarán a formar parte de la clase HistoryDD ubicado en el paquete DD Extensions. Los atributos relationDD y category definen la relación existente entre decisiones y categoría que una decisión tiene frente al resto de decisiones, respectivamente. Incorporamos el atributo createdBy para identificar el Stakeholder que creo esta decisión. Las relaciones de trazabilidad existentes entre decisiones de diseño nos permitirán analizar la evolución del conocimiento, reconsiderando parte del mismo como rechazado u obsoleto. Cada decisión de diseño está relacionada con una descripción arquitectónica y al menos con un requisito, representados por las clases Architecture Description y Requirements respectivamente, y que en la figura 4.3 se indica mediante las relaciones existentes con el paquete Architecture Model.

Tabla 4.1. Atributos de la clase DesignDecision.

\begin{tabular}{|c|c|}
\hline Atributo & Descripción \\
\hline identifierDD & Identifica unívocamente a la decisión. \\
\hline name & Contiene el nombre de la decisión. \\
\hline description & Contiene la descripción de la decisión. \\
\hline relationDD & $\begin{array}{l}\text { Clasifica la dependencia entre decisiones. Pueden ser depende de (dependsOn), } \\
\text { ser alternativa de (alternatives), excluir a (excluds), estar en conflicto con } \\
\text { (conflictsWith), validar otra (enables), o estar relacionada con otra DD } \\
\text { (relatedTo). }\end{array}$ \\
\hline status & $\begin{array}{l}\text { Determina el estado actual de cada decisión. Se corresponde con aceptada } \\
\text { (pending), aprobada (accept), rechazada (rejected) }\end{array}$ \\
\hline category & $\begin{array}{l}\text { Se corresponde a la clasificación otorgada a cada decisión relacionada a si es la } \\
\text { decisión principal (main), derivada de una principal (derived) o alternativa a } \\
\text { una principal (alternative). }\end{array}$ \\
\hline createdBy & Identificador del arquitecto que toma la decisión. \\
\hline
\end{tabular}


Incluimos la clase Rationale para reflejar la motivación del Stakeholder en la elección de una decisión de diseño frente a otras, y que está intrínsecamente asociada con cada decisión de diseño. Cada Stakeholder podrá complementar esta motivación utilizando la clase extendedRationale ubicada en el paquete DD Extensions. La descripción detallada de los atributos de la clase Rationale se indican en la tabla 4.2.

Tabla 4.2. Atributos de la clase Rationale.

\begin{tabular}{l|l}
\hline Atributo & Descripción \\
\hline identifierRationale & Identifica unívocamente el motivo o razón. \\
\hline rationaleDescription & Describe los motivos o razones que justifican una decisión.
\end{tabular}

El tercer paquete de nuestra propuesta es DD Extensions (figura 4.4), y complementa el conocimiento arquitectónico gestionado por el paquete DD Core de manera sostenible. Incorpora las clases LongevityDD, ExtendedRationale, EvolutionDD, todas ellas para extender el conocimiento arquitectónico capturado, y HistoryDD para gestionar su evolución histórica. Como ya hemos indicado anteriormente, proponemos el uso de estas clases y sus atributos, como elementos opcionales que complementan de manera sostenible el conocimiento arquitectónico capturado por DD Core.

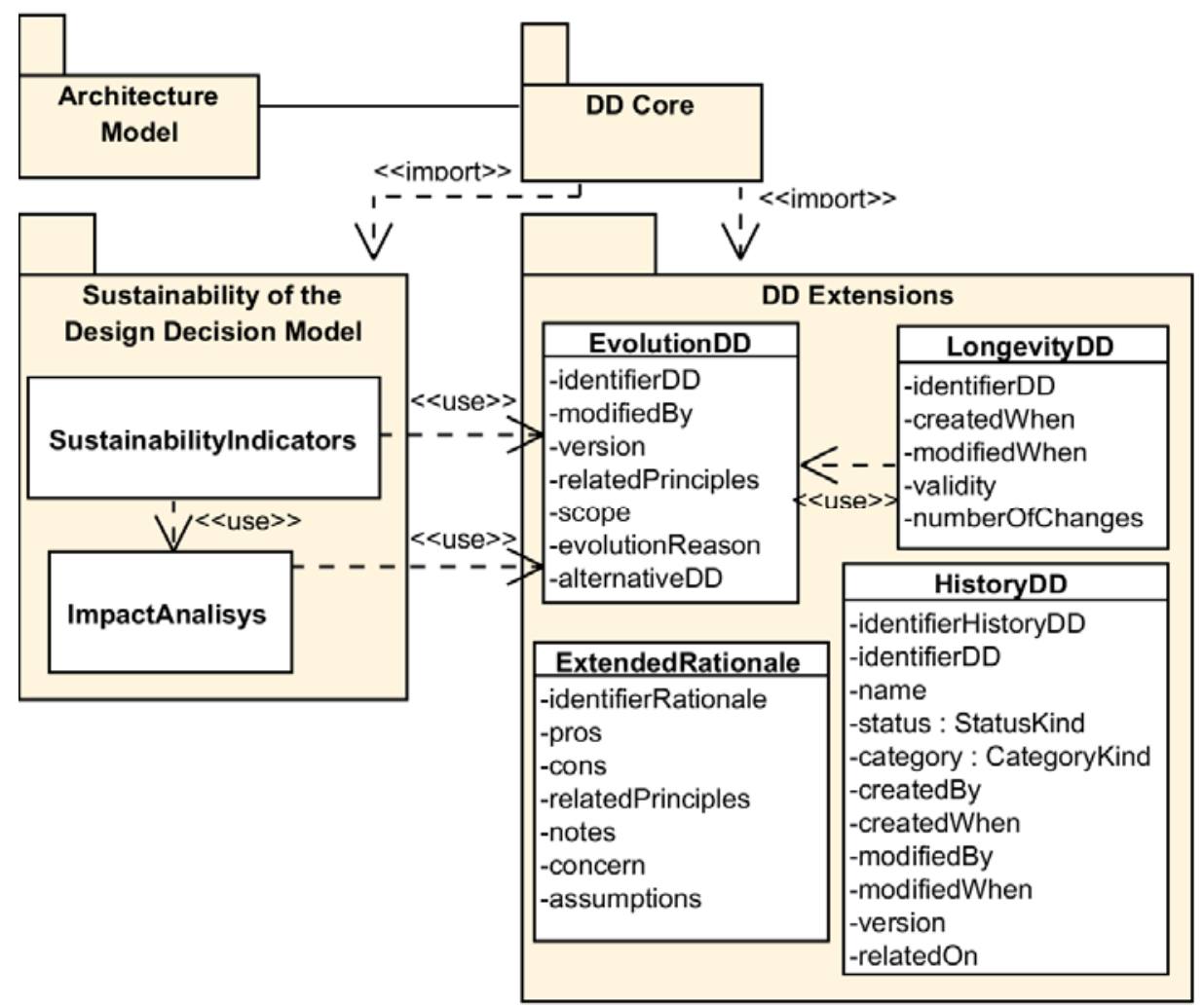

Figura 4.4: Paquete "DD Extensions". 
La clase LongevityDD contiene los atributos de las decisiones de diseño relacionados con el tiempo. Cuándo se creó (createdWhen), cuándo se modificó (modifiedWhen) una decisión. Además, permite saber cuándo una decisión puede ser considerada no valida (validity) y el número de cambios de una decisión de diseño. La descripción completa de estos atributos se indica en la tabla 4.4 .

Tabla 4.3. Atributos de la clase LongevityDD.

\begin{tabular}{l|l|}
\hline Atributo & Descripción \\
\hline identifierDD & Identificador de la decisión de diseño a la que complementa esta entidad \\
\hline createdWhen & Fecha en la que se crea la decisión. \\
\hline modifiedWhen & Fechas en las que se han modificado la decisión. \\
\hline validity & Fecha cuando la decisión podrá ser revisada \\
\hline number_ofChanges & Número de veces que una DD ha cambiado
\end{tabular}

La motivación de una decisión está reflejada por la clase ExtendedRationale. Complementa a la clase Rationale, ubicada en DD Core, con los pros (pros) y contras (cons) de esta motivación. Posee otros atributos para complementar esta motivación. En la tabla 4.3 realizamos una descripción más detallada.

Tabla 4.4. Atributos de la clase ExtendedRationale.

\begin{tabular}{|c|c|}
\hline Atributo & Descripción \\
\hline identifierRationale & $\begin{array}{l}\text { Identificador de la lógica empleada a la que complementa en la clase } \\
\text { Rationale }\end{array}$ \\
\hline pros & $\begin{array}{l}\text { Se corresponde con la evaluación favorable frente a las decisiones } \\
\text { alternativas. }\end{array}$ \\
\hline cons & $\begin{array}{l}\text { Se corresponde con la evaluación negativa frente a las decisiones } \\
\text { alternativas. }\end{array}$ \\
\hline relatedPrinciples & $\begin{array}{l}\text { Es la relación de principios de diseño que pueden ser interesantes en un } \\
\text { momento dado. }\end{array}$ \\
\hline notes & Información complementaria. \\
\hline concern & Especifica las restricciones impuestas por los requisitos sobre la decisión. \\
\hline assumptions & $\begin{array}{l}\text { Permiten conocer los fundamentos en los que se basa la toma de } \\
\text { decisiones en el momento en que se crea una decisión. }\end{array}$ \\
\hline
\end{tabular}


La evolución de las decisiones está registrada en la clase EvolutionDD. Añade a la decisión de diseño los atributos (tabla 4.5) que permite identificar quien modificó (modifiedBy) una decisión y la versión de una decisión de diseño (versión), entre otros.

Tabla 4.5. Atributos de la clase EvolutionDD.

\begin{tabular}{l|l}
\hline Atributo & Descripción \\
\hline identifierDD & Identificador de la decisión de diseño a la que complementa esta entidad. \\
\hline modifiedBy & Identificador del stakeholder que modifica la decisión. \\
\hline version & $\begin{array}{l}\text { Identifica una versión estable de la misma decisión. Una misma versión de } \\
\text { una DD incluye múltiples cambios en sus atributos. }\end{array}$ \\
\hline relatedPrinciples & Es la relación de principios de diseño. \\
\hline scope & Qué elementos pueden ser afectados por una decisión. \\
\hline evolutionReason & Define la causa del cambio. \\
\hline alternativeDD & Aquellas que se han considerado como opción válida a la DD tomada. \\
\hline
\end{tabular}

La clase HistoryDD contiene todas las decisiones de diseño que son obsoletas. Para garantizar una gestión sostenible de esta decisión. Estos atributos son un subconjunto de los atributos utilizados por el Stakeholder para capturar una decisión de diseño, e incluye el estado de la decisión (status), la categoría (category), quien (createdBy) y cuando (createdWhen) la creo, quien (modifiedBy) y cuando (modifiedWhen) la modificó, el identificador (version) de versión y la relación (relatedOn) con otras decisiones. Los detalles de estos atributos están descritos en la tabla 4.6. 
Tabla 4.6. Atributos propuestos para la clase HistoryDD.

\begin{tabular}{|c|c|}
\hline Atributo & Descripción \\
\hline identifierHistoryDD & $\begin{array}{l}\text { Identificador de la decisión de diseño en el conjunto histórico de las } \\
\text { decisiones de diseño. }\end{array}$ \\
\hline identifierDD & $\begin{array}{l}\text { Identificador de la decisión de diseño a la que complementa esta } \\
\text { entidad. }\end{array}$ \\
\hline name & Contiene el nombre de la decisión. \\
\hline status & $\begin{array}{l}\text { Determina el estado de la decisión en el momento de ser registrada en el } \\
\text { histórico. Se corresponde con aceptada (pending), aprobada (accepted), } \\
\text { rechazada (rejected). }\end{array}$ \\
\hline category & $\begin{array}{l}\text { Se corresponde a la clasificación otorgada a cada decisión en el } \\
\text { momento de ser registrada en el histórico, y relacionada a si es la } \\
\text { decisión principal (main), derivada de una principal (derived) o } \\
\text { alternativa a una principal (alternative). }\end{array}$ \\
\hline createdBy & Identificador del arquitecto que toma la decisión. \\
\hline createdWhen & Fecha en la que se crea la decisión. \\
\hline modifiedBy & Identificador del stakeholder que modifica la decisión. \\
\hline modifiedWhen & Fechas en las que se han modificado la decisión. \\
\hline version & $\begin{array}{l}\text { Identifica una versión estable de la misma decisión. Una misma versión } \\
\text { de decisión de diseño puede haber sufrido múltiples cambios en sus } \\
\text { atributos. }\end{array}$ \\
\hline relatedOn & $\begin{array}{l}\text { Permite conocer las relaciones de esta decisión con el resto de } \\
\text { decisiones. }\end{array}$ \\
\hline
\end{tabular}

Finalmente proponemos el paquete Sustainability of the Design Decision Model (figura 4.5) que nos permite medir la sostenibilidad de una arquitectura y sus soluciones en base a diferentes aspectos de calidad. Entre estos aspectos sugerimos medir la cantidad de conocimiento a capturar, el impacto cuando las decisiones cambian y determinados "architectural smells" como puede ser la complejidad de una red de decisiones. Finalmente, incorporamos los componentes externos Technical Debt Metrics, para estimar deficiencias arquitectónicas e Impact Analisys Methods como base para nuestra propuesta de análisis de impacto. 


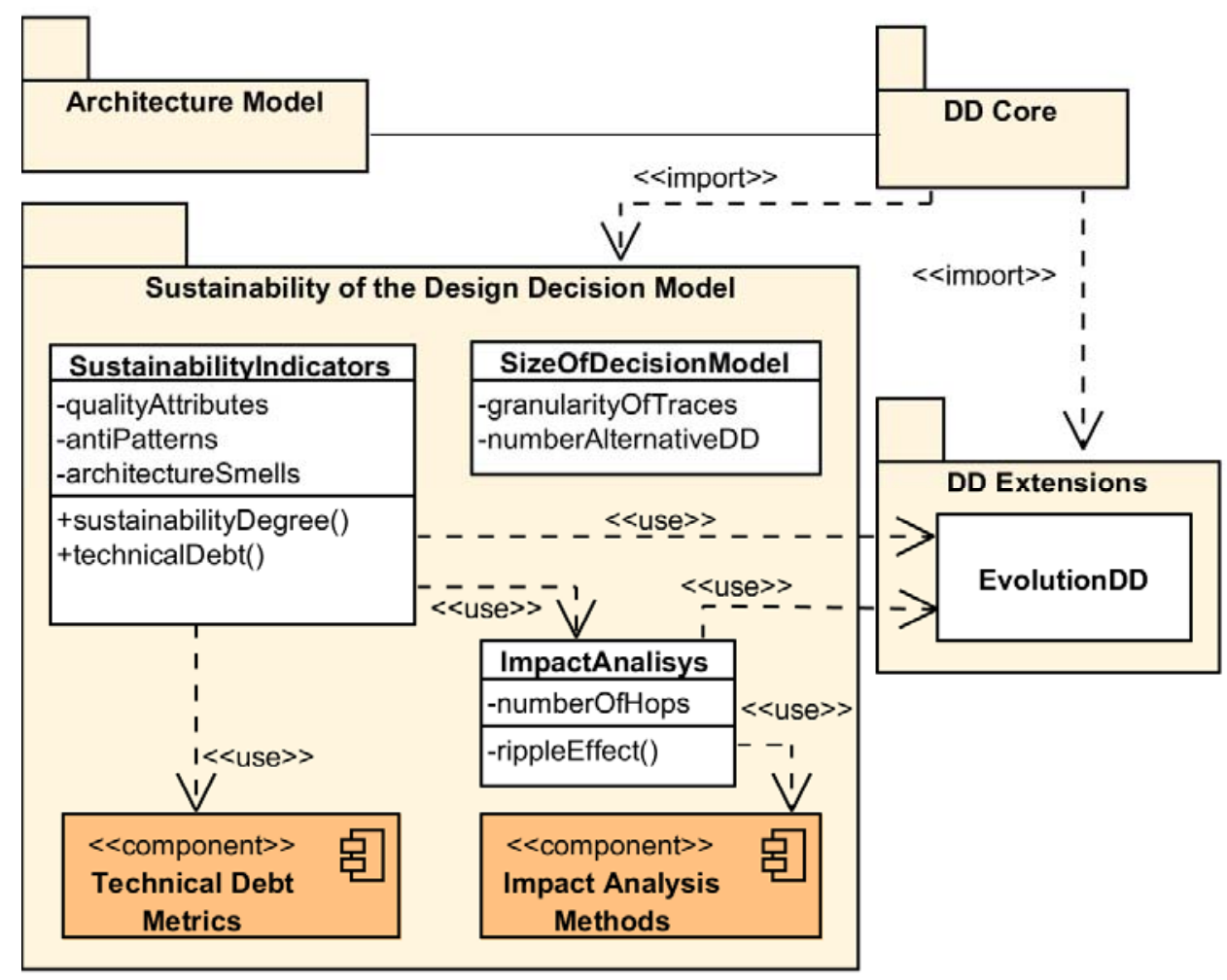

Figura 4.5: Paquete "Sustainability of the Design Decision Model".

Proponemos la clase ImpactAnalysis para sugerir estimaciones como resultado del algoritmo ripple effect cuando las decisiones cambian. Por otra parte, la clase SustainabilityIndicator sugiere métricas o combinaciones de ellas para medir cómo de sostenible es un conjunto de decisiones y que permitan mejorar determinados aspectos de calidad.

Una vez presentado nuestra propuesta de metamodelo para el conocimiento arquitectónico sostenible, vamos a describir nuestra propuesta de criterios para tener un conocimiento arquitectónico sostenible.

\subsection{Criterios para un Conocimiento Arquitectónico Sostenible}

Con el fin de resolver a uno de los problemas planteados en el capítulo 3, en este apartado definimos un conjunto de criterios que nos van a guiar para determinar como de sostenible debe ser un conjunto de decisiones de diseño. 


\subsubsection{Sostenibilidad del conocimiento arquitectónico}

En este apartado describiremos los criterios que proponemos para el mantenimiento sostenible del conocimiento arquitectónico, con el objetivo de reducir el impacto de un cambio en una decisión de diseño en función del tamaño del modelo de decisiones y granularidad, sus atributos y del número de relaciones de trazabilidad entre los diferentes elementos arquitectónicos, siendo éstos los siguientes:

C1. Granularidad de las decisiones de diseño y relaciones de trazabilidad. En la sección 4.1 de este trabajo establecimos como hipótesis de trabajo que el nivel más fino de granularidad de las decisiones de diseño se corresponde con el nivel de clase de diseño.

Argumentación: Proponemos limitar la granularidad de las decisiones de diseño a nivel de clases y paquetes y la trazabilidad entre decisiones se establece entre estos elementos de diseño. Con esta propuesta evitamos la captura de decisiones de diseño que afectan a atributos y métodos y que implicaría un número excesivo de decisiones de menor relevancia que pueden sobrecargar el número de decisiones capturadas. Además, conseguimos limitar el número de relaciones y hacer una gestión del conocimiento más sostenible. Esto no quiere decir que en algún momento el arquitecto software no pueda capturar decisiones de diseño con otro nivel de granularidad. Por ello reducimos la complejidad (Complexity) de la captura de las decisiones de diseño y aumentamos la estabilidad (Stability), porque la existencia de cambios en los atributos no implica necesariamente un nuevo elemento de representación del conocimiento.

Relación con el meta-modelo: Podemos configurar este criterio mediante el atributo granularityOfTraces de la clase SizeOfDecisionModel y afecta principalmente a Architecture Model, DD Core y DD Extensions del meta-modelo propuesto en la sección 4.2.1.

C2. Limitar el tamaño del espacio de decisiones de diseño. Los arquitectos software involucrados en el proceso de creación de una arquitectura software llegan a considerar un elevado número de decisiones alternativas que dificulta la gestión del conocimiento arquitectónico. 
Argumentación: Reduciendo el número de decisiones de diseño alternativas a considerar conseguimos que el modelo de decisiones de diseño sea más manejable, con un menor coste o esfuerzo (cost) en la captura de las decisiones de diseño y por lo tanto más sostenible.

Relación con el meta-modelo: Este criterio está representado en nuestro metamodelo por el atributo numberAltenativeDD, perteneciente a SizeOfDecisionModel, y afecta a las clases DesignDecision, Rationale, EvolutionDD y LongevityDD (figura 4.3).

C3. Limitar el número de atributos en la captura de decisiones de diseño. Las decisiones de diseño están caracterizadas por sus atributos obligatorios y opcionales (sección 2.2.2 de este trabajo). A medida que el número de decisiones crece, también crece el número de atributos de manera no lineal, dificultando la gestión del conocimiento arquitectónico.

Argumentación: Reduciendo el número de atributos a capturar, controlamos el tamaño del conocimiento arquitectónico a gestionar y reducimos el esfuerzo (cost) necesario para capturar las decisiones de diseño. Cada organización será responsable de determinar que atributos son necesarios para capturar las decisiones de diseño.

Relación con el meta-modelo: En el paquete DD Core (figura 4.3) de nuestro metamodelo están ubicados los atributos utilizados para representar estas decisiones y en el paquete DD Extensions, concretamente las clases EvolutionDD, ExtendedRationale y LongevityDD, (figura 4.4) podemos capturar el resto de atributos opcionales que podrán ser adaptado a las necesidades de cada organización para capturar más conocimiento.

\subsubsection{Sostenibilidad en la evolución del conocimiento arquitectónico}

A continuación, describimos nuestra propuesta para hacer más sostenible la evolución del conocimiento adquirido. Estos criterios deberán tener cuenta factores como el número de decisiones afectadas por el cambio de una de ellas, el número de veces que una decisión cambia o cada cuanto tiempo es necesario revisar el conjunto de decisiones para garantizar la estabilidad del conocimiento arquitectónico.

C4. Limitar el número decisiones impactadas por un cambio: No presuponemos un límite máximo de decisiones impactadas, sino que trataremos de sugerir cuales de ellas deberán ser revisadas. 
Argumentación: Con este criterio evitamos evaluar decisiones cuyo impacto visible se encuentre muy alejado del cambio principal. Sin embargo, somos conscientes que siempre pueden existir decisiones impactadas importantes y que estén fuera del alcance de esta limitación. Con este criterio reducimos la dificultad de analizar cómo afectan ciertos cambios (Changeability) en el conjunto de decisiones al limitar el número de decisiones afectadas y analizadas. Además, aumentamos la estabilidad (Stability) porque reducimos el número de cambios en el tiempo.

Relación con el meta-modelo: Esta limitación se refleja en nuestro meta-modelo en el atributo numberOfHops de la clase ImpactAnalisys situado en el Sustainability of the design decision Model (figura 4.5).

\section{C5. Supervisar el número de cambios que se producen en una decisión de diseño.} Es necesario controlar cuantas veces cambia una decisión.

Argumentación: Al aplicar este criterio, controlamos el número de veces que una decisión cambia y cuantas veces ese cambio afecta a las decisiones existentes. Además, acotamos la posibilidad de pérdida del conocimiento por los cambios existentes y aumentamos la estabilidad (Stability) del conjunto de decisiones de diseño.

Relación con el meta-modelo: Proponemos para ellos utilizar el atributo numberOfChanges de la clase LongevityDD del paquete $D D$ Extensions del meta-modelo (figura 4.4) para señalar un umbral de número de cambios, que una vez superado, solicitará la intervención del arquitecto para analizar la estabilidad de la arquitectura. Además, es importante también analizar la amplitud del intervalo de tiempo en el que se producen estos cambios. Este intervalo de tiempo está representado por los atributos createdWhen y modifiedWhen ambos en la misma clase LongevityDD.

C6. Supervisar la validez de las decisiones de diseño. Es necesario verificar que no existen decisiones obsoletas para reducir la erosión en el conocimiento arquitectónico.

Argumentación: Proponemos que el arquitecto software revise la validez (Timeless) de las decisiones para garantizar la estabilidad (Stability) de la arquitectura. La estabilidad y la validez de las decisiones afecta por tanto a su longevidad y ayudan a identificar aquellas que perduran en el tiempo como un indicador de la sostenibilidad. 
Relación con el meta-modelo: El atributo validity de la clase LongevityDD de nuestro meta-modelo (figura 4.5) determina la fecha en la que el arquitecto software debe revisar la decisión asociada.

\subsubsection{Taxonomía para estimar la sostenibilidad del conocimiento arquitectónico}

Los criterios para cuantificar el conocimiento sostenible, indicados en las secciones anteriores, forman parte de nuestra propuesta de taxonomía para evaluar la sostenibilidad del conocimiento arquitectónico.

Esta taxonomía ha sido presentada por el autor de este trabajo en [CAPI 17] y la mostramos en las tablas 4.7 y 4.8. En nuestra propuesta relacionamos los criterios ya indicados anteriormente con aquellos atributos de calidad que consideramos que están implicados y métricas involucradas en su cuantificación. El conjunto de atributos de calidad referenciado en nuestra taxonomía no es excluyente, pero consideramos que el atributo indicado es el principal atributo de calidad implicado de manera positiva en cada criterio.

Además, proponemos el uso de métricas para evaluar cada criterio, a pesar que consideramos que para evaluar adecuadamente la sostenibilidad se requiere cuantificar diferentes elementos arquitectónicos utilizando para ello diversas métricas, como por ejemplo número de decisiones (NodeCount), de relaciones de trazabilidad (EdgeCount), incluso de decisiones de diseño dependientes (number of Children). En evolución del conocimiento (tabla 4.8) se pueden combinar métricas de análisis de cambios. 
Tabla 4.7 Criterios y métricas para medir la sostenibilidad del conocimiento arquitectónico en al mantenimiento .

\begin{tabular}{|c|c|c|c|}
\hline $\begin{array}{l}\text { Gestión del } \\
\text { conocimiento } \\
\text { arquitectónico }\end{array}$ & $\begin{array}{c}\text { Criterios para la } \\
\text { sostenibilidad del } \\
\text { conocimiento arquitectónico }\end{array}$ & $\begin{array}{l}\text { Atributos de } \\
\text { calidad }\end{array}$ & Métricas \\
\hline \multirow{4}{*}{$\begin{array}{l}\text { Mantenimiento del } \\
\text { conocimiento } \\
\text { arquitectónico } \\
\text { Reducir el conjunto de } \\
\text { decisiones de diseño y } \\
\text { controlar el número de } \\
\text { vínculos de rastreo } \\
\text { entre las decisiones y } \\
\text { otros artefactos de } \\
\text { software facilita el } \\
\text { mantenimiento } \\
\text { conocimiento de } \\
\text { arquitectónico cuando } \\
\text { las decisiones cambian. }\end{array}$} & \multirow{2}{*}{$\begin{array}{l}\text { C1. Granularidad de las } \\
\text { decisiones de diseño y } \\
\text { relaciones de traza. Limitar la } \\
\text { granularidad de las decisiones a } \\
\text { capturar en paquetes y clases, } \\
\text { evita decisiones más finas y } \\
\text { reduce el tamaño del modelo de } \\
\text { decisión. Por ejemplo, las } \\
\text { decisiones que implican la } \\
\text { creación de clases UML serán } \\
\text { capturadas, pero las decisiones } \\
\text { relativas a los atributos UML o } \\
\text { métodos no lo serán. Evitar } \\
\text { decisiones finas reduce el } \\
\text { número de enlaces de traza a } \\
\text { otros artefactos de software. }\end{array}$} & $\begin{array}{lr}\text { Complexity. } & \text { La } \\
\text { complejidad de una } \\
\text { red de decisiones } \\
\text { puede ser reducida si } \\
\text { limitamos } & \text { la } \\
\text { granularidad de las } \\
\text { decisiones } & \\
\text { capturadas y el } \\
\text { número de trazas } \\
\text { entre ellas }\end{array}$ & $\begin{array}{l}\text { NodeCount } \\
\text { EdgeCount }\end{array}$ \\
\hline & & $\begin{array}{lr}\text { Stability. } & \text { Los } \\
\text { cambios en } & \text { un } \\
\text { elemento } & \text { del } \\
\text { conocimiento no } \\
\text { afecta a las } \\
\text { decisiones } \\
\text { capturadas, al no ser } \\
\text { necesario añadir un } \\
\text { nuevo nodo en la red } \\
\text { de decisiones. }\end{array}$ & Instability \\
\hline & $\begin{array}{l}\text { C2. Tamaño del modelo de } \\
\text { decisión. El número de } \\
\text { alternativas de diseño debe ser } \\
\text { limitado. Nuestra experiencia en } \\
\text { diferentes proyectos sugiere } \\
\text { mantener cada decisión para 1-8 } \\
\text { opciones alternativas y, si las } \\
\text { decisiones se vuelven más } \\
\text { complejas, modelar partes de } \\
\text { ellas como decisiones de } \\
\text { seguimiento. }\end{array}$ & $\begin{array}{l}\text { Coste del esfuerzo } \\
\text { para capturar pocas } \\
\text { decisiones. }\end{array}$ & $\begin{array}{l}\text { Number } \\
\text { Children }\end{array}$ \\
\hline & $\begin{array}{l}\text { C3. Número de atributos de } \\
\text { elementos arquitectónico } \\
\text { capturados. Capturar menos } \\
\text { elementos del conocimiento } \\
\text { arquitectónico (entre tres y seis) } \\
\text { utilizando plantillas } \\
\text { configurables hace que dicho } \\
\text { conocimiento sea más } \\
\text { manejable. }\end{array}$ & $\begin{array}{l}\text { Coste del esfuerzo } \\
\text { para capturar varios } \\
\text { atributos variables } \\
\text { del conocimiento. }\end{array}$ & $\begin{array}{l}\text { Number } \\
\text { Fields }\end{array}$ \\
\hline
\end{tabular}


Tabla 4.8. Criterios y métricas para medir la sostenibilidad del conocimiento arquitectónico en a la evolución.

\begin{tabular}{|c|c|c|c|}
\hline $\begin{array}{l}\text { Gestión del } \\
\text { conocimiento } \\
\text { arquitectónico }\end{array}$ & $\begin{array}{c}\text { Criterios para la } \\
\text { sostenibilidad del } \\
\text { conocimiento arquitectónico }\end{array}$ & Atributos de calidad & Métricas \\
\hline \multirow[t]{3}{*}{$\begin{array}{l}\text { Evolución. } \\
\text { Una mejor estimación y } \\
\text { limitación del número } \\
\text { de decisiones } \\
\text { impactadas por un } \\
\text { cambio ayuda a reducir } \\
\text { el número de decisiones } \\
\text { a analizar durante los } \\
\text { ciclos de evolución. } \\
\text { Además, saber con } \\
\text { anticipación } \\
\text { decisiones deben ser } \\
\text { revisadas permite una } \\
\text { mejor predicción de la } \\
\text { estabilidad rad } \\
\text { longevidad del modelo } \\
\text { AK. }\end{array}$} & $\begin{array}{l}\text { C4. Número de decisiones de } \\
\text { diseño afectadas. } \\
\text { Las decisiones que cambian } \\
\text { afectan a otras decisiones } \\
\text { relacionadas. Por lo tanto, } \\
\text { limitar este impacto, } \\
\text { utilizando un algoritmo de } \\
\text { análisis de impacto (ripple } \\
\text { effect) puede reducir el } \\
\text { número de decisiones que } \\
\text { deben ser analizadas o } \\
\text { revisadas. Los diseñadores } \\
\text { deben establecer este límite } \\
\text { sobre su propia experiencia. } \\
\text { Aquellas decisiones de red que } \\
\text { son "nodos finales" también } \\
\text { pueden ser limitadas. }\end{array}$ & 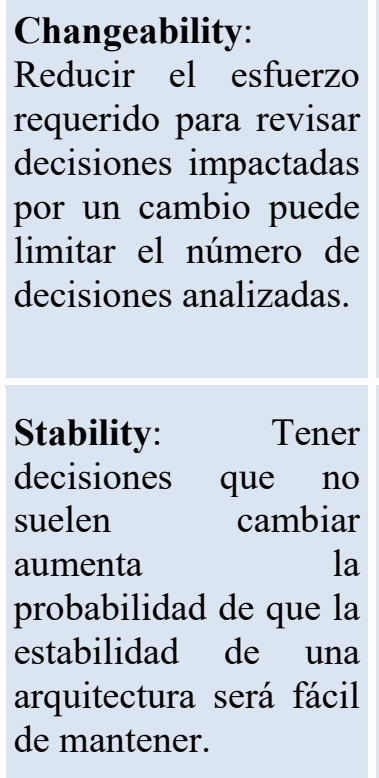 & $\begin{array}{l}\text { Change } \\
\text { Impact } \\
\text { Analysis } \\
\text { (ripple effect) } \\
\text { Change } \\
\text { proneness }\end{array}$ \\
\hline & $\begin{array}{l}\text { C5. Número de veces que } \\
\text { una decisión cambia. La } \\
\text { información sobre cuántas } \\
\text { veces una decisión cambia } \\
\text { durante un período específico } \\
\text { puede ser rastreada y usada } \\
\text { para controlar la estabilidad de } \\
\text { la decisión, ayudando a indicar } \\
\text { la longevidad de las decisiones } \\
\text { más estables. }\end{array}$ & 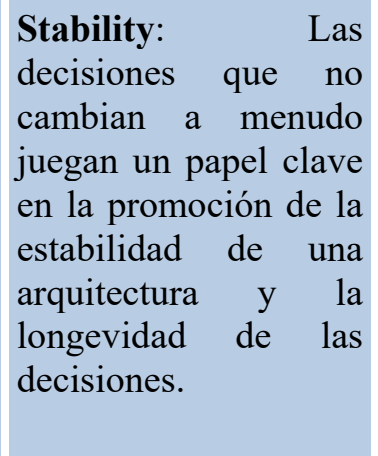 & $\begin{array}{l}\text { Decision } \\
\text { Volatility }\end{array}$ \\
\hline & $\begin{array}{l}\text { C6. Validez de las decisiones. } \\
\text { Pueden establecerse fechas } \\
\text { específicas para la revisión de } \\
\text { la decisión y se pueden } \\
\text { eliminar las decisiones } \\
\text { obsoletas. }\end{array}$ & Stability, Timeliness & $\begin{array}{l}\text { No está } \\
\text { definida. } \\
\text { Uso de la } \\
\text { marca } \\
\text { timestamp en } \\
\text { las decisiones. }\end{array}$ \\
\hline
\end{tabular}




\subsection{Cálculo de la Sostenibilidad}

A lo largo de esta sección vamos a proponer métricas para evaluar la sostenibilidad del conocimiento arquitectónico centrado en las decisiones de diseño, aplicando los criterios de sostenibilidad propuestos en la sección 4.2 de este trabajo y tomando como referencia los atributos de calidad relacionados con estos criterios (tabla 4.7 y tabla 4.8). Posteriormente, en el capítulo de experimentación procederemos a validar estas propuestas de métricas.

\subsubsection{Sostenibilidad basada en la complejidad}

A continuación, proponemos una métrica para evaluar la sostenibilidad en la gestión del conocimiento, centrada en la complejidad que presenta el conjunto de decisiones y relaciones de trazabilidad ya capturadas. Esta evaluación es el resultado de aplicar el criterio “C1. Granularidad de las decisiones de diseño y relaciones de trazabilidad” presentado en la sección 4.3.1 y también en nuestra taxonomía (tabla 4.7).

Como hemos indicado en el capítulo 4 de este trabajo, nuestro punto de partida es que la red de decisiones de diseño la representaremos como un grafo dirigido. En general, el arquitecto sigue este sentido durante el proceso de diseño arquitectónico, porque toma una decisión a partir de otra ya existen, estableciendo una relación de dependencia entre decisiones similar al modelo padre-hijo.

Los elementos arquitectónicos a tener en cuenta por nuestra métrica son el número de decisiones y de trazas. Distinguiremos varios tipos de decisiones, como son aisladas, no aisladas sin otras decisiones dependientes (sin hijos) y no aisladas con otras decisiones dependientes (con hijos), porque cada una de ellas influyen de distinta manera en nuestra propuesta. Consideramos que el tipo y número de las relaciones de trazabilidad que mantienen con otras decisiones influyen en la complejidad del conocimiento arquitectónico. Nuestra propuesta la detallamos en la ecuación 4.1:

$$
\text { Complexity }=\frac{\left(\sum_{i=0}^{i s o D D} \alpha_{D D i}\right)+\text { numIsoOutChDD }+\left(\sum_{i=0}^{\text {numIsoInChDD }} \text { childrenNumber }_{i}\right)}{T_{T}+D D_{T}}
$$

Ecuación 4.1 Complejidad de una red de decisiones 
Los diferentes términos de la ecuación son:

$\alpha_{D D i}=$ Factor de corrección de importancia configurable para decisión aislada i_esima . isoDD = Número de decisiones aisladas.

numIsoOutChDD = Número de decisiones no aisladas que no tienen hijos.

$\sum_{i=0}^{\text {numIsoInChDD }}$ childrenNumber ${ }_{i}=$ num. de decisiones que dependen de cada una no aislada.

$T_{T}=$ Número total de trazas de la red de decisiones.

$D D_{T}=$ Número total de decisiones de la red de decisiones.

Cada término del numerador representa un tipo de decisión según lo expuesto anteriormente. El primer término, $\sum_{i=0}^{i s o D D} \alpha_{D D i}$, representa como afecta el subconjunto de decisiones aisladas cuando aplicamos el criterio C1. En base a la experimentación de capturar decisiones, la gestión de una decisión aislada no es igual de compleja que la de una decisión con relaciones con otras. Sencillamente porque no es posible gestionar su relación de dependencia y porque un cambio no afecta al resto de decisiones. Por ello, proponemos aplicar el factor de corrección configurable $\alpha_{D D i}$ en el intervalo $[0,1] \mathrm{y}$ personalizado para cada decisión aislada.

Consideramos que cada decisión aislada puede tener una complejidad distinta (p.e. distinto número de atributos), por influir de distinta manera a la complejidad del conjunto de decisiones. Por ello, proponemos que cada decisión aislada tenga un factor $\alpha_{D D i}$, con valores pertenencientes a [0...1], que indica cómo de importante una decisión aislada para el arquitecto. Si $\alpha_{D D i}$ es 1, el arquitecto considera que la decisión aislada presenta una gestión igual de compleja que la presentada por una no aislada. Y si $\alpha_{D D i}$ es 0 , se considera que esa decisión no introduce complejidad.

Los siguientes términos del numerador representan a las decisiones no aisladas, y diferenciamos las que no tienen dependientes (numIsoOutChDD) y de las que sí tienen (numIsoInChDD). En este caso, la complejidad que presenta las decisiones con hijos es proporcional al número de decisiones que dependen de dicha decisión, porque un cambio en una decisión no aislada puede afectar a sus decisiones dependientes. En cualquier caso, tengan o no decisiones hijos, dicho cambio requiere verificar que no está en contradicción con la decisión predecesora. En el denominador de nuestra ecuación propuesta, está representado la composición del conocimiento como el conjunto de decisiones y de relaciones, $T_{T}+D D_{T}$. De esta manera ponderamos la complejidad de una red en función 
de su propia topología y también poder comparar la complejidad de dos redes en función de su composición.

Justificación: Los valores de complejidad que obtenemos con nuestra métrica de complejidad se encuentran normalizados en el intervalo [0...1] ya que dividimos el valor de la complejidad por el número total de decisiones más el número total de relaciones de trazabilidad. El numerador va a ser siempre menor o igual que el denominador ya que si tuviéramos decisiones aisladas van a tener una importancia relativa menor que 1 , y si no existen decisiones aisladas, el número de decisiones con hijos y sin hijos va a ser siempre menor que el del denominador.

Para computar el número de decisiones aisladas, isoDD, no aisladas sin hijos, numIsoOutChDD, y no aisladas con hijos, numIsoInChDD, tendremos en cuenta algoritmos para contar el número de nodos, NodeCount y de aristas EdgeCount. A esta métrica la denominamos Architectural Design Decision Network Complexity (ADDN-C) al cuantificar la complejidad del conocimiento, y que estará incluida en la aplicación Architectural Design Decision Network Metrics (ADDN-Metrics) cuyo diseño UML está detallado en el Anexo III y su implementación reflejado en el Anexo IV de este trabajo.

Cambios en el metamodelo: Esta propuesta de métrica está reflejada en el metamodelo de la figura 4.8 de la sección 4.4.4. En dicha sección analizaremos en profundidad los elementos representados en dicho metamodelo ya que en él también reflejamos los elementos que intervienen en otras propuestas de solución que forman parte de este trabajo.

Contribución: A diferencia de las métricas de complejidad orientada a código como son las de McCabe ${ }^{6}$ [MCCA 76], Chidamber ${ }^{7,8}$ [CHID 94] [KAN 02], [KOZI 11], [FENT 14] y de métricas de complejidad orientadas a la arquitecturas software y basadas en la estimación de la complejidad de los módulos, nuestra propuesta se enfoca de manera exclusivamente a la estimación de la complejidad en redes de decisiones.

\footnotetext{
${ }^{6}$ La métrica de complejidad ciclomática de McCabe, que mide el número de caminos independientes de un grafo (entendiendo este como un programa que contienen caminos que se bifuracan) no es posible adaptarla a las distintas topologías de redes de decisiones actuales.

${ }^{7}$ Coupling between object classes (CBO). Calcula el número de acoplamientos (relaciones de uso, etc.) de que una clase tiene con el resto de clases

${ }^{8}$ Response for class (RFC). Mide el número de llamadas internas y externas a un método de una clase.
} 


\subsubsection{Sostenibilidad basada en el esfuerzo de capturar decisiones}

A continuación, proponemos una métrica para evaluar la sostenibilidad en la gestión del conocimiento, centrada en el esfuerzo requerido para capturar decisiones de diseño. Esta evaluación es el resultado de aplicar el criterio “C3. Limitar el número de atributos en la captura de decisiones de diseño" presentado en la sección 4.3.1 y también en nuestra taxonomía (tabla 4.7).

Consideramos que a mayor número de decisiones a capturar mayor debe ser el esfuerzo de capturarlas. Además, el número de atributos que forman parte de cada decisión también influye, de tal manera que a mayor número de atributos mayor esfuerzo. Por ello, nuestra propuesta de métrica de esfuerzo será proporcional al número de decisiones a capturar y al número de atributos que forman cada decisión.

Otro factor a tener en cuenta en nuestra propuesta es el esfuerzo el arquitecto en capturar decisiones. Este esfuerzo se mide en el tiempo requerido en capturar las decisiones. Además, este esfuerzo incluye el tiempo de pensar, capturar y de modificar, si es necesario, cada decisión.

Por lo tanto, el esfuerzo requerido para capturar decisiones por un arquitecto está en función del número de decisiones que captura $\left(\right.$ num $\left._{D D}\right)$, del número de atributos $\left(\right.$ num $\left._{\text {Atributos }}\right)$ y del tiempo requerido en horas $t_{\text {horas }}$, tal y como mostramos en la ecuación 4.2.

$$
\text { Esfuerzo }_{\text {arquitecto }}=\frac{\text { num }_{\text {Atributos }} * \text { num }_{D D}}{t_{\text {horas }}}
$$

Ecuación 4.2: Esfuerzo de un arquitecto en capturar decisiones.

Cuando las decisiones son tomadas por un grupo de arquitectos y es posible cuantificar el esfuerzo de cada arquitecto, el esfuerzo total será la suma de esfuerzos de cada arquitecto, tal y como proponemos en la ecuación 4.3. A esta métrica propuesta la llamaremos Architectural Design Decision Network Effort (ADDN-E).

$$
\text { Esfuerzo }_{\text {Total }}=\sum_{i=0}^{n} \text { Esfuerzo }_{\text {arquitecto }_{i}}
$$

Ecuación 4.3: Esfuerzo total en la captura de decisiones. 
Cuando la captura de decisiones es tomada por un grupo de arquitectos, y no es posible estimar el esfuerzo individual de cada arquitecto, proponemos calcular el esfuerzo total considerando que num $_{D D}$ es el número total de decisiones capturadas por el grupo y $t_{\text {horas }}$ el número de horas empleadas en capturar dichas decisiones por todo el grupo de arquitectos.

Cambios en el metamodelo: Esta propuesta de métrica está basada en el modelo de la figura 4.8 de la sección 4.4.4, que la analizaremos más adelante.

De manera complementaria para la estimación del esfuerzo, proponemos tres tipos de plantillas para capturar 7, 10 y 14 atributos, de tal manera que el arquitecto puede elegir una de ellas para capturar las decisiones de diseño. En la tabla 4.9 mostramos todos los atributos

Tabla 4.9. Plantilla de atributos a capturar

\begin{tabular}{|c|c|c|c|c|}
\hline System: SCE & ID: & Identificador de la decisión & Date: & Fecha \\
\hline Name & \multicolumn{4}{|c|}{ Contiene el nombre de la decisión. } \\
\hline Description & \multicolumn{4}{|c|}{ Contiene la descripción de la decisión. } \\
\hline Rationale & \multicolumn{4}{|c|}{ Describe los motivos o razones que justifican una decisión. } \\
\hline Pros & \multicolumn{4}{|c|}{$\begin{array}{l}\text { Se corresponde con la evaluación favorable frente a las decisiones } \\
\text { alternativas. }\end{array}$} \\
\hline Cons & \multicolumn{4}{|c|}{$\begin{array}{l}\text { Se corresponde con la evaluación negativa frente a las decisiones } \\
\text { alternativas. }\end{array}$} \\
\hline Status & \multicolumn{4}{|c|}{$\begin{array}{l}\text { Determina el estado actual de cada decisión. Se corresponde con } \\
\text { aceptada (pending), aprobada (accept), rechazada (rejected) }\end{array}$} \\
\hline Responsible & \multicolumn{4}{|c|}{ Identifica al arquitecto que toma la decisión. } \\
\hline Alternative decisions & \multicolumn{4}{|c|}{ Identifica a las decisiones alternativas } \\
\hline $\begin{array}{l}\text { Related to } \\
\text { requirements }\end{array}$ & \multicolumn{4}{|c|}{ Relación de requisitos relacionados } \\
\hline $\begin{array}{l}\text { Related to software } \\
\text { artifacts }\end{array}$ & \multicolumn{4}{|c|}{ Relación de elementos relacionados } \\
\hline Constraints & \multicolumn{4}{|c|}{ Restricciones } \\
\hline Related to decisions & \multicolumn{4}{|c|}{ Dependencia entre decisiones } \\
\hline
\end{tabular}


En la tabla 4.10 mostramos la distribución de estos atributos en tres plantillas con 7 ("short"), 10 ("médium") y 14 ("long") atributos que proponemos para la captura de las decisiones

Tabla 4.10. Composición de las plantillas "long", "médium" y "short"

\begin{tabular}{|l|l|l|l|}
\hline Atributos & Long & Medium & Short \\
\hline ID: & & \\
\hline Date: & & \\
\hline Name & & \\
\hline Description & & \\
\hline Rationale & & \\
\hline Pros & & \\
\hline Cons & & \\
\hline Status & & \\
\hline Responsible & & \\
\hline Alternative decisions & & \\
\hline Related to requirements & & \\
\hline Related to software artifacts & & \\
\hline Constraints & & \\
\hline Related to decisions & & \\
\hline
\end{tabular}

\subsubsection{Sostenibilidad basada en la evolución}

En esta sección proponemos un mecanismo para ofrecer sostenibilidad en la evolución del conocimiento, debido a cambios que se producen en las decisiones que lo forman. Esta propuesta está basada en el criterio “C4. Limitar el número decisiones impactadas por un cambio" presentado en la sección 4.3 .2 y también en la tabla 4.8 de nuestra taxonomía. 
Nuestra propuesta se basa en calcular el conjunto de decisiones que estarán afectadas si una decisión cambia. Por ello, utilizaremos el concepto de RippleEffect para desarrollar un algoritmo adaptado que nos permita medir el efecto de los cambios en las decisiones. En primer lugar, partimos de un grafo que representa las decisiones de diseño, y en segundo lugar aplicamos una adaptación de los algoritmos clásicos de ripple effect para recorrer el grafo e identificar las decisiones relacionadas con la que cambia. Describiremos en la experimentación la creación del grafo y aquí nos centraremos en la explicación de nuestra propuesta de algoritmo del ripple effect.

Nuestro algoritmo tiene como precondiciones que el grafo este generado y que la decisión origen forma parte del mismo. Como postcondición tiene que el conjunto solución de decisiones impactadas no tenga decisiones duplicadas. El proceso de búsqueda de decisiones, partiendo de una decisión inicial, localizará todas las decisiones que se encuentren a la misma distancia, y repetiremos el proceso hasta que no haya decisiones. Nuestro algoritmo calcula por distancia al nodo origen, la lista de decisiones más cercanas, y elimina aquellas duplicadas y aquellas que se encuentran a una distancia mayor que las ya consideradas. En la figura 4.6 describimos el algoritmo ${ }^{9}$ que implementa nuestro mecanismo de ripple effect.

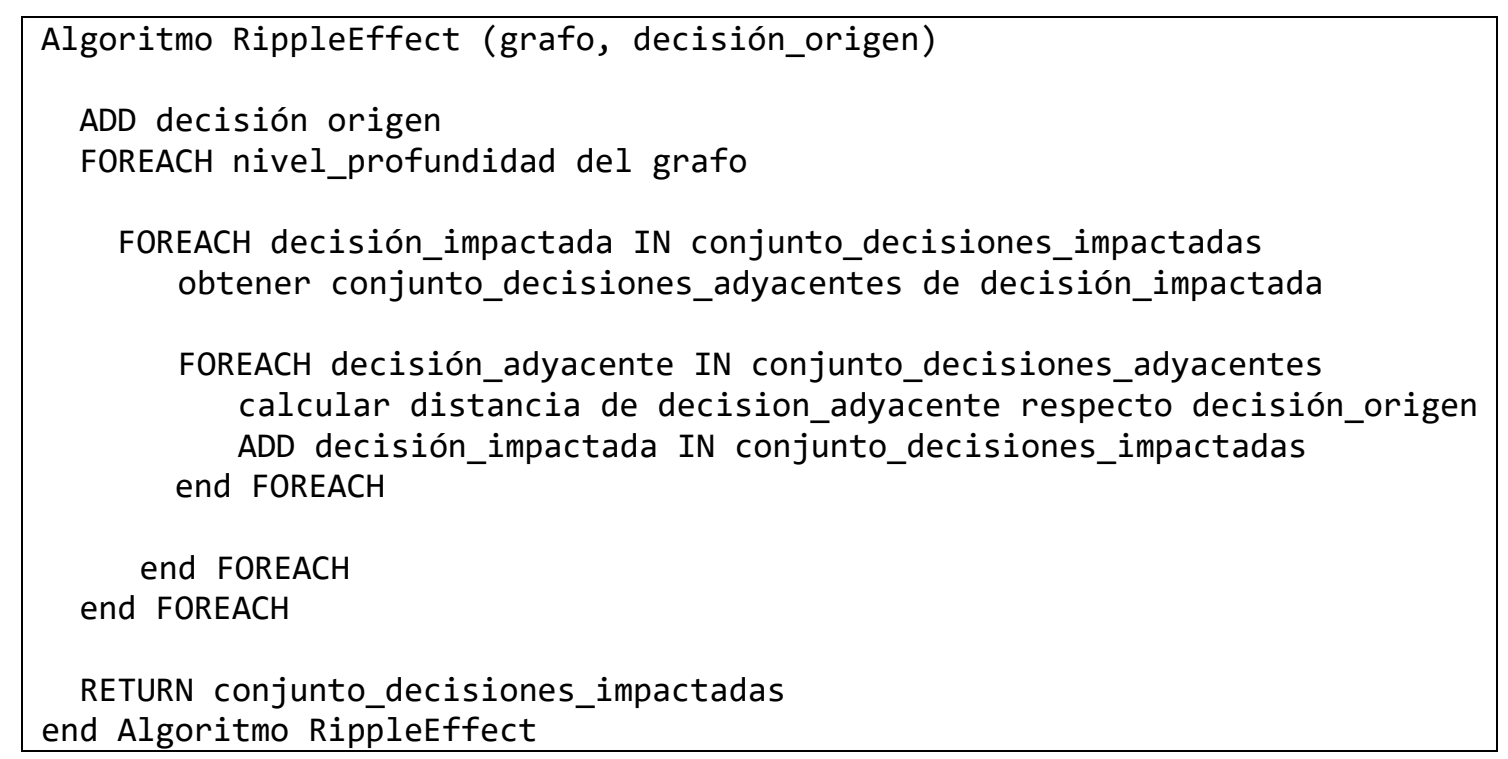
Figura 4.6: Algoritmo "RippleEffect".

\footnotetext{
${ }^{9}$ En nuestro algoritmo consideramos el uso de colecciones para poder ser recorridas mediante la sentencia de control FOREACH, sin tener que presuponer el número de elementos de cada colección.
} 
Los detalles de implementación de la figura 4.6 se indican en la figura 4.7

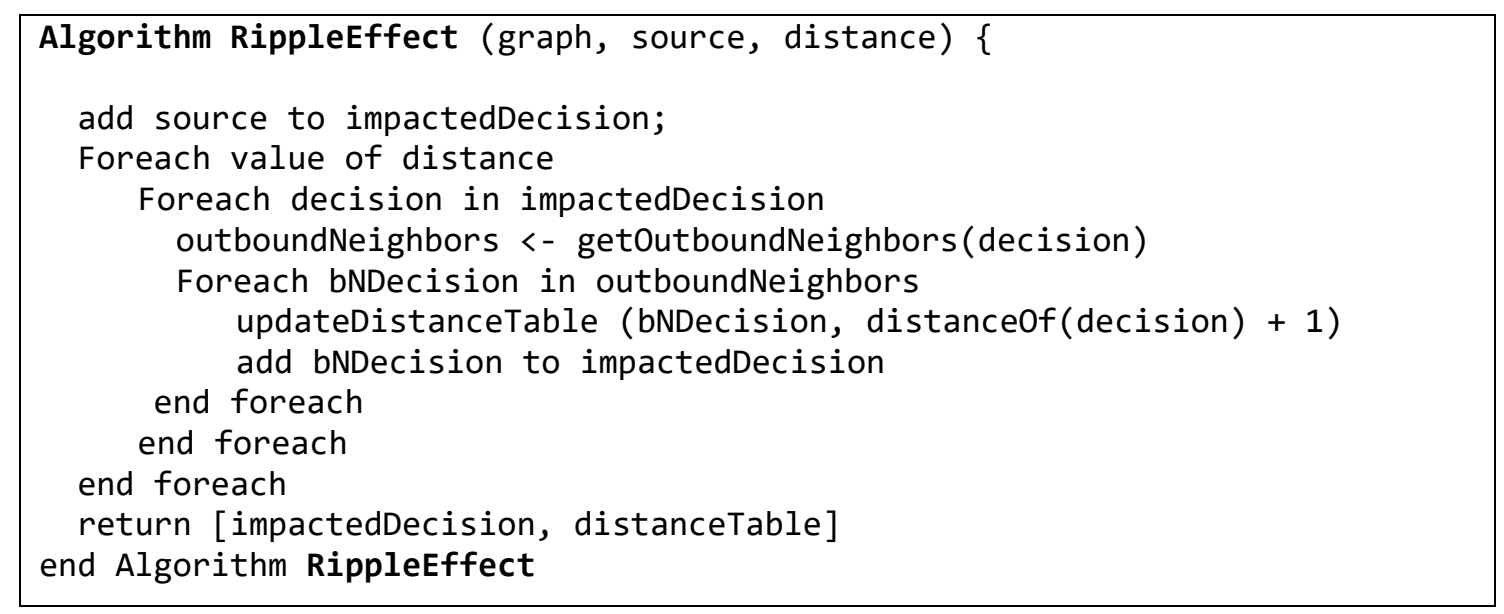

Figura 4.7: Algoritmo "RippleEffect".

Nuestro algoritmo RippleEffect utiliza las colecciones llamadas listas (inBoundNeighbors, outboundNeighbors), conjuntos (impactedDecision) y tablas hash indexadas por una decisión (distanceTable). Por lo tanto, los resultados de este algoritmo son el conjunto de decisiones obtenidos (impactedDecision) y también la tabla de distancias de decisiones respecto a la decisión origen. De esta manera, y mediante el uso combinado de estas dos colecciones, generamos los diferentes conjuntos de decisiones equidistantes al nodo origen. Este algoritmo se basa en la existencia de otros ya implementados en nuestra propuesta de métrica de complejidad $A D D N-C$, descrita anteriormente, como por ejemplo los métodos getInBoundNeighbors() y getOutboundNeighbors (), utilizados para obtener las listas de decisiones dependientes.

Evaluación cualitativa del arquitecto software: Una vez determinado el conjunto de decisiones agrupadas por cercanía a la decisión que cambia, con el fin de poder asesorar si existe una relación entre la decisión origen y el resto de decisiones, el arquitecto software deberá realizar una evaluación cualitativa comparando las decisiones encontradas con la decisión que cambia. Aquellas decisiones que no tengan relación podrán ser eliminadas del conjunto de decisiones encontradas. Si ello nos permite determinar que todas las decisiones pertenecientes a una determinada distancia " $\mathrm{x}$ " no tienen relación con la decisión origen, podríamos asesorar que el número de saltos del algoritmo ripple effect a evaluar sea todas las decisiones que se encuentren dentro del salto " $\mathrm{x}-1$ ". 
Como complemento a nuestra propuesta, proponemos combinar los resultados obtenidos por nuestro algoritmo ripple effect con una métrica de estabilidad que nos permita estimar la estabilidad de cada una de las decisiones impactadas. Con nuestra propuesta aportamos al arquitecto más elementos de valor para estimar en qué medida se verán afectadas los posibles cambios en las decisiones y determinar que decisiones son más estables.

Medida de estabilidad: Proponemos una medida para estimar la estabilidad de un conjunto de decisiones de diseño basada en la métrica [MART 94], donde estimamos los subconjuntos de decisiones más inestables como aquellos que tienen más enlaces de salida que de entrada y los más estables que cuentan con más enlaces de entrada que de salida. Por lo tanto, nuestra propuesta tendrá en cuenta el número relaciones de dependencia que una decisión tiene con sus inmediatamente adyacentes, tal y como vemos en la ecuación 4.4:

$$
\text { Instability }=\frac{\text { DDout }}{\text { DDin }+ \text { DDout }}
$$

Ecuación 4.4: Medida de estabilidad para redes de decisiones de diseño.

Donde:

DDout $=$ número de decisiones dependientes

DDin = número de decisiones de las que una decisión depende

Los valores de "instability" obtenidos estarán en el rango de [0,1], donde 0 indica que la decisión es totalmente estable y 1 indica que es totalmente inestable. Proponemos la siguiente escala de valores para determinar el grado de inestabilidad

$\left[\begin{array}{lll}0 & \ldots & 0.39\end{array}\right]$ Decisiones estables

$[0.4$... 0.6] Decisiones con grado de inestabilidad medio

[0.61 ... 1] Decisiones más inestables

A esta métrica la denominamos Architectural Design Decision Network Instability $(A D D N-I)$. Los aspectos novedosos de esta solución son: (i) aplicación de la métrica de instabilidad a grafos de decisiones de diseño y, (ii) la combinación de dos métricas para determinar cómo influyen los cambios de las decisiones en el conjunto.

Cambios en el metamodelo: Esta propuesta de métrica está basada en el modelo de la figura 4.8 de la sección 4.4.4, que analizaremos a continuación. 


\subsubsection{Cambios en el metamodelo}

En la sección 4.2.1 presentamos el meta-modelo base de nuestra propuesta. Durante el desarrollo de este trabajo hemos propuesto un modelo para incluir nuestras propuestas de criterios, métricas y mecanismos de análisis, y que lo presentamos en la figura 4.8. Por ello, a continuación vamos a indicar que elementos del modelo están relacionados con nuestras propuestas aquí presentadas. En el modelo de figura 4.8, mantenemos los paquetes Architecture Model y DD Core según lo descrito en la sección 4.2.1, no así los paquetes DD Extensions y Sustainability of the Design Decision Model ${ }^{10}$ que los hemos modificado.

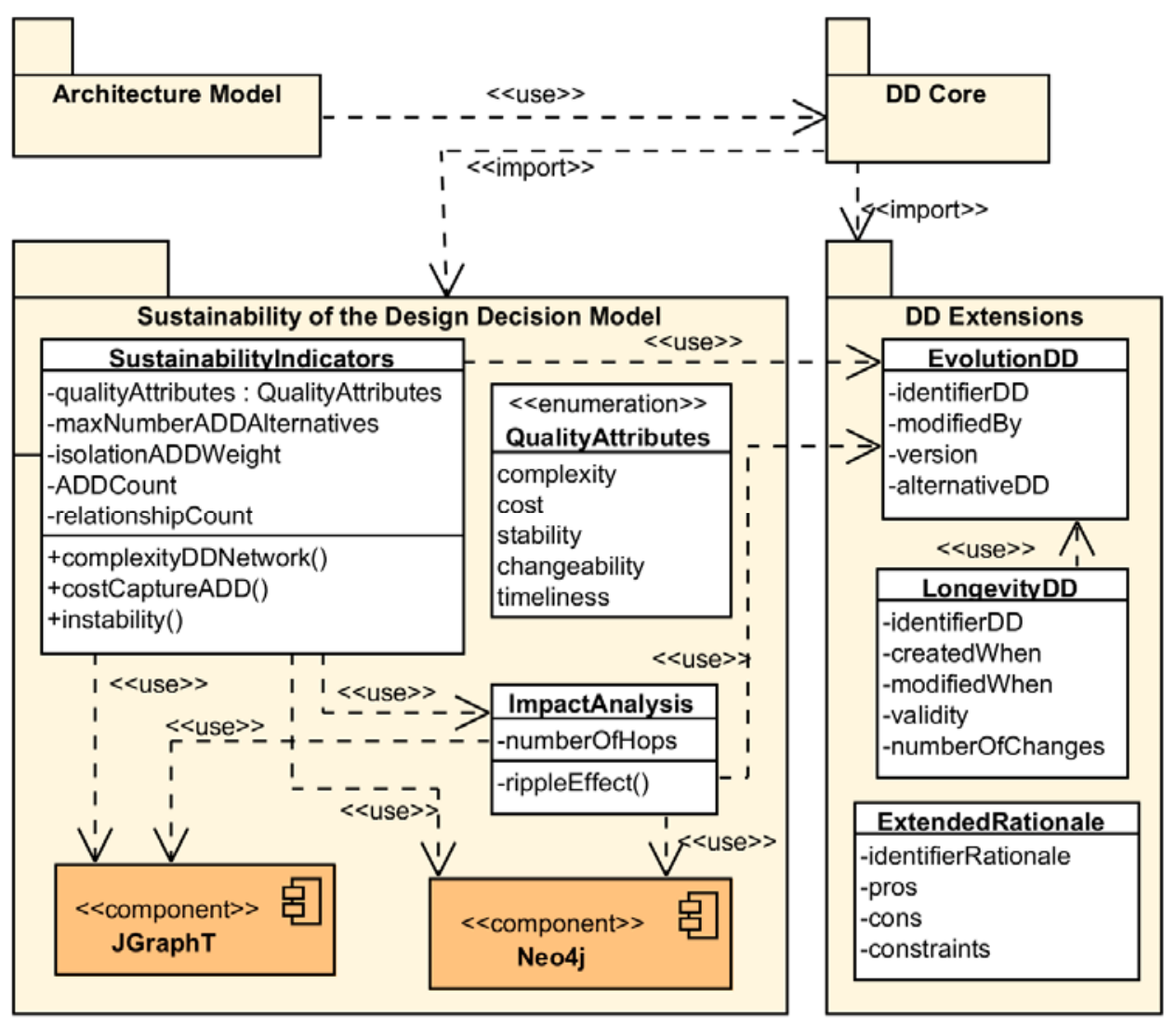

Figura 4.8: Modelo para la gestión sostenible de decisiones de diseño.

Impacto de la taxonomía: Los criterios presentados en la sección 4.3.2 señalan que su aplicación influye a un subconjunto de atributos de calidad detallados en las tablas 4.7 y 4.8. Estos atributos están enumerados en QualityAttributes del paquete Sustainability of the Design Decision Model. En la clase SustainabilityIndicators de este mismo paquete, referenciamos dichos atributos de calidad mediante qualityAttributes, y con

\footnotetext{
${ }^{10}$ Se declaran listas de valores asociados a atributos definidos en entidades de este paquete.
} 
maxNumberADDAlterntives limitamos el número de decisiones alternativas a capturar por los arquitectos.

Impacto de la métrica de complejidad: La métrica $A D D N-C$ propuesta introduce nuevos elementos en paquete Sustainability of the Design Decision Model. En base a las hipótesis del capítulo 4 de este trabajo relacionadas con la representación de la red de decisiones, hemos incorporado los componentes Neo4J ${ }^{11}$ y JGraphT ${ }^{12}$ ya que ambos componentes permiten visualizar grafos y disponen de algoritmos de grafos. En SustainabilityIndicators hemos incluido los atributos isolationADDWeight, para caracterizar el factor de corrección de importancia para una decisión aislada $\alpha_{i}$, además de ADDCount y relationshipCount para el numero de decisiones o nodos y de relaciones de traza o aristas, respectivamente. Por último, complexityDDNetwork() representa la métrica de complejidad propuesta en la sección 4.4.1.

Impacto de la métrica de esfuerzo: Para validar nuestra métrica $A D D N-E$ propuesta en la sección 4.4.2, proponemos capturar los atributos declarados en el paquete DD Core del meta-modelo de la sección 4.2.1 junto a los atributos que destacamos en el paquete DD Extensions de la figura 4.8. Además, en la clase SustainabilityIndicators incluimos $A D D C o u n t$, que representa el número de decisiones capturadas, y que interviene en costCapture $A D D()$ también incluida en esta entidad. Las clases descritas en los paquetes DD Core y DD Extensions contienen un número variable de atributos que pueden configurarse para capturar una mayor o menor cantidad de conocimiento arquitectónico.

Impacto del procedimiento de análisis de impacto en evolución: La clase ImpactAnalisys de la figura 4.8 contiene nuestro algoritmo rippleEffect para generar los conjuntos de decisiones afectadas por un cambio y el atributo numberOfHops define el nivel máximo de impacto. En cuanto a la métrica de "instability" ADDN-I propuesta, la reflejamos en el método instability() de ImpactAnalisys.

\footnotetext{
${ }^{11} \mathrm{https://neo4j.com/}$

${ }^{12}$ http://jgrapht.org/
} 


\section{Capítulo 5. Experimentación}

En este capítulo describimos como hemos realizado la experimentación de las métricas propuestas en el capítulo 4. Nuestra experimentación tiene como objetivo validar las tres métricas propuestas en la sección 4.4, teniendo como referencia marco el metamodelo descrito en la sección 4.4.4 de nuestro trabajo. Para ello, hemos realizado la experimentación en tres etapas independientes:

- Sostenibilidad basada en complejidad: Hemos validado una primera versión de la herramienta diseñada para evaluar la sostenibilidad en la red de decisiones, según la ecuación detallada en la sección 4.4.1. Esta validación la desarrollaremos en la sección 5.1 de este capítulo.

- Sostenibilidad basada en coste: Hemos realizado un experimento con un conjunto de arquitectos software para validad nuestra propuesta de métrica de coste detallada en la sección 4.4.2. Este experimento, junto al análisis de sus datos, será desarrollado en la sección 5.2.

- Sostenibilidad basada en evolución: En la sección 5.3 de este capítulo, validamos nuestra herramienta para evaluar la sostenibilidad en la evolución de la red de decisiones, según la ecuación detallada en la sección 4.4.3.

\subsection{Sostenibilidad Basada en Complejidad}

En esta sección exponemos la experimentación realizada para validar la propuesta de métrica detallada en la sección 4.4.1, evaluando la complejidad de un conjunto de modelos de redes de decisiones. Esta experimentación la hemos realizado con dos redes de decisiones de diseño reales que reflejan el conocimiento arquitectónico, facilitadas por un grupo de investigación con el que hemos colaborado en otras líneas de investigación.

\subsubsection{Tipos de redes de decisiones}

A continuación, expondremos las diferentes topologías de redes de decisiones que hemos creado para validar nuestra propuesta de métrica. Con estos modelos representamos topologías de redes de decisiones de composición arquitectónica creciente tanto en decisiones como en relaciones de trazabilidad. En algunos de estos modelos 
hemos introducido una decisión aislada, con el fin de poder comparar la influencia de una decisión aislada en una red. Estos modelos los representamos a continuación.

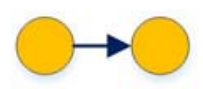

Model 1

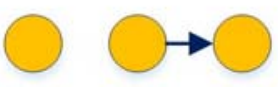

Model 2

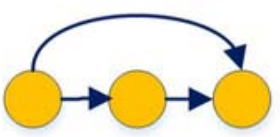

Model 3

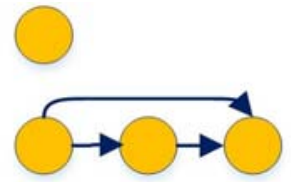

Model 4

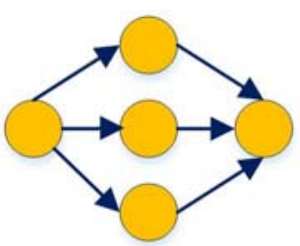

Model 5

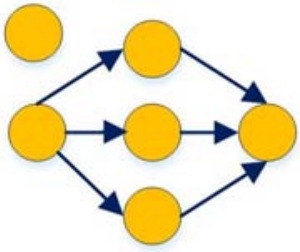

Model 6

Figura 5.1. Redes de decisiones muy pequeñas a las que se les incluye una decisión aislada.

En la figura 5.1 representamos los modelos Model 1 con dos decisiones conectadas, Model 2 con dos decisiones conectadas y una aislada, Model 3 con tres decisiones enlazadas, Model 4 con tres decisiones conectadas y una aislada, Model 5 con decisiones conectadas con múltiples caminos y finalmente Model 6 con decisiones conectadas con múltiples caminos más una aislada.

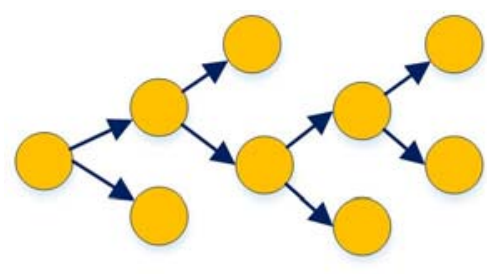

Model 7

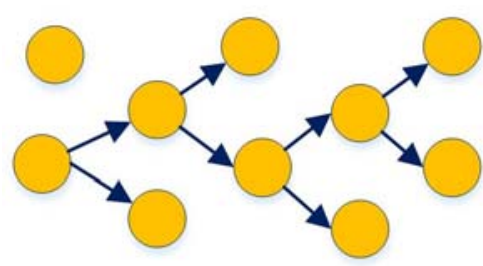

Model 8

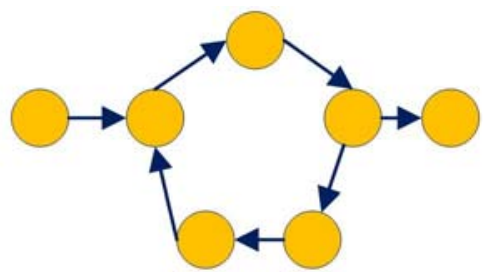

Model 9

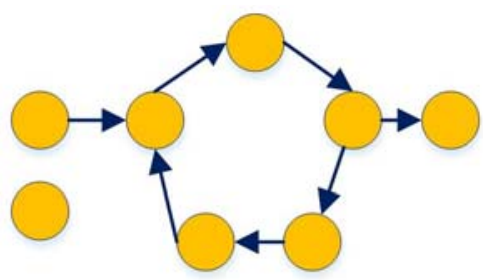

Model 10

Figura 5.2. Redes en árbol y con un solo ciclo a las que se les incluye decisiones aisladas.

También proponemos topologías en árbol y con un ciclo. En la figura 5.2 representamos los modelos Model 7, red en árbol con cinco decisiones finales. Model 8, 
red en árbol con una decisión asilada. Model 9, red con un ciclo. Model 10, red con un ciclo y una decisión aislada.

A las topologías indicadas anteriormente, incluimos otras con múltiples ciclos y caminos alternativos entre decisión inicial y final. En la figura 5.3 mostramos Model 11 con ciclos y caminos alternativos, Model 12 con ciclos, caminos alternativos y una decisión aislada, Model 13 con múltiples ciclos y múltiples caminos. Finalmente, el Model 14 se corresponde con una red con múltiples ciclos y múltiples caminos con una decisión aislada.

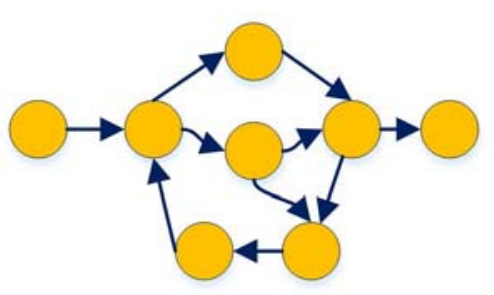

Model 11

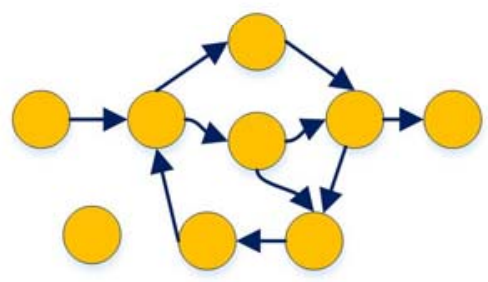

Model 12

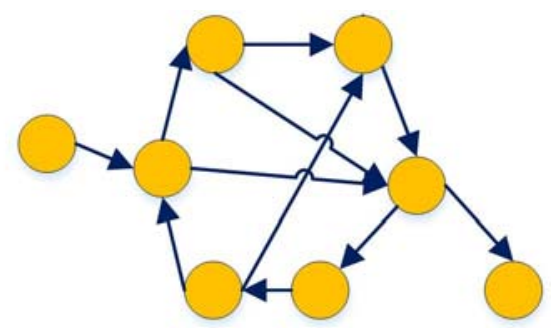

Model 13

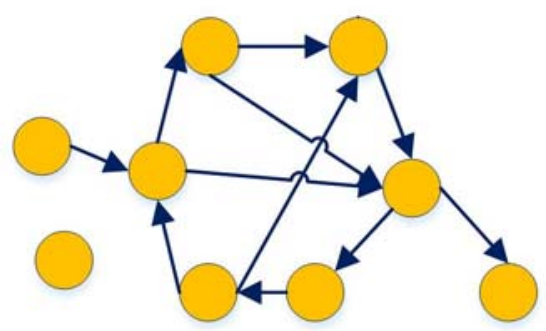

Model 14

Figura 5.3. Redes cíclicas con múltiples caminos y sin decisiones aisladas y con ellas.

En cada uno de los modelos anteriores hemos aumentando el número de decisiones no aisladas. Además, a cada modelo propuesto le hemos añadido una decisión aislada. A continuación, proponemos modelos en los que, partiendo de dos de los modelos ya propuestos, mantengamos el mismo número de decisiones y cambie el número de relaciones. El objetivo de esta propuesta es poder evaluar cómo influye una variación en el número de relaciones. Los modelos elegidos son Model 7 y Model 13, tal y como mostramos a continuación.

Además, planteamos variaciones a las topologías de árbol para evaluar como varía la complejidad en función del número de relaciones. Por ello, y tomando como origen Model 7 y mantenemos el mismo número de decisiones, incrementamos dos relaciones y variamos la topología. En la figura 5.4 mostramos Model 7 y los nuevos modelos. En 
Model 15 convertimos dos decisiones sin dependientes en decisiones con dependencia. En Model 16 pasamos a tener una decisión con dependencia más, y añadimos una relación a una decisión que pasará a tener tres decisiones dependientes. En Model 17 tenemos dos decisiones con tres dependientes.

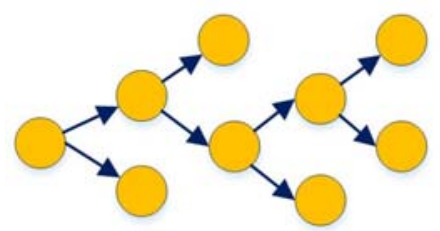

Model 7

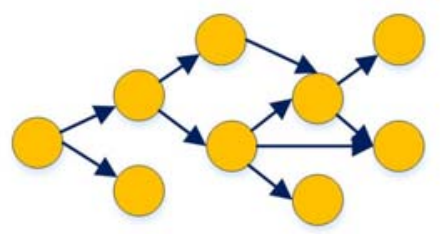

Model 16

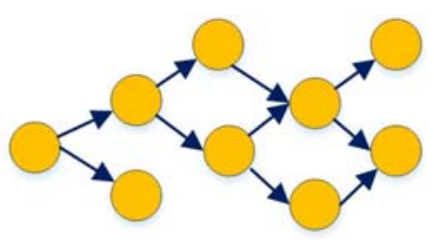

Model 15

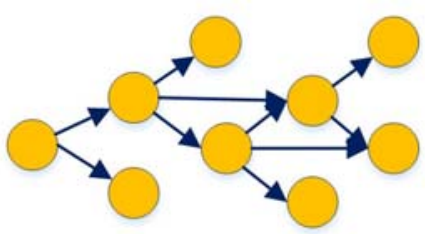

Model 17

Figura 5.4. Tres variantes de modelos basados en el modelo Model 7.

Además, para evaluar la evolución de la complejidad cuando se van eliminando ciclos, partimos de Model 13 y generamos nuevas topologías con menos ciclos. En la figura 5.5 mostramos Model 13, Model 18 con una relación menos, Model 19 tiene dos relaciones menos y, por último, Model 20 tiene tres relaciones menos que Model 13.

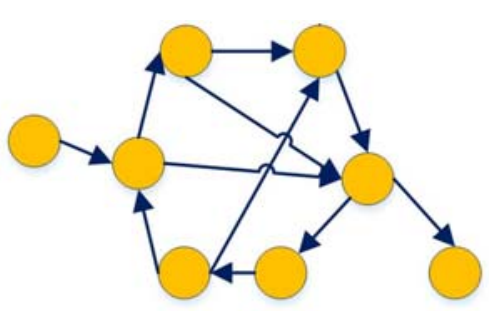

Model 13

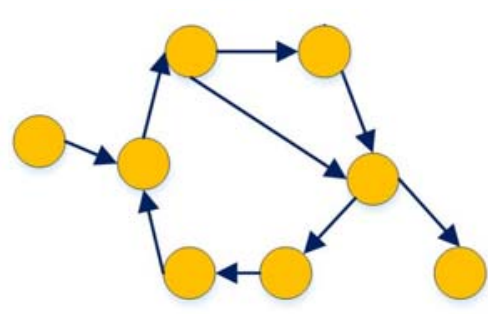

Model 19

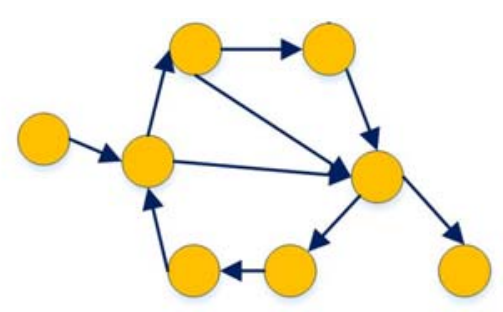

Model 18

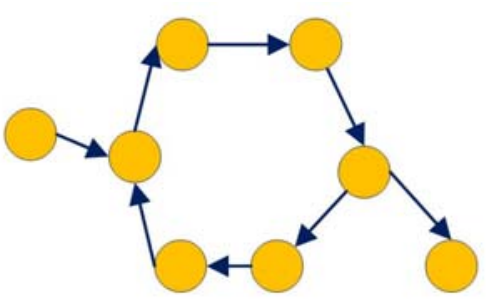

Model 20

Figura 5.5. Tres variantes de modelos basados en el modelo Model 13. 


\subsubsection{Resultados obtenidos con los modelos propuestos}

En esta sección mostraremos los valores de complejidad obtenidos por nuestra aplicación Java Architectural Design Decision Network Metrics (ADDN-Metrics), para cada una de las topologías indicadas en la sección anterior. Esta aplicación integra la métrica $A D D N-C$ y la hemos construido para validar la propuesta de métrica de la sección 4.4.1, y cuyo diseño está en el Anexo III y su codificación en el Anexo IV de este trabajo.

También incluimos las capturas de ventanas generadas por nuestra aplicación, con el objetivo de visualizar la red y analizar los valores obtenidos según su topología. En el Anexo $\mathrm{V}$ hemos detallado el formato del fichero de configuración que $A D D N$-Metrics requiere para cargar todos los elementos arquitectónicos que caracteriza cada red.

Para obtener estos datos, hemos tomado como factor de corrección configurable $\alpha$ el valor de 0,5 para todos los modelos evaluados y cuyas capturas mostramos a continuación. Hemos elegido este valor por estar en la mitad del rango [0,1], porque consideramos que la gestión de una decisión aislada se corresponde con la mitad de complejidad que requiere una decisión no aislada. Por cada modelo de topología analizado, hemos capturado su representación gráfica y en la sección 5.1.5 motivaremos los resultados obtenidos.

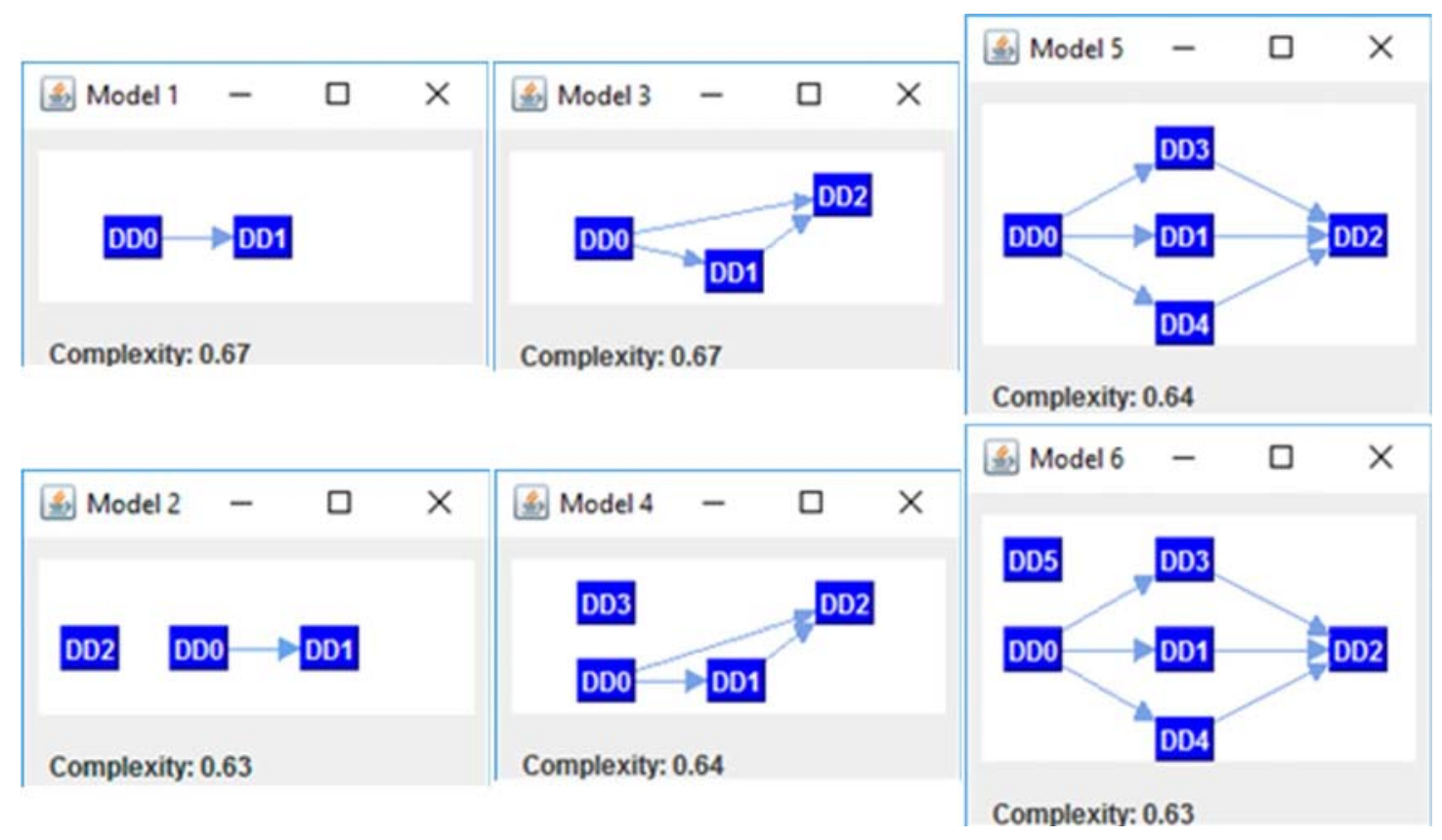

Figura 5.6. Estimación de la medida de complejidad para redes muy pequeñas. 
En la figura 5.6 mostramos la estimación de medida de complejidad para cada uno de los modelos Model 1, Model 2, Model 3, Model 4, Model 5 y Model 6. A continuación, realizamos el mismo proceso de estimación para los modelos Model 7, Model 8, Model 9 y Model 10, como vemos en la figura 5.7.

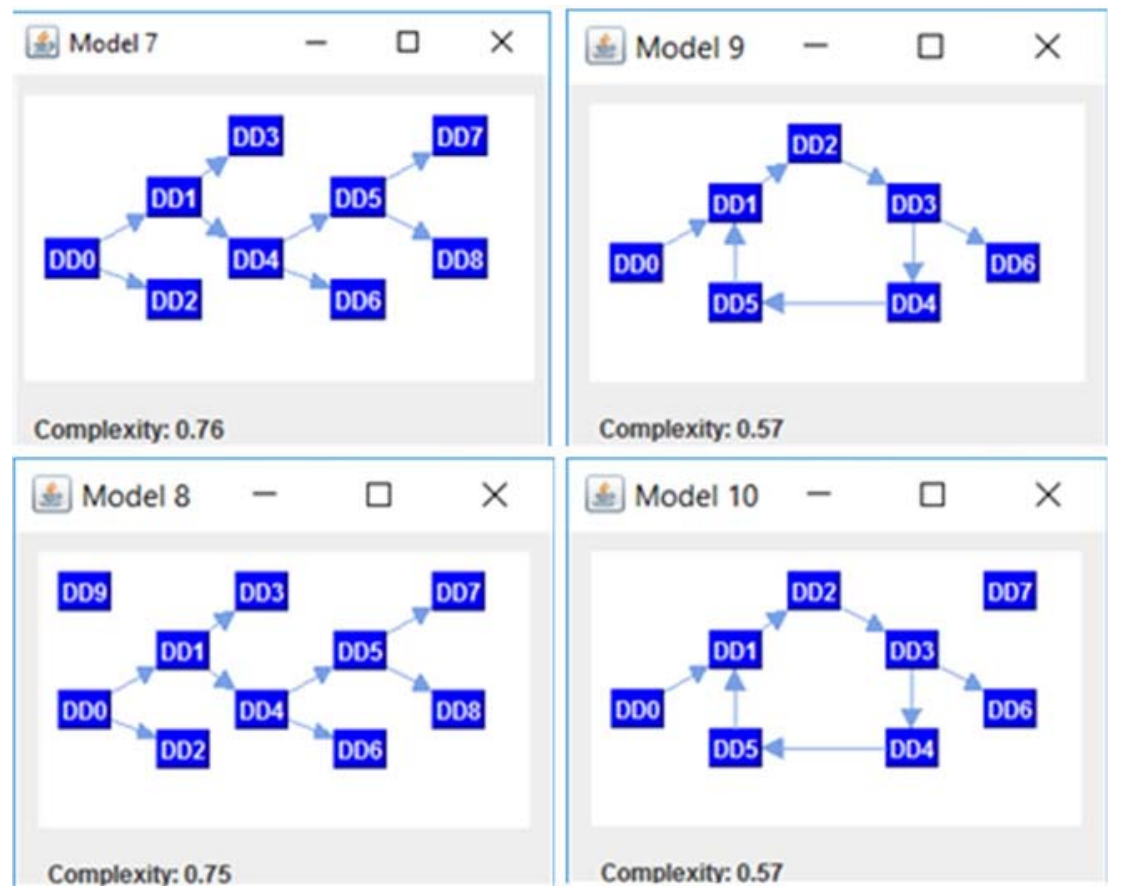

Figura 5.7. Estimación de la medida de complejidad para redes con árboles y ciclos.

En la figura 5.8 indicamos la estimación de medida de complejidad para los modelos propuestos Model 11, Model 12, Model 13 y Model 14.

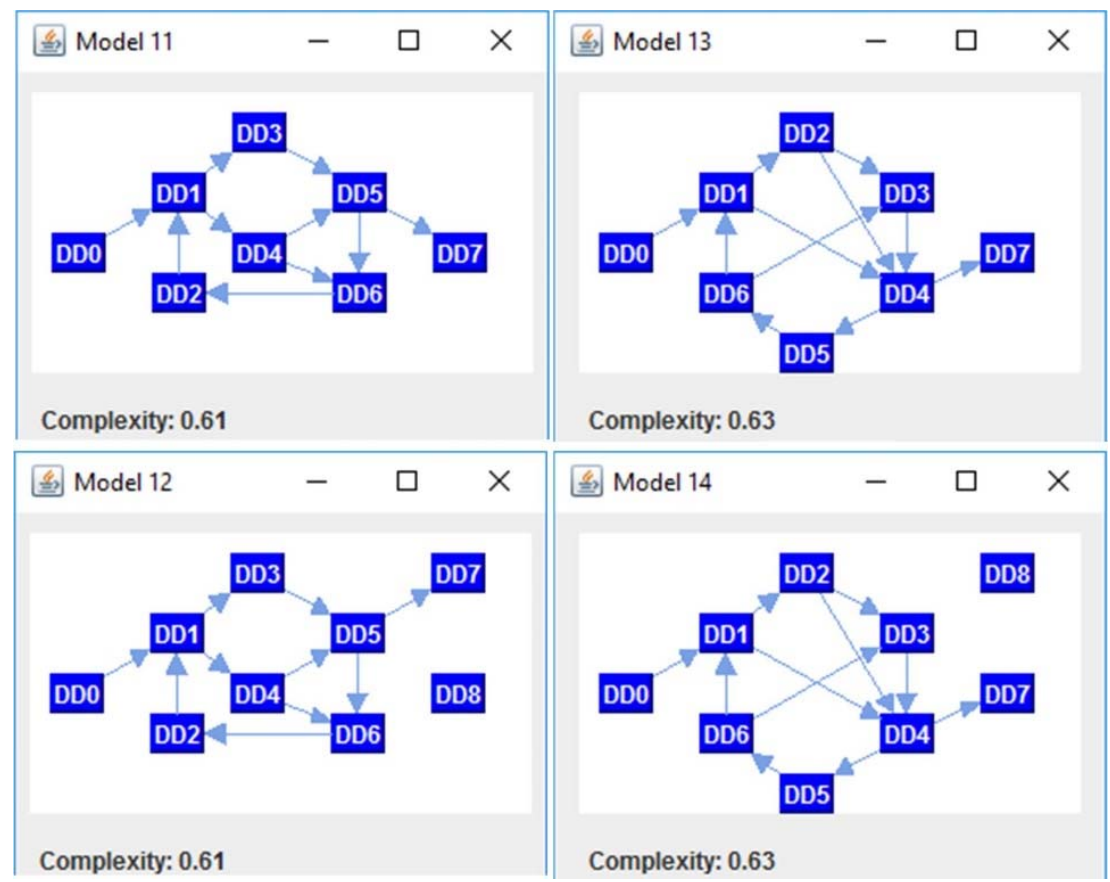

Figura 5.8. Estimación de la medida de complejidad para redes que tienen múltiples ciclos. 
La figura 5.9 refleja las capturas de las ventanas Java de las variaciones de modelos propuestas, junto a la medida de complejidad de ADDN-C para las diferentes variantes de red de tipo árbol.

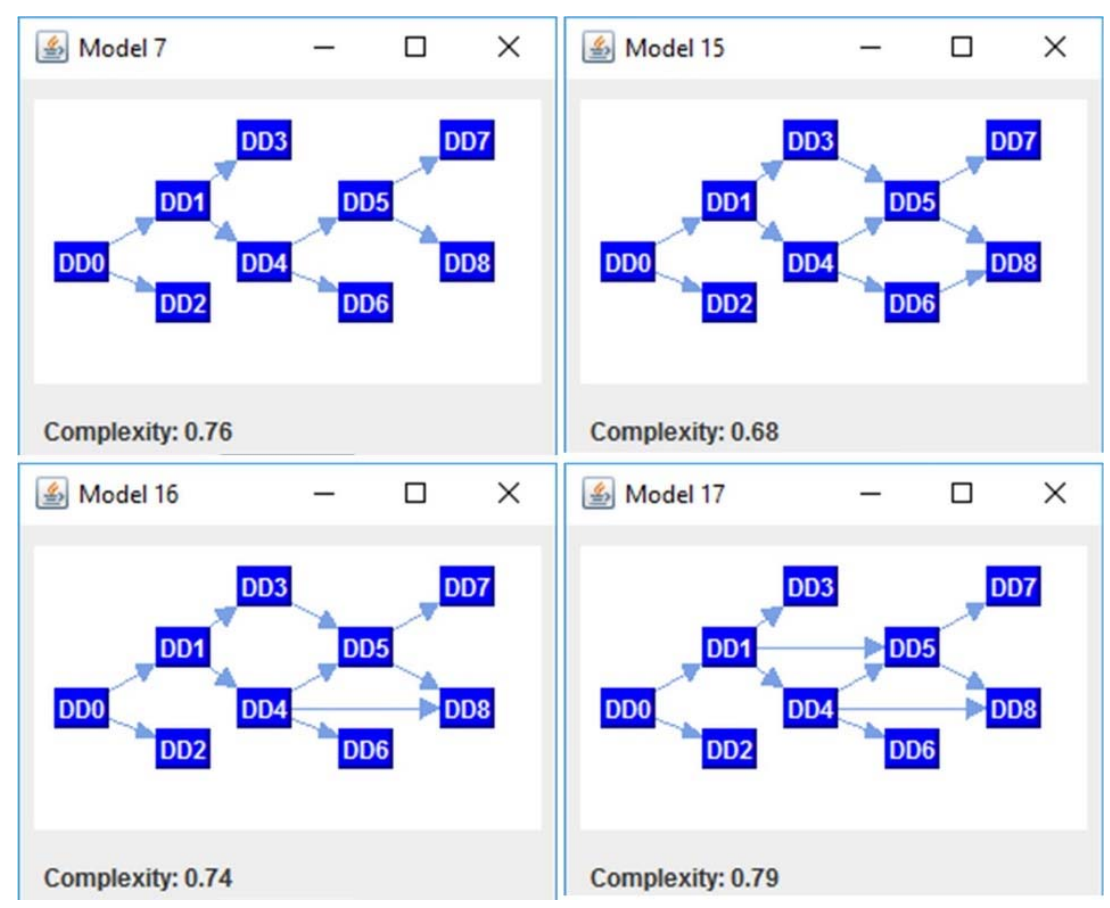

Figura 5.9. Estimación de la complejidad para las variantes de tipo árbol.

En la figura 5.10 representamos la estimación de la complejidad para variantes del modelo Model 13.

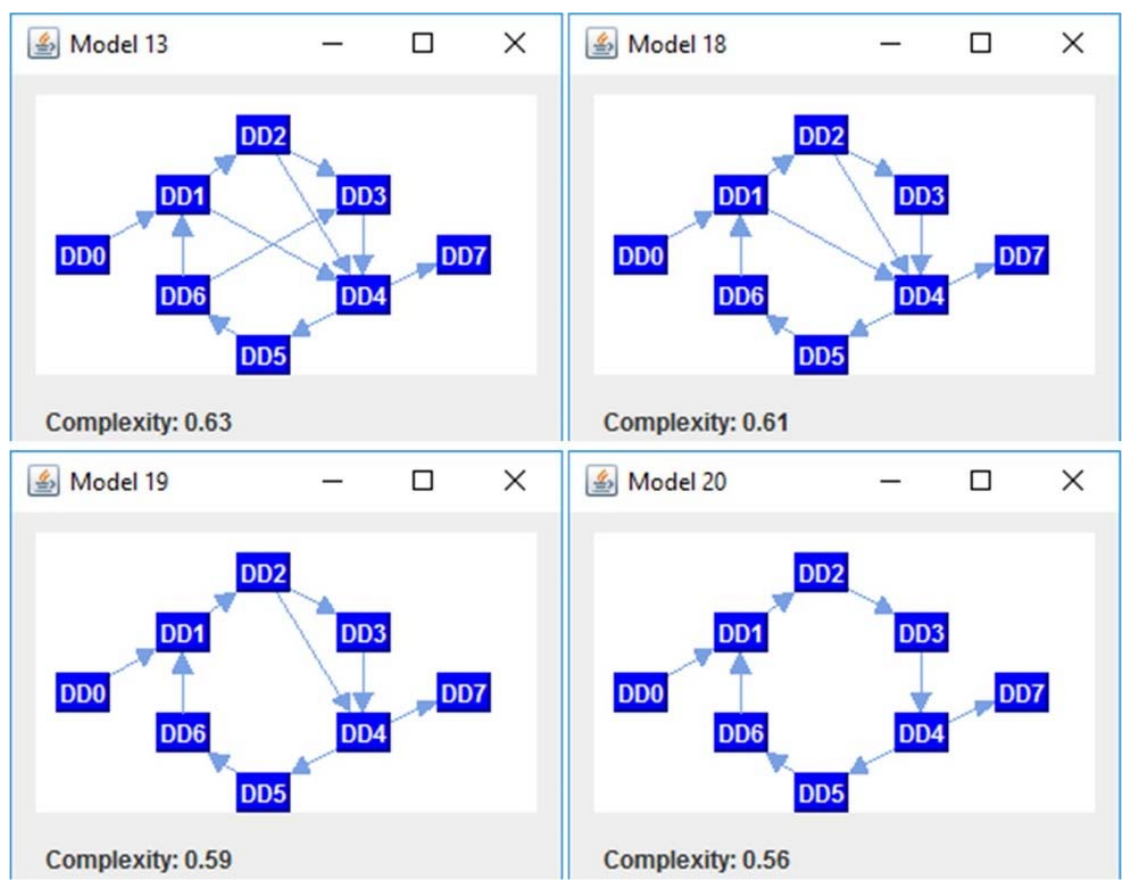

Figura 5.10. Estimación de la complejidad para las variantes de redes cíclicas. 
A modo de resumen, en la tabla 5.1 indicamos los valores de complejidad obtenidos por $A D D N$-Metrics para los modelos de redes propuestos sin decisiones aisladas, en la que hemos ordenado las topologías en función del número de decisiones y de relaciones.

Tabla 5.1. Valores de complejidad obtenidas en redes sin decisiones aisladas.

\begin{tabular}{l|l} 
Modelo & Complejidad
\end{tabular}

Redes con topologías ciclicas

\begin{tabular}{|l|c|}
\hline Model 20. Red con un ciclo. Variante de Model 13. & 0,56 \\
\hline $\begin{array}{l}\text { Model 19. Red con dos ciclos, caminos alternativos. } \\
\text { Variante de Model 13. }\end{array}$ & 0,59 \\
\hline Model 11. Red con ciclos y caminos alternativos. & 0,61 \\
\hline $\begin{array}{l}\text { Model 18. Red con tres ciclos, caminos alternativos. } \\
\text { Variante de Model 13. }\end{array}$ & 0,61 \\
\hline Model 13. Red con cuatro ciclos, caminos alternativos. & 0,63 \\
\hline
\end{tabular}

Redes con tolopogías en árbol

Model 7. Red en árbol, con cinco decisiones finales.

0,76

Model 15. Red en árbol con tres decisiones finales. Variante $\quad 0,68$ de Model 7.

Model 16. Red en árbol. Con cuatro decisiones finales y una $\quad 0,74$ decisión con tres dependencias. Variante de Model 7.

Model 17. Red en árbol. Dos decisiones con tres 0,79 dependencias. Variante de Model 7.

Con el fin de realizar un estudio comparativo de la complejidad en redes con decisiones aisladas, hemos repetido el experimento con los valores de $0,13,0,25,0,5 \mathrm{y}$ 0,75 del factor de corrección $\alpha$. Los resultados que hemos obtenido por ADDN-Metrics los exponemos en la tabla 5.2. 
Tabla 5.2. Valores de complejidad obtenidas en redes con decisiones aisladas.

\begin{tabular}{|c|c|c|c|c|}
\hline \multirow{2}{*}{ Modelo } & \multicolumn{4}{|c|}{ Complejidad } \\
\hline & $\alpha=0,13$ & $\alpha=0,25$ & $\alpha=0,5$ & $\alpha=0,75$ \\
\hline $\begin{array}{l}\text { Model } 6 . \text { Red con decisiones conectadas con } \\
\text { múltiples caminos más una aislada. }\end{array}$ & 0,59 & 0,6 & 0,63 & 0,65 \\
\hline Model 8. Red en árbol con una decisión aislada. & 0,73 & 0,74 & 0,75 & 0,76 \\
\hline $\begin{array}{l}\text { Model 10. Red con un ciclo y una decisión } \\
\text { aislada. }\end{array}$ & 0,54 & 0,55 & 0,57 & 0,58 \\
\hline $\begin{array}{l}\text { Model } 12 . \text { Red con ciclos, caminos alternativos } \\
\text { y una decisión aislada. }\end{array}$ & 0,59 & 0,59 & 0,61 & 0,62 \\
\hline $\begin{array}{l}\text { Model 14. Red con múltiples ciclos y múltiples } \\
\text { caminos con una decisión aislada. }\end{array}$ & 0,61 & 0,61 & 0,63 & 0,64 \\
\hline
\end{tabular}

\subsubsection{Experimentación con redes de tipo plataformas basadas en servicios}

Con el objetivo de cuantificar la complejidad de redes de decisiones reales, hemos estimado la complejidad de dos redes de decisiones de diseño en entornos de plataformas basadas en servicios "Service-based platform" [LYTR 12A] [LYTR 12B]. Los elementos arquitectónicos facilitados están en un formato no adaptado para $A D D N$ Metrics. Por ello ha sido necesario realizar un proceso de adaptación para que dichos datos puedan ser interpretados. Este proceso lo detallamos en el Anexo V.

Estas redes las identificamos como red SBP1 con 101 decisiones (61 conectadas y 41 aisladas) y 70 relaciones y red SBP2 con 75 decisiones (33 conectadas y 42 aisladas) y 50 relaciones y su representación gráfica completa se corresponde con los modelos representados en las figuras 5.12 y 5.16 respectivamente. Para permitir una mayor experimentación de nuestra propuesta de métrica, hemos generado dos versiones de estas redes eliminando las decisiones aisladas.

A continuación, en la figura 5.11 mostramos una versión sin decisiones aisladas de la red $S B P 1$ y la identificamos como $S B P 3$. 
Capítulo 5. Experimentación

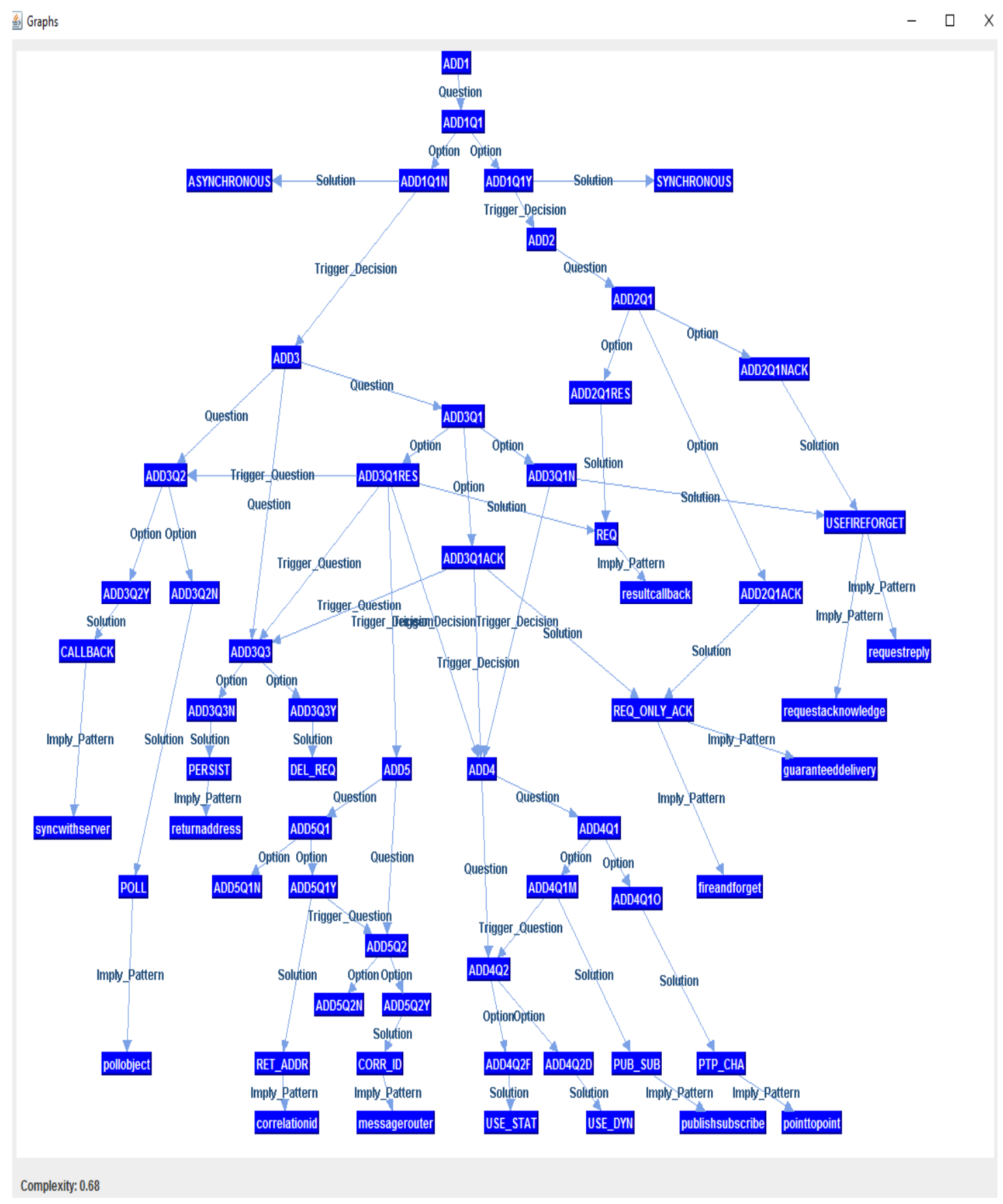

Figura 5.11. Red SBP3 visualizada por $A D D N-M e t r i c s$.

La red SBP1 la visualizamos en la figura 5.12. 


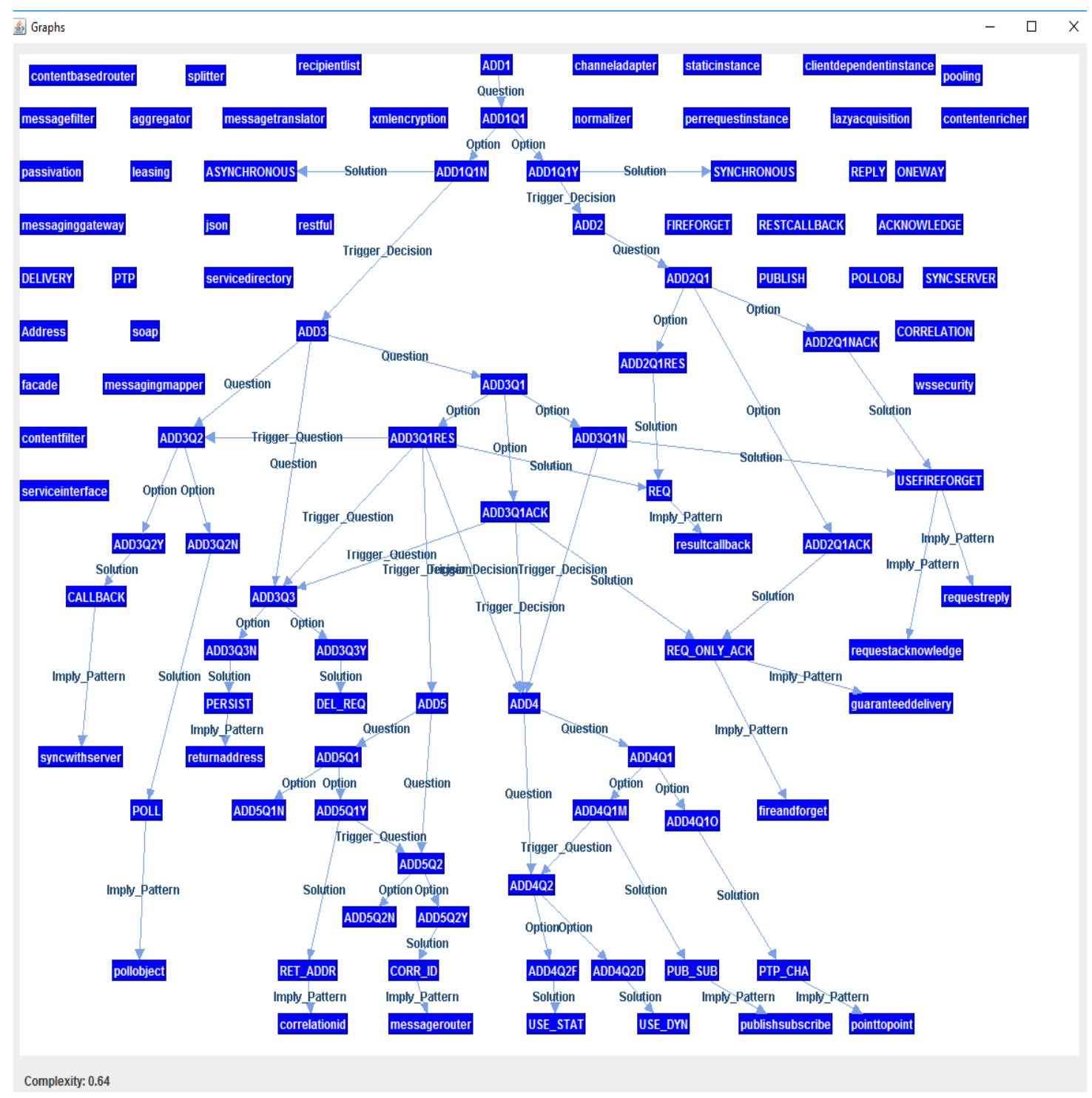

Figura 5.12. Red SBP1 visualizada por $A D D N-$ Metrics

En este tipo de redes, donde el número de decisiones es muy elevado (101), es necesario que el arquitecto software realice un esfuerzo de diseño gráfico muy alto para determinar la posición $(\mathrm{X}, \mathrm{Y})$ donde cada nodo debe posicionarse. A pesar que la aplicación permite seleccionar y mover cada nodo una vez generado el grafo, hemos considerado necesario utilizar una herramienta que represente grafos de manera automática. Por ello, hemos optado por utilizar la aplicación Neo4j, que es una base de datos orientada a grafos, y que distribuye automáticamente cada nodo en el espacio. En Neo4j también es posible mover cada nodo de manera manual, una vez creado el grafo. La representación gráfica de las redes que hemos mostrado en las figuras 5.11 (red SBP3) y 5.12 (red SBP1) en la aplicación Neo4j se corresponde con las figuras 5.13 (red SBP3) y 5.14 (red SBP1) respectivamente. 


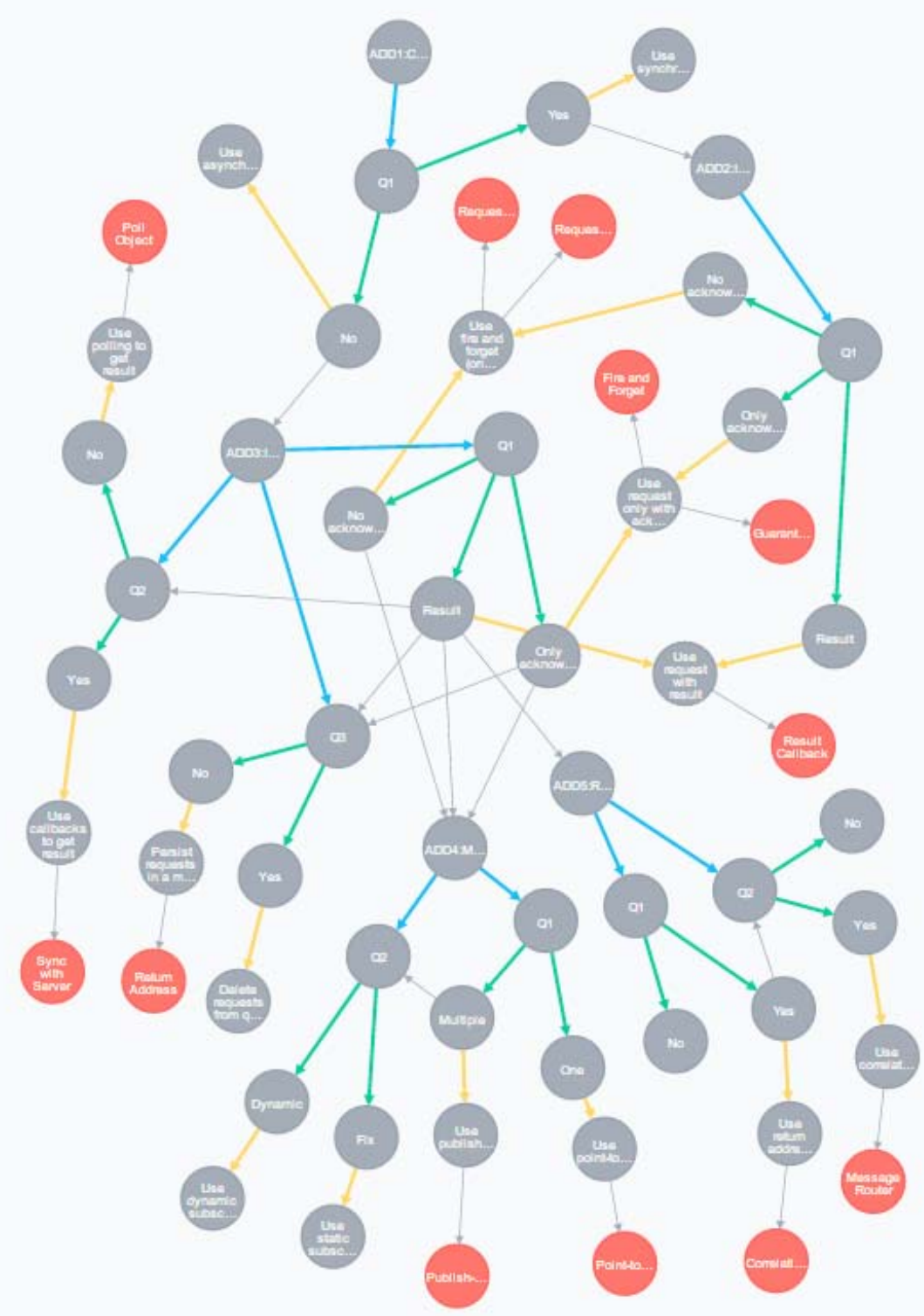

Figura 5.13. Red SBP3 visualizada por Neo4J.

Como observamos en las figuras 5.13 y 5.14, Neo4J permite configurar la representación para diferenciar visualmente mediante colores los diferentes tipos de nodos y diferentes tipos de relación, según los descriptores que muestra en la parte superior de ambas figuras. La asignación de colores y grosores de líneas la realizamos mediante la configuración del fichero "graphstyle.grass", y que está detallado en la Anexo V de este trabajo. 


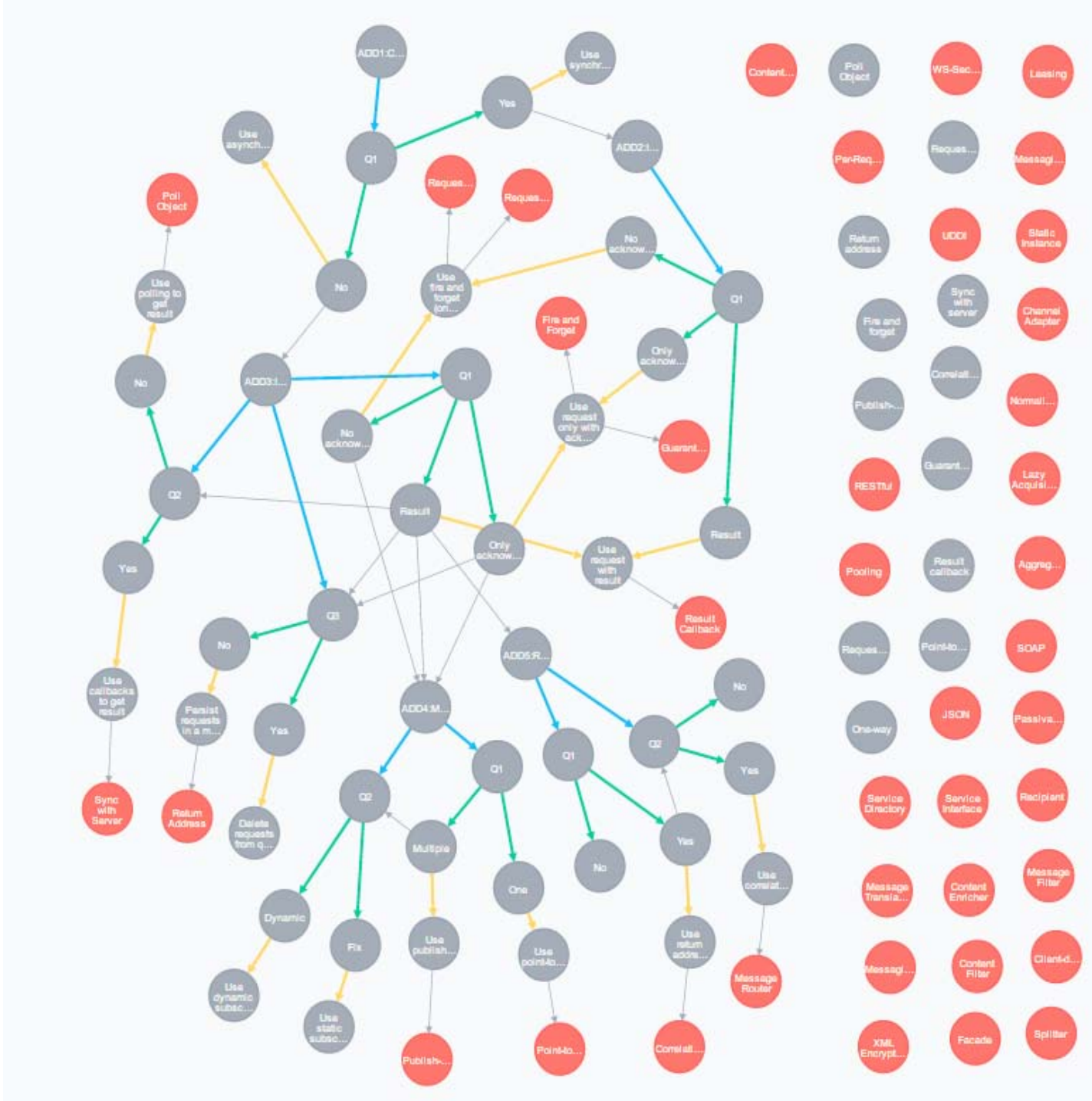

Displaying 101 nodes, 70 relationships (completed with 70 additional relationships).

Figura 5.14. Red SBP1 visualizada por Neo4J.

Otra red de decisiones de diseño que estudiamos es la red SBP2 y visualizada en la figura 5.16. En este caso, hemos generado una red sin decisiones aisladas con el fin de poder experimentar con otro modelo de red como visualizamos en la figura $5.15 \mathrm{y}$ a la que identificamos como SBP4. En ambos casos, ha sido necesario realizar sendos procesos de adaptación de los datos arquitectónicos facilitados y que está detallado en el Anexo V de este trabajo. 


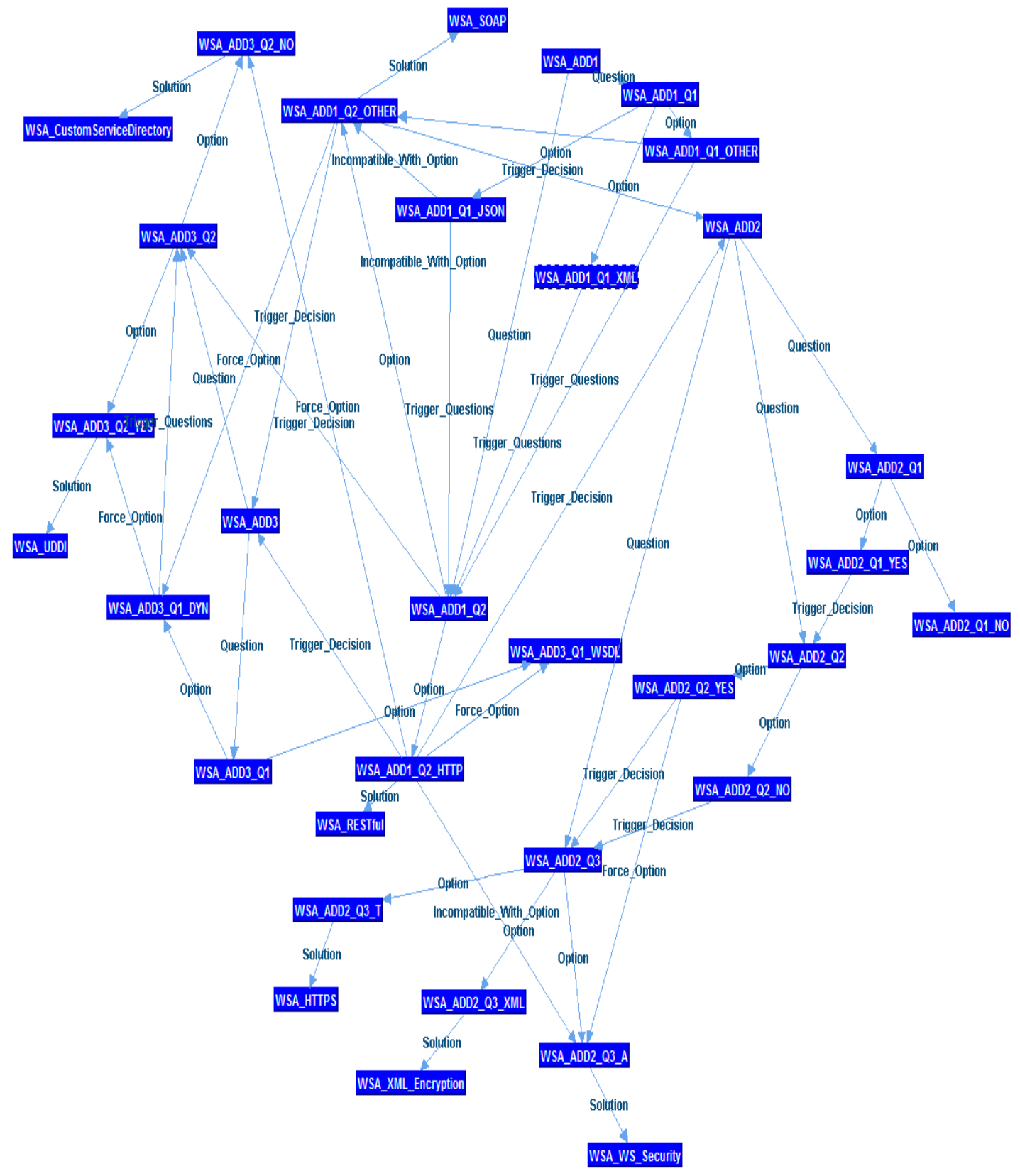

Figura 5.15. Red SBP4 visualizada por ADDN-Metrics.

A continuación, mostramos la red SBP2 con todos sus elementos arquitectónicos en la figura 5.16. 


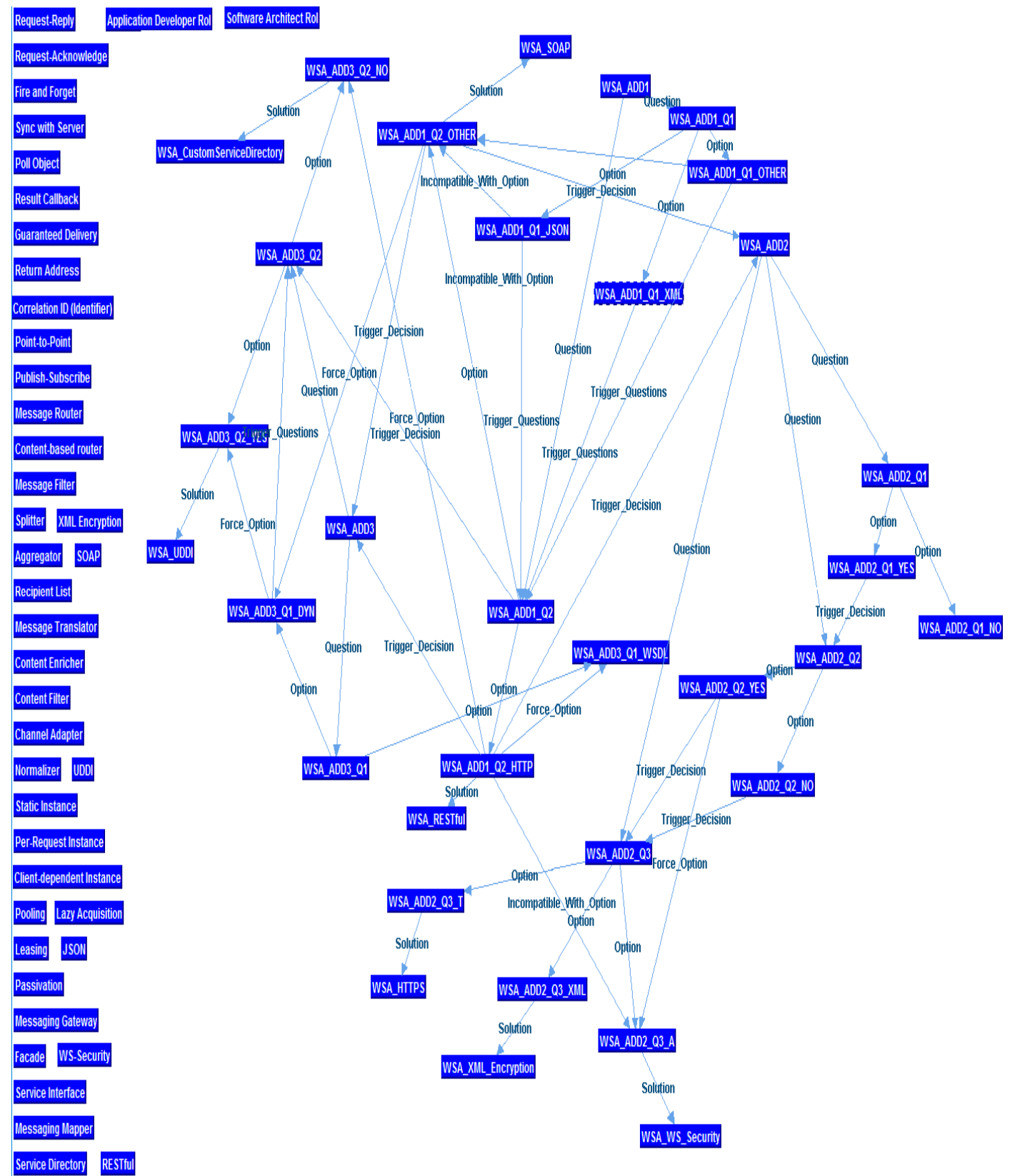

Figura 5.16. Red $S B P 2$ visualizada por $A D D N$-Metrics.

Como ya hemos comentado anteriormente, el diseño gráfico de esta red requiere un gran esfuerzo al arquitecto software. Por ello, también hemos representado esta última red mediante la aplicación Neo4j. A continuación, mostramos estas redes de tal manera que las figuras 5.15 y 5.16 se corresponden con las figuras 5.17 y 5.18 respectivamente. 


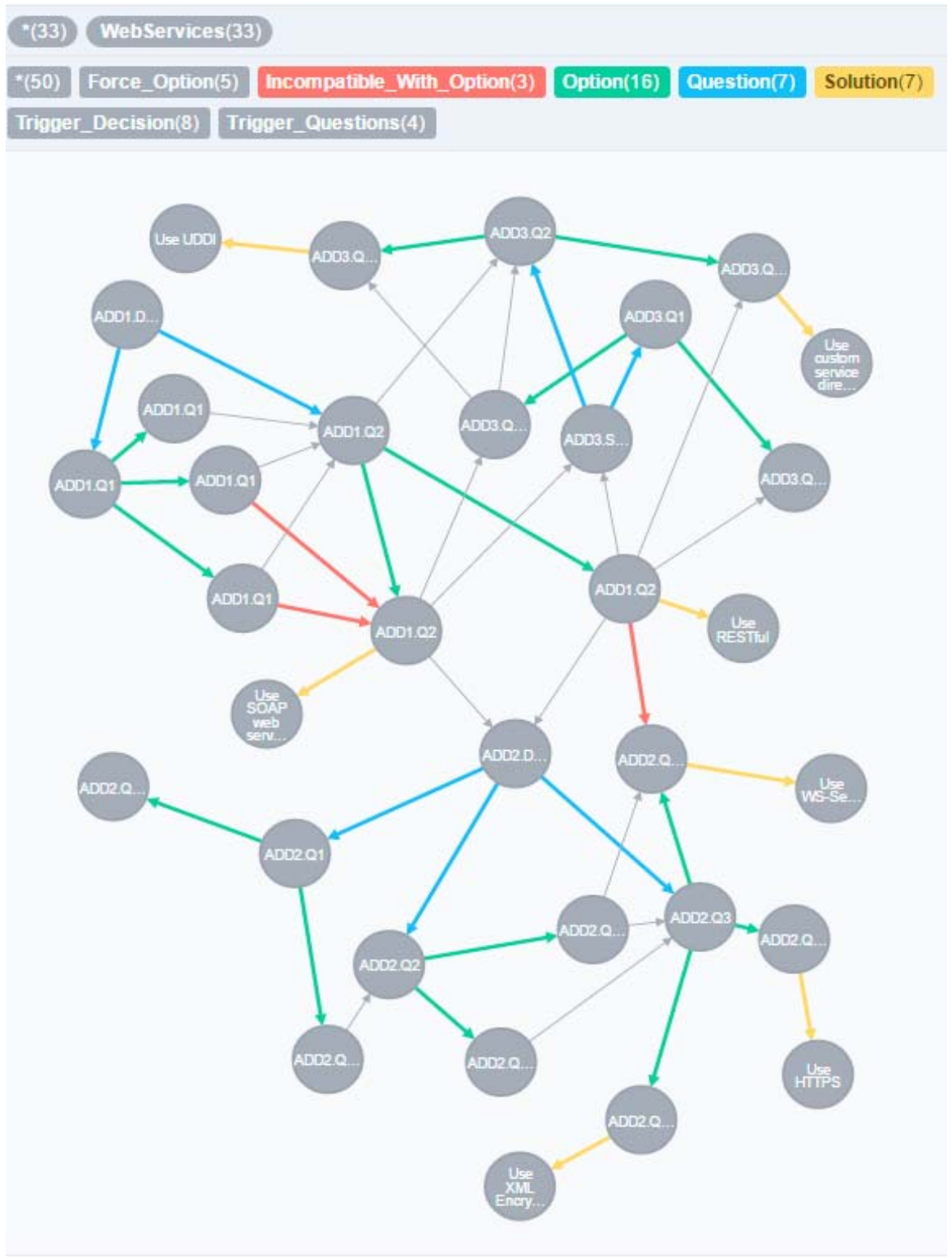

Displaying 33 nodes, 50 relationships (completed with 50 additional relationships).

Figura 5.17. Red SBP4 visualizada por Neo4J.

Se observa varias leyendas en las cabeceras de las figuras 5.17 y 5.18 que describen el contenido de esta figura. En ella vemos cómo se agrupan los nodos y cuantos hay (primera fila) y cuantas relaciones y como se agrupan (segunda línea). Dado que SBP4 se corresponde con un modelo QOC, las relaciones tienen etiquetas que nos permiten agruparlas. Además, Neo4j nos permite asignar colores a los elementos arquitectónicos para facilitar su visualización. En las leyendas de ambas figuras se indica que color hemos asignado a cada grupo de nodos y relaciones. La asignación de colores, incluso el grosor de las líneas que representan las relaciones, se realiza mediante la configuración del fichero "graphstyle.grass". En el Anexo V de este trabajo mostramos el contenido de este fichero, con un código similar al formato de estilos CSS en páginas web HTML. 


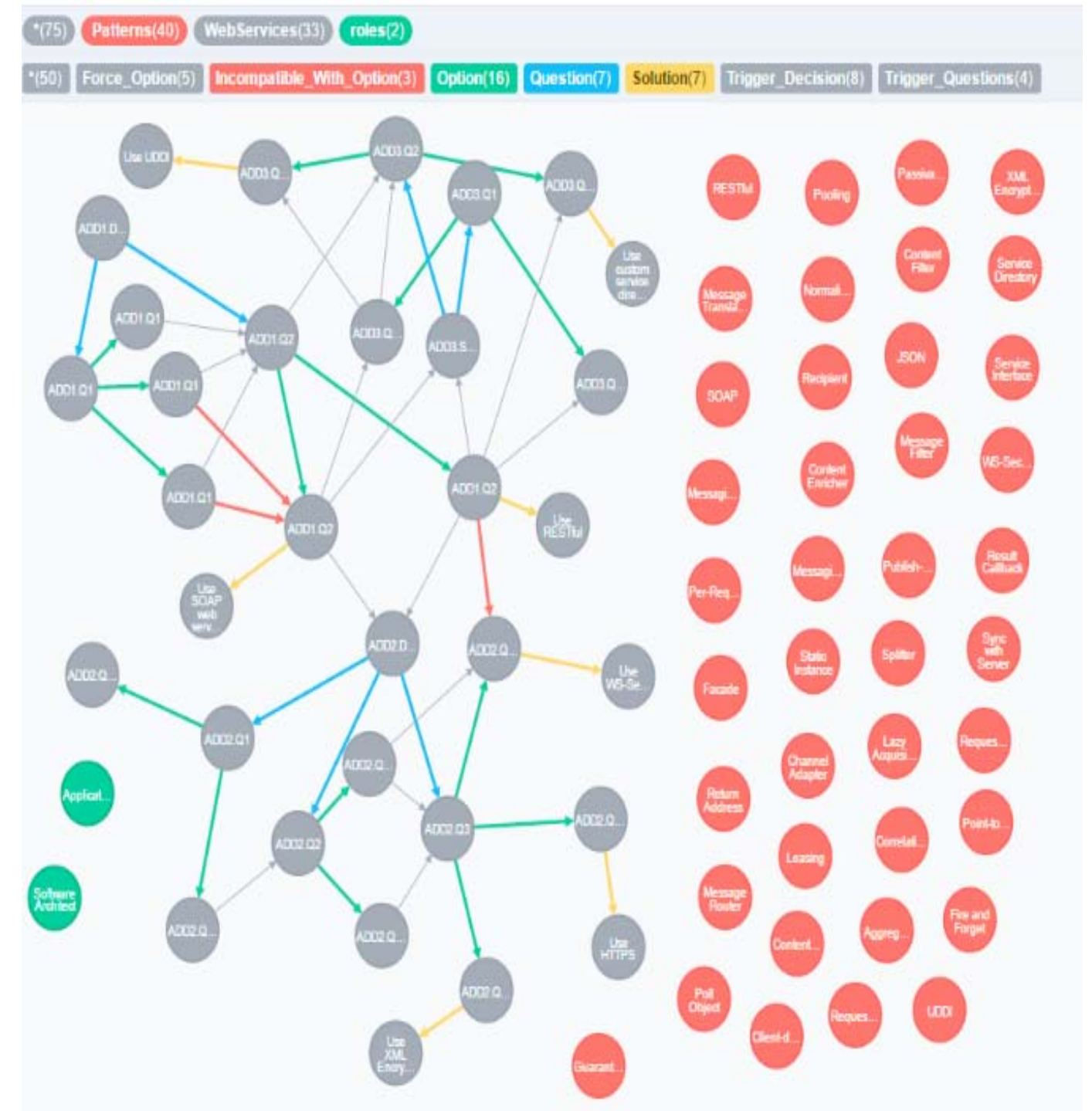

Displaying 75 nodes, 50 relationships (completed with 50 additional relationships).

Figura 5.18. Red SBP2 visualizada por Neo4J

Por último, para permitir una mayor experimentación con este tipo de redes, hemos unido $S B P 1$ y $S B P 2$ completas y el resultado lo mostramos en la figura 5.19. La red resultante SBP5 está formada por 136 decisiones y con 120 relaciones. 

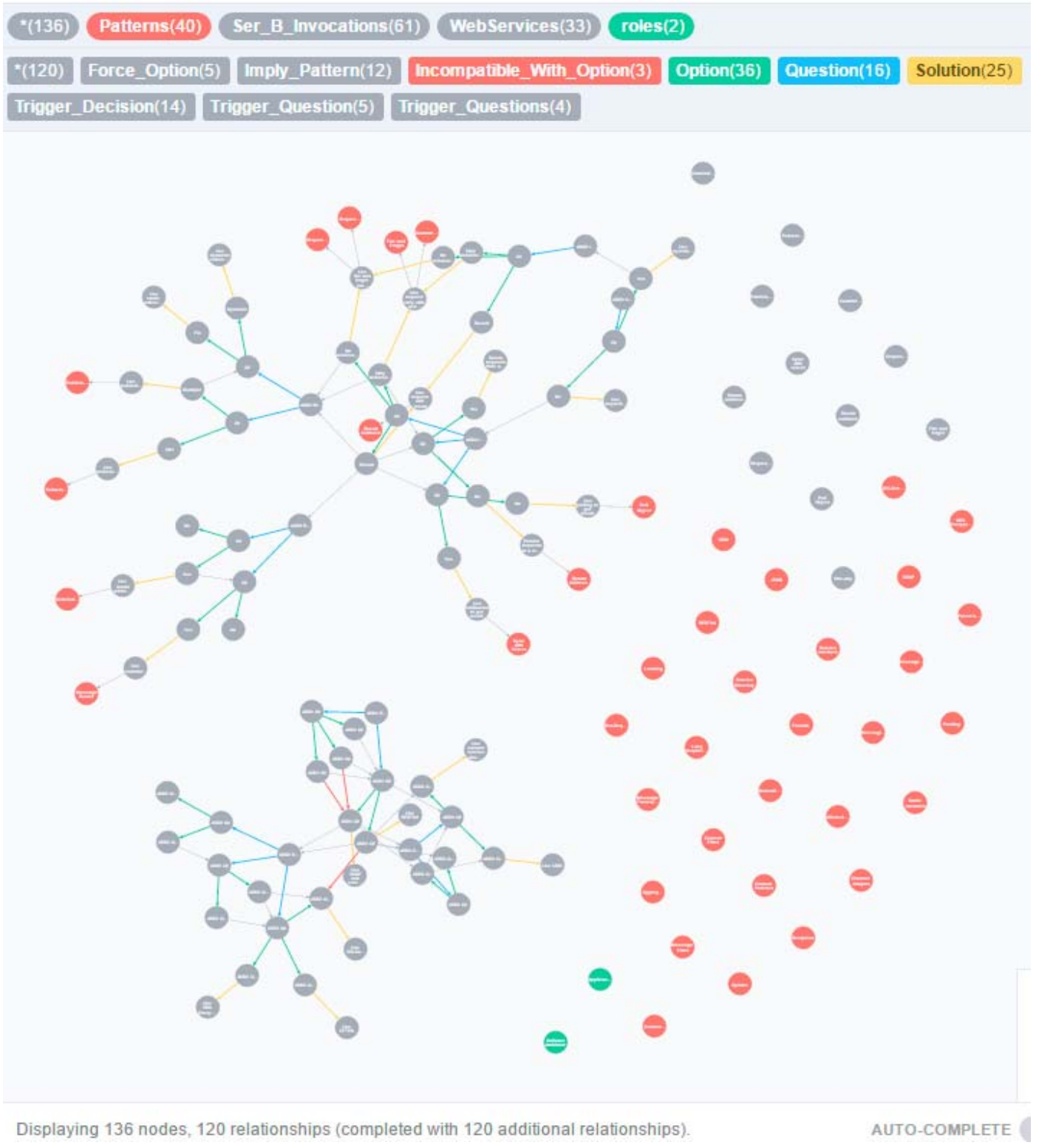

Figura 5.19. Red SBP5 visualizada por Neo4J.

\subsubsection{Resultados con redes de decisiones complejas}

Al estimar la complejidad de estas redes con nuestra métrica ADDN-C, obtenemos los valores de complejidad que detallamos en la tabla 5.3.

Tabla 5.3. Valores de complejidad obtenidas en redes SBP sin decisiones aisladas.

Red SBP3, con 50 decisiones conectadas y 70 relaciones. 
Con el fin de realizar un estudio comparativo de la complejidad en redes de decisiones con decisiones aisladas, hemos repetido el experimento con diferentes valores del factor de corrección $\alpha$. Además del valor de 0,5 , también hemos considerado los valores del factor $\alpha 0,13,0,25$ y 0,75 . Los resultados obtenidos por nuestra métrica ADDN-C con estos tres valores los reflejamos en la tabla 5.4

Tabla 5.4. Valores de complejidad obtenidas en redes $S B P$ con decisiones aisladas.

\begin{tabular}{|l|c|c|c|c|}
\hline Modelo & \multicolumn{4}{|c|}{ Complejidad } \\
\hline $\begin{array}{l}\text { Red SBP1, con 40 decisiones aisladas y 61 } \\
\text { decisiones conectadas mediante 70 relaciones. }\end{array}$ & 0,55 & 0,58 & 0,64 & 0,7 \\
\hline $\begin{array}{l}\text { Red SBP2, con 42 decisiones aisladas y 33 } \\
\text { decisiones conectadas mediante 50 relaciones. }\end{array}$ & 0,52 & 0,56 & 0,64 & 0,72 \\
\hline $\begin{array}{l}\text { Red SBP5, con 136 decisiones. 42 son } \\
\text { decisiones aisladas. }\end{array}$ & 0,6 & 0,62 & 0,66 & 0,7 \\
\hline
\end{tabular}

\subsubsection{Interpretación de los resultados}

A continuación, interpretaremos los resultados obtenidos en las secciones 5.1.2 y 5.1.4 de este trabajo en varios niveles. En primer lugar, será una interpretación general a todas las redes analizadas. Posteriormente expondremos nuestra interpretación para redes que no tienen decisiones aisladas, y finalmente procedemos a hacerlo para redes con decisiones aisladas.

Interpretación general: La métrica ADDN-C propuesta evalúa la complejidad de la estructura de una red de decisiones. Los mayores valores de complejidad representan decisiones con un mayor número de hijos.

De los resultados obtenidos, se deduce que el mantenimiento en redes con decisiones aisladas resulta más fácil de mantener porque su complejidad es menor. En aquellas redes donde la inclusión o eliminación de una relación no modifica el número de decisiones que tengan otras dependientes, la complejidad no varía. Sin embargo, si se incrementa el número de enlaces salientes de una decisión, la complejidad puede aumentar independientemente del número de ciclo. 
Resultados de redes sin decisiones aisladas: De los datos obtenidos en la tabla 5.1, generamos su representación gráfica de la figura 5.20, y obtenemos las siguientes interpretaciones, donde destacamos en color verde los valores relacionados con redes con ciclos y en color rojo los valores correspondientes a topologías en árbol.

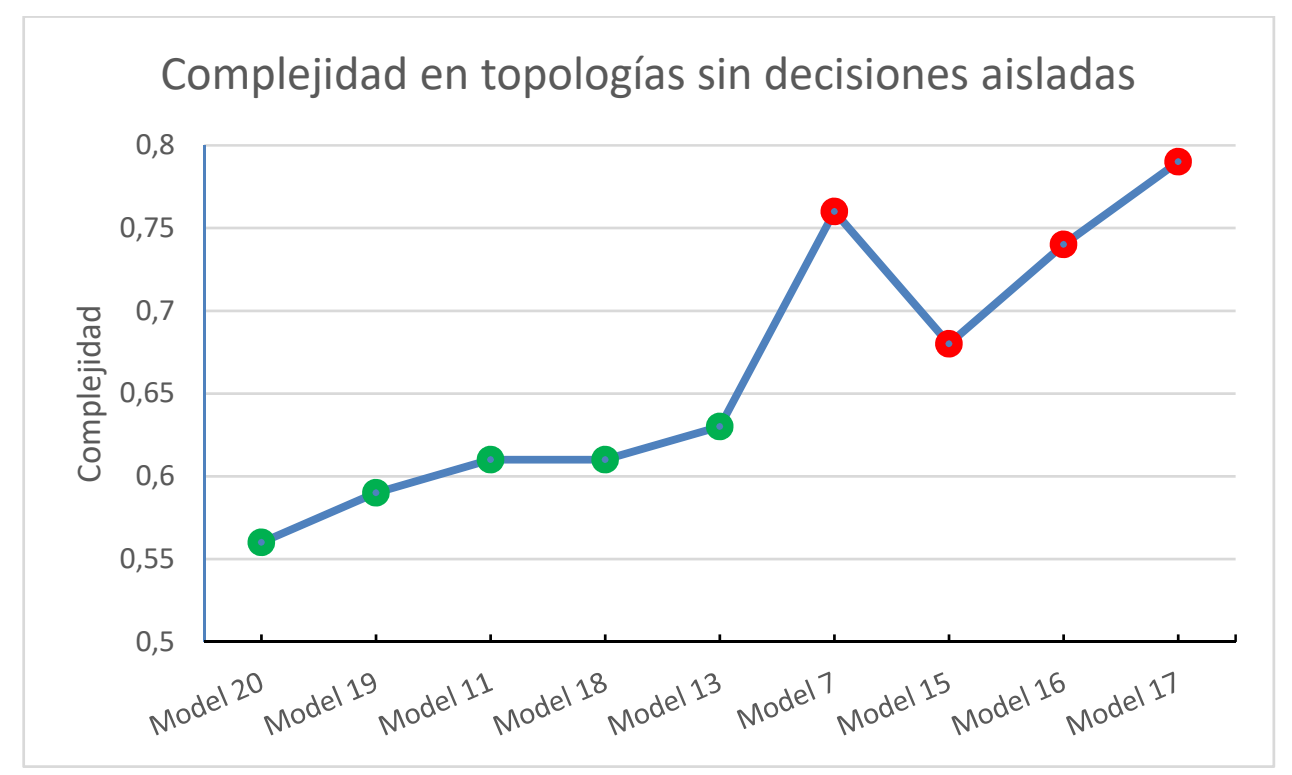

Figura 5.20. Gráfica de complejidad de diferentes topologías sin decisiones aisladas

En la figura 5.20 observamos que en las topologías con ciclos (color verde), la complejidad aumenta según aumentamos el número de decisiones y trazas. Por último, en las topologías en árbol (color rojo), la complejidad aumenta también a medida que aumenta el número de elementos arquitectónicos, con la excepción de la topología Model 7. A continuación procedemos a realizar un análisis más profundo de las redes en árbol y cíclicas.

Las topologías de tipo árbol (Model 7, Model 15, Model 16 y Model 17) tienen una complejidad mayor (figura 5.20) que el resto de topologías. Esto es debido a la existencia de múltiples dependencias. Las topologías 7 y 17 tienen una mayor complejidad que la 15 y 16 debido a que se le incrementa el número de decisiones finales y el número de decisiones con más hijos, manteniendo estable el número de nodos de la red. La evolución de la complejidad de estos cuatro modelos se muestra en la figura 5.21. 


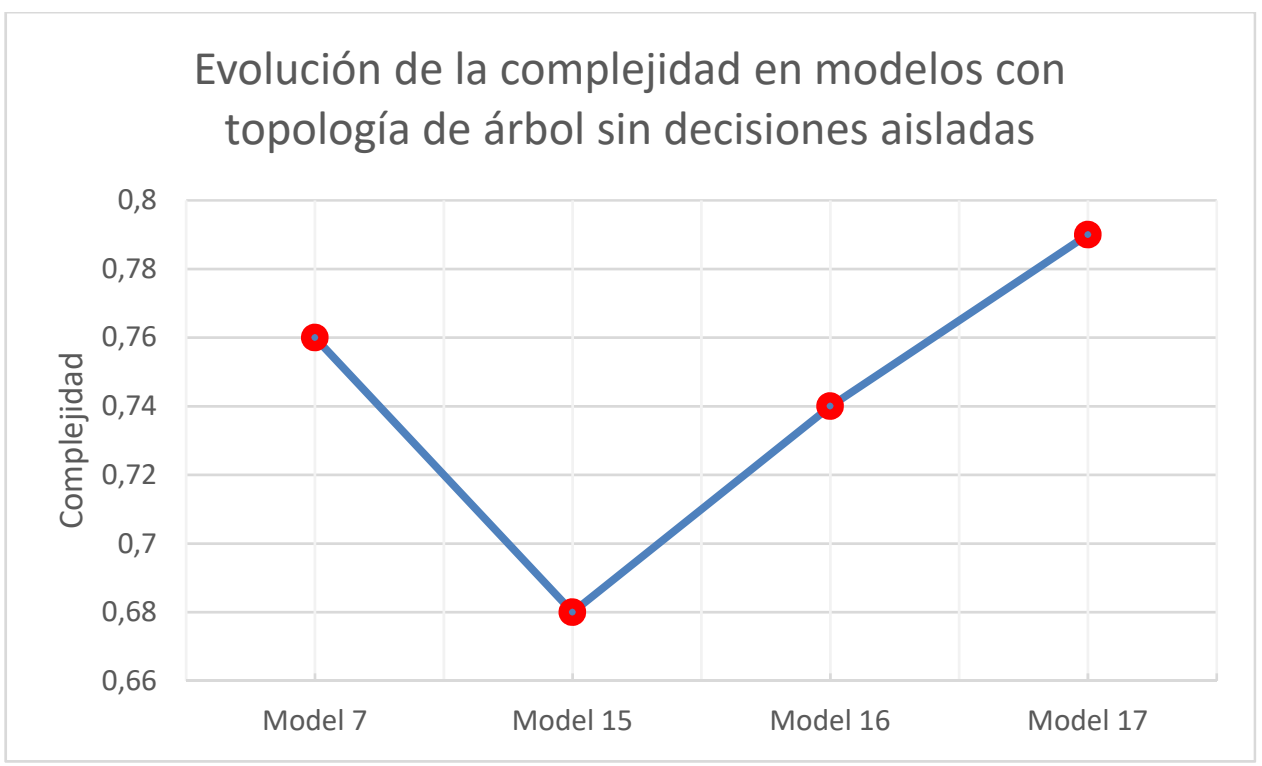

Figura 5.21. Comparativa de complejidad en variantes de redes con topología de árbol.

La introducción de dos relaciones en Model 15 respecto a Model 7 hace que la complejidad calculada sea menor. En Model 7 existen 5 decisiones que no tienen dependencias y 4 decisiones que tienen dos dependientes cada una de ellas. Con la incorporación de dos relaciones en Model 15, no aumentamos el número de decisiones con dos o más dependencias, pero si el número de elementos arquitectónicos. Por ello, la complejidad calculada es menor. Cuando las dos nuevas relaciones provocan la aparición de al menos una decisión con tres decisiones dependientes, como ocurre en Model 16 y Model 17, la complejidad es mucho mayor frente a Model 15, a pesar que estos tres modelos tienen exactamente el mismo número de elementos arquitectónicos. La justificación de este aumento está en que se ha aumentado la complejidad que introduce cada decisión con tres dependencias, en relación al número de elementos arquitectónicos del modelo. La complejidad de Model 17 es mayor que la de Model 16 dado que el número de decisiones con tres dependientes es mayor.

En cuanto a las topologías cíclicas, en la figura 5.20 observamos que Model 11 y Model 13 tienen una complejidad similar, a pesar de tener formas diferentes. En cuanto a las variaciones de Model 13, al que hemos ido reduciendo el número de ciclos con el mismo número de decisiones, representamos en la figura 5.22 los valores de complejidad de Model 13 y sus variaciones Model 18, Model 19, Model 20. Observamos que la complejidad aumenta linealmente con la misma tendencia a excepción del Model 20, donde ya no hay ciclos, ni tampoco caminos alternativos, como si ocurría en los otros modelos mostrados en esta figura. 


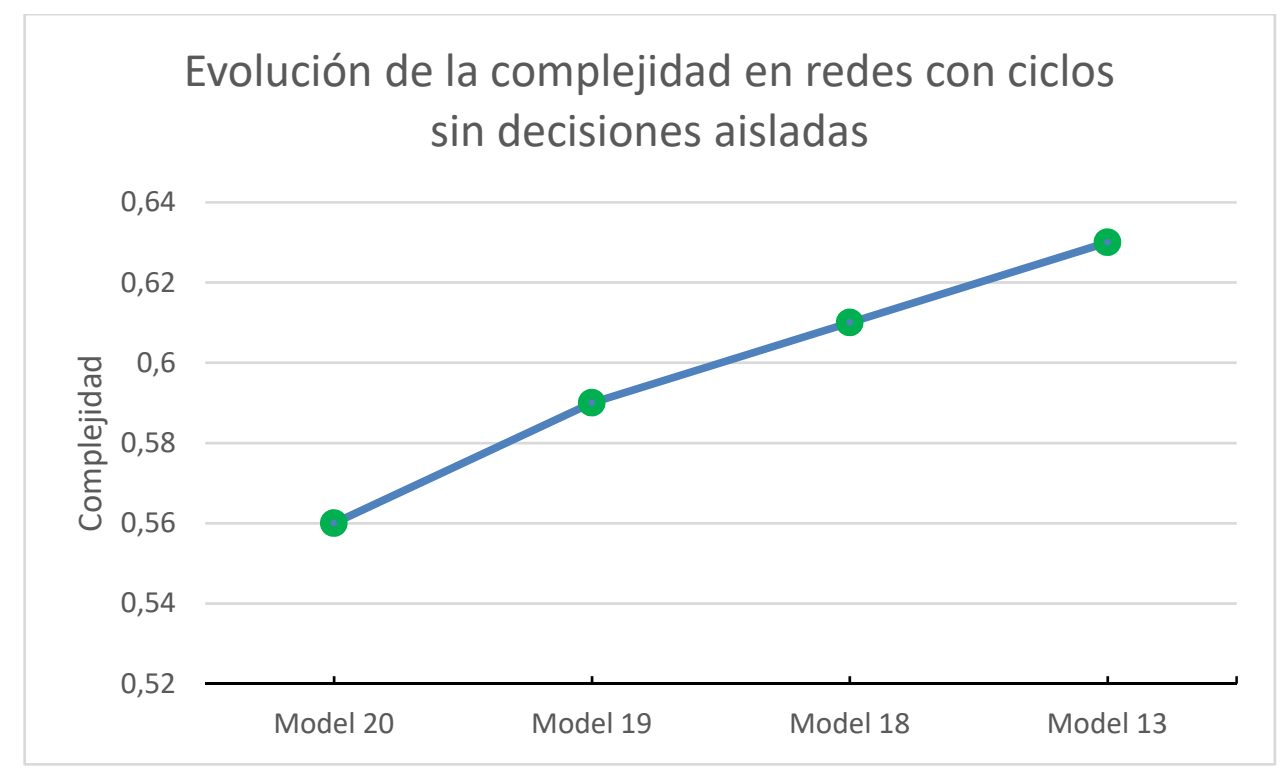

Figura 5.22. Evolución de complejidad en variantes de redes con topología con ciclos.

En cuanto a la validación de la métrica $A D D N-C$ con las redes $S B P 3$ y $S B P 4$ analizadas, podemos decir que redes con mayor número de decisiones y relaciones no tienen por qué tener una mayor complejidad.

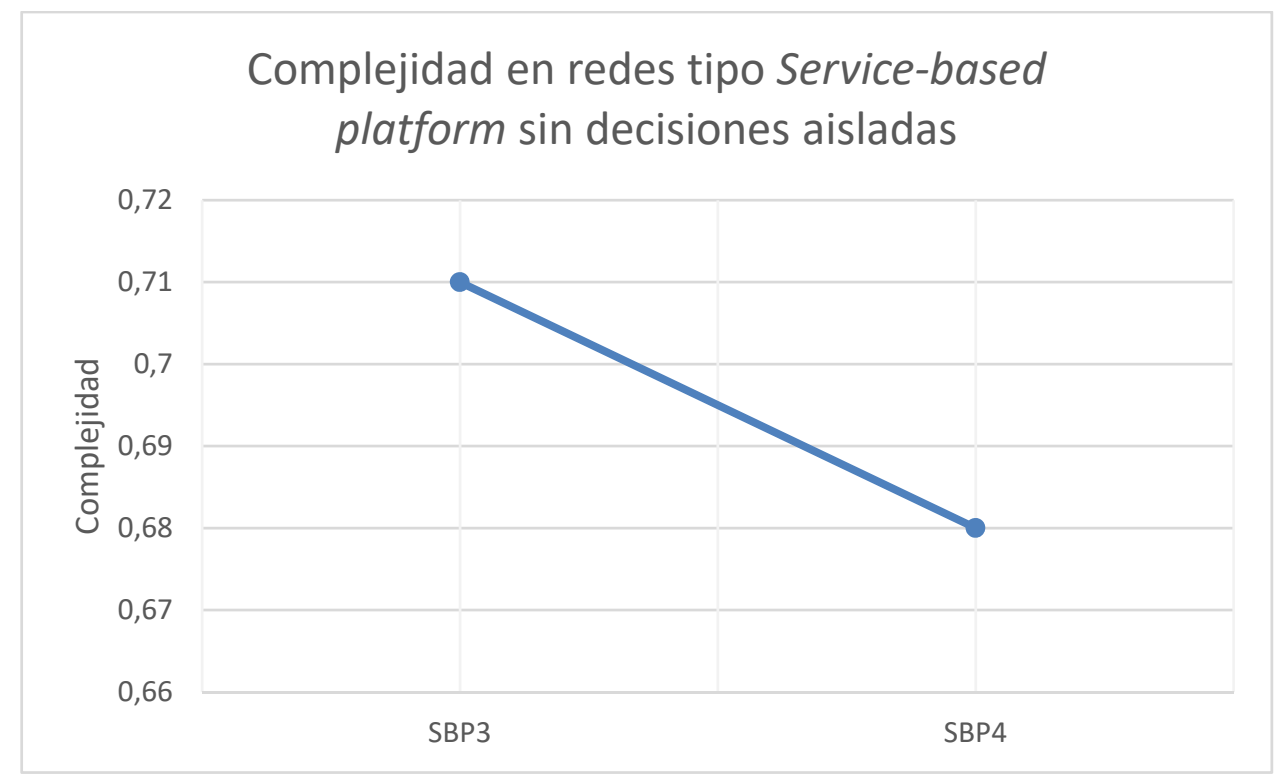

Figura 5.23. Complejidad en redes Service-based platform sin decisiones aisladas.

Como observamos en la figura 5.23, los valores de complejidad obtenidos para las redes $S B P 3$ y $S B P 4$, no son ni las más altas ni las más bajas de todas las calculadas.

Resultados de redes con decisiones aisladas: De los datos obtenidos en la tabla 5.2, generamos su representación gráfica de la figura 5.24, y en ella observamos: 


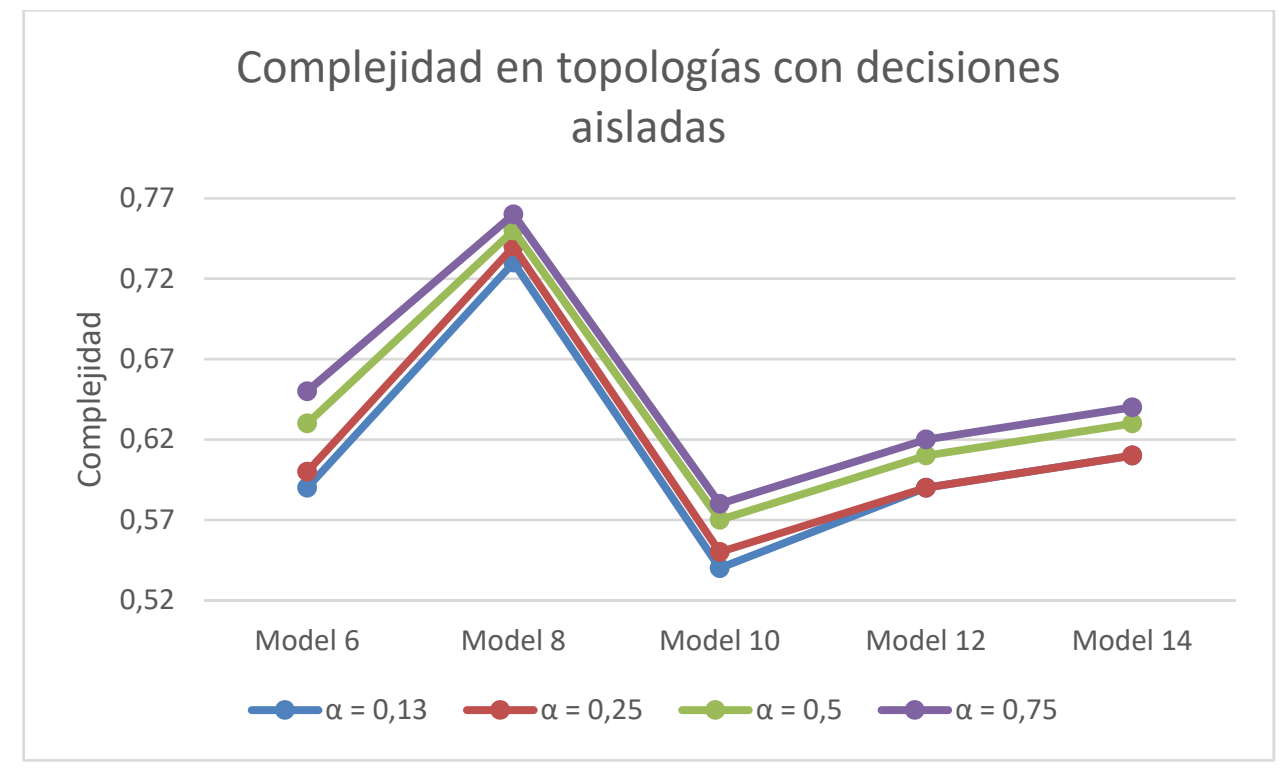

Figura 5.24. Complejidad en redes con decisiones aisladas.

A medida que aumentamos el factor de corrección de importancia de las decisiones aisladas, la complejidad aumenta en todos los casos.

La topología en árbol (Model 8) tiene una complejidad mayor que el resto de topologías. La incorporación de una decisión aislada no influye de manera apreciable a la complejidad resultante. En la figura 5.24 observamos que para los cuatro valores del factor de corrección de importancia $\alpha$ empleados, la complejidad es muy próxima para este modelo.

Observamos una notable diferencia de valores calculados para cada valor de $\alpha$, cuando la red tiene pocas decisiones y relaciones frente a redes con mayor número de dicesiones y trazas cuya la complejidad obtenida es muy próxima, e incluso coincidir en magnitud en redes con un elevado número de decisiones no aisladas (Model 14).

En los modelos con ciclos Model 10, Model 12 y Model 14, la complejidad obtenida con los cuatros valores del factor de corrección $\alpha$ es muy similar y asciende en función del número de elementos arquitectónicos que forman las topologías.

Al comparar la evolución de la complejidad respecto al valor de factor $\alpha$ (figura 5.25) observamos que todas tienen una ascendencia lineal con poca inclinación. Además, dicha pendiente es mayor en las redes con pocos elementos arquitectónicos (Model 6). En este tipo de topologías, la relación decisiones aisladas con respecto a las no aisladas es mayor que en el resto de topologías. 


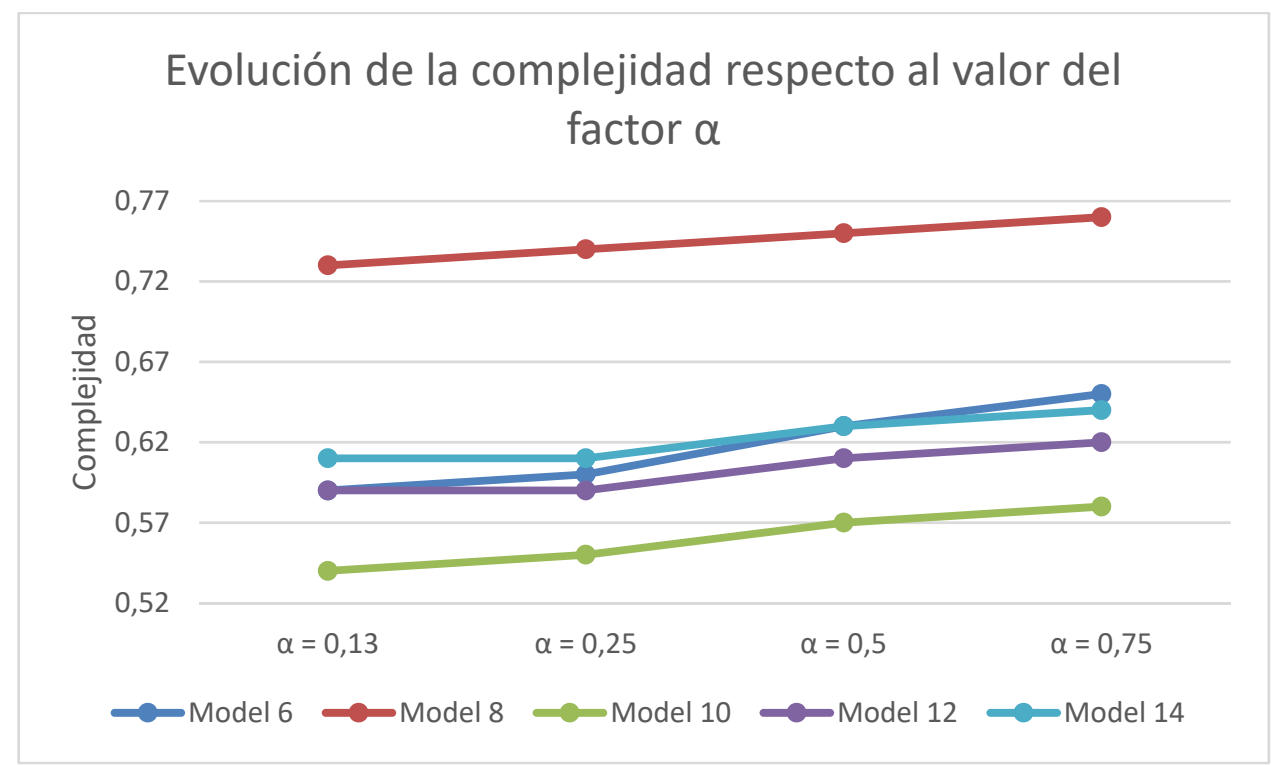

Figura 5.25. Evolución de la complejidad respecto al valor del factor $\alpha$.

En cuanto a las redes complejas analizadas, y cuyas gráficas se corresponde con las figuras 5.26 y 5.27, observamos que la complejidad está en relación a la topología de la red. A pesar que SBP1 y SBP3 tienen un número elevado de decisiones aisladas (40 aprox. cada una) la complejidad obtenida no es mucho mayor que la misma topología sin dichas decisiones aisladas.

En la figura 5.26, que representa la complejidad de SBP5 frente a los dos modelos que la forman, observamos que en todos los casos del factor $\alpha$, la complejidad de SBP5 se aproxima más a la de la red que más decisiones y relaciones aporta, que es $S B P 1$, el cual tiene prácticamente el doble de decisiones y relaciones que SBP2.

Para valores de $\alpha$ inferiores o iguales a 0,5, la complejidad presente en SBP5 es mayor respecto a las obtenidas para SBP1 y SBP2. Ambos modelos comparten prácticamente las mismas decisiones aisladas, y al fusionarse (SBP5) el número de decisiones aisladas no crece, no así el resto de decisiones y relaciones.

En el caso de $\alpha$ igual a 0,75 , observamos que la complejidad obtenida en SBP5 es inferior a la de SBP2. Esto es debido a que nuestra métrica ADDN-C pondera más la multidependencia entre decisiones en relación al tamaño de la red. 


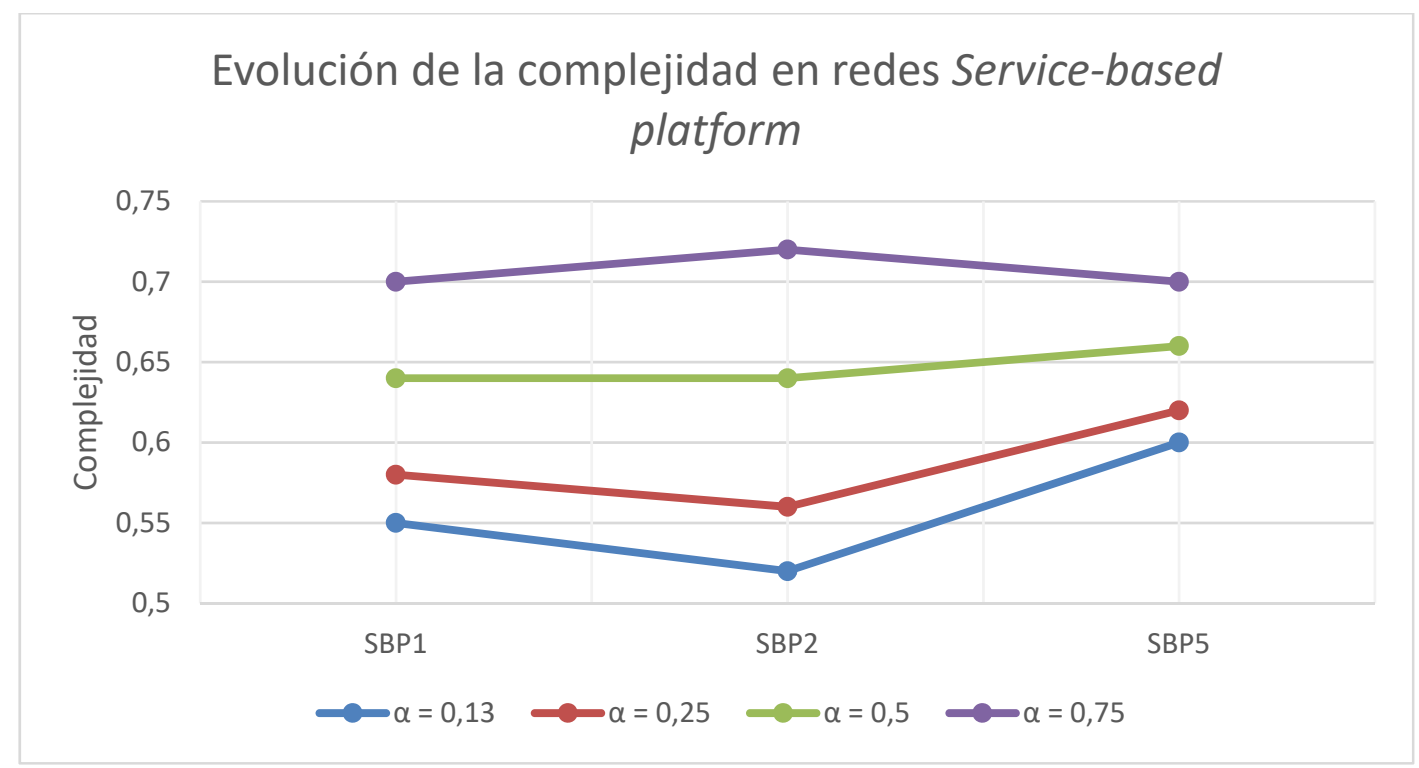

Figura 5.26. Evolución de la complejidad en redes Service-based platform respecto al factor $\alpha$.

En cuanto a la evolución de la complejidad con relación a los valores del factor $\alpha$ (figura 5.27), observamos que los modelos SBP1 y SBP5 tienden a aproximarse a medida que aumenta el valor de dicho factor. Sin embargo, la tendencia de complejidad de SBP2, que tiene aproximadamente un $50 \%$ de elementos arquitectónicos menos que SBP1, es mayor que la del resto de topologías. Cuando $\alpha$ es 0,5 tienen la misma complejidad y a partir de este valor, la complejidad de la red más pequeña supera a la de la red con mayor número de decisiones y relaciones.

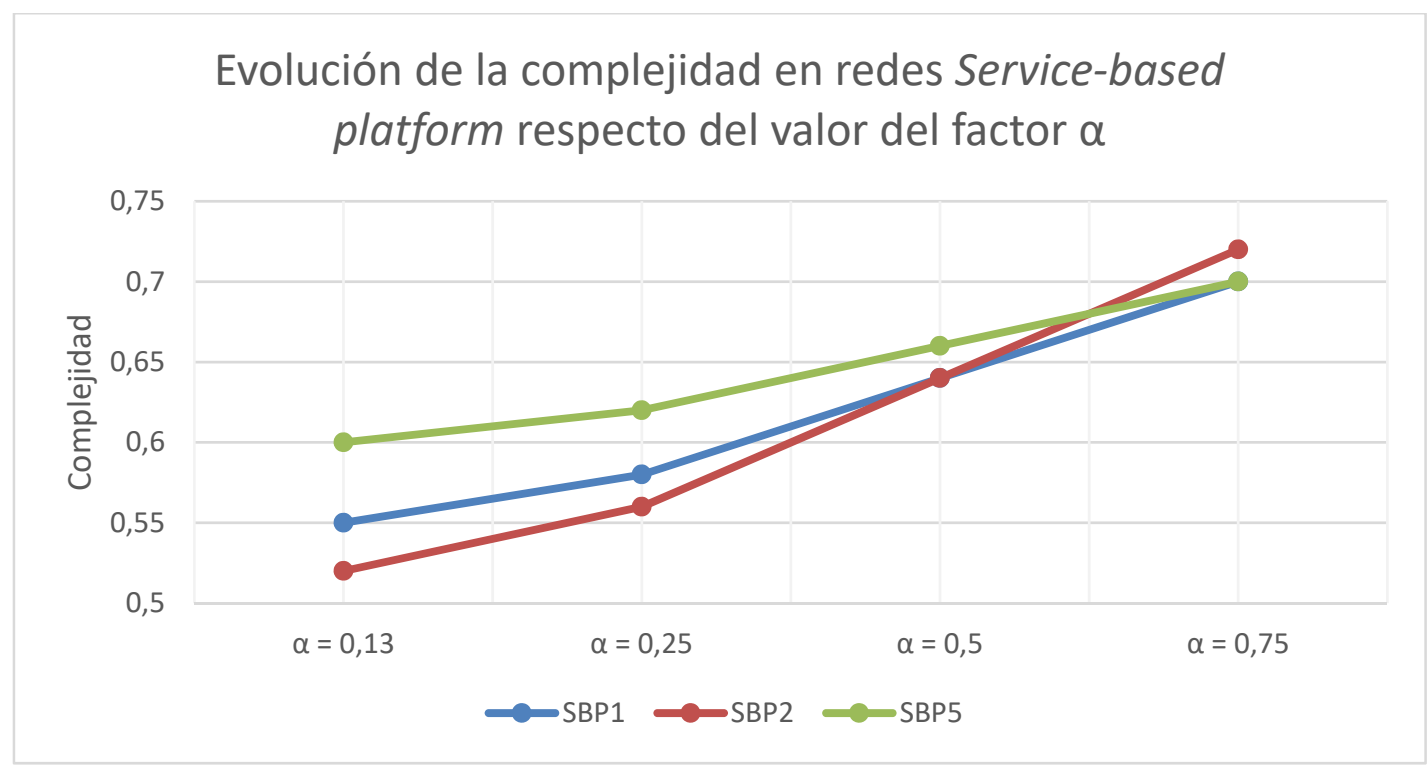

Figura 5.27. Tendencia de la complejidad con relación a la variación del factor $\alpha$ en redes Service-based platform. 
Recomendaciones para mejorar la sostenibilidad: La sostenibilidad del modelo de decisión tiende a ser peor cuando la complejidad aumenta. Pero esta complejidad puede disminuir cuando la topología cambia al reducir el número de dependencias entre las decisiones. Por lo tanto, mantener el número de decisiones controlado, en especial las decisiones alternativas, favorece una mejor mantenibilidad y por lo tanto una reducción de su complejidad. Otra forma de favorecer la sostenibilidad es reducir el número de ciclos en las topologías, como ya hemos indicado anteriormente.

\subsection{Sostenibilidad Basada en Costes}

En esta sección exponemos la experimentación realizada para validar la propuesta de métrica detallada en la sección 4.4.2. Para ello evaluaremos el esfuerzo requerido en capturar decisiones.

La validación de nuestra propuesta se basará en los datos obtenidos en un experimento donde grupos de arquitectos tendrán que especificar una arquitectura software a partir de los mismos requisitos. Con los datos ofrecidos, acorde a un modelo de captura de datos, procederemos a calcular el esfuerzo en la captura de decisiones según nuestra propuesta de métrica. A continuación, procederemos a analizar e interpretar los datos obtenidos.

\subsubsection{Experimento en la URJC}

Para evaluar nuestra propuesta de métrica de coste, proponemos un experimento con alumnos de la Universidad Rey Juan Carlos, de Madrid. Propondremos a 64 alumnos, divididos en 11 grupos, que contabilicen el tiempo empleado en diseñar una arquitectura software, cuyos requisitos serán los mismos para todos ellos. La duración de este experimento fue de 4 semanas, con una dedicación mínima de 2 horas/semana. Permitimos que los alumnos emplearán un mayor número de horas.

Además, en cada grupo diferenciamos tres tipos de arquitectos software con diferentes roles. Cada grupo estará formado por dos miembros para capturar decisiones (arquitectos seniors), otro dos reflexionarán (arquitectos cognitivos) si cada decisión es idónea, está motivada y relacionada con requisitos y/o con decisiones ya tomadas. La existencia de arquitectos seniors y cognitivos está basado en la aplicación del método "Mind 1 and 
Mind 2" 13. El resto del grupo modelará la arquitectura (arquitectos juniors) a partir de las decisiones ya capturadas, y no participan en la toma y reflexión de decisiones. A todos los grupos se les proporciona un enunciado con los requisitos, y tres plantillas para capturar 7 (tabla 5.5), 10 (tabla 5.6) y 14 (tabla 5.7) atributos por cada decisión. Cada grupo puede elegir un modelo de estas tres plantillas.

La especificación de la arquitectura software la detallamos en el Anexo VI. Además, a modo de ejemplo proporcionamos una plantilla completada con los atributos de una decisión. Para modelar la arquitectura, permitimos un modelado UML o mediante la herramienta ADMentor ${ }^{14}$.

Una vez finalizado el experimento procedemos a analizar los datos proporcionados. A modo de ejemplo destacamos en la tabla 5.5 una decisión de diseño capturada por uno de los grupos participantes en este experimento. En ella observamos con detalle el contenido de cada uno de los atributos capturados, teniendo en cuenta que en esta plantilla se corresponde con el formato "Long". En el campo "Alternative decisions", los arquitectos seniors identifican las tres decisiones alternativas que plantean en relación a esta decisión. Además, como se puede observar, se identifican los requisitos relacionados con esta decisión, así como el identificador de sendas figuras correspondientes a su modelado arquitectónico.

13 El método "Mind 1 and Mind 2", descrito por Razavian et al. [RAZA 16], contempla que un arquitecto (senior) toma decisiones motivadas y otro (cognitivo) reflexiona sobre la idoneidad de la decisión tomada por el primero, en base al cumplimiento de requisitos funcionales y de calidad o de las decisiones ya tomadas.

${ }^{14}$ La herramienta ADMentor (Architectural Decision Modeling Add-In for Sparx Enterprise Architect) permite el modelado de la arquitectura software. Ha sido creada por el Institute for Software, University for Applied Science, Switzerland.'La herramienta está disponible en la URL https://www.ifs.hsr.ch/ADMentor-Tool.13201.0.html?\&L=4 
Capítulo 5. Experimentación

Tabla 5.5. Decisión de diseño capturada

\begin{tabular}{|c|c|c|}
\hline System: SCE & ID: DD-01 & Date: $18 / 10 / 2016$ \\
\hline Name: & \multicolumn{2}{|c|}{ Uso de un estilo arquitectónico combinando EVENT DRIVEN SOA (SOA 2.0). } \\
\hline Description & \multicolumn{2}{|c|}{$\begin{array}{l}\text { Esta decisión separa el sistema en } 3 \text { capas: una capa para la captación de } \\
\text { eventos, otra para el procesador de eventos, y otra para consumir los eventos, } \\
\text { en esta última hay una capa de servicios }\end{array}$} \\
\hline Rationale & \multicolumn{2}{|c|}{$\begin{array}{l}\text { Tomamos esta decisión porque queremos que algunos eventos produzcan } \\
\text { unos servicios que serán consumidos por terceros }\end{array}$} \\
\hline Pros & \multicolumn{2}{|c|}{$\begin{array}{l}\text { Podemos gestionar el sistema con modularidad, acoplamiento abierto, } \\
\text { separación de elementos de interés, reutilización y compatibilidad. }\end{array}$} \\
\hline Cons & \multicolumn{2}{|c|}{ No se encuentra en uso por empresas con este fin } \\
\hline Status: & \multicolumn{2}{|l|}{ Aprobada } \\
\hline Responsible: & \multicolumn{2}{|c|}{$\begin{array}{l}\text { - } \text { Arquitecto } 1 \\
\text { - } \text { Arquitecto } 2\end{array}$} \\
\hline \multicolumn{2}{|c|}{ Alternative decisions } & $\begin{array}{l}\text { - DD-01.1: MVC } \\
\text { - DD-01.2: SBA } \\
\text { - DD-01.3: SOA+EDA }\end{array}$ \\
\hline \multicolumn{2}{|c|}{ Related to requirements } & $\begin{array}{ll}- & \mathrm{RF} 2.1 \\
- & \mathrm{RF} 4.0 \\
0 & \mathrm{RF} 4.1 \\
-\quad & \mathrm{RF} 5.0 \\
0 \quad \mathrm{RF} 5.4 \\
\quad \mathrm{RF} 5.4 .1 \\
\end{array}$ \\
\hline \multicolumn{2}{|c|}{$\begin{array}{l}\text { Related to software } \\
\text { artifacts }\end{array}$} & $\begin{array}{l}\text { - DAdMentor_DD01.bmp } \\
\text { - DPaquetes_DD01.bmp }\end{array}$ \\
\hline \multicolumn{3}{|c|}{ Constraints } \\
\hline \multicolumn{2}{|c|}{ Related to decisions } & \\
\hline
\end{tabular}

A continuación, señalamos una decisión alternativa de DD01, y representada en la tabla 5.5, tal y como se indica "Alternative decisions"

Tabla 5.6. Decisión de diseño alternativa capturada

\begin{tabular}{|c|c|c|c|}
\hline System: SCE & \multicolumn{2}{|c|}{ ID: DD-01.1 } & Date: $18 / 10 / 2016$ \\
\hline Name: Sele & \multicolumn{3}{|c|}{ Selección del estilo arquitectónico MVC } \\
\hline Description & \multicolumn{3}{|c|}{$\begin{array}{l}\text { Esta decisión consistirá en el uso de un MVC para gestionar la entrada de los } \\
\text { eventos en el sistema cuando se produce una petición y el controlador } \\
\text { responderá a estos eventos }\end{array}$} \\
\hline Rationale & \multicolumn{3}{|c|}{ Tomamos la decisión para poder gestionar las diferentes peticiones } \\
\hline Pros & \multicolumn{3}{|c|}{ Fácil integración y separación de funcionalidades } \\
\hline Cons & \multicolumn{3}{|c|}{ Poco útil frente a eventos simultáneos y riego de desborde } \\
\hline Status: & \multicolumn{3}{|c|}{ Rechazada } \\
\hline Responsible: & \multicolumn{3}{|c|}{$\begin{array}{l}\text { - } \text { Arquitecto } 3 \\
\text { - } \text { Arquitecto } 4\end{array}$} \\
\hline \multicolumn{2}{|c|}{ Alternative decisions } & $\begin{array}{ll}\bullet \\
\bullet \\
\bullet\end{array}$ & $\begin{array}{l}-01: \text { SOA } 2.0 \\
-01.2: \text { SBA } \\
-01.3: \text { SOA+EDA }\end{array}$ \\
\hline
\end{tabular}


En la figura 5.28 representamos la decisión de diseño capturada (tabla 5.5) y cuya imagen ha sido generada por la herramienta ADMentor ${ }^{15}$.

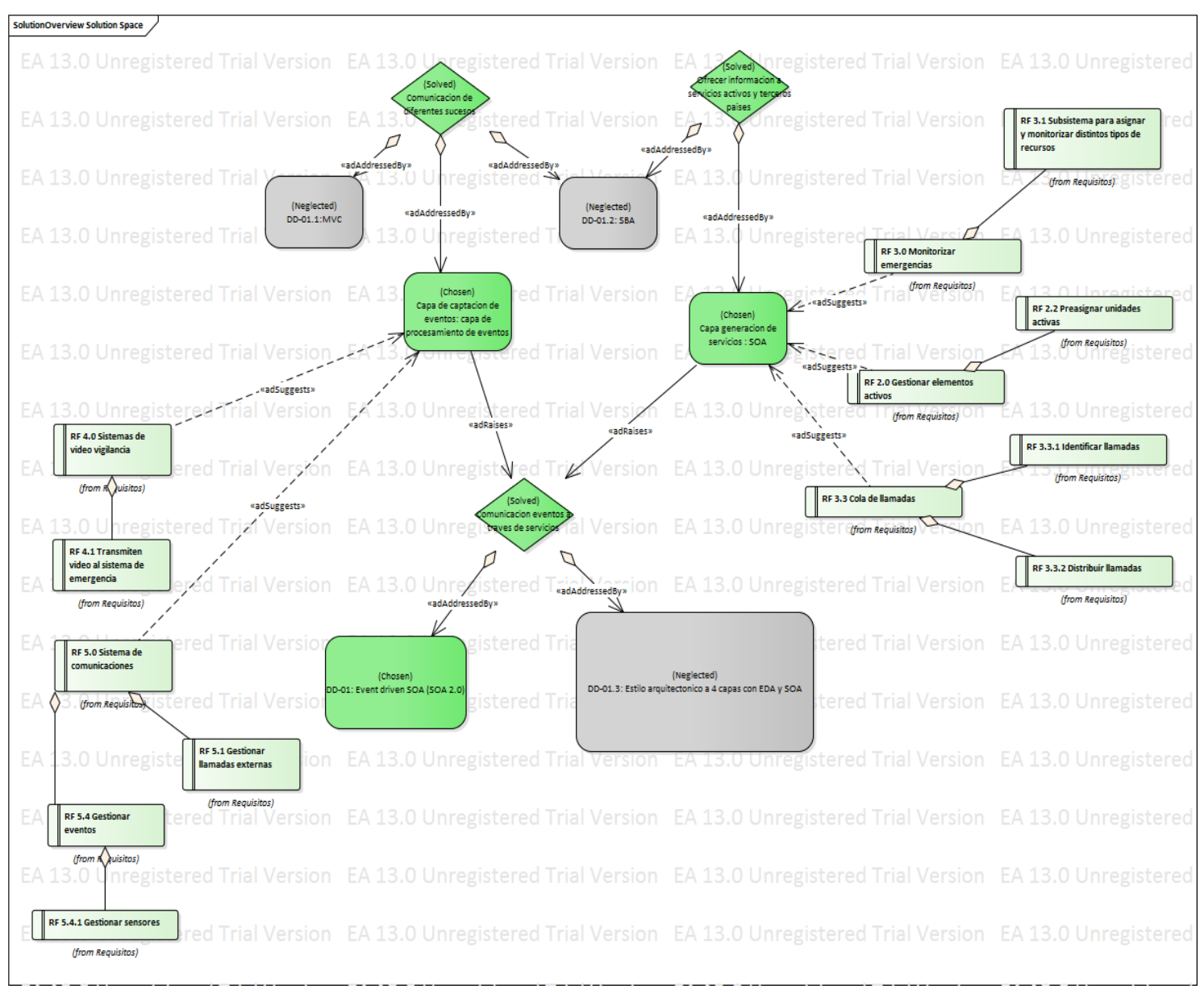

Figura 5.28. Representación gráfica de la decisión de diseño DD01 capturada

\subsubsection{Evaluación}

Una vez finalizado este experimento, observamos que dos de los grupos proporcionan información no valida y decidimos excluirlos del experimento. En concreto el grupo G9 no finaliza el experimento y el grupo G8, a pesar de finalizar, no detalla que tipo de plantilla eligió.

Con el fin de evaluar el impacto del coste en la sostenibilidad del proceso de toma de decisiones hemos definido un conjunto de preguntas, que deberán ser resueltas a partir de los datos obtenidos. Estas preguntas son:

\footnotetext{
${ }^{15} \mathrm{La}$ herramienta ADMentor permite capturar las decisiones y modelar la arquitectura software. La herramienta está accesible en: https://www.ifs.hsr.ch/index.php?id=13201\&L=4, https://github.com/IFSHSR/ADMentor
} 
RQ1. ¿Cuál es el número de atributos que resulta más sostenible para capturar decisiones de diseño?

RQ2. ¿Cuál es el número de alternativas por decisión que requiere, por término medio, menor esfuerzo?

RQ3. ¿Cuál es la relación de incremento de esfuerzo debido al uso del modelo Mind1Mind2?

\subsubsection{Resultados}

A continuación, detallamos los datos obtenidos que nos permiten contestar cada una de las preguntas planteadas anteriormente.

RQ1. ¿Cuál es el número de atributos que resulta más sostenible para capturar decisiones de diseño?

En la tabla 5.7 reflejamos los datos ofrecidos por los diferentes grupos vinculados a esta pregunta, como son las decisiones capturadas por cada grupo, el tiempo empleado por los arquitectos seniors al capturar dichas decisiones, así como el modelo de plantilla elegido por cada grupo para capturar las decisiones. A partir de estos valores calculamos la estimación de esfuerzo obtenidos por nuestra métrica $A D D N-E$.

Tabla 5.7. Esfuerzo para capturar decisiones según la plantilla empleada.

\begin{tabular}{|c|c|c|c|c|}
\hline & ADD & $\begin{array}{c}\text { Tiempo } \\
\text { empleado }\end{array}$ & Plantilla & $\begin{array}{r}\text { Esfuerzo } \\
\text { ADDN-E }\end{array}$ \\
\hline G1 & 45 & 11,83 & Short & 26,62 \\
\hline G2 & 11 & 3,02 & Long & 51,05 \\
\hline G3 & 15 & 22,17 & Medium & 6,77 \\
\hline G4 & 14 & 3 & Medium & 46,67 \\
\hline G5 & 21 & 8,75 & Long & 33,60 \\
\hline G6 & 19 & 4,75 & Medium & 40,00 \\
\hline G7 & 14 & 7,08 & Short & 13,84 \\
\hline G10 & 14 & 7,67 & Short & 12,78 \\
\hline G11 & 9 & 32,92 & Medium & 2,73 \\
\hline
\end{tabular}


A partir de los valores reflejados en la tabla 5.7, generamos la gráfica 5.29 para representar el esfuerzo de capturar decisiones (columnas de color morado) en aquellos grupos que han utilizado la plantilla Short (línea color naranja), Medium (línea color rojo) y Long (línea color verde).

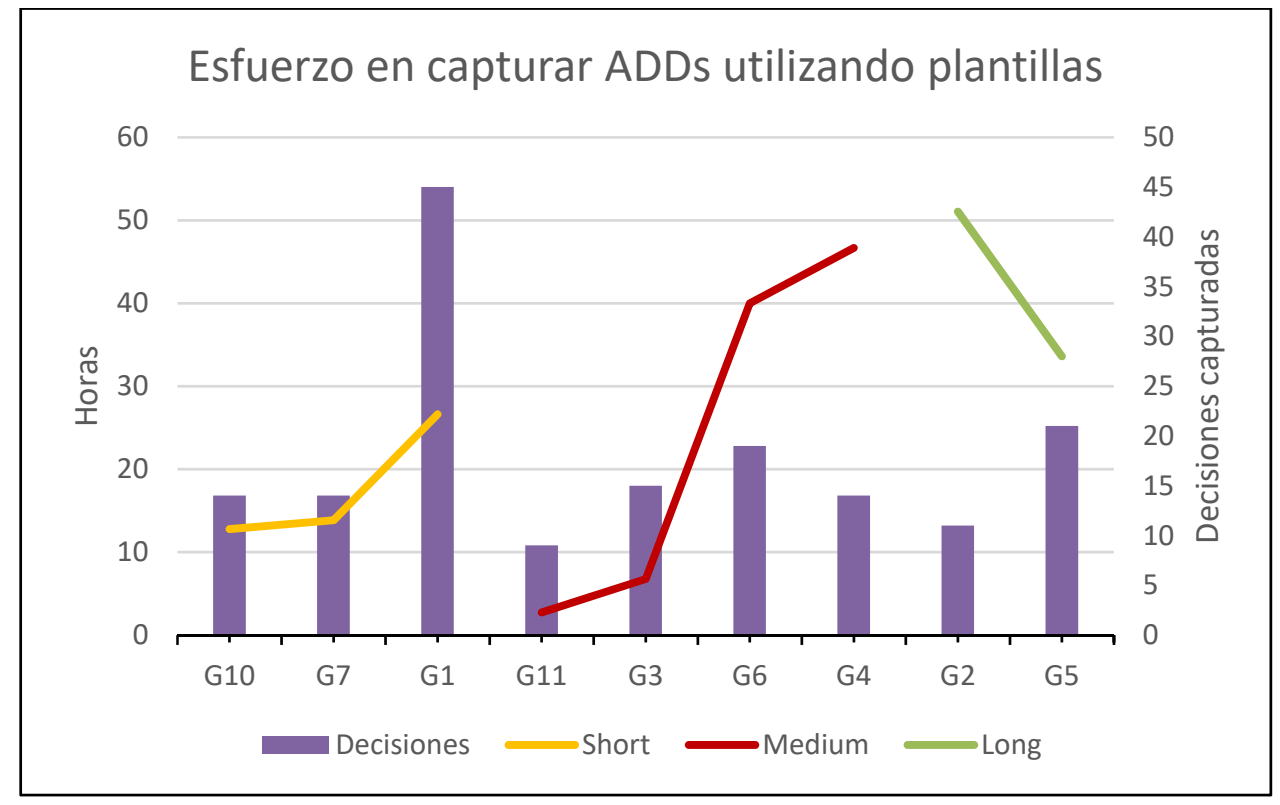

Figura 5.29. Esfuerzo realizado en capturar ADDs utilizando diferentes plantillas

De los datos obtenidos al usar la plantilla Short (7 atributos), podemos afirmar que el esfuerzo requerido para capturar las decisiones es creciente al número de decisiones capturadas. Los grupos G10 y G7 capturan el mismo número de decisiones con un ligerísimo esfuerzo mayor por parte de G7. El grupo G1, que triplica en número de decisiones capturadas los grupos G10 y G7, les duplica en esfuerzo a estos dos grupos.

Con el uso de la plantilla Medium con 10 atributos, también podemos decir que el esfuerzo en capturar crece a medida que aumenta el número de decisiones, a excepción del grupo G4 que, a pesar de capturar menos decisiones que G3 y G6, ha requerido un esfuerzo mayor, incluso al esfuerzo requeridos por algún grupo que optó por utilizar la plantilla Long. Creemos que el comportamiento del grupo G4 es debido a la aportación de respuestas incorrectas por parte de los miembros del equipo.

De hecho, con los datos facilitados con el uso de esta plantilla observamos la mayor divergencia de valores obtenidos y calculados. El grupo G3 captura un número de decisiones similar a los grupos G10 y G7, ambos con plantilla Short, pero el esfuerzo requerido es prácticamente la mitad al requerido por dichos grupos. Si comparamos los resultados obtenidos por el grupo G4, con el mismo número de decisiones que G10 y G7, 
el esfuerzo requerido por G4 triplica al de dichos grupos. Por último, podemos establecer un razonamiento análogo entre los grupos G11 con G10 y G7.

Por último, los grupos que optaron por la plantilla Long con 14 atributos, tienen un comportamiento divergente, ya que de ella no podemos confirmar que el esfuerzo en capturar decisiones es proporcional al número de dichas decisiones, y por ello contradice los razonamientos que hemos formulado anteriormente en relación al esfuerzo requerido para capturar decisiones. Consideramos que este comportamiento se deba a respuestas incorrectas o incompletas de los miembros de alguno de los dos equipos, como ocurre con el grupo G5 que capturó decisiones, pero sólo determinó de manera parcial el tiempo empleado. En cualquier caso, los dos grupos G2 y G5 han realizado un esfuerzo mayor que el resto de grupos que han terminado este experimente, con la salvedad de los G4 y G6.

Además, debemos tener en cuenta la experiencia de los arquitectos. En este sentido destacamos el elevado número de decisiones capturadas por el grupo $\mathrm{G} 1$, respecto al resto de grupos, a pesar que los requisitos son los mismos para todos los grupos. También destacamos que el grupo G2 captura prácticamente la mitad de decisiones que el grupo G5 con un tercio más de esfuerzo.

Con los datos obtenidos, no podemos concluir que número de atributos resulta más sostenible para capturar las decisiones de diseño, pero si podemos afirmar que las plantillas Short y Medium requieren por término medio menos esfuerzo

RQ2. ¿Cuál es el número de alternativas por decisión que requiere, por término medio, menor esfuerzo?

A todos los grupos les solicitamos que nos indicaran el tiempo empleado en capturar 1 decisión y dos alternativas, 1 decisión y entre 2 y 4 alternativas y 1 decisión y más de 4 alternativas. Los datos facilitados por grupo los reflejamos en la tabla 5.8. 
Tabla 5.8. Tiempo (en horas) requerido para capturar 1 decisión con múltiples alternativas.

\begin{tabular}{|c|c|c|c|}
\hline & $\begin{array}{c}\text { 1 ADD y } \\
\text { 2 alternativas }\end{array}$ & $\begin{array}{c}\text { 1 ADD y } \\
\text { 2-4 alternativas }\end{array}$ & $\begin{array}{c}\text { 1 ADD y } \\
\text { > } \text { alternativas }\end{array}$ \\
\hline G1 & 0,25 & 0,50 & 0,50 \\
\hline G2 & 3,17 & 4,67 & 4,00 \\
\hline G3 & 0,75 & 1,83 & 2,50 \\
\hline G4 & 0,92 & 1,08 & 1,17 \\
\hline G5 & 1,17 & 1,33 & --- \\
\hline G6 & 1,50 & 1,75 & 8,50 \\
\hline G7 & 2,17 & 3,00 & 4,00 \\
\hline G10 & 2,00 & 3,00 & 2,00 \\
\hline G11 & 3,17 & 4,58 & 7,50 \\
\hline
\end{tabular}

Hay que resaltar que el grupo G5 declara que no llegó a capturar 1 decisión con cuatro alternativas. Estos datos los visualizamos en la figura 5.30, y en ella observamos que todos los grupos han requerido mayor tiempo en capturar entre 2 y 4 alternativas con respecto al tiempo empleado en capturar 2 alternativas. Excluyendo al grupo G5, que declaró no haber capturado más de 4 alternativas, sólo los grupos G2 y G10 han empleado menor tiempo en capturar más de 4 alternativas.

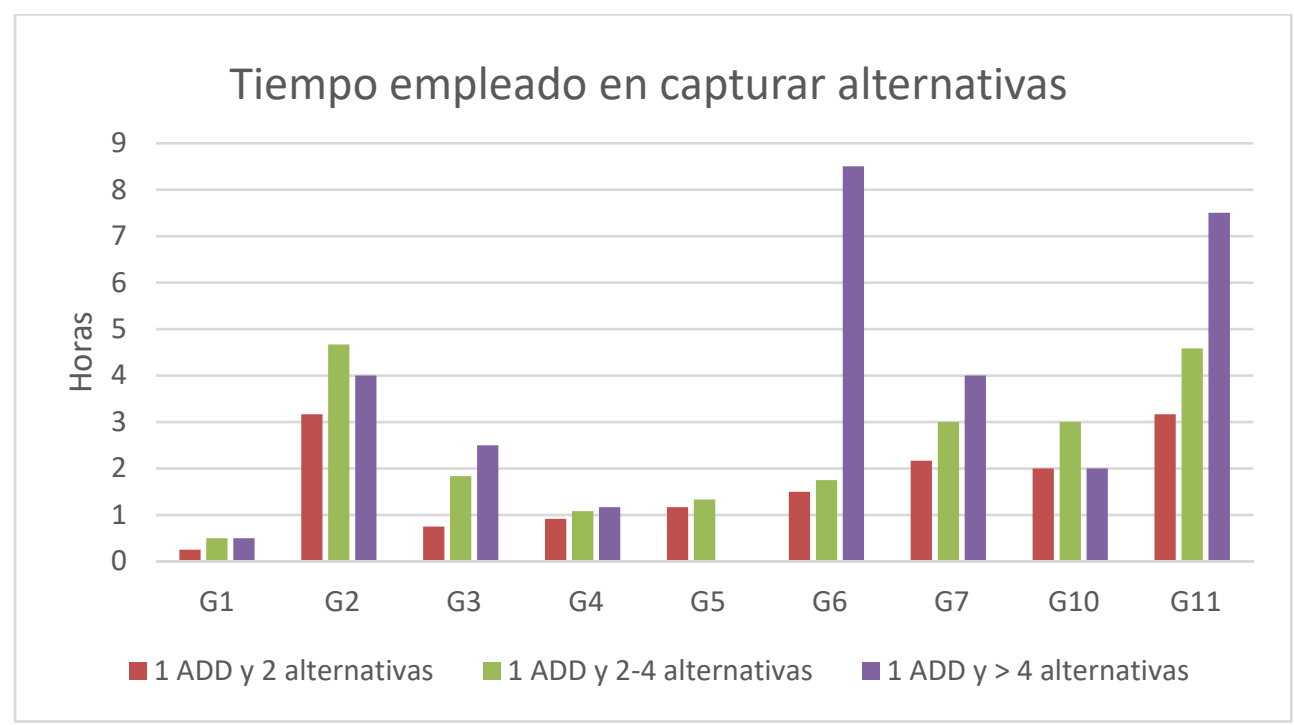

Figura 5.30. Tiempo (en horas) empleado por cada grupo en capturar alternativas de diseño. 
Con los datos que hemos reflejado en la tabla 5.8, calculamos el esfuerzo total en horas de todos los grupos. A continuación, promediamos ese tiempo en relación al número medio de alternativas, con el fin de poder responder a nuestra pregunta RQ2. En el caso del tiempo requerido para capturar entre 2 y 4 alternativas, promediamos este tiempo entre 3 alternativas. Para el caso de más de 4 alternativas, analizamos los datos facilitados por todos los grupos y consideramos que 5 es el promedio de alternativas capturadas por todos los grupos. Con este promedio queremos determinar el tiempo requerido en capturar una alternativa, una vez que el arquitecto ha captura decisiones. Estos datos los mostramos en la tabla 5.9 .

Tabla 5.9. Tiempo (horas) medio estimado para capturar decisiones con múltiples alternativas.

\begin{tabular}{|l|c|c|c|} 
& $\begin{array}{c}\text { 1 ADD y } \\
\text { 2 alternativas }\end{array}$ & $\begin{array}{c}\text { 1 ADD y } \\
\text { 2-4 alternativas }\end{array}$ & $\begin{array}{c}\text { 1 ADD y } \\
\text { 4 alternativas }\end{array}$ \\
\hline Tiempo total & 15.08 & 21,75 & 30,17 \\
\hline $\begin{array}{l}\text { Tiempo medio } \\
\text { / alternativas }\end{array}$ & 7.54 & 7.25 & 6.03 \\
\hline
\end{tabular}

A partir de los valores representados en la tabla 5.9 generamos la figura 5.31.

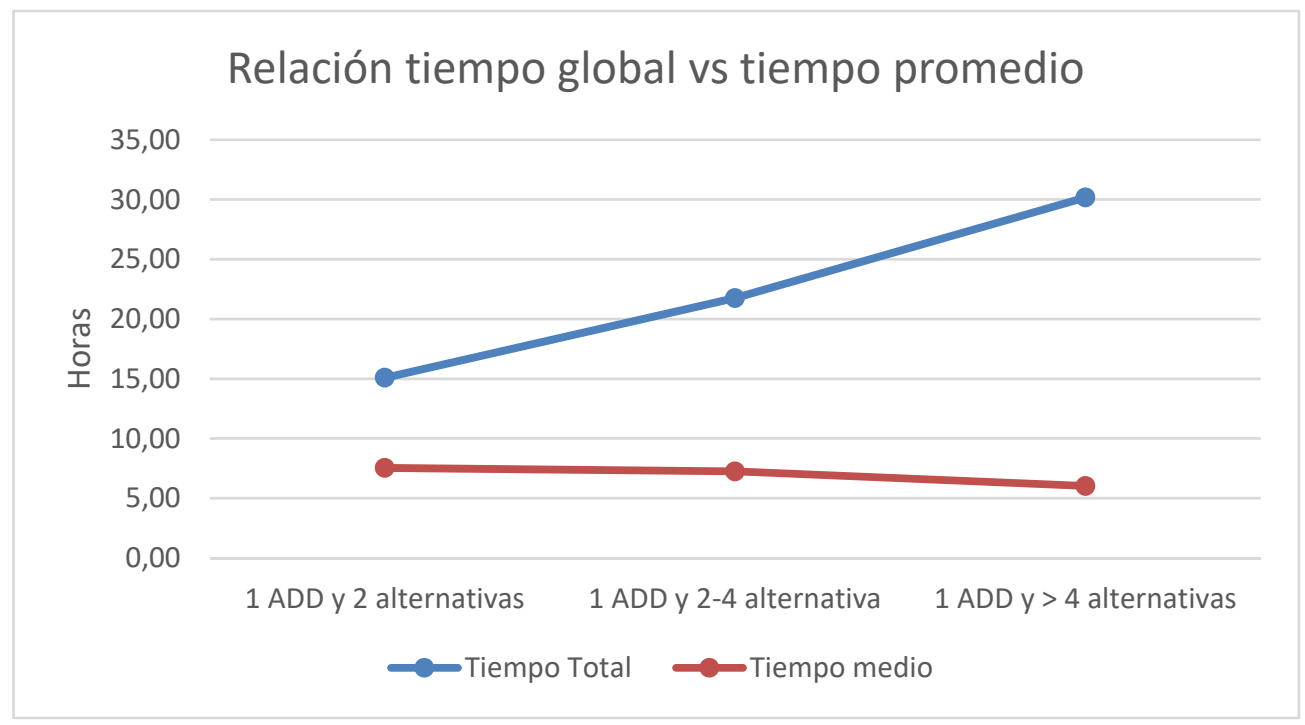

Figura 5.31. Relación de tiempo global y tiempo promedio en capturar una decisión y varias alternativas.

En ella observamos que el tiempo total empleado en capturar diferentes alternativas (línea azul) es proporcional al número de las alternativas capturadas. Sin embargo, observamos un leve descenso en el tiempo medio (línea roja) a medida que crece el 
número de alternativas. Es decir, según los datos obtenidos el promedio en capturar 1 decisión y más de 4 alternativas requiere menor esfuerzo por término medio que capturar menos alternativas. Este dato contradice la lógica que a mayor captura de decisiones mayor es el tiempo requerido. Pero como ya hemos manifestado anteriormente, los valores aquí mostrados y analizados son los valores facilitados por los grupos participantes en el experimento. Hemos detectado ciertas anomalías en los datos facilitados por algunos de los grupos entrevistados. Por ejemplo, el esfuerzo de los grupos G6 y G11 para la realización de ciertas actividades es muy alto con respecto la tendencia observada en el resto de grupos. Además, hemos detectado inconsistencia de datos aportados por el grupo G5, que reportó haber capturado más de 4 alternativas, pero no aportó el tiempo requerido en su captura.

Cuando se procede a capturar más de 4 alternativas, el tiempo total requerido duplica el tiempo empleado para capturar sólo dos decisiones alternativas. Por lo tanto, si aumentamos el número de decisiones alternativas a capturar, la tendencia del gráfico mostrará una tendencia ascendente exponencial teniendo en cuenta el tiempo para hacer, deliberar y capturar más decisiones alternativas, lo que puede tener un claro impacto en proyectos ágiles. Como conclusión podemos decir que el esfuerzo total en capturar alternativas es proporcional al número de ellas.

\section{RQ3. ¿Cuál es el incremento de esfuerzo debido al uso del modelo Mind1-Mind2?}

Con esta pregunta queremos conocer qué incremento de esfuerzo se produce al capturar decisiones cuando coexisten arquitectos senior y cognitivos según el modelo Mind1-Mind2, concretamente el introducido por los arquitectos cognitivos. En la tabla 5.10 mostramos los datos facilitados por cada grupo de arquitectos que han participado en nuestro experimento, y el incremento atribuidos en la actividad de los arquitectos cognitivos. 
Tabla 5.10. Incremento del esfuerzo (en horas) debido a la actividad de los arquitectos cognitivos, según el modelo Mind1-Mind2.

\begin{tabular}{|c|c|c|c|}
\hline & \multicolumn{2}{|c|}{ Esfuerzo } & Incremento (\%) por \\
\hline Grupo & Senior & Cognitivo & \begin{tabular}{c} 
esfuerzo cognitivo \\
\hline G1
\end{tabular} \\
\hline G2 & 31,83 & 5,58 & 32,06 \\
\hline G3 & 22,17 & 9,08 & 47,38 \\
\hline G4 & 3,00 & 2,58 & 46,07 \\
\hline G5 & 8,75 & 3,67 & 29,53 \\
\hline G6 & 4,75 & 2,97 & 38,44 \\
\hline G7 & 7,08 & 4,58 & 39,29 \\
\hline G10 & 7,67 & 2,00 & 20,69 \\
\hline G11 & 32,92 & 12,33 & 27,26 \\
\hline Media & 11,24 & 5,06 & 34,44 \\
\hline
\end{tabular}

Por los datos facilitados observamos que el esfuerzo empleado por los arquitectos cognitivos es inferior al tiempo empleado por los arquitectos seniors en capturar decisiones, como por otra parte es lógico. De los datos facilitados, deducimos que la actividad del arquitecto cognitivo representa un $34.44 \%$ del esfuerzo en la toma de captura de decisiones, por término medio. Observamos que el grupo G11 ha requerido un mayor esfuerzo en capturar decisiones y en reflexionar, tal vez debido a la inexperiencia de los miembros que lo forman. De manera análoga podemos razonar para el grupo G3, pero en este caso los valores no son tan altos.

En la figura 5.32 representamos estos datos, donde el eje izquierdo representa el incremento porcentual introducido por los arquitectos cognitivos en el proceso de captura de decisiones y el eje derecho, el esfuerzo en horas empleando en reflexionar sobre las decisiones capturadas. 


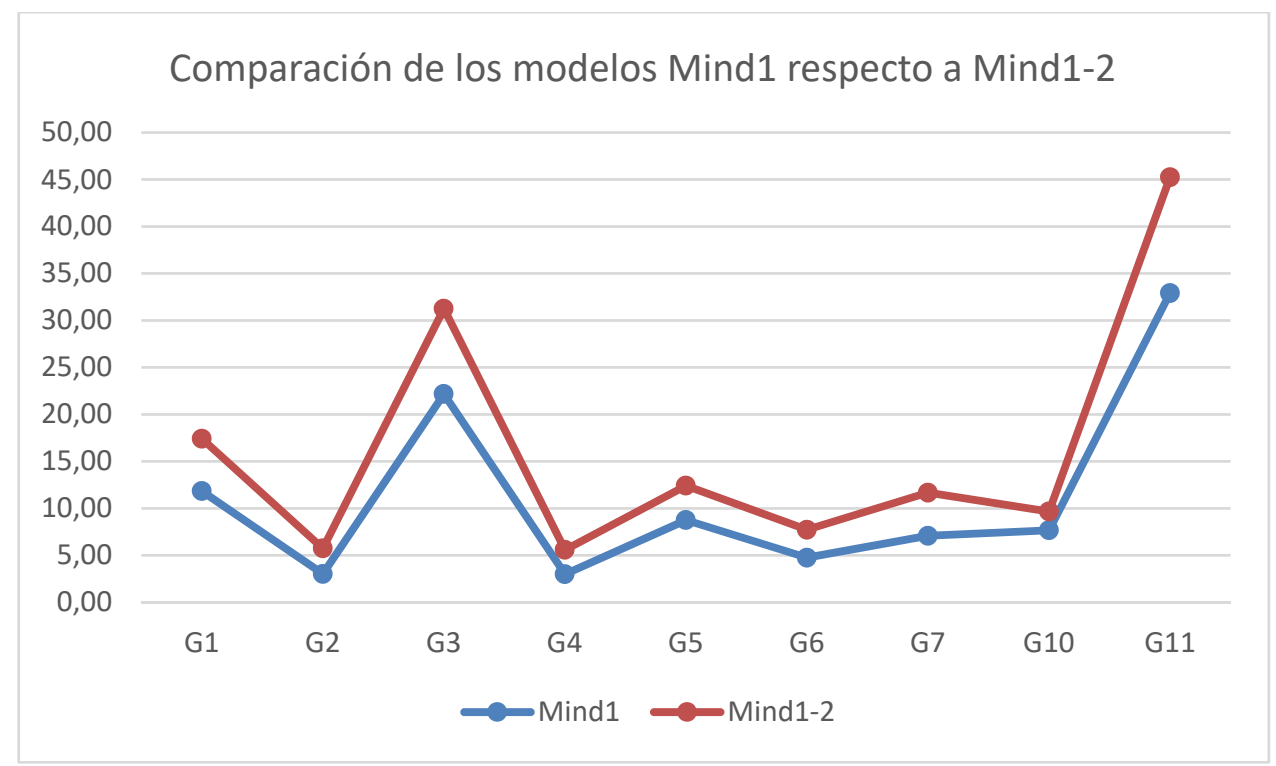

Figura 5.32. Incremento de esfuerzo utilizando el modelo Mind 1-2

De los datos obtenidos observamos que los grupos G3 y G11 son los dos únicos grupos cuyos esfuerzos están por encima del 35\%. Sin embargo, sus incrementos porcentuales de esfuerzo, debido a la actividad cognitiva, están por debajo de la media, y prácticamente son los más bajos con la excepción del grupo G10, cuyo esfuerzo cognitivo ha sido el más bajo de todos. Por término medio, el arquitecto cognitivo ha incrementado el esfuerzo del grupo en alrededor de un 35\%. Sin embargo, cuatro grupos han requerido un esfuerzo cognitivo mayor que la media. Como conclusión podemos decir que la captura de decisiones de diseño basados en el modelo Mind1-2 requiere un coste adicional comparado con la toma de decisiones sin reflexión. Sin embargo, es posible que, de cara a la estabilidad y longevidad de las decisiones, el modelo Mind1-2 produzca decisiones de mayor calidad.

\subsection{Sostenibilidad Basada en la Evolución}

A continuación, detallaremos el proceso seguido para validar la propuesta de proceso de análisis de impacto debido a un cambio en una decisión descrito en la sección 4.4.3. Hemos ampliado la aplicación Architectural Design Decision Network Metrics (ADDNMetrics) para integrar la métrica $A D D N-I$. En el Anexo III detallaremos el diseño de esta aplicación y en el Anexo IV su implementación. 


\subsubsection{Redes basadas en plataformas de servicio}

Para la validación de esta métrica utilizamos la variante de la red de decisiones SBP3 representada en la figura 5.11 de la sección 5.1.3. Dado que la red es de tipo QOC, hemos eliminado las cuestiones asociadas a cada decisión al no considerarlas técnicamente una decisión. En cuanto a las respuestas a estas preguntas, las consideramos decisiones alternativas a la decisión asociada. Por ello, hemos "puenteado" una decisión con las respuestas a las ya mencionadas preguntas. En la figura 6.32 mostramos esta red de decisiones adaptada, que la identificamos como SBP6, en la que conservamos las relaciones de "Option", "Solution”, “Trigger_Decision” y también "Imply_Pattern”.

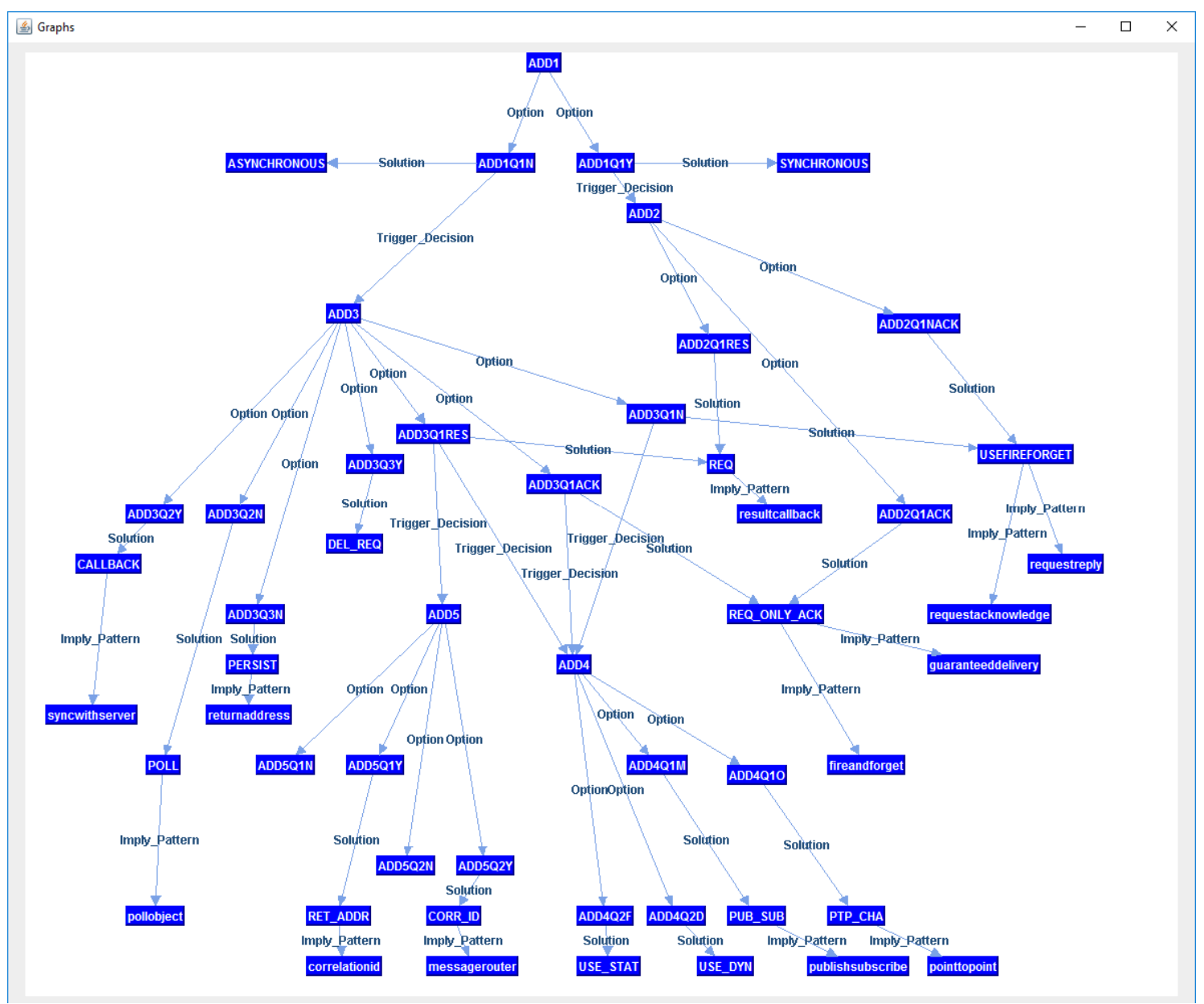

Figura 5.33. Red SBP6 sin elementos de tipo "question"

De igual manera, hemos transformado con los mismos criterios anteriormente indicados, la red SBP4 y hemos obtenido la red SBP7. 


\subsubsection{Resultados obtenidos}

Con la aplicación $A D D M-M e t r i c s$ el arquitecto puede elegir qué decisión considera origen de un cambio. A partir de ella, la aplicación creará tantos conjuntos de decisiones como nivel de profundidad de manera automática o bien, tantos niveles como el arquitecto indique. Con estos conjuntos de decisiones, el arquitecto debe analizar cuál es el número de dependencias máximo que considera necesario analizar en profundidad.

En el caso de la red adaptada SBP6 de la figura 5.33, cuando el arquitecto elige la decisión ADD1 como origen del cambio, ADDN-Metrics genera los 8 conjuntos de decisiones obtenidas mostramos en la figura 5.34 enumerados de 0 a 7 . El grupo 0 se corresponde con la decisión origen del cambio. El grupo 1 se corresponde con las decisiones cuyo nivel de dependencia es de 1, es decir, su distancia a la decisión ADD1 es de 1. Las decisiones del grupo 2 distan dos niveles de la decisión ADD1, y por lo tanto distan 1 de las decisiones del grupo 1. Este razonamiento se extiende hasta el último nivel, que en este caso es el nivel 7.

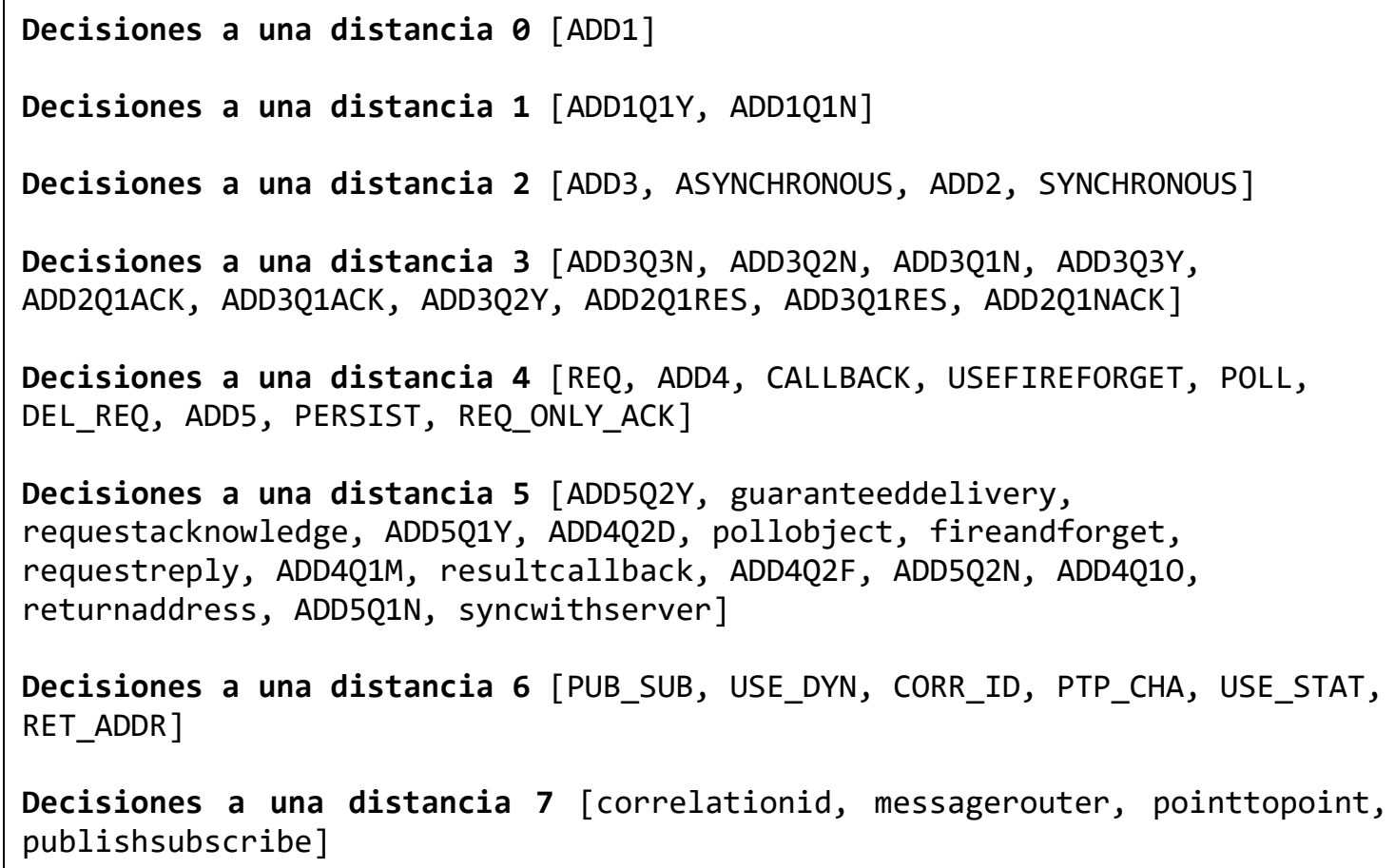

Figura 5.34. Conjuntos de decisiones impactadas en SBP6 por ADD1 
De manera opcional, $A D D N$-Metrics muestra estos resultados en el grafo de la red analizada, con el fin de ayudar al arquitecto en su análisis. En la figura 5.35, que corresponde a la red $S B P 6$, localizamos la decisión ADD1 en color rojo y el resto de decisiones impactadas en color amarillo.

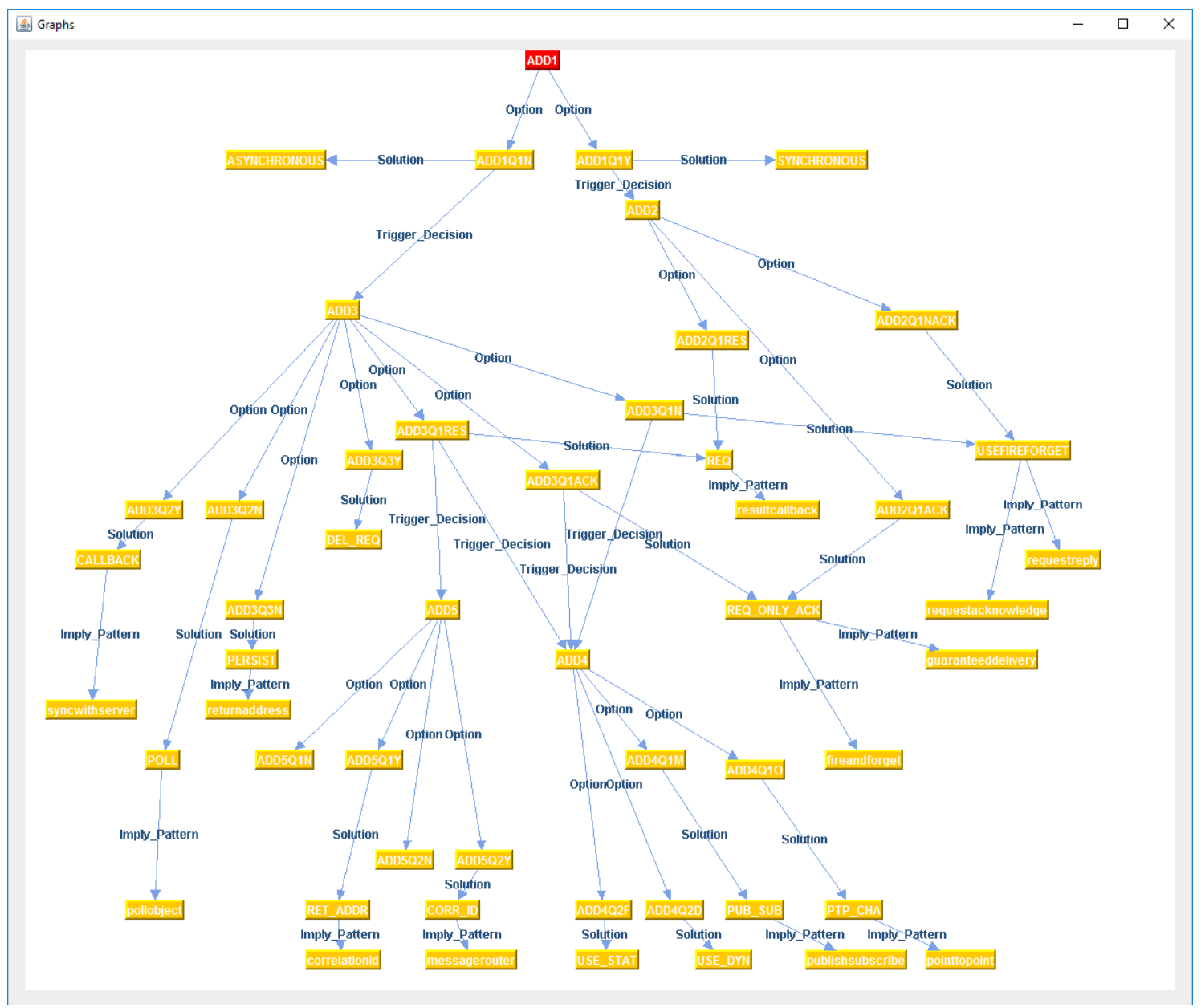

Figura 5.35. Decisiones impactadas en SBP6 por ADD1.

Siguiendo con nuestra propuesta de análisis de impacto, una vez generados los grupos de decisiones equidistantes de la decisión origen, analizamos que decisiones están más directamente afectadas por el cambio. Para ello, partiendo desde el nivel 1, analizamos cada una de las decisiones que forman parte de los grupos generados por la aplicación $A D D N-M e t r i c s$. En este caso encontramos relación entre las decisiones ADD1 y ADD3 (figura 5.36), situadas en los niveles 0 y 2 respectivamente. Este análisis lo repetimos con el resto de decisiones y no encontramos relación alguna con ADD1 a una distancia mayor de 2 (figura 5.37), por lo que consideramos que esta es la mayor distancia de impacto directo en la red. 
Como ejemplo del análisis realizado mostramos la información asociada a ADD1 y ADD3 en la figura 5.36.

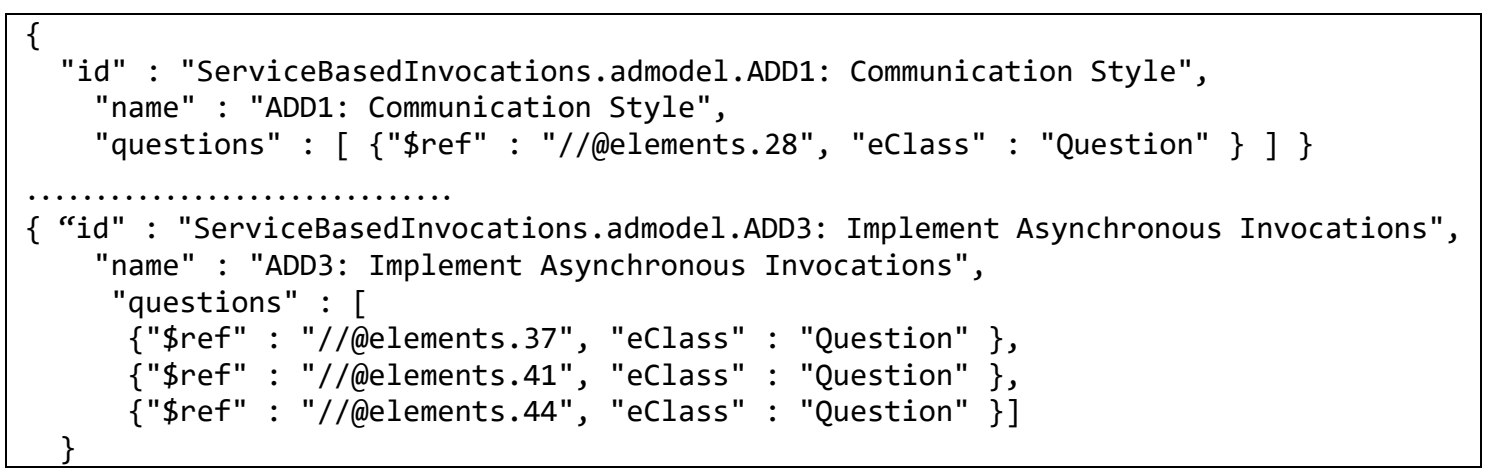

Figura 5.36. Ejemplo de relación entre decisiones

A continuación, mostramos las decisiones ADD4 y ADD5 como ejemplo de que no están relacionadas directamente con ADD1

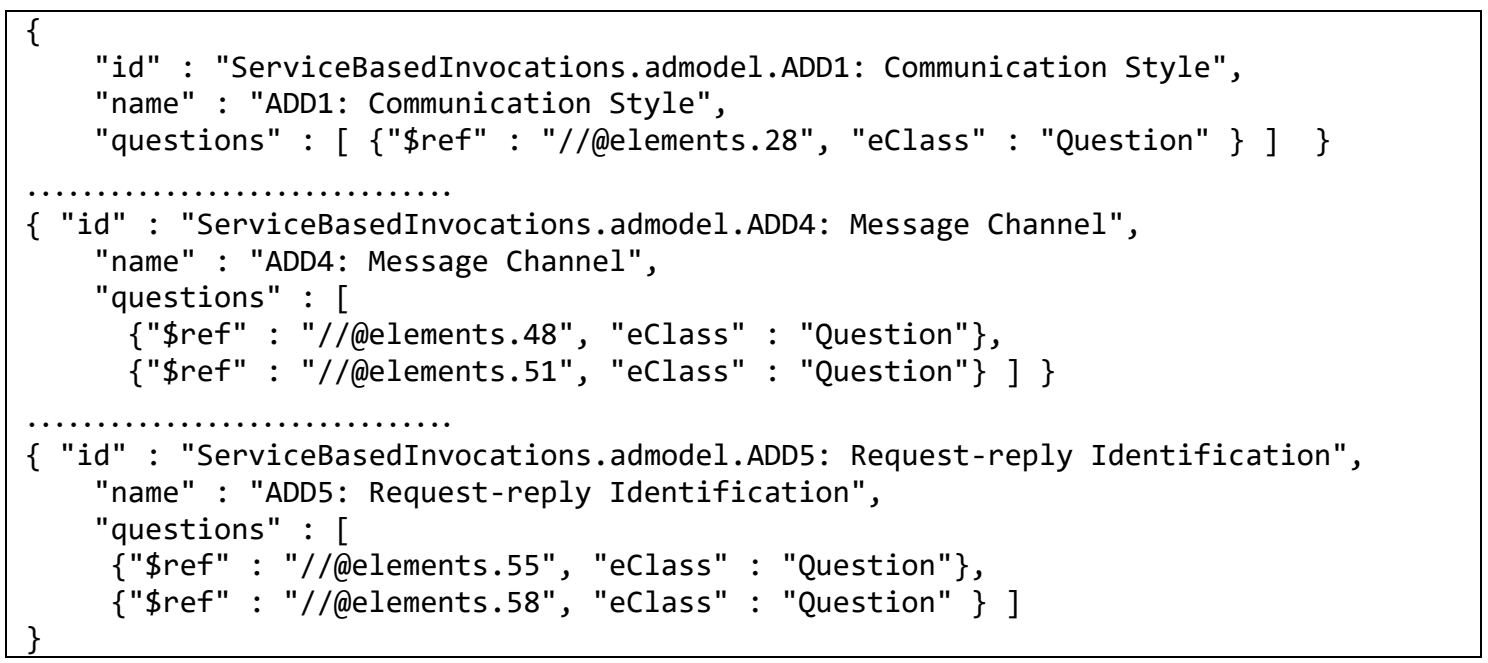

Figura 5.37. Ejemplo de no relación entre decisiones

El conjunto de decisiones afectadas por el cambio producido en ADD1 se muestra en la figura 5.38. 


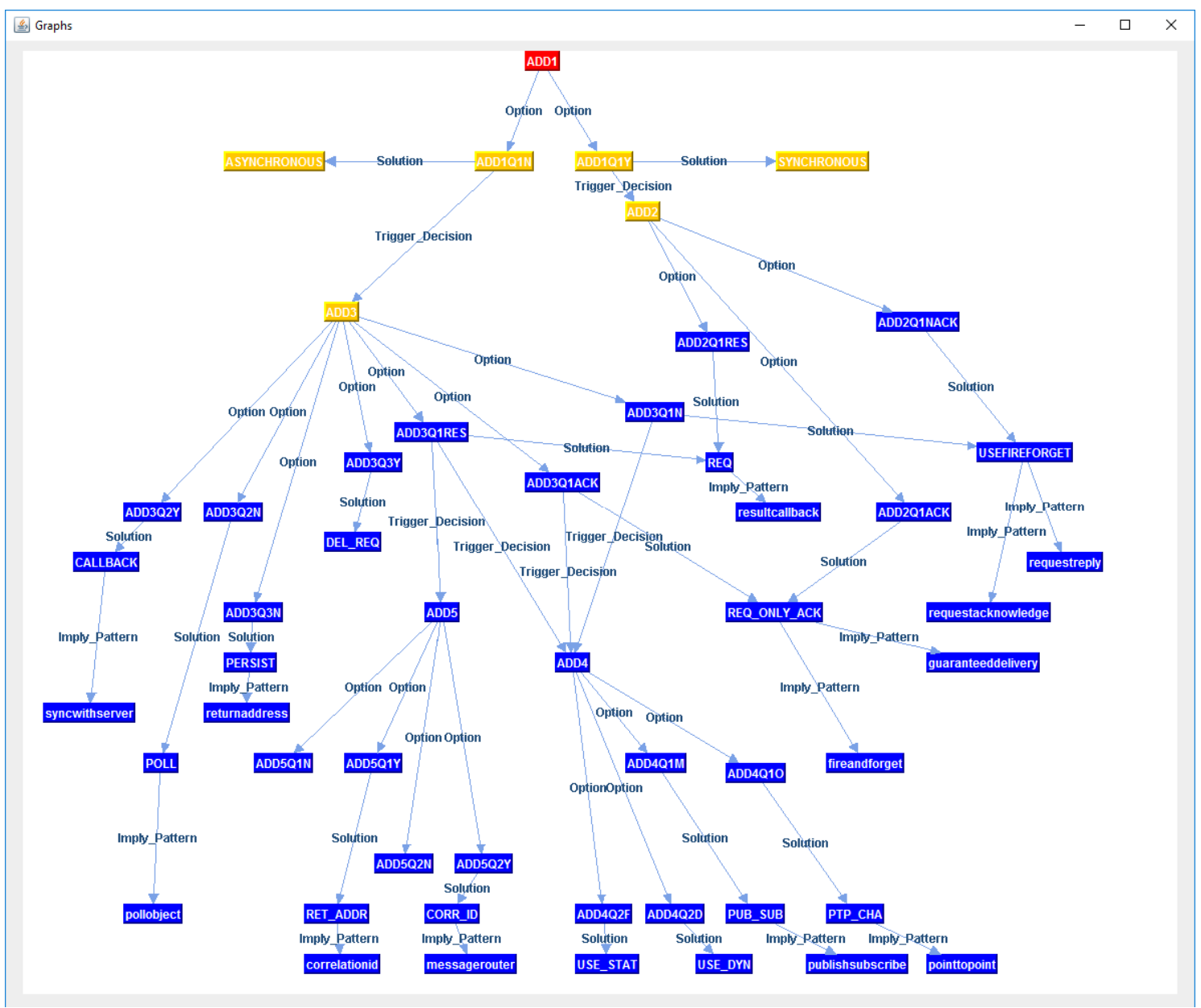

Figura 5.38. Conjuntos de decisiones impactadas en SBP6 por ADD1 en un nivel 2.

Además, se obtiene los valores de la métrica $A D D N-I$ por cada decisión impactada, tal y como mostramos en la figura 5.39, donde hemos resaltado los valores de inestabilidad más altos (más inestables) en color rojo y más bajos (más estables) en verde.

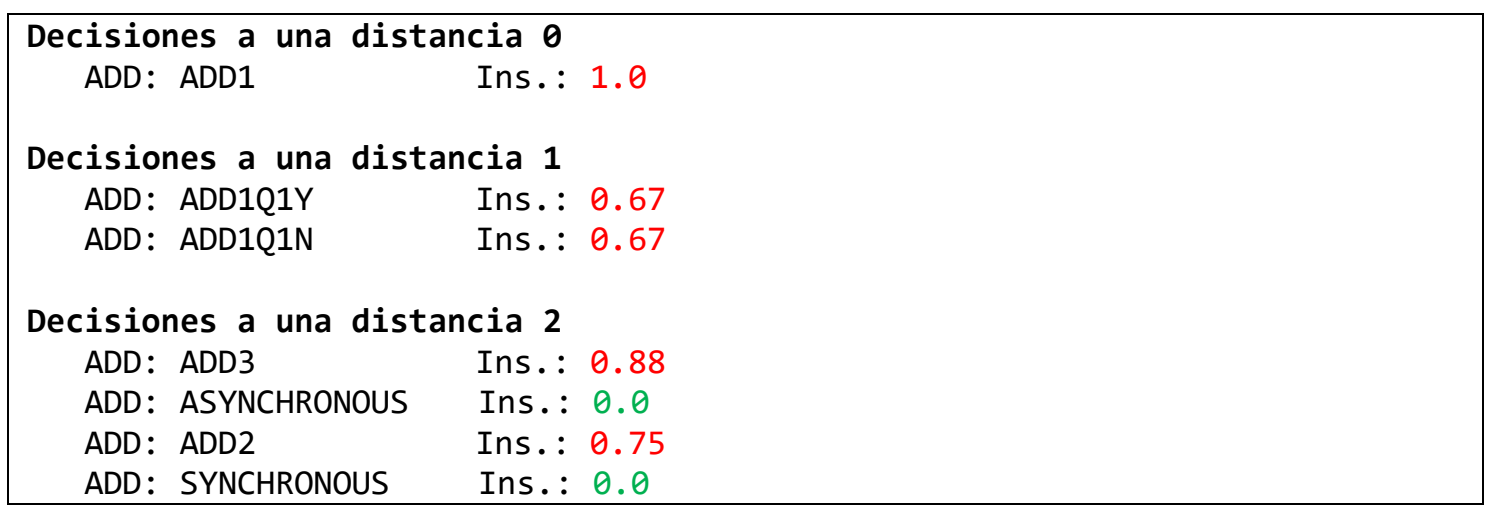

Figura 5.39. Valores de instability para las decisiones impactadas por ADD1. 
Observamos que un cambio en la decisión ADD1, con inestabilidad 1, puede afectar a ADD3 cuya inestabilidad 0.88 es muy elevada (ver criterios de inestabilidad en la sección 4.4.3, donde [ $[0 . .0 .39]$ se corresponden con decisiones estables, $[0.4 \ldots 0.6]$ con decisiones con grado de inestabilidad medio y [0.61 ... 1] con decisiones más inestables). Como observamos en la figura 6.38, ADD3 tiene 7 decisiones alternativas y cualquier cambio que afecte a ADD3 se propagará hacia sus 7 alternativas. Además, hemos resaltado las decisiones más inestables (rojo) y menos inestables (verde).

Se repite el proceso anterior, pero cambiando la decisión de impacto. Ahora, la decisión que cambia es ADD3, y nuestra aplicación genera 6 conjuntos de decisiones impactadas y que se correspondería con los conjuntos mostrados en la figura 5.40.

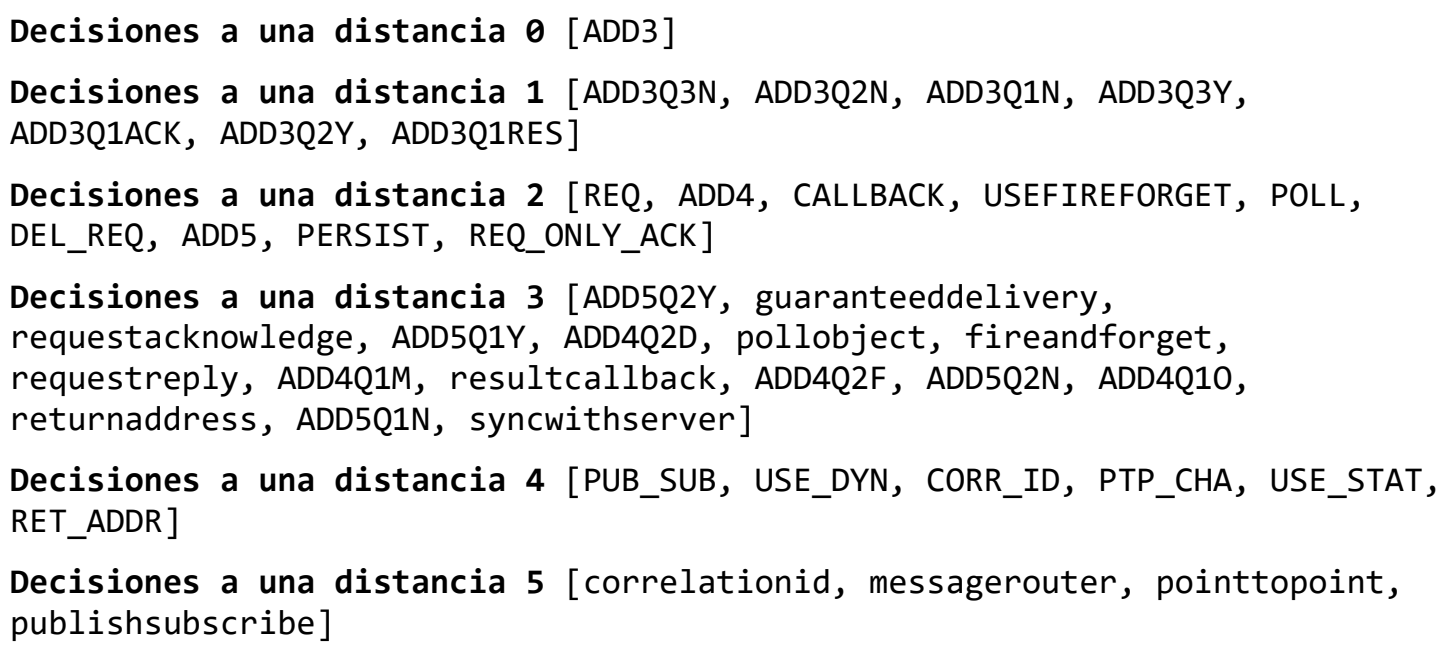

Figura 5.40. Conjuntos de decisiones impactadas en SBP6 por ADD3

Y que se corresponde con la red visualizada en la figura 5.41. 


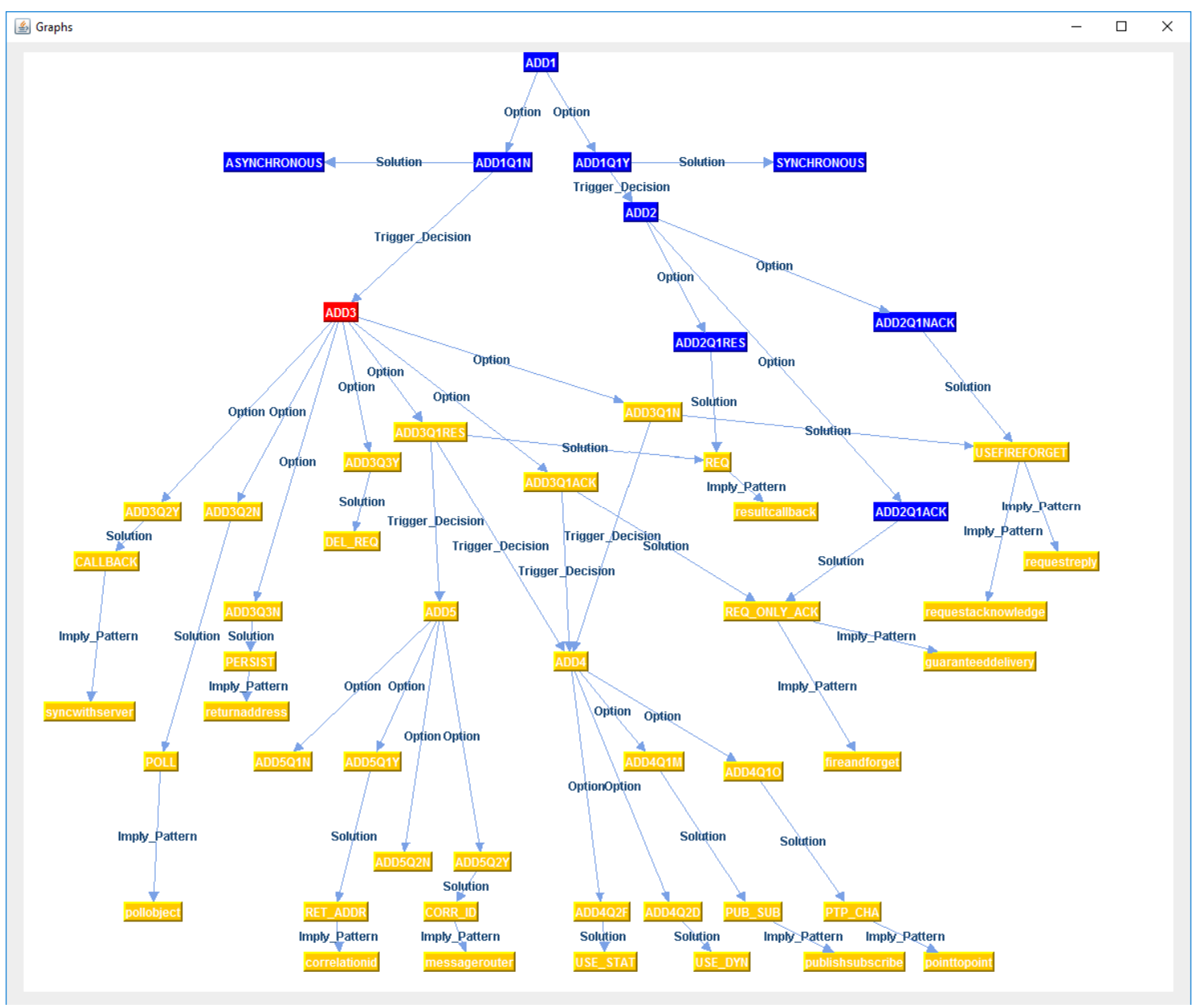

Figura 5.41. Decisiones impactadas en SBP6 por ADD3.

Se vuelve a hacer el estudio de calcular la distancia máxima de dependencia entre decisiones. En este caso, partimos de la decisión ADD3 y encontramos una dependencia conceptual con la decisión ADD5, que dista dos niveles. Este valor obtenido está en la línea del estudio anterior para esta misma red. De nuevo, obtenemos las decisiones impactadas a una distancia de 2, y los valores de instability calculados para cada decisión, tal y como se muestra en la figura 5.42, donde hemos resaltado los valores de inestabilidad más altos (más inestables) en color rojo y más bajos (más estables) en verde. 


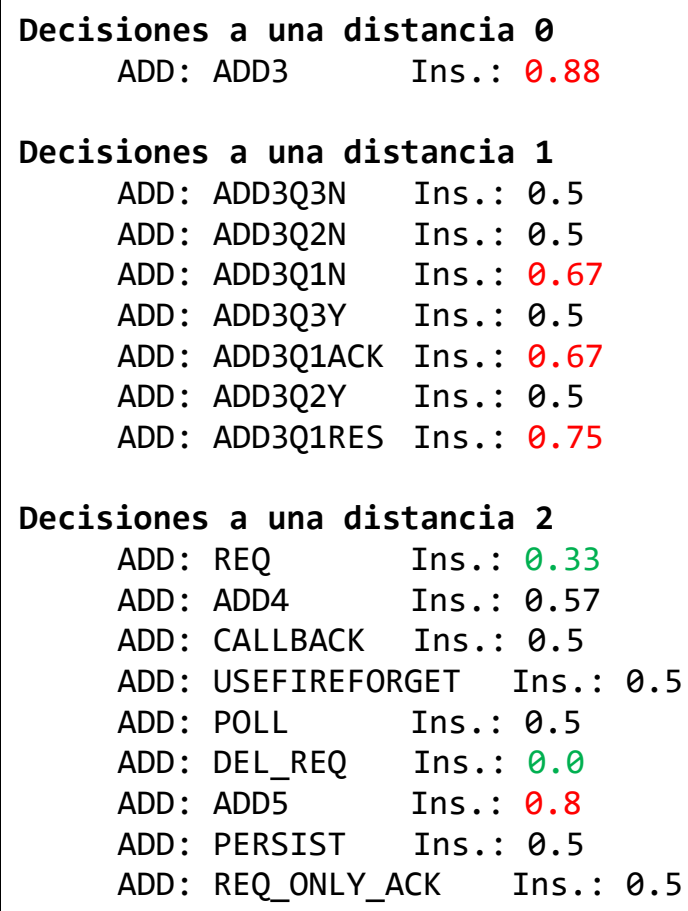

Figura 5.42. Valores de instability para las decisiones impactadas por ADD3

Con los valores de inestabilidad obtenidos, y con los criterios de inestabilidad indicados en la sección 4.4.3, observamos que las decisiones dependientes de ADD3 no son altamente inestables, salvo la decisión ADD3Q1RES, que tiene 0.75 y que a su vez está conectada con la decisión ADD5, que tienen un valor de 0.8 , por lo que se deduce que un cambio en ADD3 se propagará más fácilmente hacia ADD5 (distante dos niveles de ADD3) a través de ADD3Q1RES, por ser estas tres decisiones altamente inestables.

Y este conjunto de decisiones impactadas por ADD3 se visualiza en la figura 5.43 


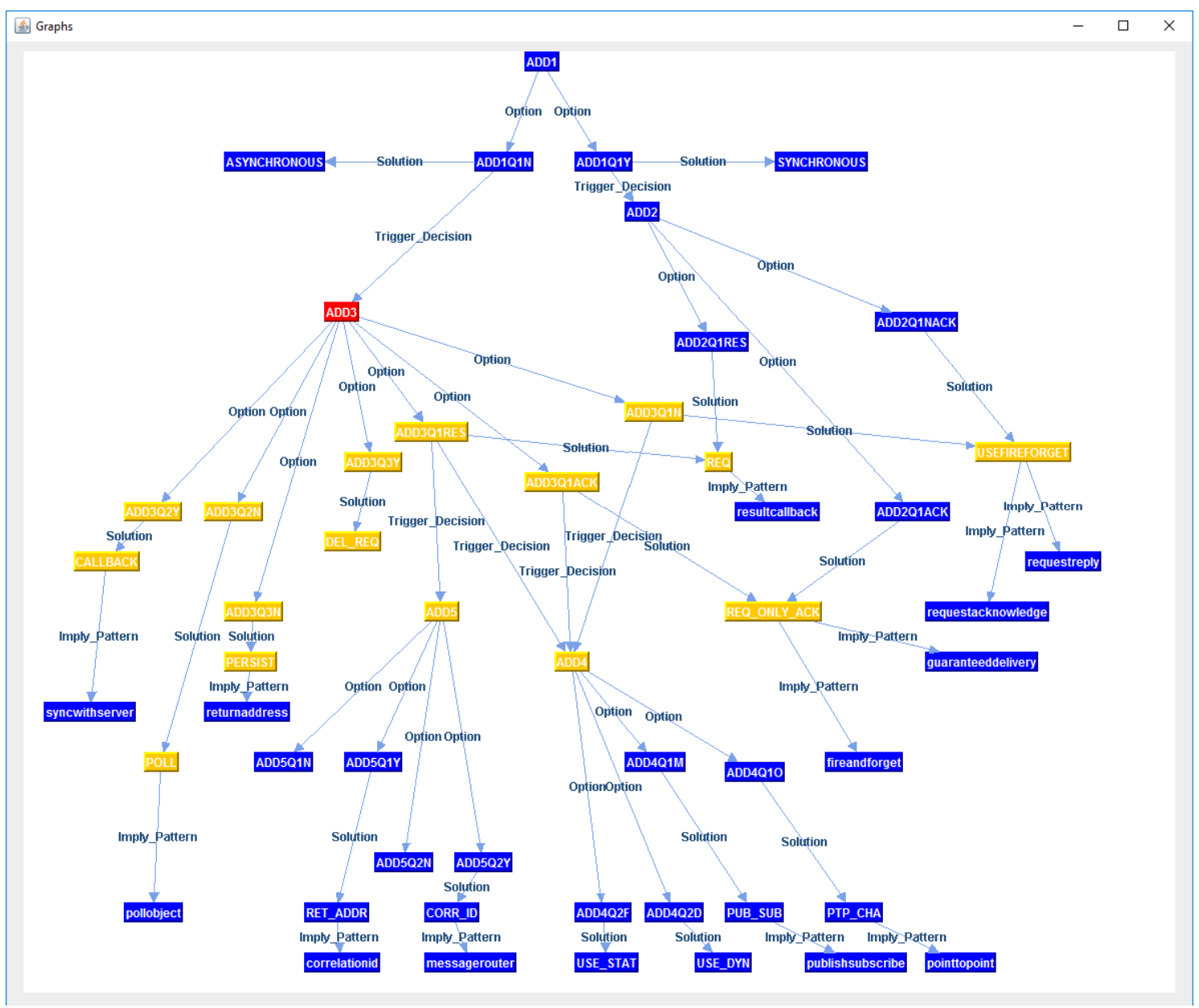

Figura 5.43. Conjuntos de decisiones impactadas en SBP6 por ADD3 en un nivel 2.

Por último, en esta red se analiza que decisiones se verán afectadas si se produce un cambio en una alternativa, por ejemplo ADD3Q1RES. A continuación, mostramos los resultados obtenidos en la figura 5.44.

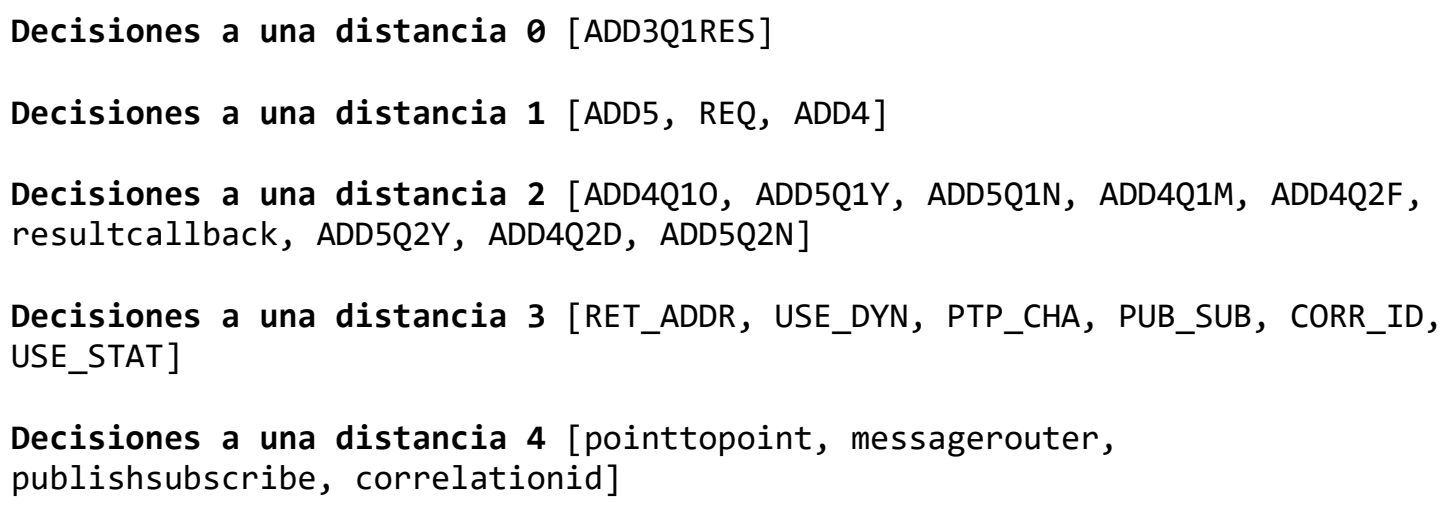

Figura 5.44. Conjuntos de decisiones impactadas en SBP6 por ADD3Q1RES

Y de manera opcional se visualiza el conjunto de decisiones impactadas por ADD3Q1RES, tal y como se visualiza en la figura 5.45. 


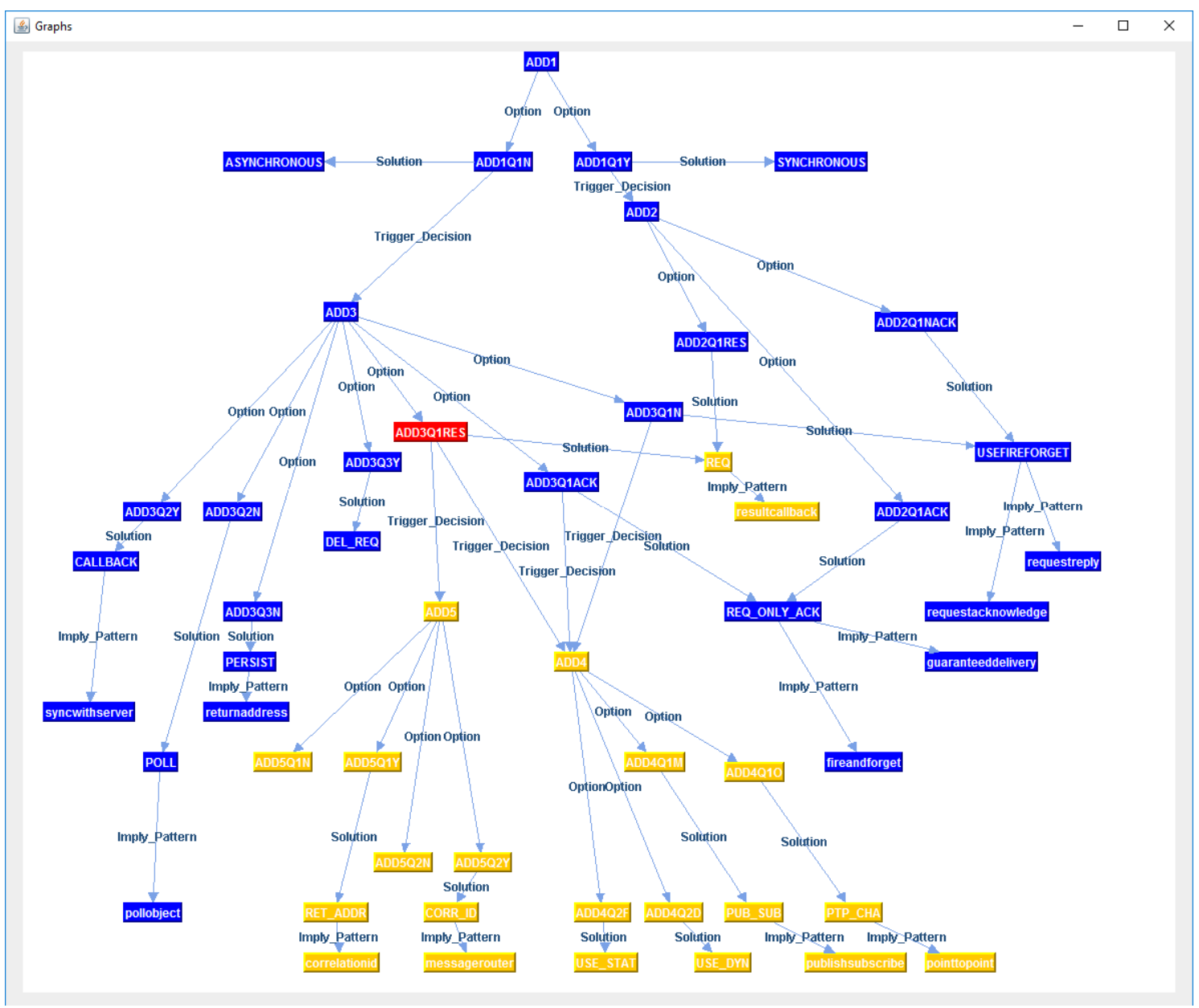

Figura 5.45. Decisiones impactadas en SBP6 por ADD3.

Se vuelve a realizar el estudio para determinar la distancia máxima de impacto, y en este caso se encuentra que es 1 nivel. Generalizando para toda la red, y en base a los tres estudios realizados, llegamos a la conclusión que, para esta red, la distancia a calcular es de 2, por lo que el grafo de decisiones impactadas se corresponde con la figura 5.46. 


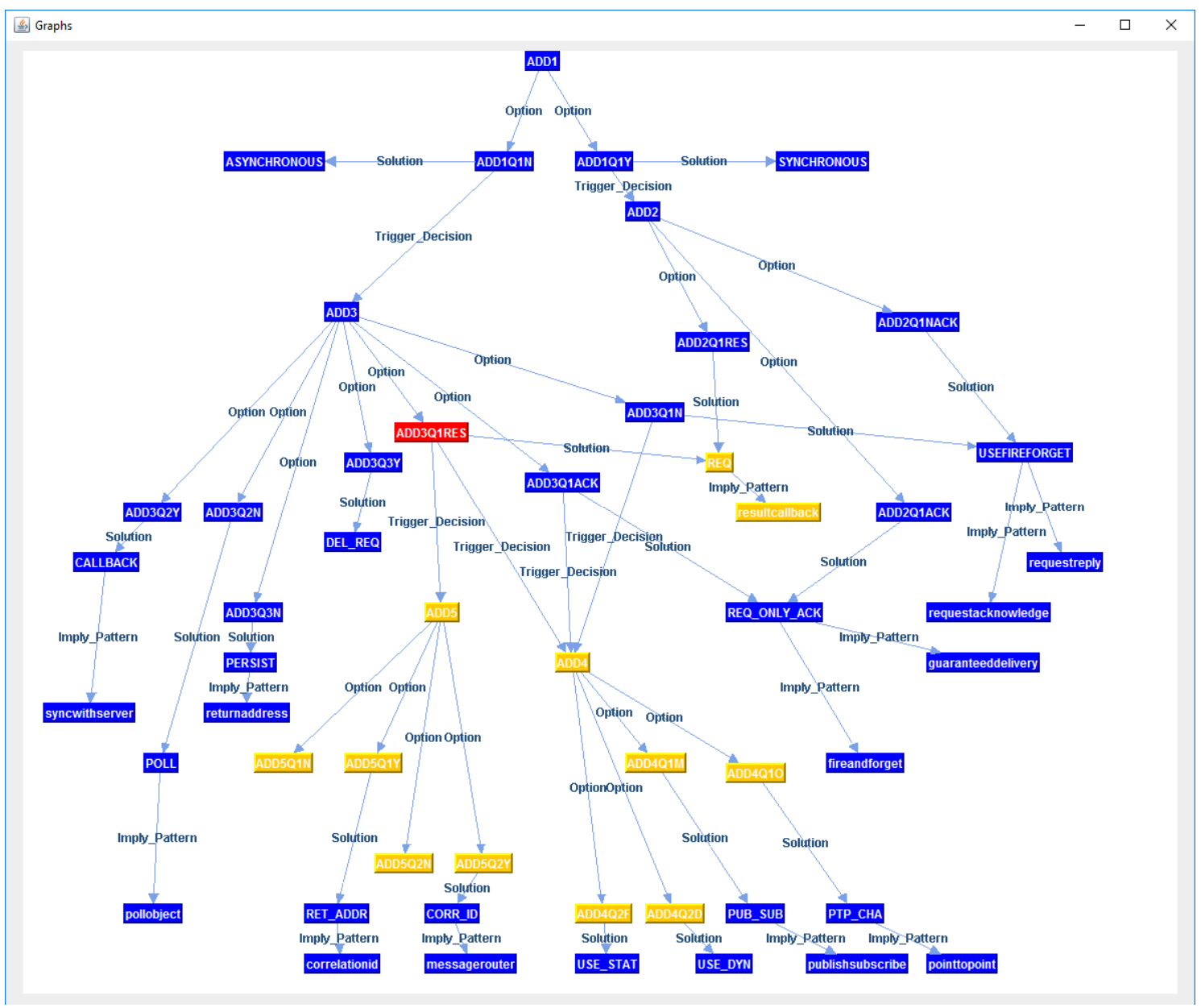

Figura 5.46. Conjuntos de decisiones impactadas en SBP6 por ADD3 en un nivel 2.

Los valores de instability obtenidos para este conjunto de decisiones impactadas se muestra en la figura 5.47, donde hemos resaltado los valores de inestabilidad más altos (más inestables) en color rojo y más bajos (más estables) en verde.

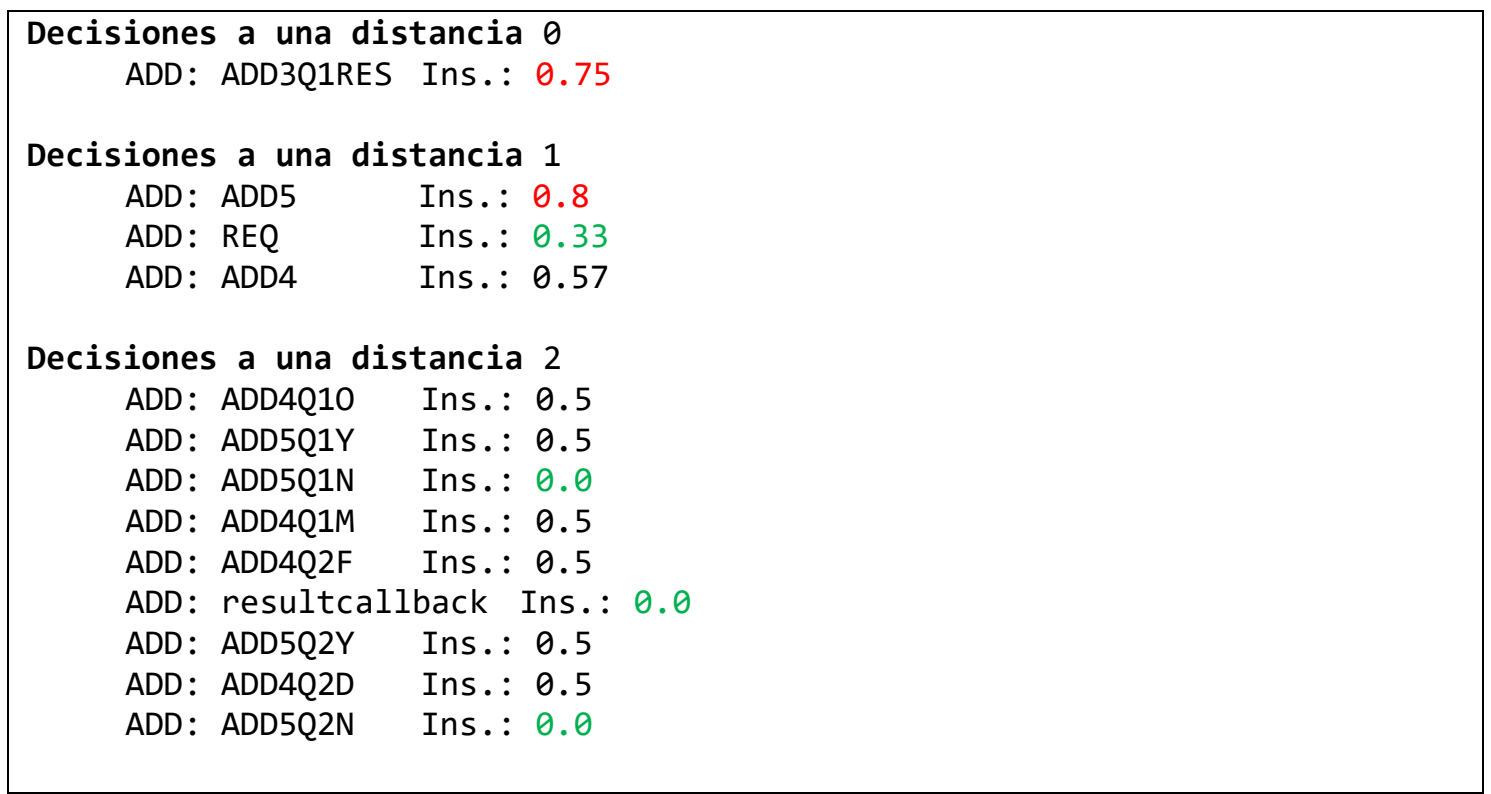

Figura 5.47. Valores de instability para las decisiones impactadas por ADD3 
Aplicando los criterios de inestabilidad propuestos, un cambio en ADD3Q1RES afecta en mayor medida a ADD5, ya que tiene un valor de inestabilidad de $0.8 \mathrm{y}$ el resto de decisiones impactadas son medianamente estables.

Hemos reproducido este experimento en la red SBP7. En este caso, únicamente hemos generado los grupos de decisiones impactadas, y obtenemos los datos representados en la figura 5.48 .

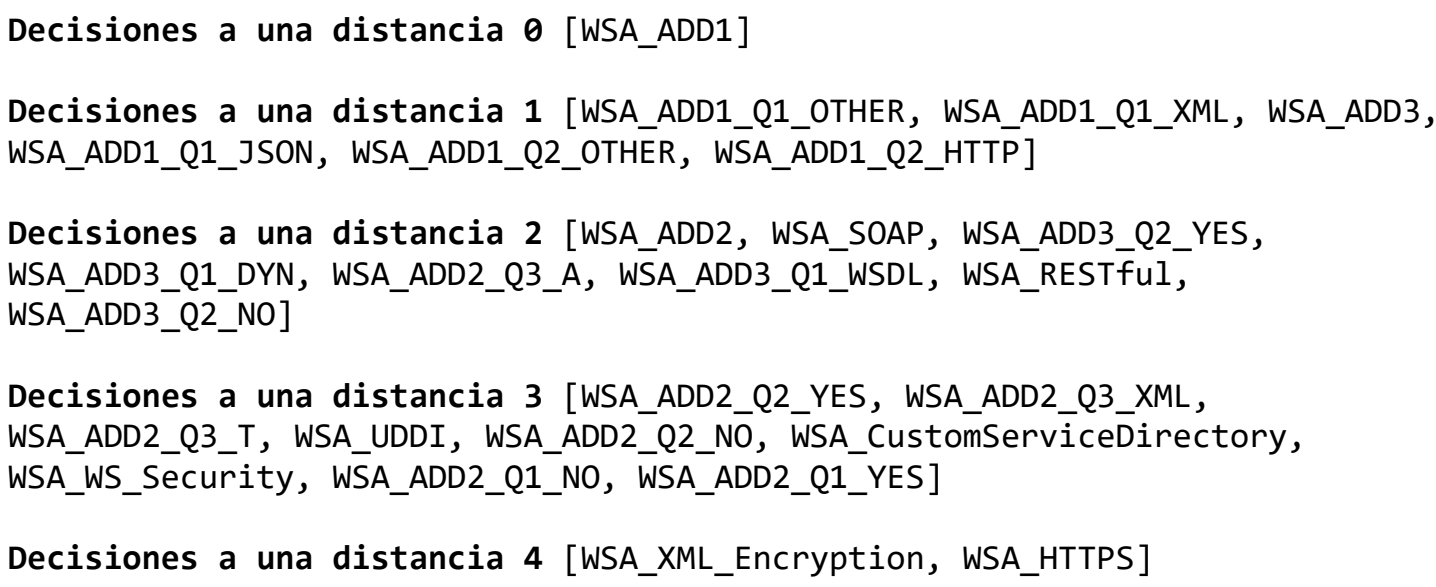

Figura 5.48. Conjuntos de decisiones impactadas en SBP7 por WSA_ADD1.

A continuación, realizamos el estudio de las descripciones de las decisiones para determinar la distancia máxima, y en esta red la distancia es de 1. En esta red, las decisiones impactadas se visualizan en la figura 5.49, junto a los valores de instability calculados.

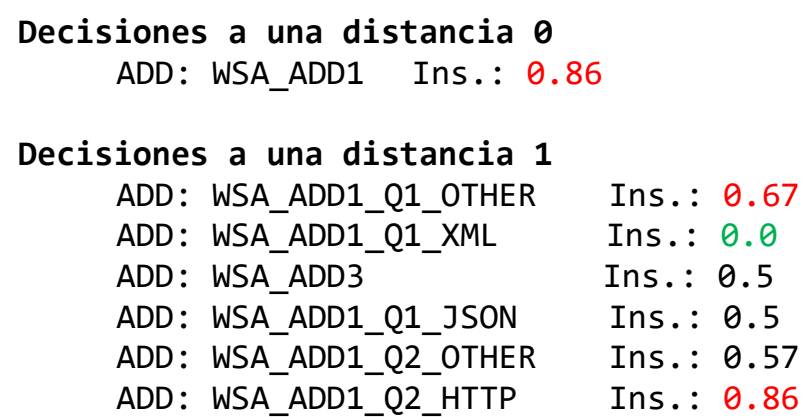

Figura 5.49. Valores de instability para las decisiones impactadas por WSA_ADD1. 


\subsubsection{Análisis de los resultados}

De los resultados obtenidos al comparar las decisiones de diseño proporcionadas por el algoritmo y la decisión de partida, debido a la pobre descripción de las decisiones, solo podemos concluir que el número de niveles a explorar es de 2. De este conjunto pertenecientes hasta nivel 2, los valores más inestables corresponden a decisiones entre $[0,61 \ldots 1]$ y los más estables están en el intervalo [0...0,39]. Del resto de las decisiones no podemos arrojar resultados concluyentes sobre su estado de estabilidad. En concreto, del experimento realizado y eliminando los casos extremos con valores 0 o 1 , en general obtenemos un mayor número de decisiones inestables que estables, aunque muchas de ellas obtienen un valor de estabilidad medio. Por ello, podríamos asesorar al arquitecto software en centrarse en el impacto de aquellas decisiones con un valor igual o superior a 0,61 .

En base a la experimentación realizada, podemos concluir que, en redes de decisiones unidireccionales, es recomendable analizar decisiones de diseño distantes como máximo de dos niveles con la decisión origen del cambio. Además del análisis realizado en la documentación disponible para este trabajo, la combinación con los datos de instability. Además, hay que tener en cuenta el criterio y experiencia del arquitecto al realizar este estudio de propagación de impacto

\subsubsection{Limitaciones de la experimentación}

Validez interna: En el experimento no utilizamos grupos de test ni de control ya que no estábamos comparando aspectos diferentes en la estimación de coste en la captura de decisiones de diseño. Sin embargo, una de las limitaciones para la validad de los resultados obtenidos por los alumnos es en la estimación de tiempo o esfuerzo en la captura y en reflexionar en las decisiones, ya que no medimos el tiempo exacto del esfuerzo debido a que parte de él se realizó fuera del laboratorio, por lo que es posible que algunos grupos hayan manifestado tiempos muy pequeños o muy grandes. Otras de las limitaciones que hemos detectado que no hemos realizado grabaciones de voz o de video de los sujetos acerca del uso del modelo Mind1-Mind2, por lo que resulta difícil contrastar la fiabilidad de las reflexiones. 
Capítulo 5. Experimentación

Validez externa: Acerca de los resultados obtenidos no podemos generalizar las conclusiones ya que este es el primer experimento de este tipo que conocemos y se necesitaría replicar dicho experimento y también llevarlo a cabo en un contexto industrial. Otra amenaza a la validez del experimento es que la información sobre las decisiones en las redes "Services-based Platform" (SBP) y sus variantes es poco detallada debido a que estas redes las ha proporcionado un colaborador externo. 


\section{Capítulo 6. Conclusiones}

Realizada la experimentación, en este capítulo exponemos las conclusiones extraídas del trabajo de investigación realizado, así como las propuestas de mejora, futuras líneas de investigación y los resultados de nuestra actividad investigadora ligada a este trabajo.

\subsection{Conclusiones Generales}

A lo largo de este trabajo hemos destacado una falta de métricas para estimar la sostenibilidad en las arquitecturas y en las decisiones de diseño. De la solución aportada podemos arrojar las siguientes conclusiones:

- Nuestro meta-modelo flexible y configurable sugiere elementos para medir la sostenibilidad en arquitecturas software.

- La definición de un conjunto de criterios que nos relacionan métricas con medidas de calidad que individualmente o combinadas permiten estimar aspectos de sostenibilidad en el conocimiento arquitectónicos.

- Proponer un conjunto de métricas y recomendaciones para medir la sostenibilidad de las decisiones de diseño atendiendo a los criterios aportados en la solución.

\subsection{Conclusiones de la Experimentación}

Las conclusiones más visibles que podemos extraer sobre la experimentación realizada son las siguientes:

- Limitar la cantidad del conocimiento a capturar en base a un número de atributos configurable y al número de alternativas favorece a su sostenibilidad.

- La topología de las diferentes redes de decisiones influye en la medida de complejidad, pero es impredecible por parte del arquitecto software conocer cuál va a ser la forma final de una red de decisiones. Sin embargo, de las observaciones realizadas podemos asesorar que topologías son más complejas. 
- La combinación de medidas de ripple effect y de instability da una mayor precisión al arquitecto para evaluar las decisiones impactadas por un cambio y prestar mayor atención a aquellas con un mayor grado de inestabilidad. Además, nos permite identificar las decisiones que tienen a perdurar más.

\subsection{Futuras Líneas}

Una vez completado nuestro trabajo, planteamos las futuras líneas de investigación:

- Sugerir una mayor combinación de métricas para obtener resultados más precisos.

- Analizar la estabilidad de la arquitectura software en base a su evolución contando con diferentes versiones de la arquitectura.

- Estudiar el efecto del ripple effect cuando se añaden y se eliminan decisiones.

- Validar nuestras propuestas de métricas con redes arquitectónicas más complejas.

- Integrar nuestras métricas con herramientas que miden aspectos de deuda técnica.

\subsection{Producción Científica}

En esta sección señalamos nuestra contribución científica generada a partir de los trabajos de investigación desarrollados en esta Tesis Doctoral. En el Anexo VIII incluimos cada uno de nuestros resultados, que a continuación los cito:

\section{Revistas/Magazines}

Toward Architecture Knowledge Sustainability. Extending System Longevity.

R. Capilla, E. Y. Nakagawa, U. Zdun, and C. Carrillo

In IEEE Software, vol. 34, no. 2, Page(s): 108-111, Mar.-Apr. 2017.

\section{Capítulos de libro}

Capítulo 13: An Overview on Quality Evaluation of Self-Adaptive Systems.

C. Raibulet, F. Arcelli Fontana, R. Capilla y C. Carrillo.

Título libro: Managing Trade-Offs in Adaptable Software Architectures,

Editoral: Elsevier, Editores: I. Mistrik, N. Ali, R. Kazman, J. Grundy, B. Schmerl, Page(s):325-352. Enero 2017. 


\section{Conferencias internacionales}

Relating Architectural Decay and Sustainability of Software Systems D. M. Le, C. Carrillo, R. Capilla and N. Medvidovic.

2016 13th Working IEEE/IFIP Conference on Software Architecture (WICSA), Venice, 2016, Page(s): 178-181. 2016.

Guidelines and Metrics for Configurable and Sustainable Architectural Knowledge Modelling.

C. Carrillo, R. Capilla, O. Zimmermann and U. Zdun.

In Proceedings of the 2015 European Conference on Software Architecture Workshops (ECSAW '15). ACM, New York, NY, USA, Article 63, 5 pages. 2015.

Effort Estimation in Capturing Architectural Knowledge.

R. Capilla, F. Nava and C. Carrillo.

ASE '08 Proceedings of the 2008 23rd IEEE/ACM International Conference on Automated Software Engineering. Page(s): 208-217 IEEE Computer Society. 2008.

ADDSS: Architecture Design Decision Support System Tool.

R. Capilla, F. Nava, J. Montes and C. Carrillo.

2008 23rd IEEE/ACM International Conference on Automated Software

Engineering, L'Aquila, 2008, Page(s): 487-488. 2008.

\section{Prepación para revista}

A Systematic Literature Review on Quality Attributes Use in Architectural Design Decision Methods

I. Lytra, C. Carrillo, R. Capilla and U. Zdun

Information and Software Technology Journal

A Survey on Sustainability Dimensions: Research and Practice from a Software Architecture Point of View,

C. C. Venters, R. Capilla, S. Betz, B. Penzenstadler, T. Crick, S. Crouch, E. Y. Nakagawa, C. Becker and C. Carrillo,

Journal and Systems Software.

\section{Enviados para congresos internacionales}

Towards Measuring the Complexity of Architectural Decision Networks C. Carrillo and R. Capilla.

11th European Conference on Software Architecture (ECSA 2017).

Toward Sustainability Metrics for Autonomous Systems

R. Capilla, M. Staron, A. Martini, D. Le, C. Carrillo and N. Medvidovic.

11th European Conference on Software Architecture (ECSA 2017). 


\section{Capítulo 7. Referencias}

[AKER 06] A. Akerman and J. Tyree.

Using Ontology to Support Development of Software Architectures.

IBM Systems Journal. Page(s): 613 -825. Vol. 45, nº 3; Jul-Sep 2006.

[AMEL 13] D. Ameller, C. Ayala, J. Cabot and X. Franch

Non-Functional Requirements in Architectural Decision Making.

Software, IEEE, 30(2). Page(s): 61-67. 2013

[AMPA 15] A. Ampatzoglou, A. Chatzigeorgiou, S. Charalampidou and P. Avgeriou, The Effect of GoF Design Patterns on Stability: A Case Study. In IEEE Transactions on Software Engineering, vol. 41, no. 8. Page(s): 781-802. 2015.

[AVGE 13] P. Avgeriou, M. Stal and R. Hilliard. Architecture Sustainability.

IEEE Software November/December (4). Page(s): 40-45. 2013.

[BABA 05] M.A. Babar, X. Wang and I. Gorton,

PAKME: A Tool for Capturing and Using Architecture Design

Knowledge.

2005 Pakistan Section Multitopic Conference. Page(s): 1-6. 2005

[BABA 07] M.A. Babar and I. Gorton.

A Tool for Managing Software Architecture Knowledge.

Proceedings of the 2nd Workshop on Sharing and Reusing Architectural Knowledge, Page(s): 11, ISBN: 0-7695-2951-8, 2007.

[BABA 08] M.A. Babar, A. Northway, I. Gorton, P. Heuer and T. Nguyen. Introducing Tool Support for Knowledge Management: An Experience Report

15th Annual IEEE International Conference and Workshop on the Engineering of Computer Based Systems. Page(s): 105-113. 2008.

[BARA 16] M. Al-Barak and R. Bahsoon.

Database Design Debts through Examining Schema Evolution.

IEEE 8th International Workshop on Managing Technical Debt (MTD), Raleigh, NC, 2016, pp. 17-23. 2016

[BASS 98] L. Bass, P. Clements and R. Kazman.

Software Architecture in Practice.

Addison-Wesley, 1998.

[BASS 13] L. Bass, P. Clements and R. Kazman.

Software Architecture in Practice, 3rd Edition.

Addison-Wesley, ISBN: 0-321-81573-4, 2013. 
[BHAT 16] M. Bhat, K. Shumaiev, A. Biesdorf, U. Hohenstein, M. Hassel, and F. Matthes.

Meta-model based framework for architectural knowledge management. In Proccedings of the 10th European Conference on Software Architecture Workshops (ECSAW '16). ACM, Article 12, 7 pages. 2016.

[BECK 14] C. Becker, R. Chitchyan, L. Duboc, S. Easterbrook, M. Mahaux, B. Penzenstadler, G. Rodriguez-Navas, C. Salinesi, N. Seyff, C. Venters, C. Calero, S. A. Kocak, and S. Betz, The Karlskrona Manifesto for Sustainability Design. 2014.

[BECK 15] C. Becker, R. Chitchyan, L. Duboc, S. Easterbrook, B. Penzenstadler, N. Seyff and C. Venters. Sustainability Design and Software: The Karlskrona Manifesto. IEEE/ACM 37th IEEE International Conference on Software Engineering (ICSE), 2015 (Volume: 2), Page(s): 467-476. 2015.

[BETZ 15] S. Betz, C. Becker, R. Chitchyan, L. Duboc, S. Easterbrook, B. Penzenstadler, N. Seyff and C. Venters.

Sustainability Debt: A Metaphor to Support Sustainability Design Decisions.

Proceedings of the Fourth International Workshop on Requirements Engineering for Sustainable Systems, RE4SuSy 2015. Page(s): 55-63. 2015.

[BODE 10] S. Bode and M. Riebisch. Impact Evaluation For Quality-Oriented Architectural Decisions Regarding Evolvability. In European Conference on Software Architecture. Page(s): 182-197, Springer Berlin Heidelberg. 2010

[BOER 07] R. C. de Boer, R. Farenhorst, P. Lago, H. van Vliet, V. Clerc and A. Jansen.

Architectural Knowledge: Getting to the Core.

Eds.: Overhage et al. QoSA 2007, LNCS 4880. Page(s): 197-214, Springer-Verlag Berlin Heidelberg 2007.

[BOER 17] R. C. de Boer ArchiMedes - Publication and Integration of Architectural Knowledge. IEEE International Conference On Software Architecture (ICSA 2017), 2017.

[BOSC 04] J. Bosch.

Software Architecture: The Next Step.

Proceedings of the 1st European Workshop on Software Architecture (EWSA 2004). Page(s): 194-199, Springer-Verlag, LNCS 3047, 2004.

[BRIA 96] L. Briand, S. Morasca, and V. Basili.

Property-Based Software Engineering Measurement. IEEE Trans. Softw. Eng. 22, 1, Page(s): 68-86. 1996. 
[BUSC 96] F. Buschmann, R. Meunier, H. Rohnert, P. Sommerlad and M. Stal. Pattern-Oriented Software Architecture. Ed. John Wiley \& Sons, ISBN: 978-0-471-95869-7, 1996.

[CABO 09] J. Cabot, S. Easterbrook, J. Horkoff, J. Mazón, L. Lessard and S. Liaskos. Integrating Sustainability in Decision-Making Processes: A Modelling Strategy. 31st International Conference on Software Engineering -Companion. ICSE-Companion 2009. ISBN 978-1-4244-3495-4. 2009

[CALE 13] C. Calero, M.A. Moraga and M. F. Bertoa. Towards A Software Product Sustainability Model. WSSSPE'1: First Workshop on sustainable software for science: practice and experiences, SC'13, 17 November 2013, 2013.

[CAPI 06] R. Capilla, F. Nava, S. Pérez and J.C. Dueñas.

A Web-based Tool for Managing Architectural Design Decisions. Proceedings of the 1st Workshop on Sharing and Reusing Architectural Knowledge, ACM Digital Library, Software Engineering Notes 31 (5) Article No. 4, ISSN: 0163-5948, 2006.

[CAPI 07A] R. Capilla, F. Nava and J.C. Dueñas.

Modeling and Documenting the Evolution of Architectural Design Decisions.

Proceedings of the 2nd Workshop on Sharing and Reusing Architectural Knowledge, ICSE Workshops, ISBN: 0-7695-2951-8, 2007.

[CAPI 07B] R. Capilla, F. Nava and A. Tang. Attributes for Characterizing the Evolution of Architectural Design Decisions.

Third International IEEE Workshop on Software Evolvability. Page(s): 15-22. ISBN: 978-0-7695-3002-4. 2007.

[CAPI 08] R. Capilla, F. Nava and C. Carrillo. Effort Estimation in Capturing Architectural Knowledge. ASE '08 Proceedings of the 2008 23rd IEEE/ACM International Conference on Automated Software Engineering. Page(s): 208-217 IEEE Computer Society. 2008.

[CAPI 08B] R. Capilla, F. Nava, J. Montes and C. Carrillo. ADDSS: Architecture Design Decision Support System Tool. 2008 23rd IEEE/ACM International Conference on Automated Software Engineering, L'Aquila, 2008, pp. 487-488. 2008 
[CAPI 11] R. Capilla, O. Zimmermann, U. Zdun, P. Avgeriou and J.M. Küster. An Enhanced Architectural Knowledge Metamodel Linking Architectural Design Decisions to other Artefacts in the Software Engineering Lifecycle.

ECSA 2011, LNCS 6903. Page(s): 303-318, Springer-Verlag Berlin Heidelberg 2011.

[CAPI 16] R. Capilla, A. Jansen, A. Tang, P. Avgeriou and M. Ali Babar, 10 Years of Software Architecture Knowledge Management: Practice and Future.

Journal of Systems and Software, Volume 116 Issue C, June 2016 Page(s): 191-205. Elsevier Inc. 2016

[CAPI 17] R. Capilla, E. Y. Nakagawa, U. Zdun, and C. Carrillo Toward Architecture Knowledge Sustainability. Extending System Longevity. In IEEE Software, vol. 34, no. 2, Page(s): 108-111, Mar.-Apr. 2017.

[CARR 15] C. Carrillo, R. Capilla, O. Zimmermann and U. Zdun Guidelines and Metrics for Configurable and Sustainable Architectural Knowledge Modelling. In Proceedings of the 2015 European Conference on Software Architecture Workshops (ECSAW '15). ACM, New York, NY, USA, Article 63, 5 pages. 2015.

[CHID 94] S. R. Chidamber and C. F. Kemerer, A metrics suite for object oriented design. In IEEE Transactions on Software Engineering, vol. 20, no. 6, Page(s): 476-493, 1994.

[CLEL 03] J. Cleland-Huang, C.K. Chang and M. Christensen. Event-Based Traceability for Managing Evolutionary Change. IEEE Transactions on Software Engineering 29 (9). Page(s): 796-810. 2003.

[CLEM 02] P. Clements, F. Bachman, L. Bass, D. Garlan, J. Ivers, R. Little, R. Nord and J. Stafford.

A Practical Method for Documenting Software Architectures. 2002.

[CLEM 03] P. Clements, F. Bachman, L. Bass, D. Garlan, J. Ivers, R. Little, R. Nord and J. Stafford.

Documenting Software Architectures. Views and Beyond. Addison-Wesley, ISBN: 0-201-70372-6, 2003.

[CONS 15] E. Constantinou and I. Stamelos.

Architectural Stability and Evolution Measurement for Software Reuse. In Proceedings of the 30th Annual ACM Symposium on Applied Computing SAC'15. Page(s): 1580-1585. ACM DL. 2015. 
[CUES 13] C. E. Cuesta, E. Navarro, D. E. Perry and C. Roda.

Evolution Styles: Using Architectural Knowledge as an Evolution Driver. Journal of Software: Evolution and Process, 25(9), 957-980. 2013

[CUNN 92] W. Cunningham.

The WyCash Portfolio Management System.

Proc. OOPSLA, ACM, 1992; http://c2.com/doc/oopsla92.html.

[DAND 15] Z. G. Dand and H. Vasishtha.

Analysis and Evaluation of Quality Metrics in Software Engineering. International Journal of Advanced Research in Computer and Communication Engineering, IJARCCE. Vol. 4, no 4. Page(s): 235-240. 2015.

[DHAY 12] C. Dhaya and G. Zayaraz.

Fuzzy Based Quantitative Evaluation of Architectures using Architectural Knowledge. International Journal of Advanced Science and Technology, Vol 49. 2012.

[DIAZ 11] J. Díaz, J. Perez, J. Garbajosa and A. L. Wolf. Change Impact Analysis in Product-Line Architectures.

ECSA '11: Proceedings of the 5th European Conference on Software Architecture, volume 6903/2011 of Lecture Notes in Computer Science (pp.114-129). Berlin, Heidelberg: Springer-Verlag 2011

[DUEÑ 05] J.C. Dueñas and R. Capilla.

The Decision View of Software Architecture.

Proceedings of the 2nd European Workshop on Software Architecture (EWSA 2005), LNCS 3527. Page(s): 222-230, Springer-Verlag, 2005.

[FALE 13] D. Falessi, L. C. Briand, G. Cantone, R. Capilla and P. Kruchten. The Value of Design Rationale Information.

ACM Transactions on Software Engineering and Methodology (TOSEM) -In memoriam, fault detection and localization, formal methods, modeling and design, Volume 22 Issue 3, 2013.

[FARE 07] R. Farenhorst, P. Lago and H. van Vliet.

EAGLE: Effective Tool Support for Sharing Architectural Knowledge. International Journal of Cooperative Information Systems Vol. 16, Nos. 3 \& 4. Page(s): 413-437, World Scientific Publishing Company. 2007.

[FENT 14] N. Fenton and J. Bieman.

Software Metrics: a Rigorous and Practical Approach. Third Edition CRC Press. 2014.

[FOWL 99] M. Fowler and K. Beck.

Refactoring: Improving the Design of Existing Code

ISBN-13: 978-0201485677. Addison-Wesley, 1999 
[GAMM 95] E. Gamma, R. Helm, R. Johnson and J. Vlissides. Design Patterns: Elements of Reusable Object-Oriented Software. Addison-Wesley Publishing Co., ISBN: 0201633612, 1995.

[GARC 09] J. Garcia, D. Popescu, G. Edwards and N. Medvidovic. Identifying Architectural Bad Smells.

Software Maintenance and Reengineering, 2009. CSMR '09. $13^{\text {th }}$. European Conference on. Page(s): 255-258. 2009.

[GARL 97] D. Garlan, R.T. Monroe and D. Wile. Acme: Architectural Description of Component-Based Systems. CASCON '97 Proceedings of the 1997 conference of the Centre for Advanced Studies on Collaborative research. IBM Press 1997.

[GERD 14] S. Gerdes, S. Lehnert and M. Riebisch. Combining Architectural Design Decisions and Legacy System Evolution.

Proceedings 8th European Conference, ECSA 2014, Vienna, Austria, August 25-29, 2014 LNCS 8627. Page(s): 50-57, 2014. Springer 2014.

[GERD 15] S. Gerdes, M. Soliman and M. Riebisch

Decision Buddy: Tool Support for Constraint-Based Design Decisions during System Evolution.

In Proceedings of the 1st International Workshop on Future of Software Architecture Design Assistants (FoSADA '15). ACM. Page(s), 13-18. 2015.

[GERD 16] S. Gerdes, S. Jasser, M. Riebisch, S. Schröder, M. Soliman, and T Stehle. Towards the essentials of architecture documentation for avoiding architecture erosion.

In Proccedings of the 10th European Conference on Software Architecture Workshops (ECSAW '16). ACM. Article 8, 4 pages. 2016

[GILS 11] F. Gilson and V. Englebert.

Rationale, Decisions and Alternatives Traceability for Architecture Design.

Proceedings of the 5th European Conference on Software Architecture, ECSA'11 Workshop Traceability, Dependencies and Software Architecture, TDSA'11 September 13, 2011. Article 4, 9 pages. ACM 2011.

[HALS 77] M. H. Halstead Elements of Software Science Vol. 7, p. 127. Elsevier. 1997.

[HEES 12] U. van Heesch, P. Avgeriou and R. Hilliard. A Documentation Framework for Architecture Decisions. Journal of Systems and Software, Vol. 85, Issue 4 (April 2012). Page(s): 795-820 Elsevier 2012. 
[ISAA 13] S. Isaac and R. Navon

A graph-based model for the identification of the impact of design changes.

Automation in Construction, Vol. 31, Page(s): 31-40. Elsevier 2013

[ISO 42010:11] ISO/IEC/IEEE Systems and software engineering -- Architecture description.

In ISO/IEC/IEEE 42010:2011(E). 2011.

[ISO 25010:11] ISO/IEC 25010:2011: Systems and Software Engineering Systems and Software Quality Requirements and Evaluation (SQuaRE) System and software quality models. 2011.

[JANS 04] J. Jansen y J. Bosch

Evaluation of Tool Support for Architectural Evolution.

In Proceedings of the 19th IEEE International Conference on Automated Software Engineering (ASE). Page(s): 375-378. IEEE, September 2004

[JANS 05] J. Jansen and J. Bosch.

Software Architecture as a Set of Architectural Design Decisions. Proceedings of the 5th Working IEEE/IFIP Conference on Software Architecture (WICSA'05) Page(s): 109-120, ISBN: 0-7695-2548-2, 2005.

[JANS 07] J. Jansen, J. van der Ven, P. Avgeriou and D. K. Hammer. Tool Support for Architectural Decisions. Proceedings of the Sixth Working IEEE/IFIP Conference on Software Architecture (WICSA 07), ISBN: 0-7695-2744-2, 2007.

[JANS 08A] A. Jansen, T.de Vries, P. Avgeriou and M. van Veelen. Sharing the Architectural Knowledge of Quantitative Analysis. Proceedings of the Quality of Software-Architectures (QoSA 2008). Page(s): 200-234. Springer Berlin Heidelberg. 2008.

[JANS 08B] A. Jansen.

Architectural Design Decisions. Doctoral Dissertation. University of Groningen, Institute for Mathematics and Computing Science. August 2008. ISBN: 978-90-367-3494-3. 2008.

[KAN 02] S. H. Kan.

Metrics and Models in Software Quality Engineering. Addison-Wesley Longman Publishing Co., Inc. 2002.

[KOZI 11] H. Koziolek.

Sustainability Evaluation of Software Architectures: a Systematic Review.

Proceedings of the joint ACM SIGSOFT conference --QoSA and ACM SIGSOFT symposium --ISARCS on Quality of software architectures -QoSA and architecting critical systems --ISARCS Page(s): 3-12. ISBN: 978-1-4503-0724-6. ACM 2011. 
[KOZI 13] H. Koziolek, D. Domis, T. Goldschmidt and P. Vorst. Measuring Architecture Sustainability.

IEEE Software November/December (4). Page(s): 54-62. 2013.

[KRUC 95] P. Kruchten.

Architectural Blueprints -The 4+1 View Model of Software Architecture IEEE Software 12 (6). Page(s): 42-50, 1995.

[KRUC 04] P. Kruchten.

An Ontology of Architectural Design Decisions in Software Intensive Systems.

In 2nd Groningen Workshop on Software Variability. Page(s): 54-61, 2004.

[KRUC 06] P. Kruchten, P. Lago and H. van Vliet.

Building up and Reasoning about Architectural Knowledge.

2nd International Conference on Quality of Software Architectures

(QoSA). Page(s): 43-58, 2006.

[KRUC 09] P. Kruchten, R. Capilla and J.C. Dueñas.

The Decision View's Role in Software Architecture Practice.

IEEE Software Published by the IEEE Computer Society Page(s): 36-42, 2009.

[KRUC 12A] P. Kruchten, R.L. Nord, I. Ozkaya.

Technical Debt: From Metaphor to Theory and Practice.

IEEE Software 29(6) Page(s): 18-21, 2012.

[KRUC 12B] P. Kruchten.

Strategic Management of Technical Debt: Tutorial Synopsis.

2012 12th International Conference on Quality Software (QSIC),

Page(s): 282-284. IEEE. 2012.

[LAGO 06] P. Lago and P. Avgeriou.

First workshop on sharing and reusing architectural knowledge.

SIGSOFT Software Engineering Notes, 31(5) Page(s): 32-36, 2006.

[LAGO 09] P. Lago, H. Muccini and H. van Vliet.

A Scoped Approach to Traceability Management.

Journal of Systems and Software Vol. 82. Issue 1. Page(s): 168-182.

Elsevier. 2009.

[LAGO 15] P. Lago, S. A. Koçak, I. Crnkovic and B. Penzenstadler.

Framing Sustainability as a Property of Software Quality.

Communications of the ACM 58(10)10. Page(s): 70-78. 2015. 
[LE 16] D. M. Le, C. Carrillo, R. Capilla and N. Medvidovic, Relating Architectural Decay and Sustainability of Software Systems, 2016 13th Working IEEE/IFIP Conference on Software Architecture (WICSA), Venice, 2016, pp. 178-181. 2016

[LEE 04] L. Lee and P. Kruchten.

Customizing the Capture of Software Architectural Design Decisions. University of British Columbia. 2004.

[LI 12] B. Li, X. Sun, H. Leung and S. Zhang. A Survey of Code-Based Change Impact Analysis Techniques. Software Testing, Verification and Reliability, 2012.

[LI 14] Z. Li, P. Liang and P. Avgeriou. Architectural Debt Management in Value-Oriented Architecting. In Econimics-Driven Software Architecture, I. Mistrik, R. Bahsoon, R. Kazman and Y. Zhang (Eds),

Elsevier. Page(s): 183-204. 2014.

[LI 15] Z. Li, P. Liang and P. Avgeriou.

Architectural Technical Debt Identification Based on Architecture Decisions and Change Scenarios.

In Software Architecture (WICSA), 2015 12th Working IEEE/IFIP Conference on. Page(s): 65-74. IEEE. 2015

[LI 15B] Zengyang Li, Paris Avgeriou, and Peng Liang. 2015.

A systematic mapping study on technical debt and its management. J. Syst. Softw. 101, C (March 2015), Page(s): 193-220. 2015

[LI 16] B. Li, L. Liao and J. Si.

A Technique to Evaluate Software Evolution Based on Architecture Metric.

2016 IEEE 14th International Conference on Software Engineering Research, Management and Applications (SERA), Page(s): 1-8. Towson, MD, 2016

[LIPP 06] M. Lippert and S. Roock.

Refactoring in Large Software Projects: Performing Complex

Restructurings Successfully.

John Wiley \& Sons. 2006.

[LOSA 04] F. Losavio, L. Chirinos, A. Matteo, N. Lévy and A. Ramdane-Cherif, ISO Quality Standards for Measuring Architectures, Journal of Systems and Software, Volume 72, Issue 2, July 2004, Page(s): 209-223, ISSN 0164-1212. 2004

[LYTR 12A] I. Lytra, S. Sobernig, U. Zdun.

Architectural Decision Making for Service-Based Platform Integration: A Qualitative Multi-Method Study.

WICSA/ECSA IEEE DL 111-120, 2012. 
[LYTR 12B] I. Lytra, S. Sobernig, H. Tran, U. Zdun.

A pattern language for service-based platform inte-gration and adaptation.

17th European Conference on Pattern Languages of Programs, EuroPLoP 2012 ACM DL 4:1-4:27, 2012.

[LYTR 13] I. Lytra, H. Tran and U. Zdun.

Supporting Consistency between Architectural Design Decisions and Component Models through Reusable Architectural Knowledge Transformations.

In Proceedings of the 7th European Conference on Software Architecture (ECSA'13). Page(s): 224-239. Springer 2013.

[LYTR 15A] I. Lytra, G. Engelbrecht, D. Schall and U. Zdun.

Reusable Architectural Decision Models for Quality-driven Decision Support: A Case Study from a Smart Cities Software Ecosystem. In 2015 IEEE/ACM 3rd International Workshop on Software Engineering for Systems-of-Systems (SESoS), Page(s): 37-43. 2015

[LYTR 15B] I. Lytra, P. Gaubatz, and U. Zdun.

Two Controlled Experiments on Model-Based Architectural Decision making.

Journal Information and Software Technology, Vol. 63, Issue C, July 2015. Page(s): 58-75. 2015

[LOPE 15] I. C. Lopes, P. H. S. Brito, B. F. dos S. Neto, E. Costa and A. Almeida. A Decision-Making Tool to Support Architectural Designs Based on Quality Attributes.

In Proceedings of the 30th Annual ACM Symposium on Applied Computing (SAC '15). ACM. Page(s): 1457-1463. 2015.

[MADE 12] P. Mäder and O. Gotel.

Towards Automated Traceability Maintenance.

Elsevier, the Journal of Systems and Software. Volume 85, Issue 10, October 2012. Page(s): 2205-2227. 2012.

[MAHA 12] M. Mahaux and C. Canon.

Integrating the Complexity of Sustainability in Requirements

Engineering.

In First International Workshop on Requirements for Sustainable

Systems. 2012.

[MALA 11] I. Malavolta, H. Muccini and S. Rekha.

Supporting Architectural Design Decisions Evolution through Model Driven Engineering.

Springer. Page(s): 63-77, 2011. 
[MALA 14] I. Malavolta, H. Muccini and S. Rekha.

Enhancing Architecture Design Decisions Evolution with Group Decision Making Principles.

In Software Engineering for Resilient Systems Page(s): 9-23. Springer International Publishing. 2014.

[MANT 14] C. Manteuffel, D. Tofan, H. Koziolek, T. Goldschmidt and P. Avgeriou. Industrial Implementation of a Documentation Framework for Architectural Decisions.

2014 IEEE/IFIP Conference on Software Architecture WICSA. Page(s): 225-234, 2014.

[MANT 16] C. Manteuffel, D. Tofan, P. Avgeriou, H. Koziolek and T.Goldschmidt. Decision Architect - A decision Documentation Tool for Industry. Journal of Systems and Software, 112. Page(s): 181-198. 2016.

[MART 94] R. Martin

OO design quality metrics: An analysis of dependencies, Proc. Workshop Pragmatic and Theoretical Directions in Object-Oriented Software Metrics, OOPSLA’94, 1994

[MART 15] A. Martini and J. Bosch.

The Danger of Architectural Technical Debt: Contagious Debt and Vicious Circles.

2015 12th Working IEEE/IFIP Conference on Software Architecture (WICSA). Page(s) 1-10, May 2015

[MCCA 76] T.J. McCabe

A complexity measure.

IEEE Transactions on software Engineering, (4), 308-320. 1976

[MIRA 11A] M. Mirakhorli.

Tracing Architecturally Significant Requirements: A Decision-Centric Approach.

33th International Conference on Software Engineering (ICSE), Honolulu, Hawaii, USA, May 2011

[MIRA 11B] M. Mirakhorli and J. Cleland-Huang. Transforming Trace Information in Architectural Documents into Reusable and Effective Traceability. in Proceedings of the Sixth Workshop on SHAring and Reusing architectural Knowledge, ICSE 2011, Honolulu, Hawaii, USA, May 2011

[MORA 96] Thomas P. Moran and John M. Carroll (Eds.).

Design Rationale: Concepts, Techniques, and Use.

L. Erlbaum Assoc. Inc., Hillsdale, NJ, USA. 1996 
[MUCC 15] H. Muccini, D.A. Tamburri and V.S. Rekha. On the Social Dimensions of Architectural Decisions. In European Conference on Software Architecture ECSA 2015. Page(s) 137-145. Springer International Publishing. 2015

[NAUM 11] S. Naumann, M. Dick, E. Kern and T. Johann. The GREENSOFT Model: A Reference Model for Green and Sustainable Software and its Engineering.

Sustainable Computing: Informatics and Systems 1 (2011) Page(s): 294304, Elsevier 2011. 33th International Conference on Software Engineering (ICSE), Honolulu, Hawaii, USA, May 2011. ACM 978-14503-0445-0/11/05. 2011.

[NORD 14] R. L. Nord, I. Ozkaya, H. Koziolek and P. Avgeriou. Quantifying Software Architecture Quality Report on the First International Workshop on Software Architecture Metrics.

ACM SIGSOFT Software Engineering Notes, Vol 39, Issue 5. Page(s): 32-34. 2014.

[NOWA 13] M. Nowak and C. Pautasso.

Team Situational Awareness and Architectural Decision Making with the Software Architecture Warehouse.

In Proceedings of the 7th European conference on Software Architecture (ECSA'13), Khalil Drira (Ed.). Springer-Verlag, Berlin, Heidelberg. Page(s): 146-161. 2013.

[OH 14] J. Oh and S. Kang.

A Hierarchical Model for Traceability between Requirements and Architecture.

SAC'14, Proceedings of the 29th Annual ACM Symposium on Applied Computing. March 24-28, 2014, Gyeongju, Korea. ACM 978-1-45032469-4/14/03. Page(s): 1035-1042. 2014.

[OZKA 15] I. Ozkaya, R.L. Nord, H. Koziolek and P. Avgeriou.

Toward Simpler, not Simplistic, Quantification of Software Architecture and Metrics: Report on the Second International Workshop on Software Architecture and Metrics.

ACM SIGSOFT Software Engineering Notes, 40(5) Page(s): 43-46. 2015.

[PARN 89] D. Parnas, P.C. Clements and D.M. Weiss. Enhancing Reusability with Information Hiding. In Software Reusability. Concepts and Models, Vol. I. Page(s): 141-157, ACM Press, ISBN: 0-201-50018-3, 1989.

[PERR 92] D.E. Perry and A.L. Wolf.

Foundations for the Study of Software Architecture.

ACM SIGSOFT Software Engineering Notes, 17(4). Page(s): 40-52, 1992. 
[POTT 88] N. Rozanski and E. Woods.

Recording the Reasons for Design Decisions.

MCC Software Technology Program, IEEE 1988.

[RAZA 14] M. Razavian, G. Procaccianti and D.A. Tamburri

Four-Dimensional Sustainable e-services.

In Proceedings of the International Conference on Informatics for

Environmental Protection. Page(s): 221-228. 2014.

[RAZA 16] M. Razavian, A. Tang, R. Capilla and P. Lago.

In two minds: how reflections influence software design thinking.

J. Softw. Evol. Process Vol. 28, Issue 6, Page(s): 394-426. 2016

[RIEB 07] M. Riebisch and S. Wohlfarth.

Introducing Impact Analysis for Architectural Decisions.

14th Annual IEEE International Conference and Workshops on the

Engineering of Computer-Based Systems, ECBS'07. Page(s) 381-392.

IEEE 2007.

[ROZA 05] N. Rozanski and E. Woods.

Software Systems Architecture: Working with Stakeholders Using

Viewpoints and Perspectives.

Boston, MA: Addison-Wesley, 2005.

[SALA 16A] M. Salama and R. Bahsoon

Proceeding SAC '16 Proceedings of the 31st Annual ACM Symposium on Applied Computing Page(s): 1354-1357. 2016.

[SALA 16B] M. Salama, A. Shawish and R. Bahsoon.

Dynamic Modelling of Tactics Impact on the Stability of Self-Aware

Cloud Architectures.

2016 IEEE 9th International Conference on Cloud Computing (CLOUD),

San Francisco, CA, Page(s) 871-875. 2016

[SCHU 07] N. Schuster, O. Zimmermann and C. Pautasso

ADkwik: Web 2.0 Collaboration System for Architectural Decision

Engineering.

In SEKE. Page(s). 255-260. 2007

[SCHW 13] R. Schwanke, L. Xiao and Y. Cai.

Measuring Architecture Quality by Structure Plus History Analysis.

Proceedings of the 2013 International Conference on Software

Engineering (ICSE '13). IEEE Press. Page(s): 891-900. 2013.

[SEHE 14] S. Sehestedt, D. H. Cheng and E. Bouwers

Towards Quantitative Metrics for Architecture Models.

In Proceedings of the WICSA 2014 Companion Volume (p. 5). ACM.

2014 
[SETH 09] K. Sethi, Y. Cai, S. Wong, A. Garcia and C. Sant'Anna.

From Retrospect to Prospect: Assessing Modularity and Stability from Software Architecture.

In Software Architecture, 2009 \& European Conference on Software Architecture. WICSA/ECSA 2009. Joint Working IEEE/IFIP Conference on. Page(s): 269-272. IEEE. 2009.

[SHAW 96] M. Shaw and D. Garlan.

Software Architecture. Perspectives on an Emerging Discipline.

Prentice-Hall, ISBN: 0-13-182957-2, 1996.

[SHAW 97] M. Shaw and P. Clements.

A Field Guide to Boxology: Preliminary Classification of Architectural Styles for Software Systems.

In Proceedings of the Twenty-First Annual International Computer Software and Applications Conference (COMPSAC'97) Page(s): 6-13, Washington, D.C., Aug. 1997.

[SHUK 12] V. Shukla, G. Auriol and C. Baron.

Integrated Requirement Traceability, Multiview Modelling and DecisionMaking. A systems Engineering Approach for Integrating Processes and Product.

Systems Conference (SysCon), ISBN: 978-1-4673-0748-2. IEEE 2012.

[SNIP 12] W. Snipes, B. Robinson, Y. Guo and C. Seaman.

Defining the decision factors for managing defects: a technical debt perspective.

In Proceedings of the Third International Workshop on Managing Technical Debt (MTD '12). IEEE Press, Piscataway, NJ, USA. Page(s): 54-60. 2012

[SOMM 10] I. Sommerville.

Software engineering. - 9th ed.

Addison-Wesley Publishing Company, US. 2010

[SONI 95] D. Soni, R.L. Nord and C. Hofmeister.

Software Architecture in Industrial Applications.

In International Conference on Software Engineering. Page(s): 196-207, 1995.

Addison-Wesley. ISBN-10: 0-13-703515-2. 2011.

[STEV 16] S. Stevanetic, K. Plakidas, T. B. Ionescu, D. Schall, and U. Zdun.

Supporting quality-driven architectural design decisions in software ecosystems.

In Proccedings of the 10th European Conference on Software Architecture Workshops (ECSAW '16). ACM, Article 22, 4 pages. 2016. 
[SPAN 05] G. Spanoudakis and A. Zisman.

Software Traceability: a Roadmap.

Handbook Engineering and Knowledge Engineering Vol III: Recent Advancements. World Scientific Publishing Co. ISBN 981-256-273-7, 2005.

[TANG 05] A. Tang and J. Han.

Architecture Rationalization: A Methodology for Architecture Verifiability, Traceability and Completeness.

12th Annual IEEE International Conference and Workshop on the Engineering of Computer Based Systems ECBS 2005.

[TANG 07] A. Tang, A. E. Nicholson, Y. Jin and J. Han, Using Bayesian Belief Networks for Change Impact Analysis in Architecture Design Journal of Systems and Software, vol. 80, no. 1. Page(s): 127-148, Jan. 2007.

[TANG 11] A. Tang, P. Liang, V. Clerc and H. van Vliet. Traceability in the Co-evolution of Architectural Requirements and Design.

Relating Software Requirements and Architectures. Springer. Page(s): 35-60, ISBN: 978-3-642-21000-6, 2011.

[TYRE 05] J. Tyree and A. Akerman.

Architecture Decisions: Demystifying Architecture.

IEEE Software, vol. 22, no 2, Page(s): 19-27, 2005.

[VENT 13] C. C. Venters, L. Lau, M. K. Griffiths, V. Holmes, R. R. Ward, C. Jay, C. E. Dibsdale and J. Xu

The Blind men and the elephant: Towards an Empirical Evaluation Framework for Software Sustainability.

WSSSPE'1: First Workshop On Sustainable Software For Science: Practice and Experiences, SC'13, 17 November 2013, Denver, CO, USA, 2013.

[VILL 16] L. Villa, I, Cabezas, M. Lopez, and O. Casas.

Towards a Sustainable Architectural Design by an Adaptation of the Architectural Driven Design Method.

In International Conference on Computational Science and Its Applications. Page(s): 71-86. Springer International Publishing. 2016

[VVEN 06] J. S. van der Ven, A. G. J. Jansen, J. A. G. Nijhuis and J. Bosch. Design Decisions: The Bridge between Rationale and Architecture. Rationale management in software engineering. Page(s): 329-348. Springer 2006.

[VGUR 02] J. van Gurp and J. Bosch. Design Erosion: Problems \& causes. Journal of Systems \& Software, 61(2). Page(s): 105-119, march 2002. 
[ZDUN 13] U. Zdun, R. Capilla, H. Tran and O. Zimmermann. Sustainable Architectural Design Decisions. IEEE Software November/December (4). Page(s): 46-53. 2013.

[ZIMM 07] O. Zimmerman, T. Gschwind, J. Küster, F. Leymann and N. Schuster. Reusable Architectural Decision Models for Enterprise Application Development.

Springer-Verlag Berlin Heidelberg 2007.

[ZIMM 11] O. Zimmermann.

Architectural Decisions as Reusable Design Assets.

WICSA/ECSA 2012. August 20-24, Helsinki, Finland. 2012.

[ZIMM 15A] O. Zimmermann, L. Wegmann, H. Koziolek and T. Goldschmidt. Architectural Decision Guidance Across Projects - Problem Space Modeling, Decision Backlog Management and Cloud Computing Knowledge.

Software Architecture (WICSA), 2015 12th Working IEEE/IFIP Conference on, Montreal, QC, 2015. Page(s): 85-94. 2015.

[ZIMM 15B] O. Zimmermann.

Metrics for Architectural Synthesis and Evaluation -- Requirements and Compilation by Viewpoint. An Industrial Experience Report.

2015 IEEE/ACM 2nd International Workshop on Software Architecture and Metrics (SAM). Page(s): 8-14, 2015.

[@ADvISE] Architectural Design Decision Support Framework (ADvISE) https://swa.univie.ac.at/Software_Architecture/researchprojects/architectural-design-decision-support-framework-advise/ November, 2016

[@HIPE] Hipergate. An open source CRM and Groupware system. http://www.hipergate.com , 05/07 


\section{Anexo I. Atributos de ADDs propuestos por Tyree}

En este anexo detallamos los atributos propuestos por Tyree [TYRE 05] para la gestión de las decisiones de diseño.

Tabla I.1. Plantilla de atributos propuestos.

\begin{tabular}{|c|c|}
\hline Atributo & Descripción \\
\hline Problema & $\begin{array}{l}\text { Describe el diseño arquitectónico que tratamos de abordar y las razones de } \\
\text { por qué debe realizarse. }\end{array}$ \\
\hline Decisión & $\begin{array}{l}\text { Representa la decisión que claramente indica la dirección para construir una } \\
\text { determinada arquitectura. }\end{array}$ \\
\hline Estado & Especifica el estado en el que se encuentra una la decisión. \\
\hline Grupo & $\begin{array}{l}\text { El grupo en que se clasifica la decisión. Las ontologías pueden utilizarse como } \\
\text { medio de clasificación. }\end{array}$ \\
\hline Asunciones & $\begin{array}{l}\text { Describen las asunciones subyacentes en el entorno en que se ha tomado la } \\
\text { decisión. }\end{array}$ \\
\hline Restricciones & $\begin{array}{l}\text { Son las limitaciones que se imponen en el espacio de la solución para las } \\
\text { decisiones tomadas. }\end{array}$ \\
\hline Posiciones & $\begin{array}{l}\text { Muestra una lista de posiciones (alternativas) consideradas y que } \\
\text { generalmente requieren de largas explicaciones. Sólo aquellas que se } \\
\text { consideran viables deben ser descritas. }\end{array}$ \\
\hline Argumento & Describe por qué se ha seleccionado una determinada posición o alternativa. \\
\hline Implicaciones & Describe las implicaciones de tomar una decisión determinada. \\
\hline $\begin{array}{l}\text { Decisiones } \\
\text { relacionadas }\end{array}$ & Son relaciones que se establecen entre las distintas decisiones. \\
\hline $\begin{array}{l}\text { Requisitos } \\
\text { relacionados }\end{array}$ & $\begin{array}{l}\text { Lista de los requisitos que han influido en la decisión ya que estas son } \\
\text { mayormente dirigidas por objetivos de negocio. }\end{array}$ \\
\hline $\begin{array}{l}\text { Artefactos } \\
\text { relacionados }\end{array}$ & Lista de las arquitecturas relacionadas con la decisión. \\
\hline $\begin{array}{l}\text { Principios } \\
\text { relacionados }\end{array}$ & $\begin{array}{l}\text { Lista de los principios tenidos en cuenta para tomar la decisión, generalmente } \\
\text { aquellos relacionados con la empresa a los cuales las decisiones deben } \\
\text { adherirse. }\end{array}$ \\
\hline Notas & Notas que se pueden tomar durante la captura de decisiones. \\
\hline
\end{tabular}




\section{Anexo II. Atributos de ADDs propuestos por Capilla}

En este anexo detallamos los atributos propuestos Capilla [CAPI 07A] (tablas II.1, II.2 y II.3) [CAPI 07B] (tablas II.4 y II.5) para la gestión de las decisiones de diseño.

Tabla II.1. Plantilla de atributos obligatorios.

\begin{tabular}{|c|c|}
\hline Atributo & Descripción \\
\hline Nombre & Contiene el nombre de la decisión. \\
\hline $\begin{array}{l}\text { Descripción de la } \\
\text { decisión }\end{array}$ & Contiene la descripción de la decisión. \\
\hline Restricciones & Influye en la viabilidad de la decisión. \\
\hline Dependencias & $\begin{array}{l}\text { Las decisiones pueden establecer dependencias con decisiones tomadas } \\
\text { anteriormente. }\end{array}$ \\
\hline Estado & $\begin{array}{l}\text { Refleja el estado de la decisión. Puede ser pendiente, rechazada, aprobada u } \\
\text { obsoleta. }\end{array}$ \\
\hline Lógica & Describe los motivos o razones que justifican una decisión. \\
\hline Patrones de diseño & $\begin{array}{l}\text { Son elementos de conocimiento arquitectónico suficientemente conocidos } \\
\text { que se usan para tomar decisiones de diseño. }\end{array}$ \\
\hline Arquitectura & $\begin{array}{l}\text { Representa la arquitectura software como el resultado de un conjunto de } \\
\text { decisiones de diseño. }\end{array}$ \\
\hline Requisitos & $\begin{array}{l}\text { Son los requisitos que motivan las decisiones de diseño y con las cuales se } \\
\text { relacionan. }\end{array}$ \\
\hline
\end{tabular}


Anexo II. Atributos de ADDs propuestos por Capilla

Tabla II.2. Plantilla de atributos opcionales.

\begin{tabular}{|c|c|}
\hline Atributo & Descripción \\
\hline $\begin{array}{l}\text { Decisiones } \\
\text { alternativas }\end{array}$ & $\begin{array}{l}\text { Son aquellas que se han considerado como una opción válida a la decisión } \\
\text { tomada. }\end{array}$ \\
\hline Hipótesis & Permiten conocer los fundamentos en los que se basa la toma de decisiones. \\
\hline $\begin{array}{l}\text { A favor y en } \\
\text { contra }\end{array}$ & Se corresponde con la evaluación de las decisiones alternativas. \\
\hline Categoría & $\begin{array}{l}\text { Clasifican las decisiones en diferentes categorías acorde a los intereses de } \\
\text { una organización particular o individuo. }\end{array}$ \\
\hline Iteración & $\begin{array}{l}\text { Las decisiones se toman a lo largo del proceso iterativo que define la toma } \\
\text { de decisiones, por lo que es necesario organizar dichas decisiones en } \\
\text { iteraciones dentro del proceso de construcción arquitectónico. }\end{array}$ \\
\hline $\begin{array}{l}\text { Información sobre } \\
\text { la arquitectura y } \\
\text { del proyecto }\end{array}$ & Son específicos del proyecto y contienen información de la arquitectura. \\
\hline Responsable & Es la persona que toma las decisiones. \\
\hline $\begin{array}{l}\text { Vista de la } \\
\text { arquitectura }\end{array}$ & Representa la vista de la arquitectura. \\
\hline Arquitectos & $\begin{array}{l}\text { Son aquellos Stakeholders involucrados en el proceso de construcción } \\
\text { arquitectónico y que participan en la toma de decisiones. }\end{array}$ \\
\hline $\begin{array}{l}\text { Principios } \\
\text { relacionados }\end{array}$ & $\begin{array}{l}\text { Es la relación de principios de diseño que pueden ser interesantes en un } \\
\text { momento dado. }\end{array}$ \\
\hline Notas & Información complementaria. \\
\hline $\begin{array}{l}\text { Atributos de } \\
\text { calidad }\end{array}$ & $\begin{array}{l}\text { Atributos que afectan a una o más de una decisión, y que aparecen en } \\
\text { aquellas decisiones influidas por requisitos no funcionales. }\end{array}$ \\
\hline
\end{tabular}


Anexo II. Atributos de ADDs propuestos por Capilla

Tabla II.3. Plantilla de atributos evolutivos.

\begin{tabular}{l|l} 
Atributo & Descripción \\
\hline Fecha & Fecha en la que se toma la decisión. \\
\hline Versión & Identifica una versión de la misma decisión. \\
\hline Obsoleto & Corresponde al estado de aquellas que ya no se consideran vigentes. \\
\hline Validadas & Decisiones que son confirmadas como válidas a lo largo del tiempo. \\
\hline Reutilización & Indica el número de veces que una decisión ha sido utilizada. \\
\hline Clasificación & Determina un orden dentro de la clasificación de decisiones reutilizadas. \\
\hline $\begin{array}{l}\text { Enlaces entre } \\
\text { trazas }\end{array}$ & Relación entre decisiones y requisitos, y entre decisiones y arquitectura. \\
\hline
\end{tabular}

Tabla II.4. Plantilla de atributos evolutivos en las decisiones de diseño.

\begin{tabular}{|c|c|}
\hline Atributo & Descripción \\
\hline Fecha & Fecha en la que se toma la decisión. \\
\hline Versión & Identifica una versión de la misma decisión. \\
\hline Autor & Se corresponde con el autor de la decisión. \\
\hline Estado & Corresponde al estado actual de la decisión. \\
\hline Validada & Decisiones que son confirmadas como válidas a lo largo del tiempo. \\
\hline Tiempo de vida & Determina cuando la decisión podrá ser revisada. \\
\hline Ámbito & Qué elementos pueden ser afectados por una decisión. \\
\hline Razón de evolución & Defina la causa del cambio. \\
\hline $\begin{array}{l}\text { Flag de decisión } \\
\text { derivada }\end{array}$ & Identifica aquellas decisiones tomadas a partir de una ya existente. \\
\hline Será arquitectura & Decisiones planteadas para su uso en un futuro. \\
\hline $\begin{array}{l}\text { Número de decisiones } \\
\text { y enlaces }\end{array}$ & $\begin{array}{l}\text { Proporciona información sobre si la evolución del sistema evoluciona a } \\
\text { una mayor complejidad. }\end{array}$ \\
\hline $\begin{array}{l}\text { Número de cambios } \\
\text { en la decisión }\end{array}$ & Cuantifica cuantas veces ha cambiado una decisión. \\
\hline
\end{tabular}


Anexo II. Atributos de ADDs propuestos por Capilla

Tabla II.5. Plantilla de atributos evolutivos en las relaciones entre decisiones.

\begin{tabular}{|c|c|}
\hline Atributo & Descripción \\
\hline Versión & Incluye la fecha y versión del enlace. \\
\hline Entre decisiones & Identificados por el nombre, fecha, tipo de enlace y su histórico. \\
\hline $\begin{array}{l}\text { Entre requisitos y } \\
\text { objetos de diseño }\end{array}$ & Identificados por el nombre, fecha, tipo de enlace y su histórico. \\
\hline Categoría & $\begin{array}{l}\text { Distingue los tipos depende, requiere, excluye, excluye cuando, caso } \\
\text { especial. }\end{array}$ \\
\hline
\end{tabular}




\section{Anexo III. Diseño de la aplicación $A D D N$-Metrics}

En este anexo reflejamos el diagrama de clases UML (figura IV.1) de la aplicación Architectural Design Decision Network Metrics (ADDN-Metrics) propuesta en este trabajo.

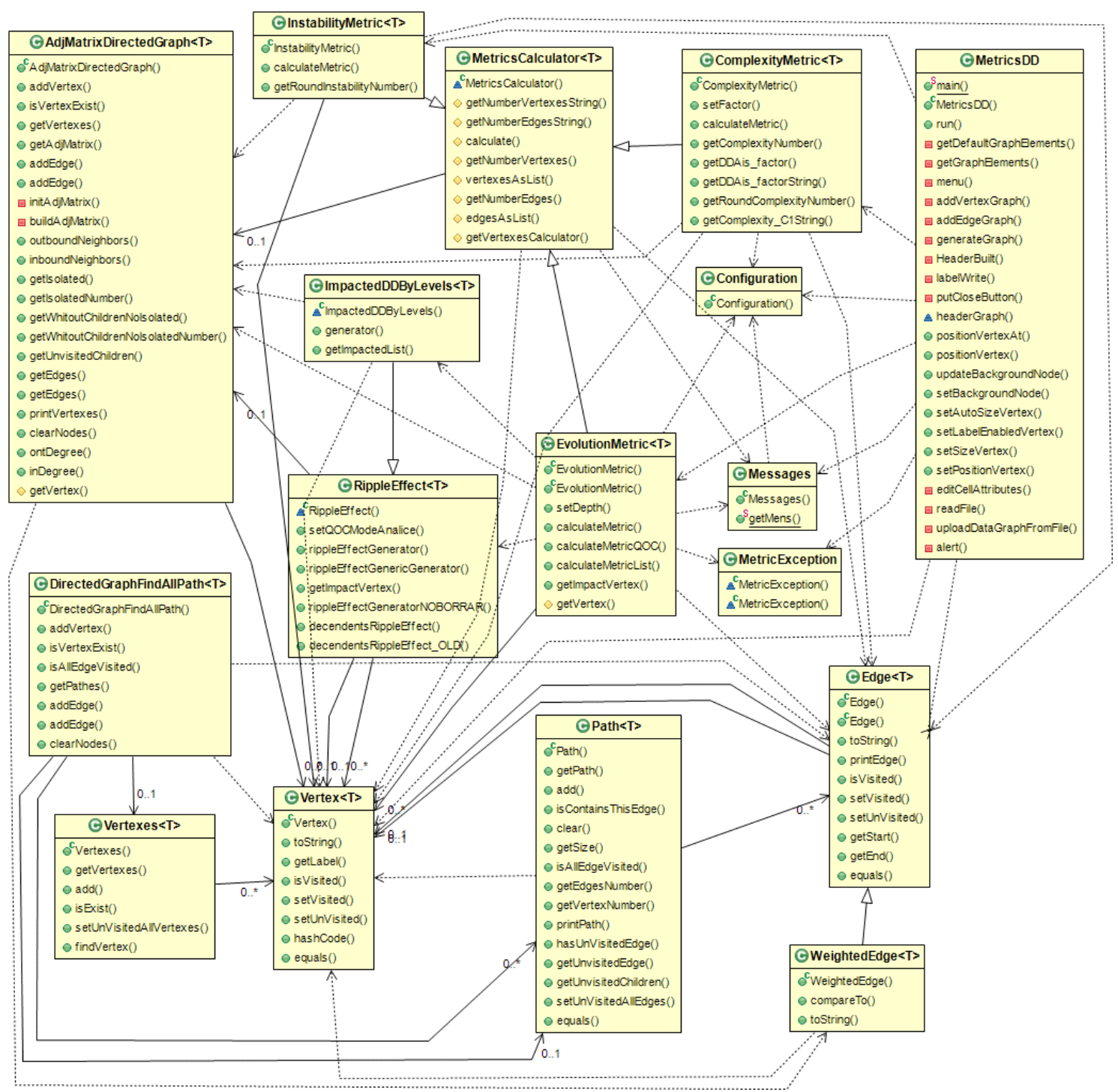

Figura IV.1: Relación de clases UML de la aplicación ADDN-Metrics. 


\section{Anexo IV. Código de la aplicación ADDN-Metrics}

En este anexo indicaremos el código empleado en cada una de las clases POO que forman parte de nuestra aplicación

\section{Clase Configuración.}

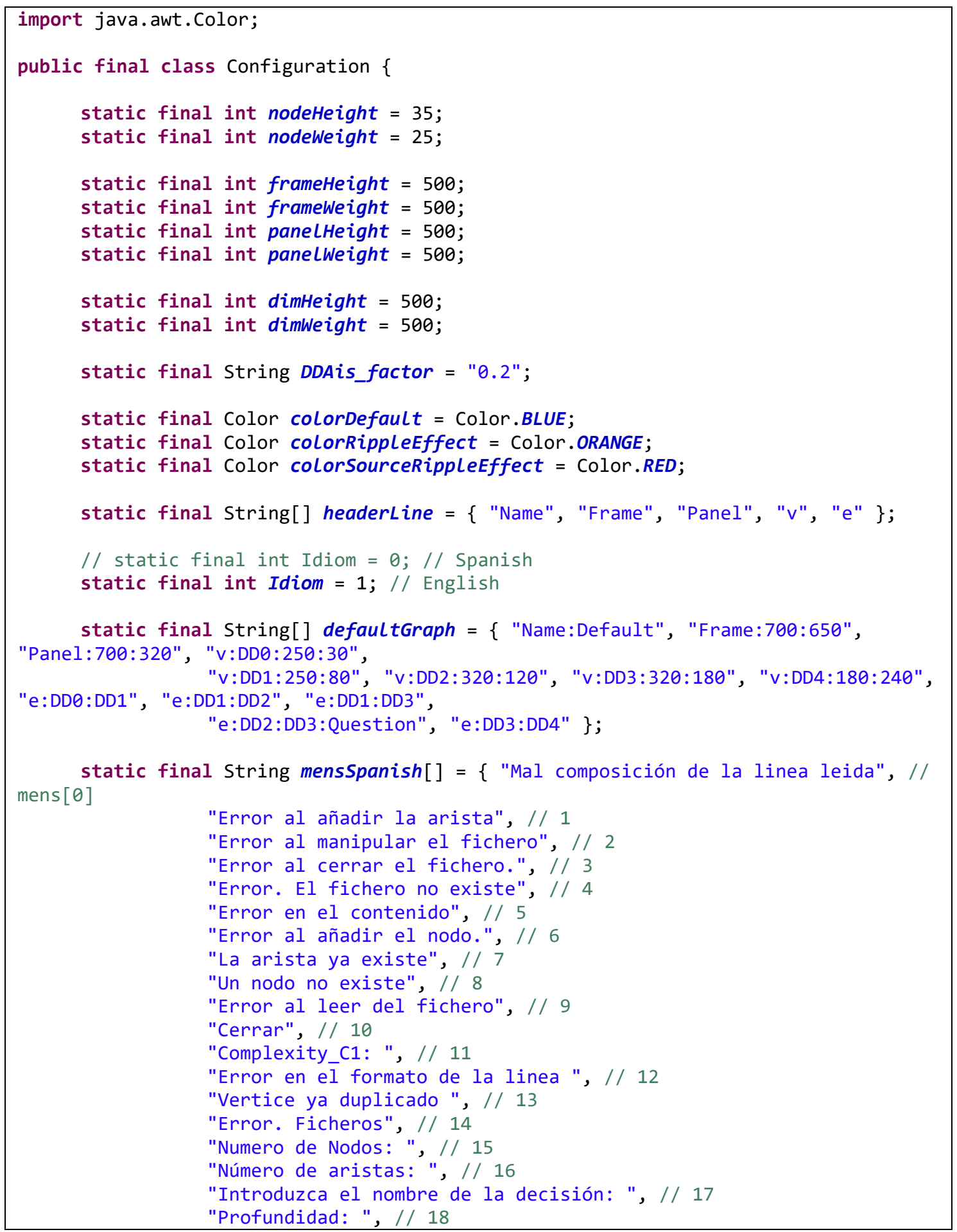




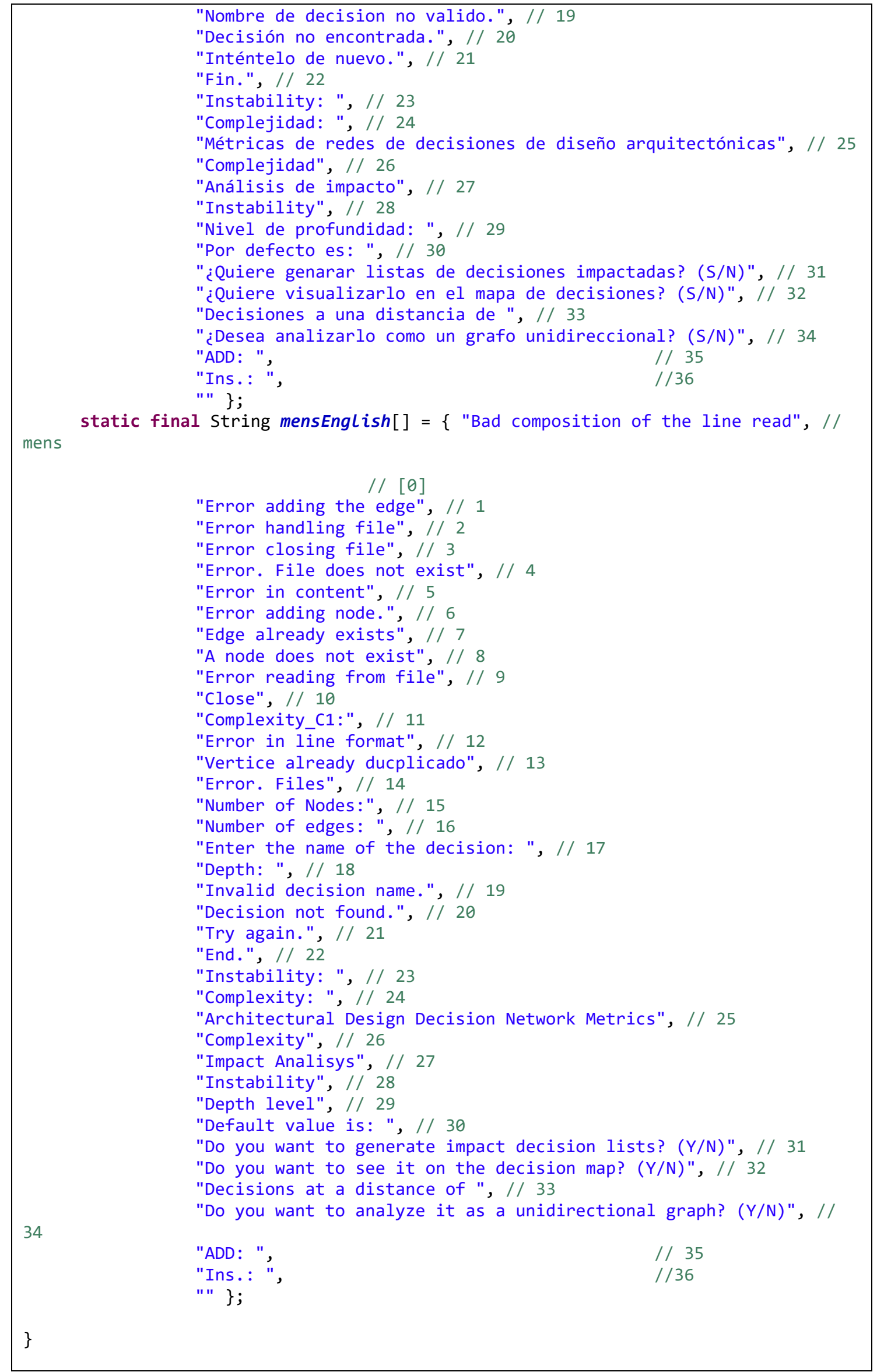




\section{Clase MetricsDD.}

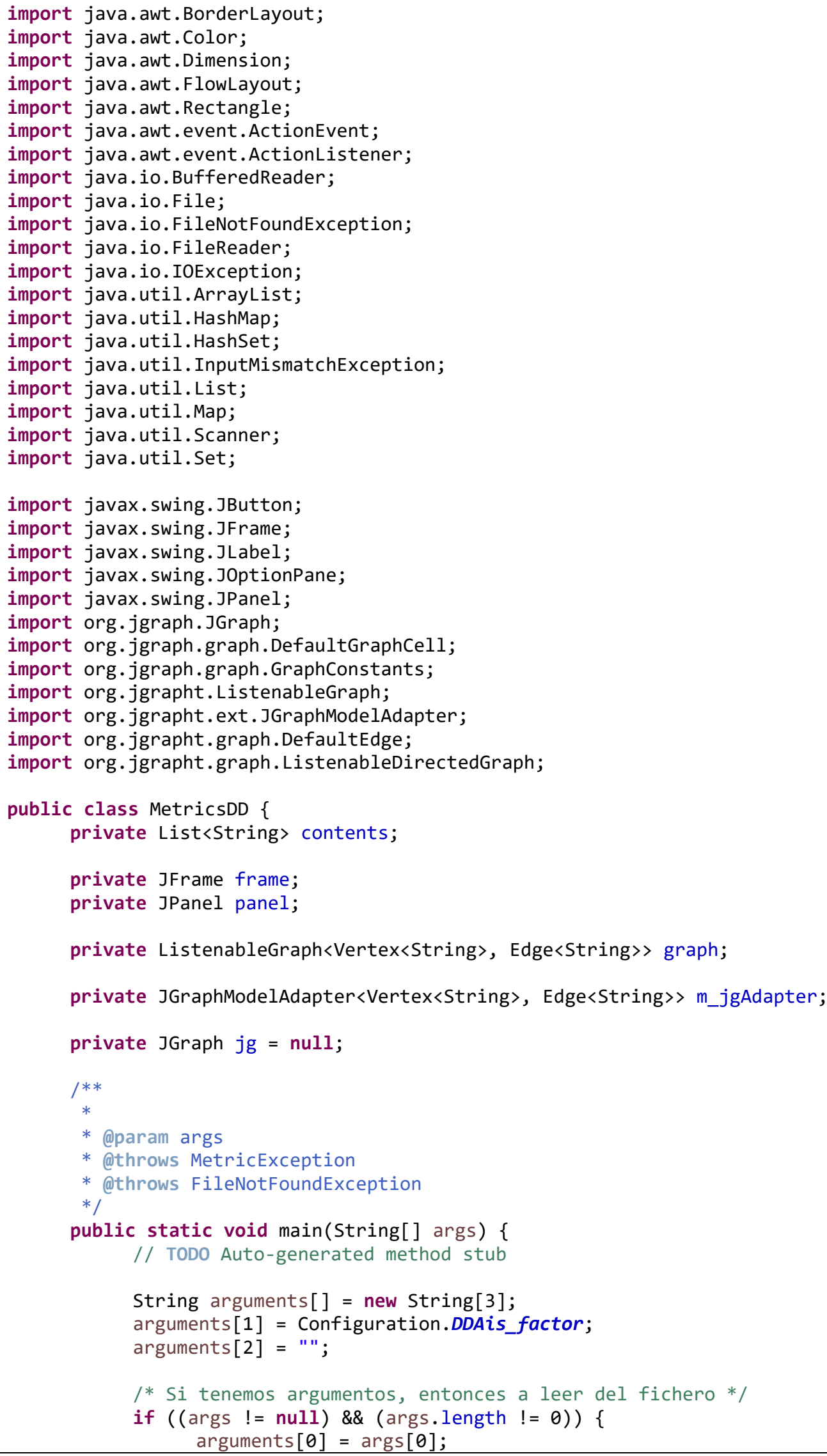




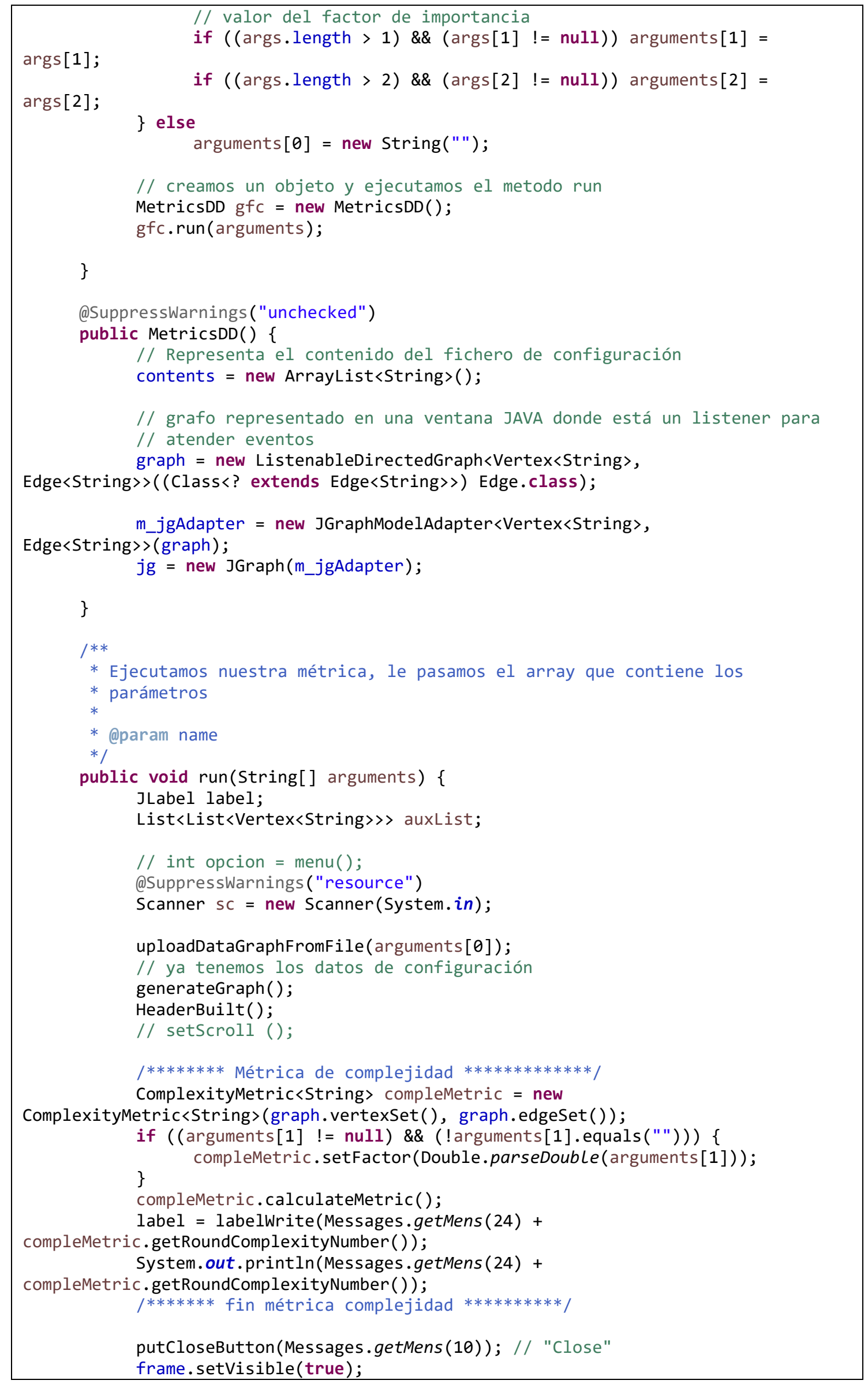




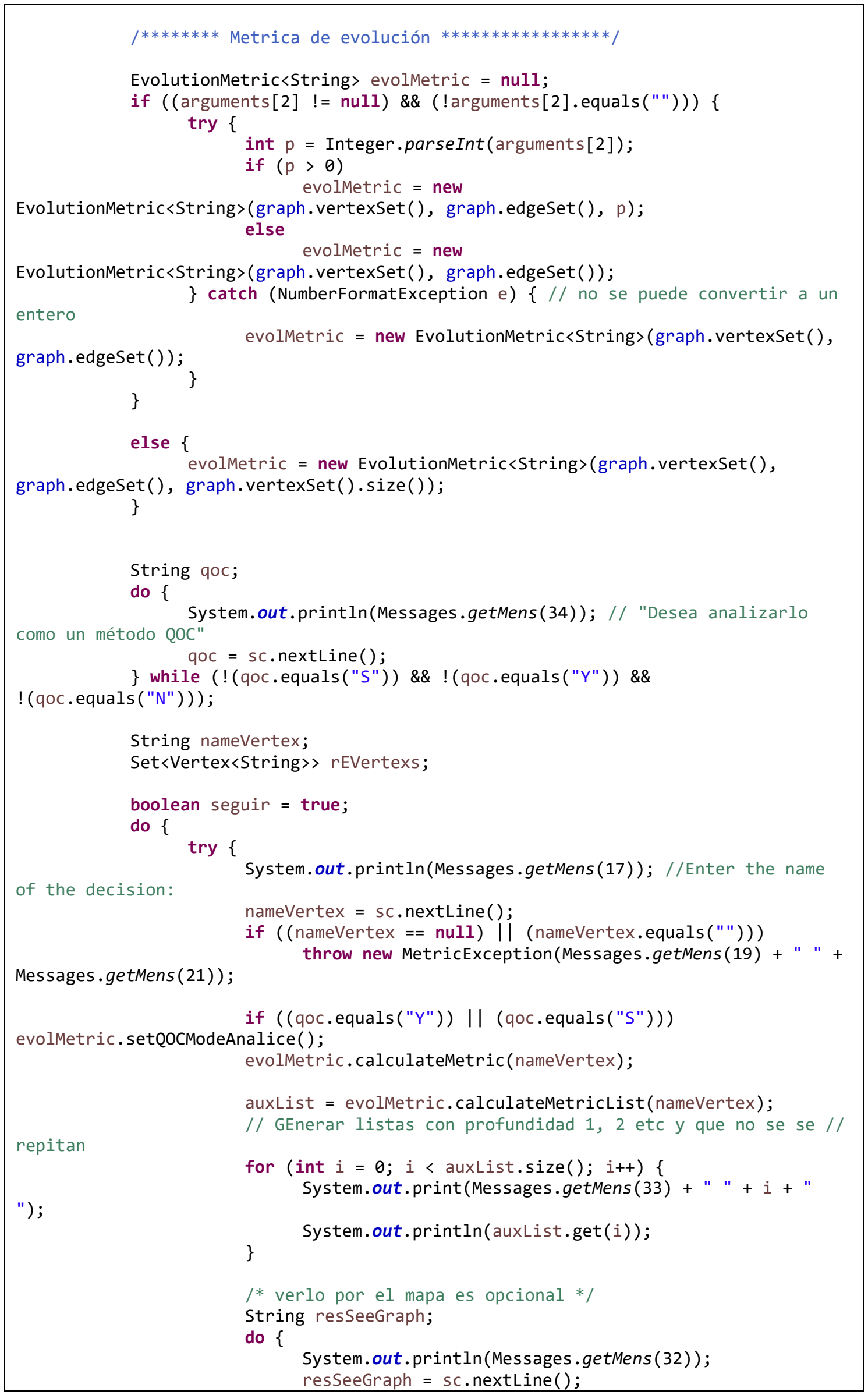




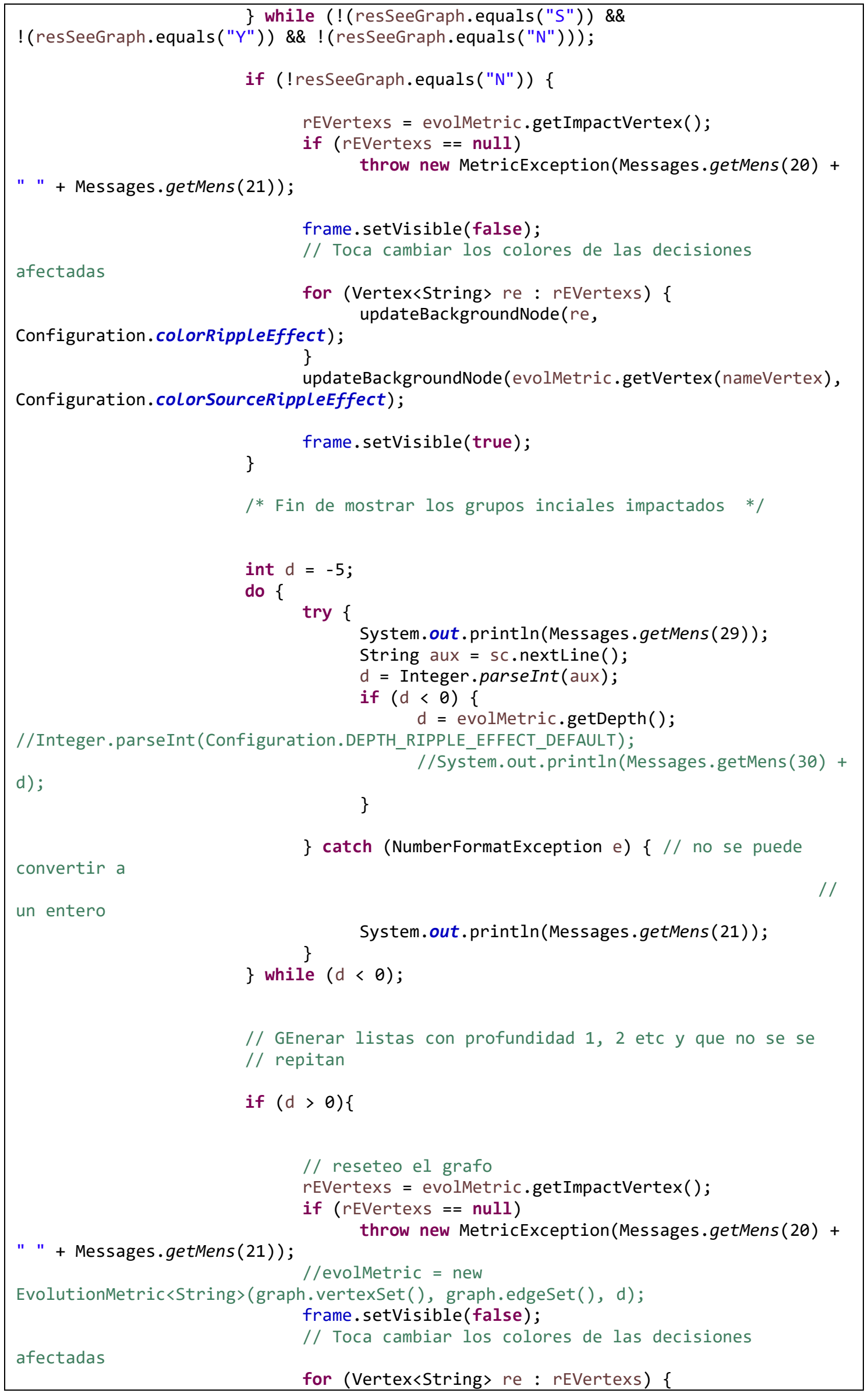




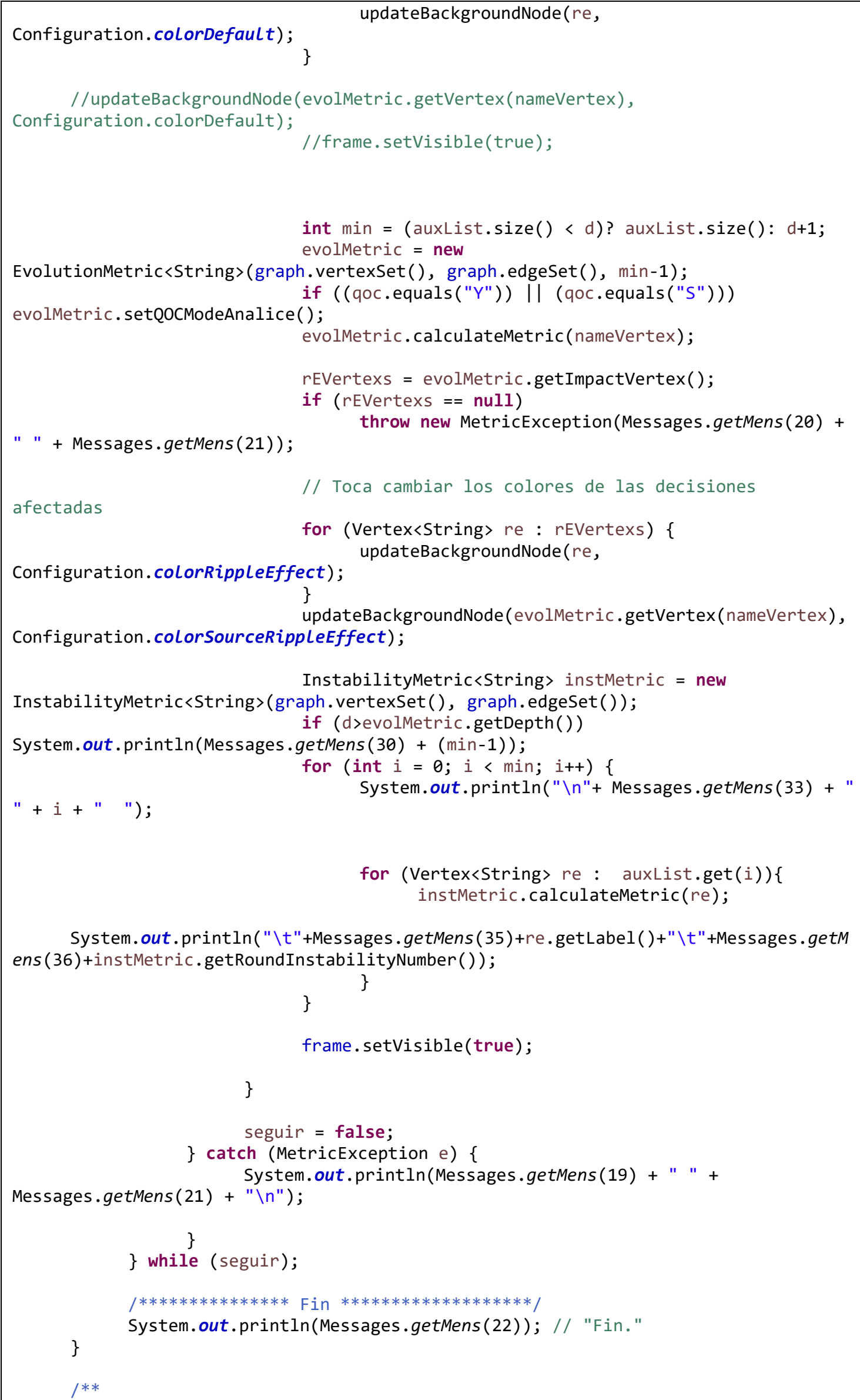

System.out.println("\t"+Messages.getMens(35)+re.getLabel( )+" \t"+Messages.getM ens(36)+instMetric.getRoundInstabilityNumber( )); 


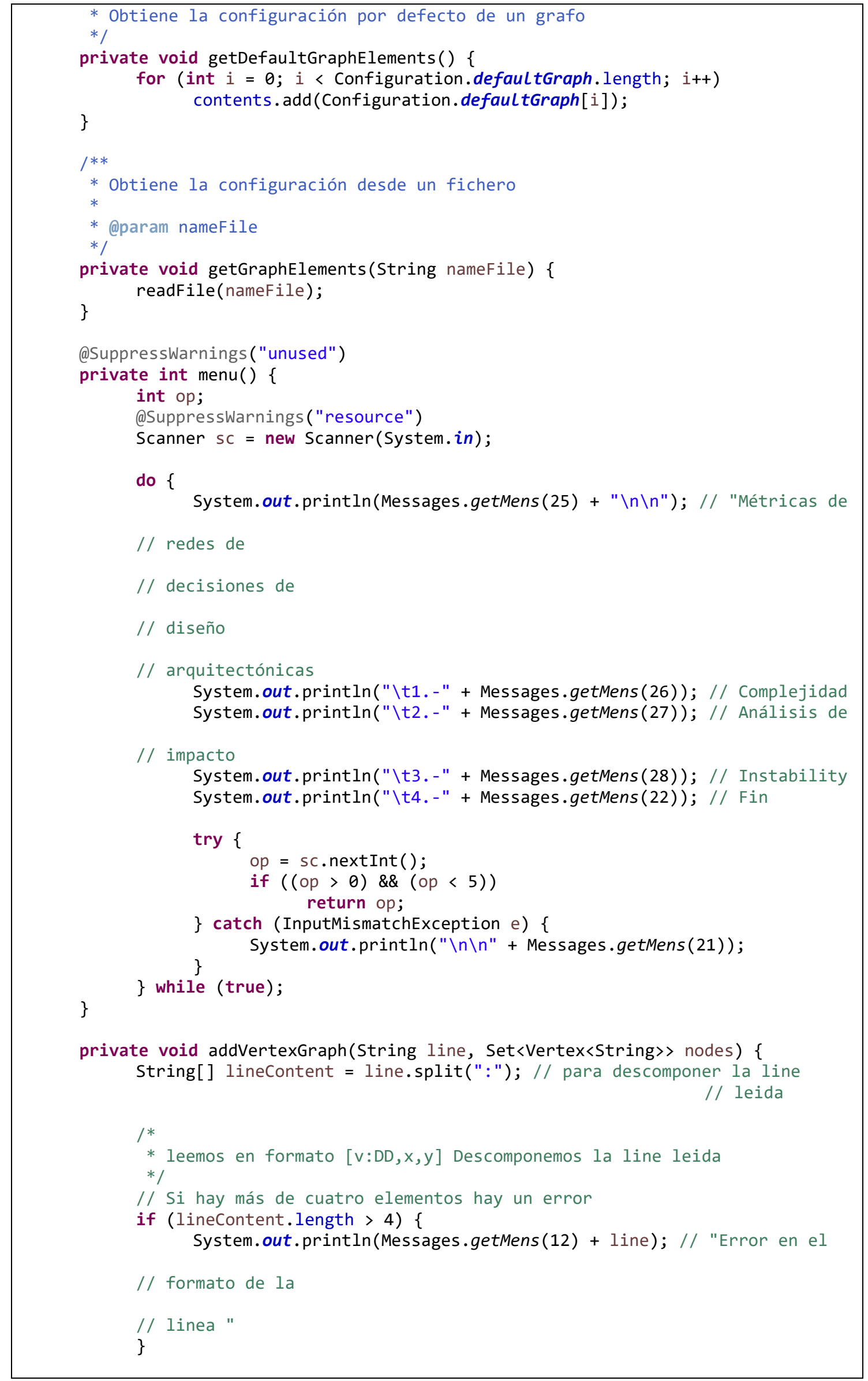




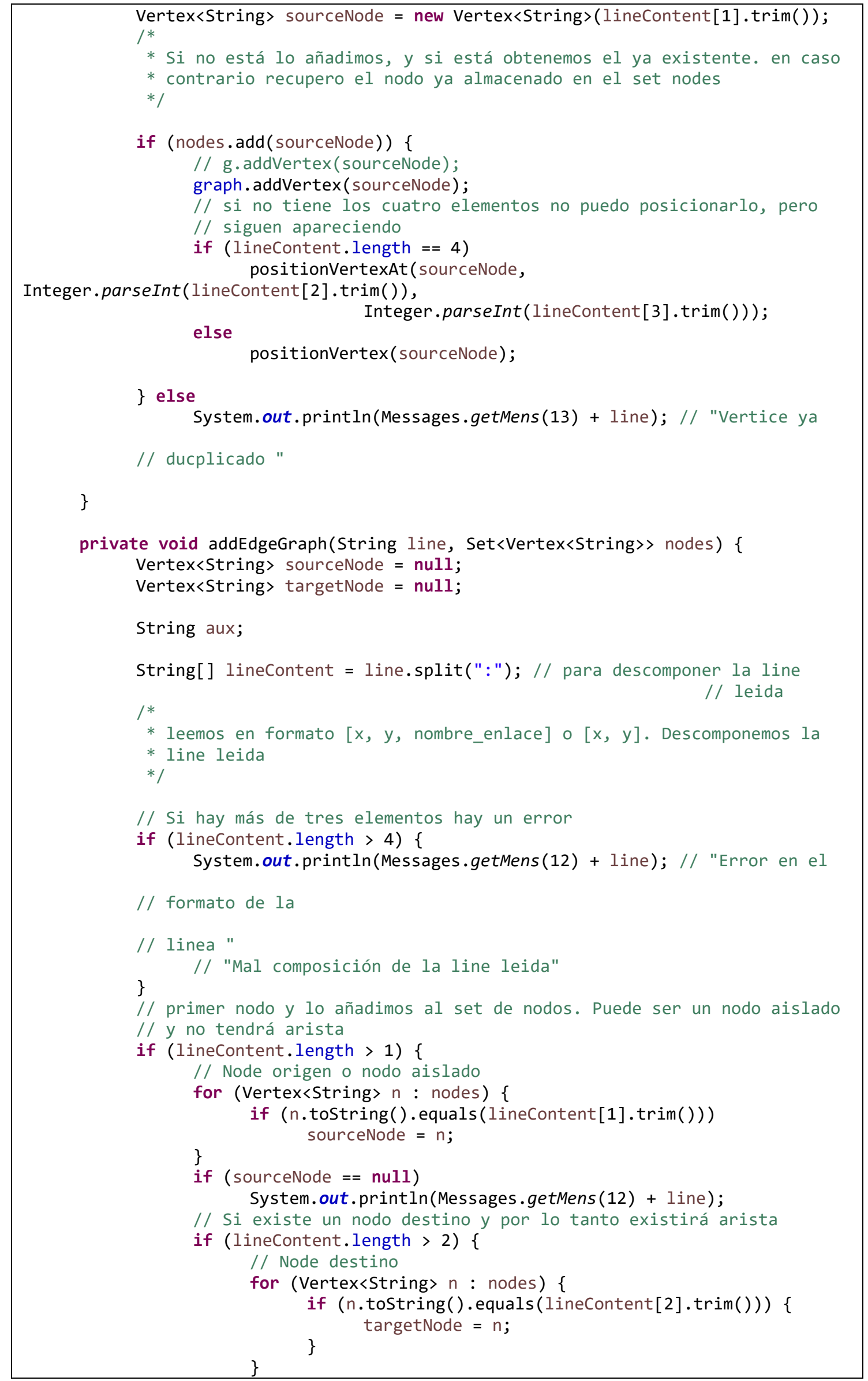




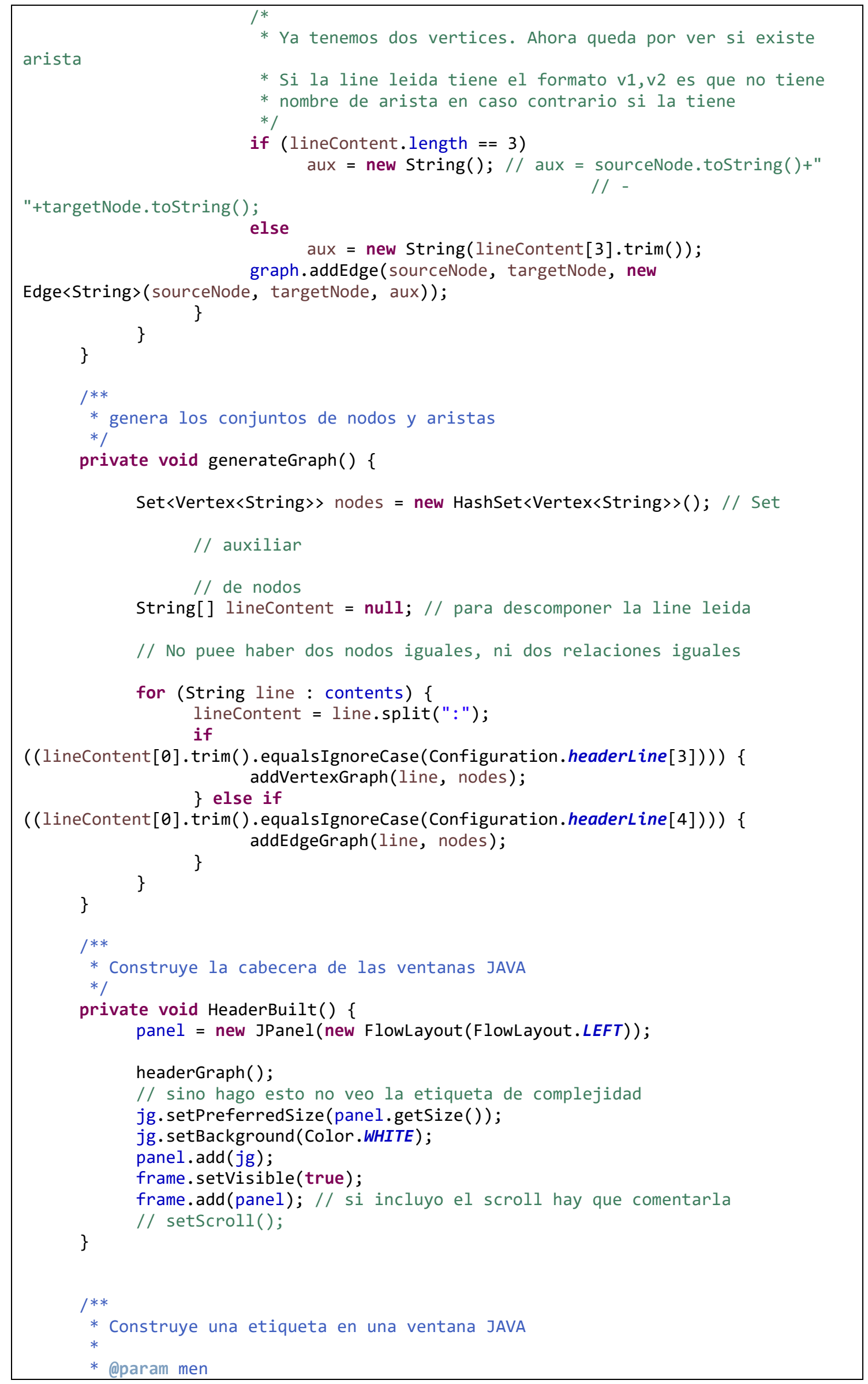




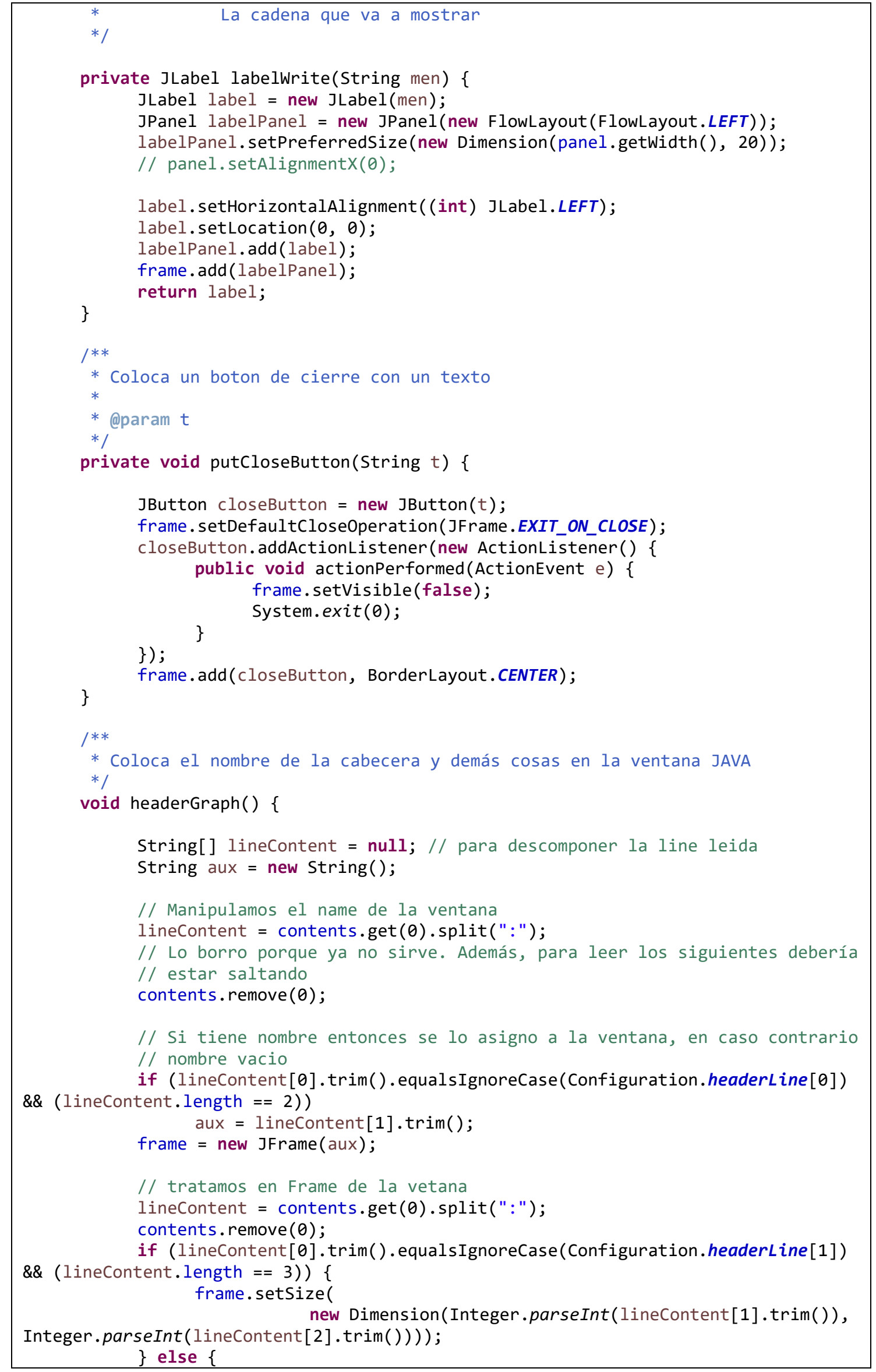




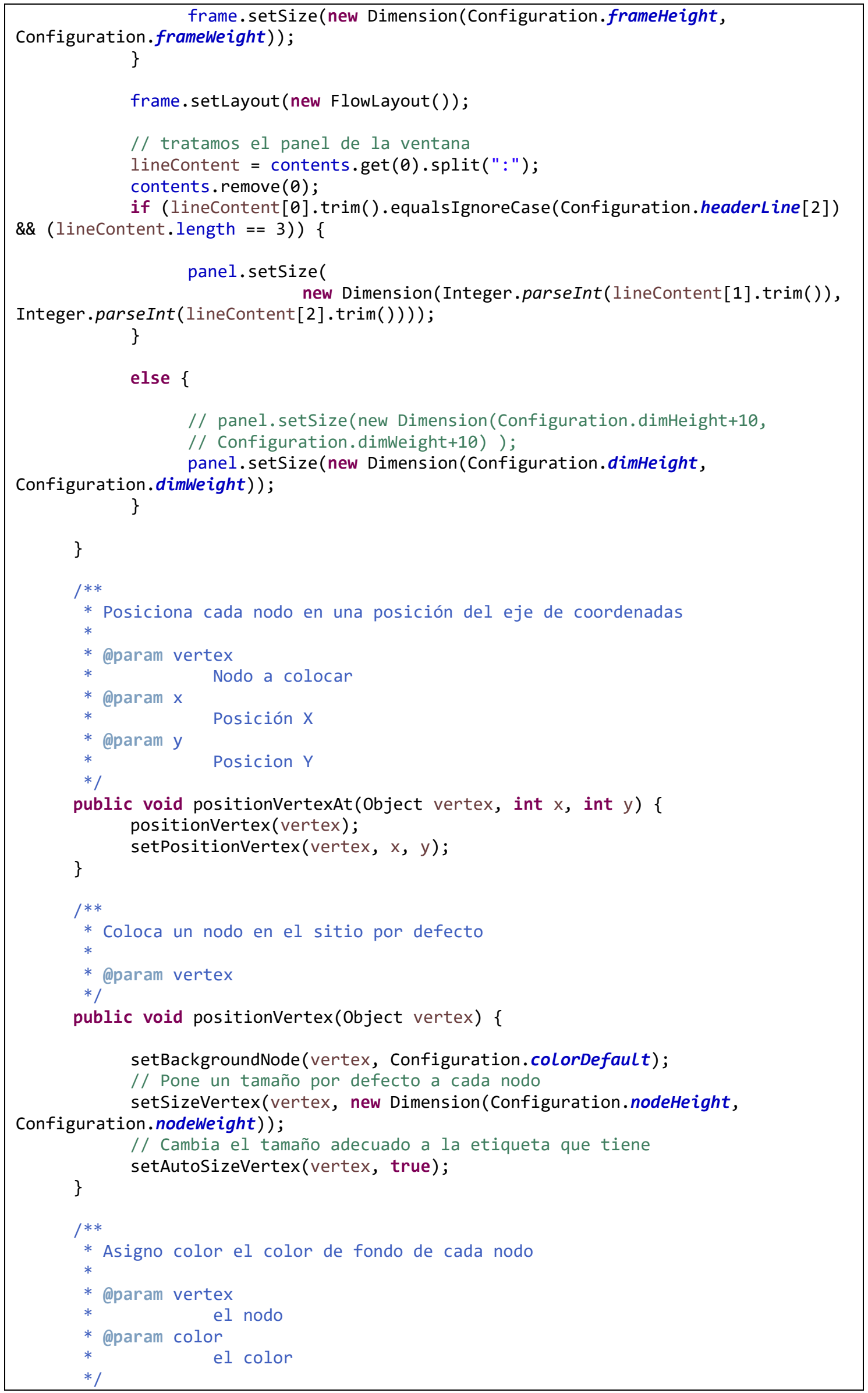




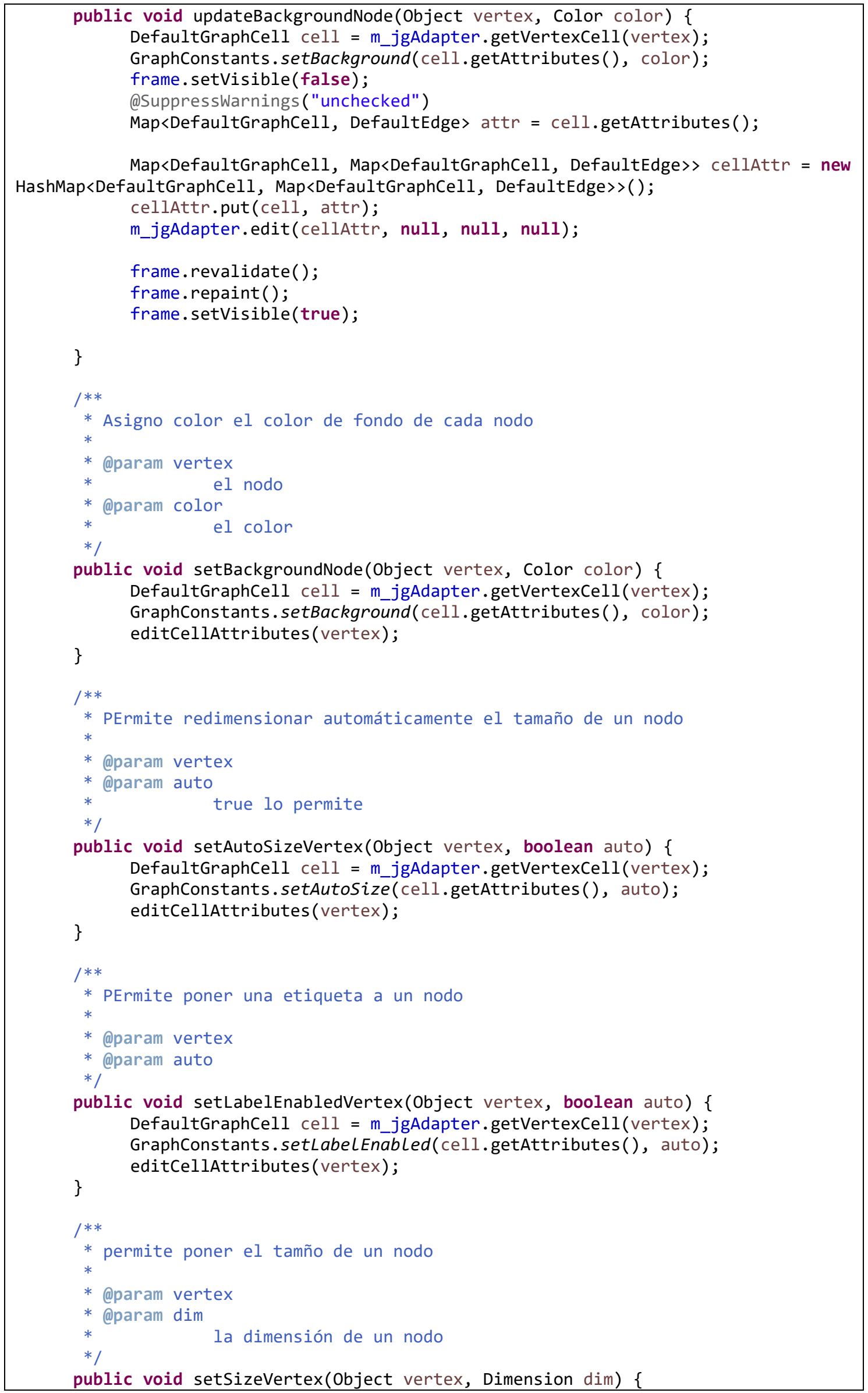




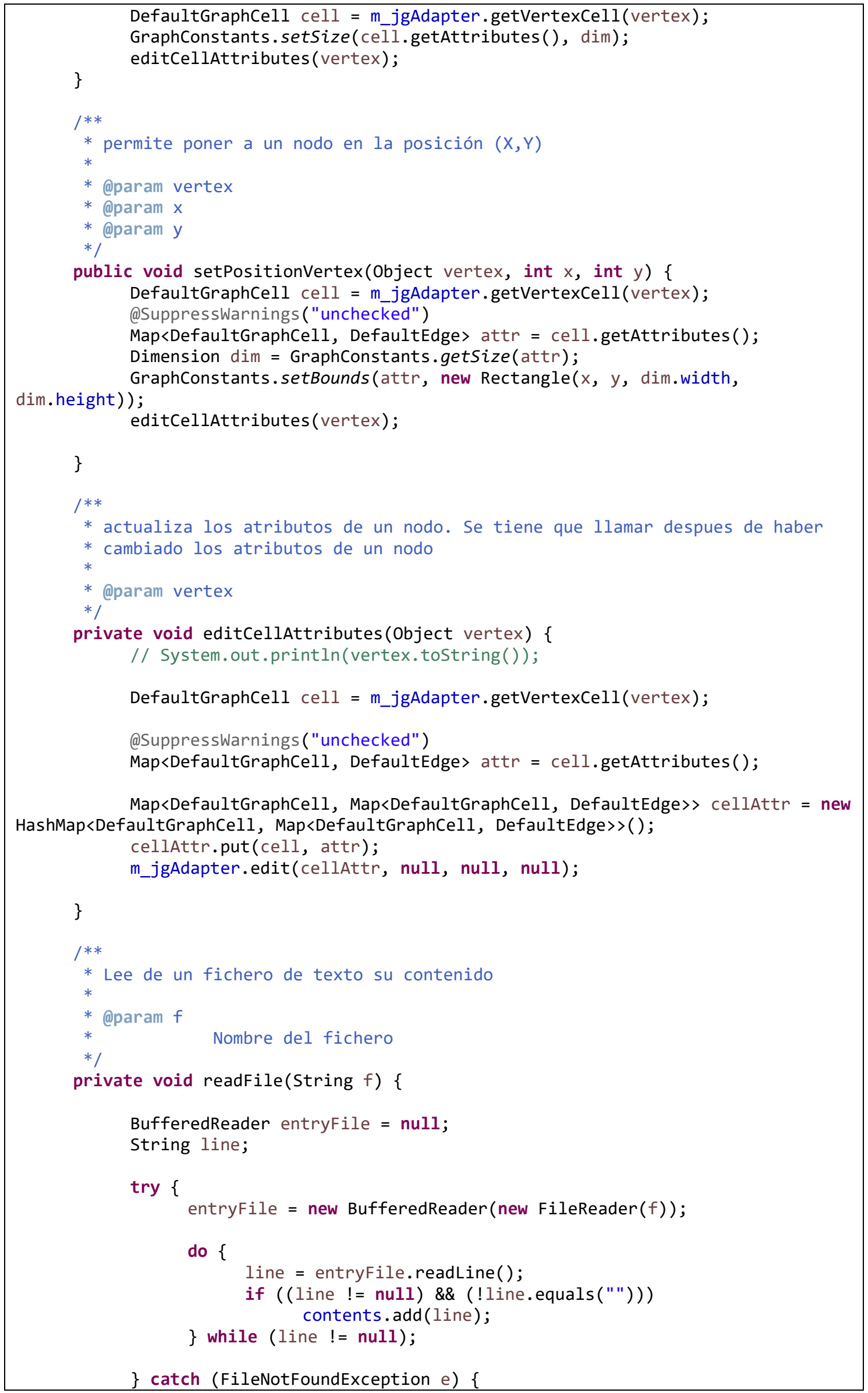




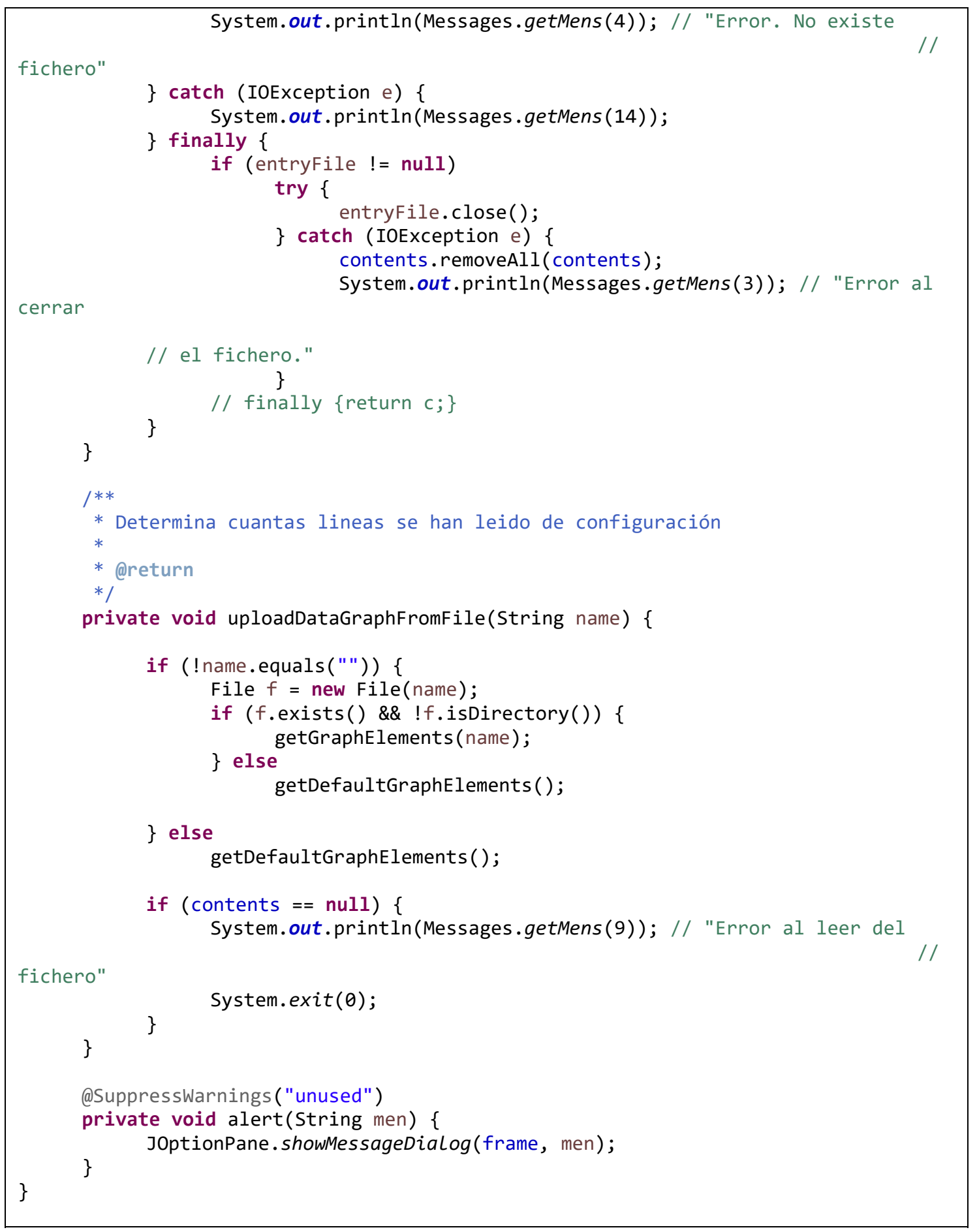

\section{Clase MetricsCalculator $<T>$.}

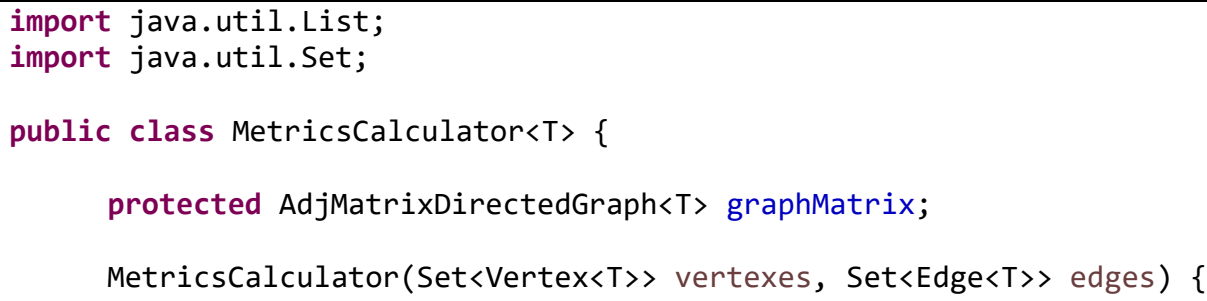




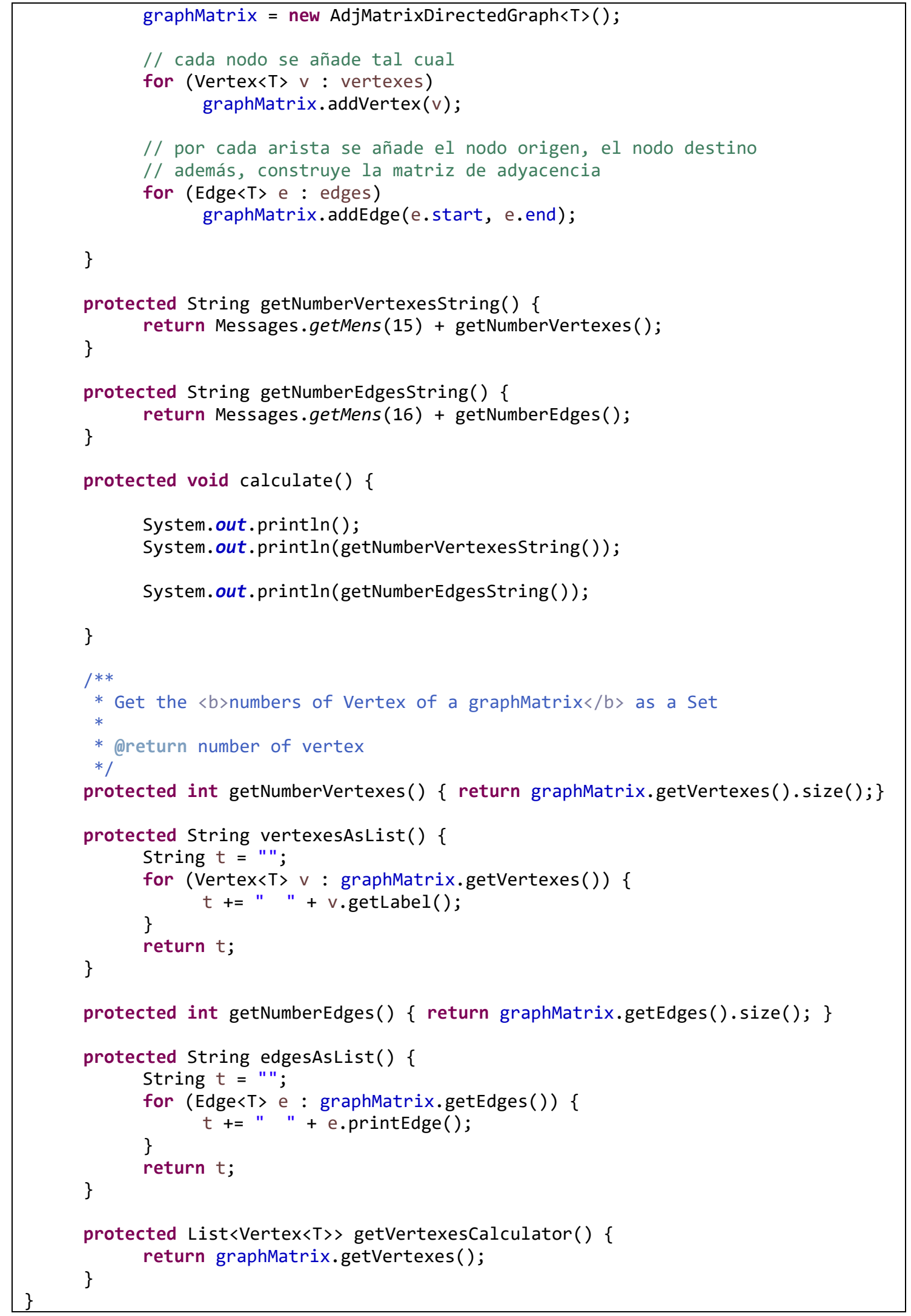




\section{Clase ComplexityMetric $<\mathrm{T}>$.}

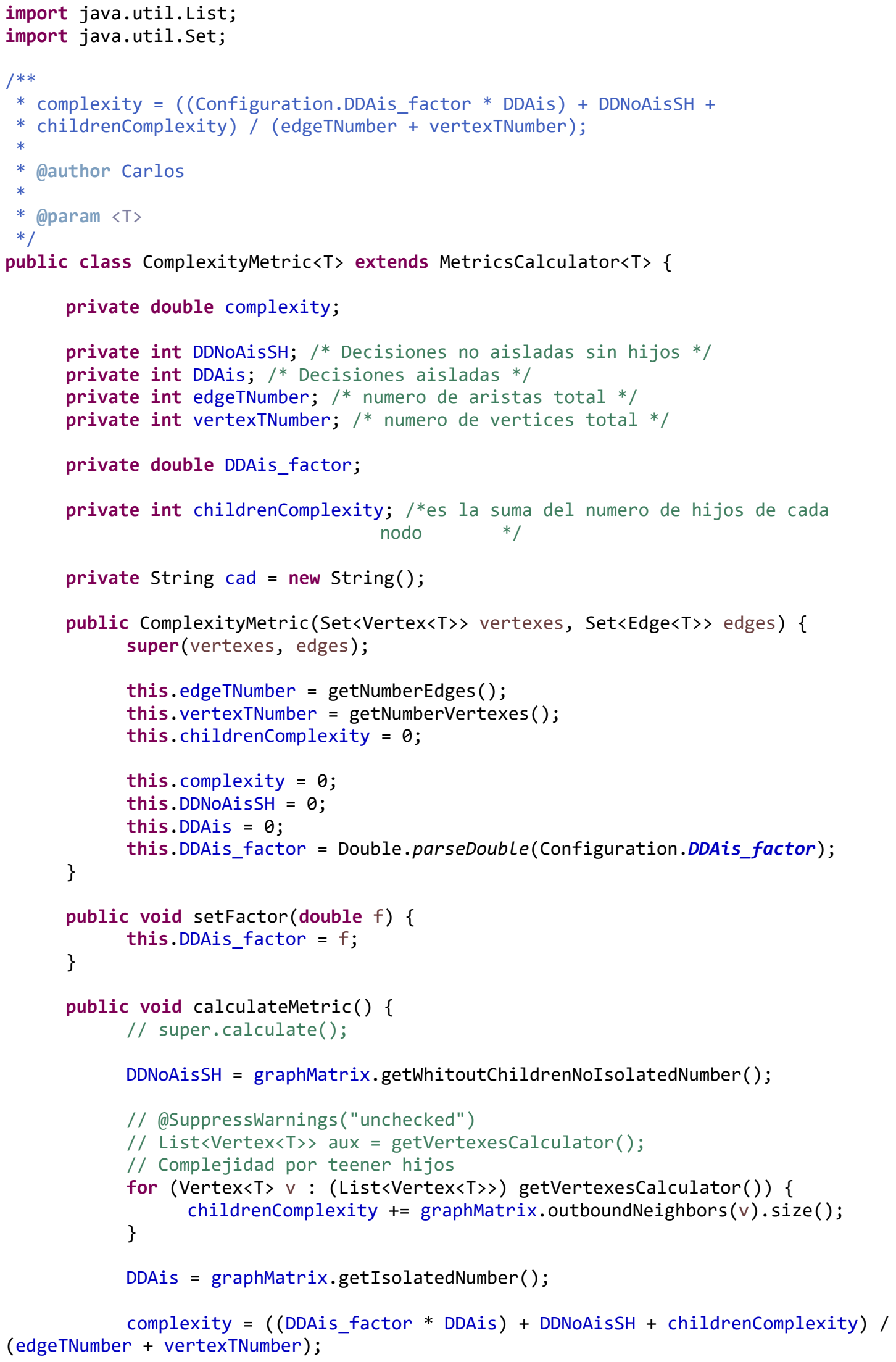




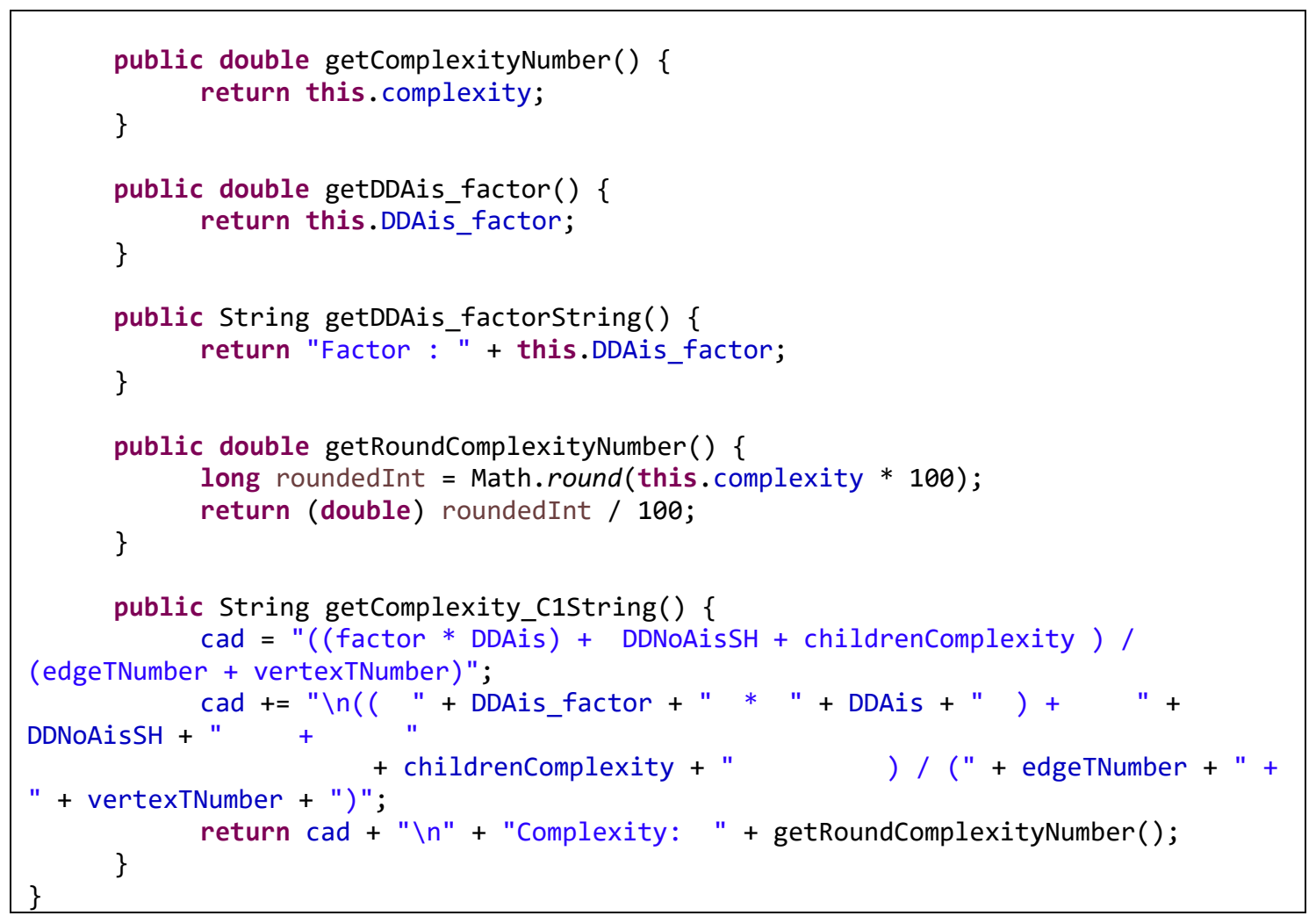

\section{Clase EvolutionMetric $<\mathrm{T}>$.}

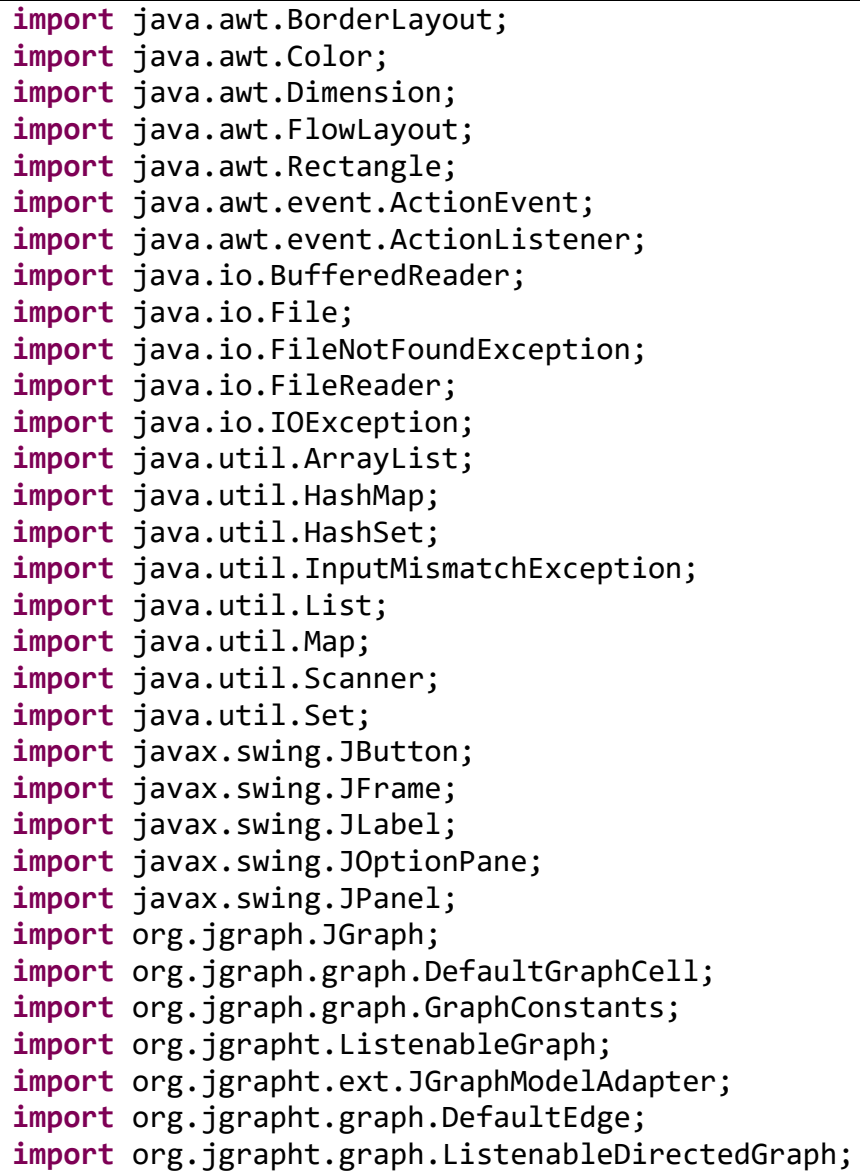




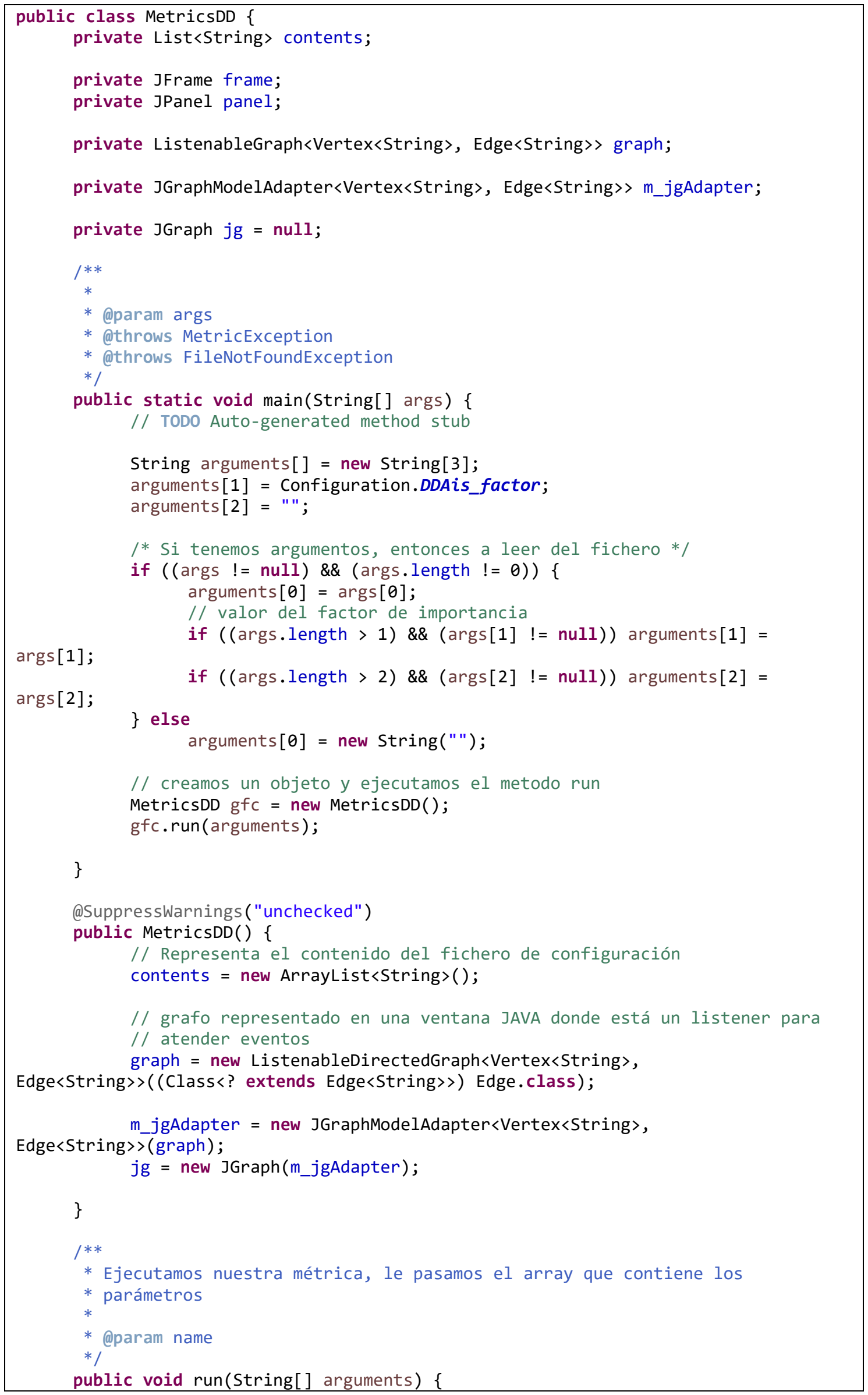




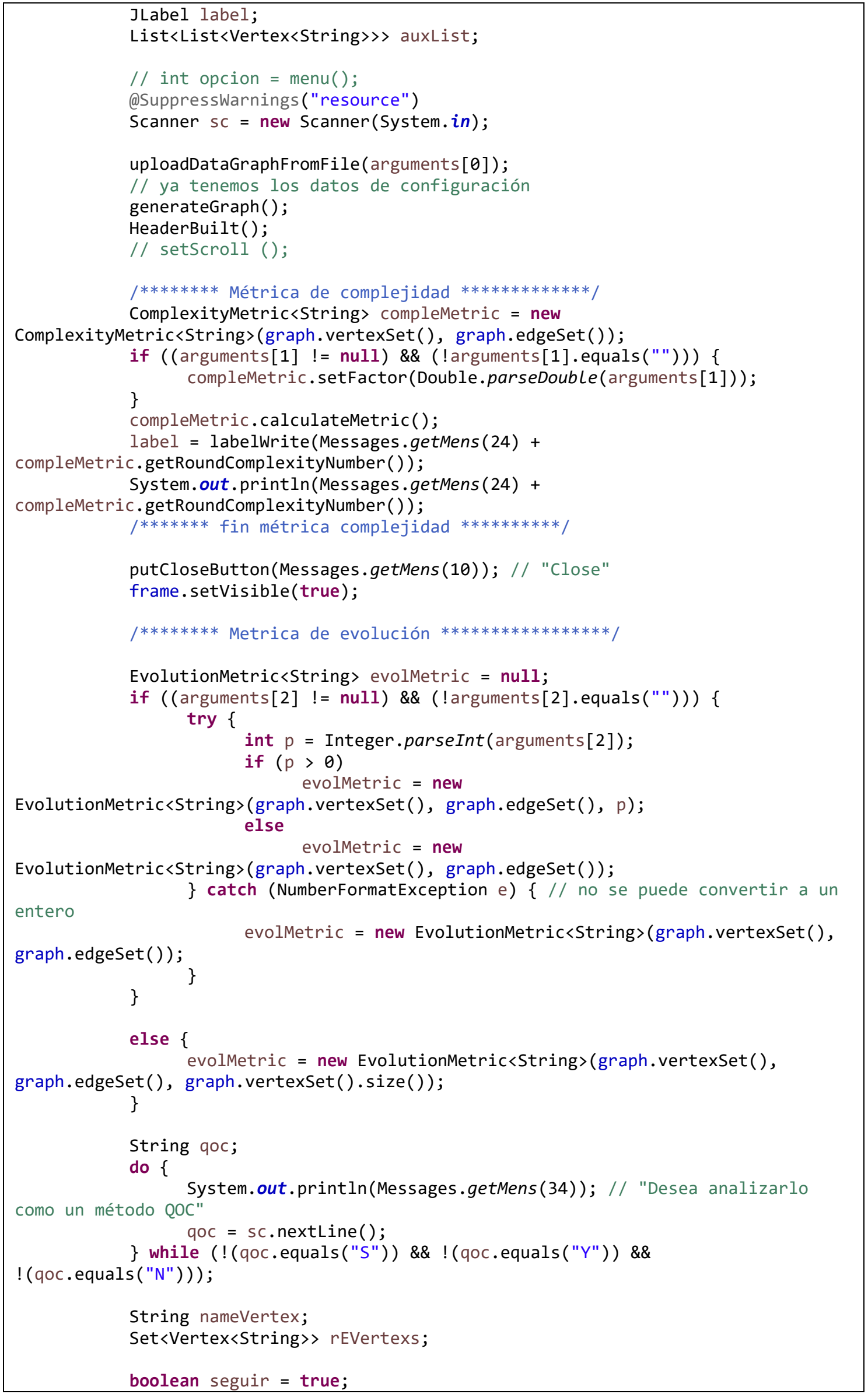




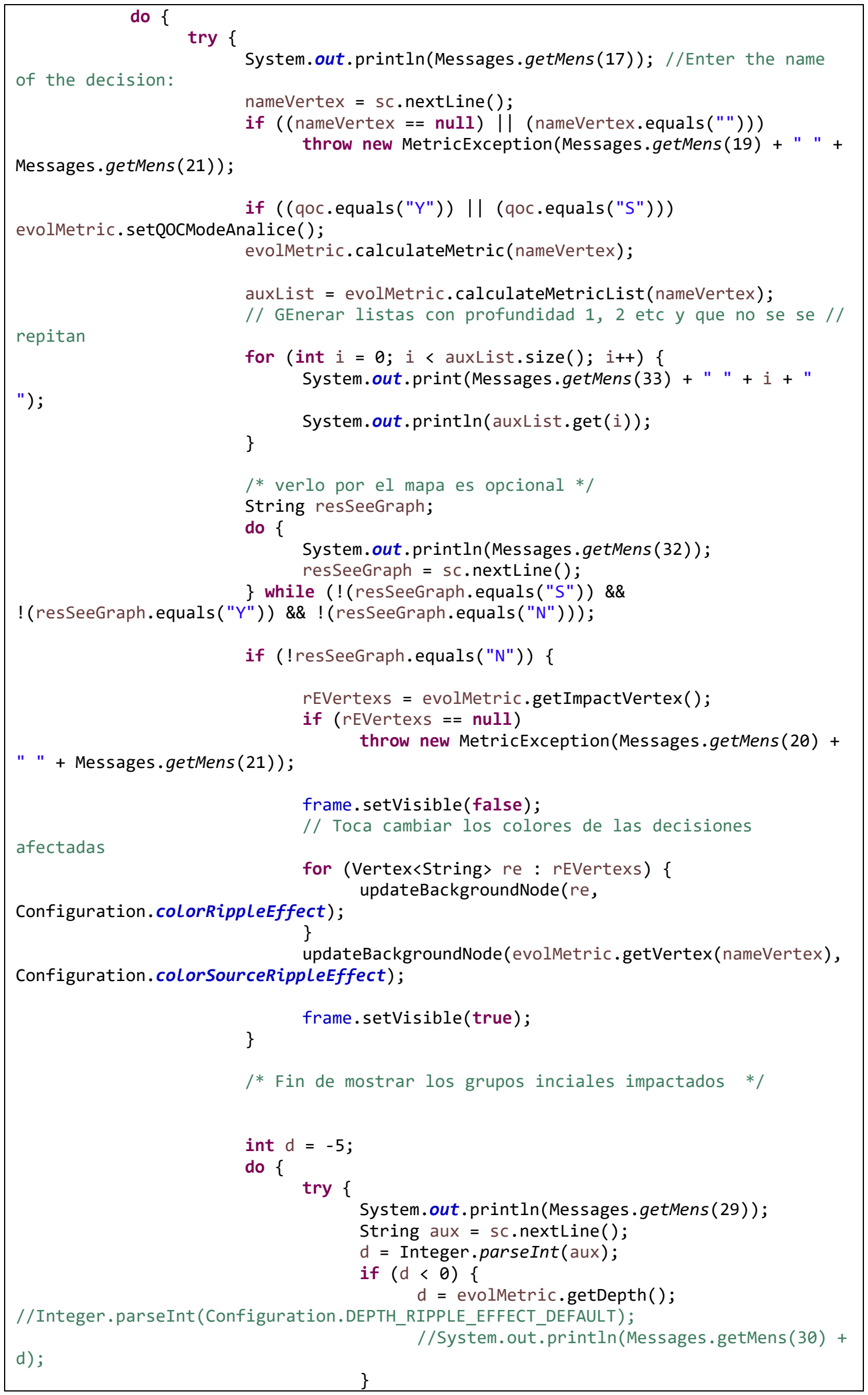




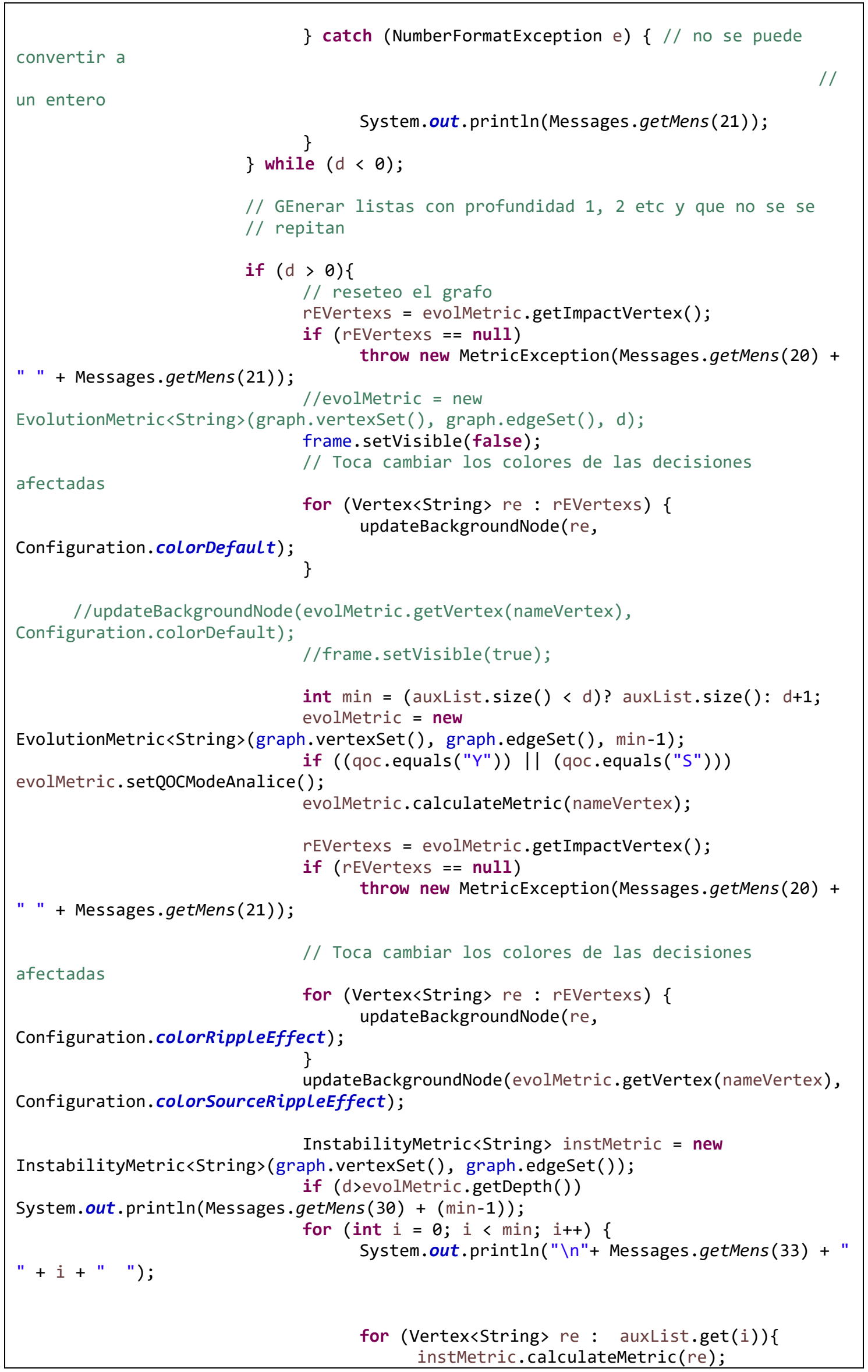




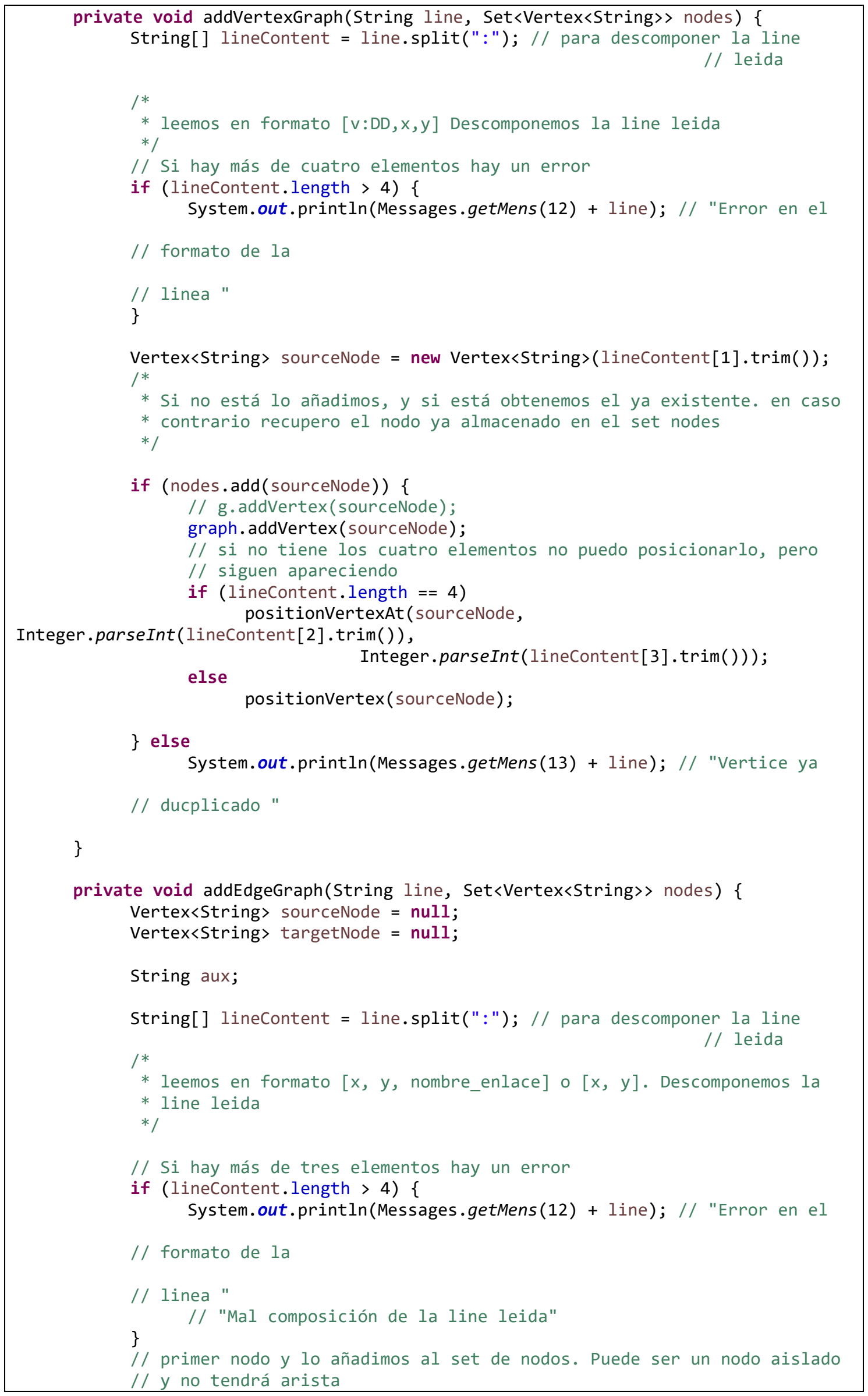




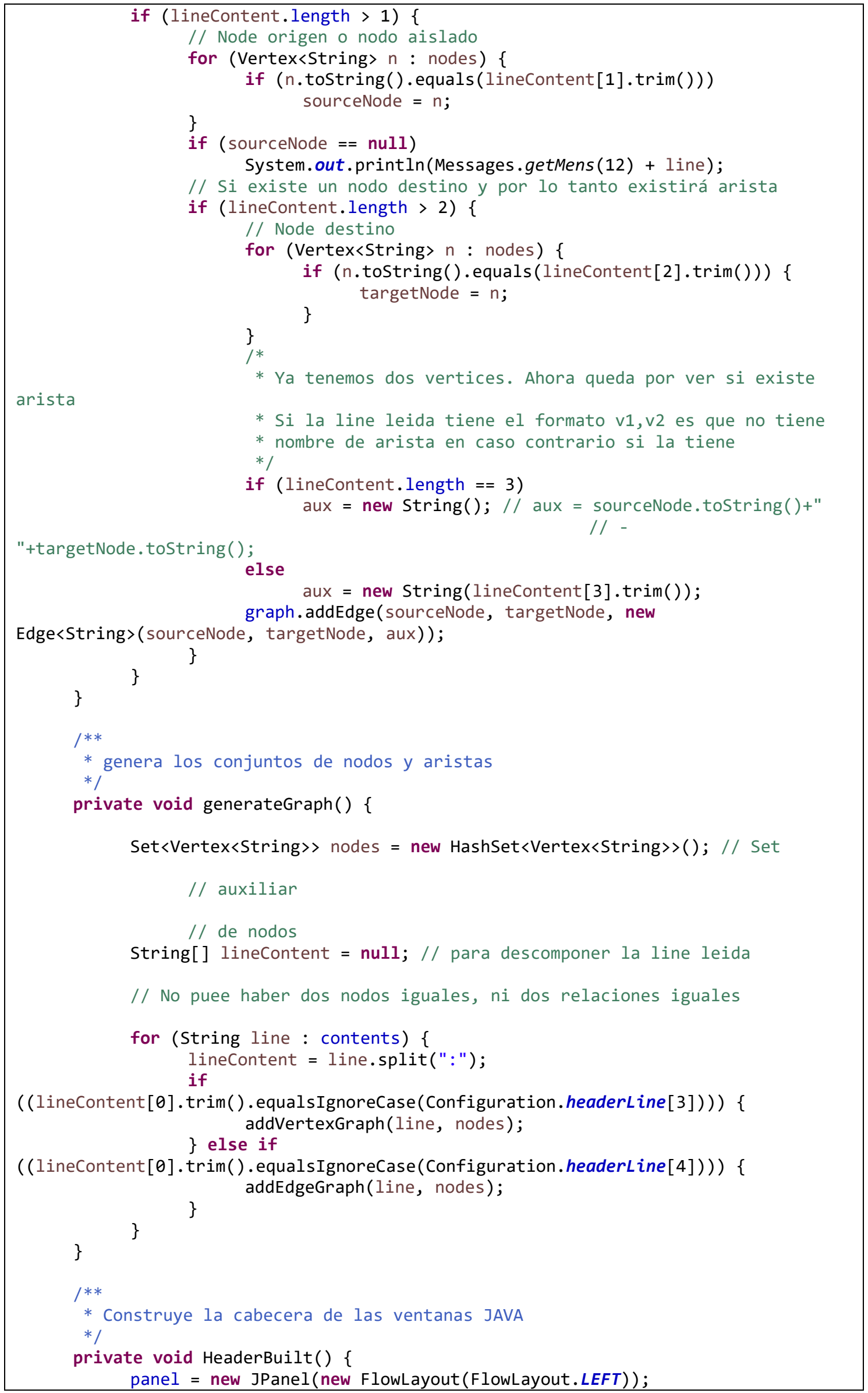




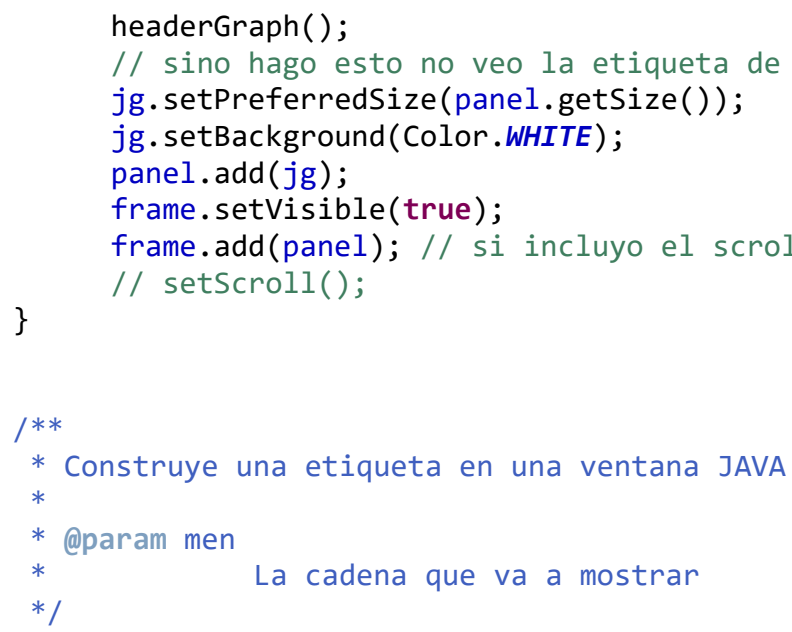




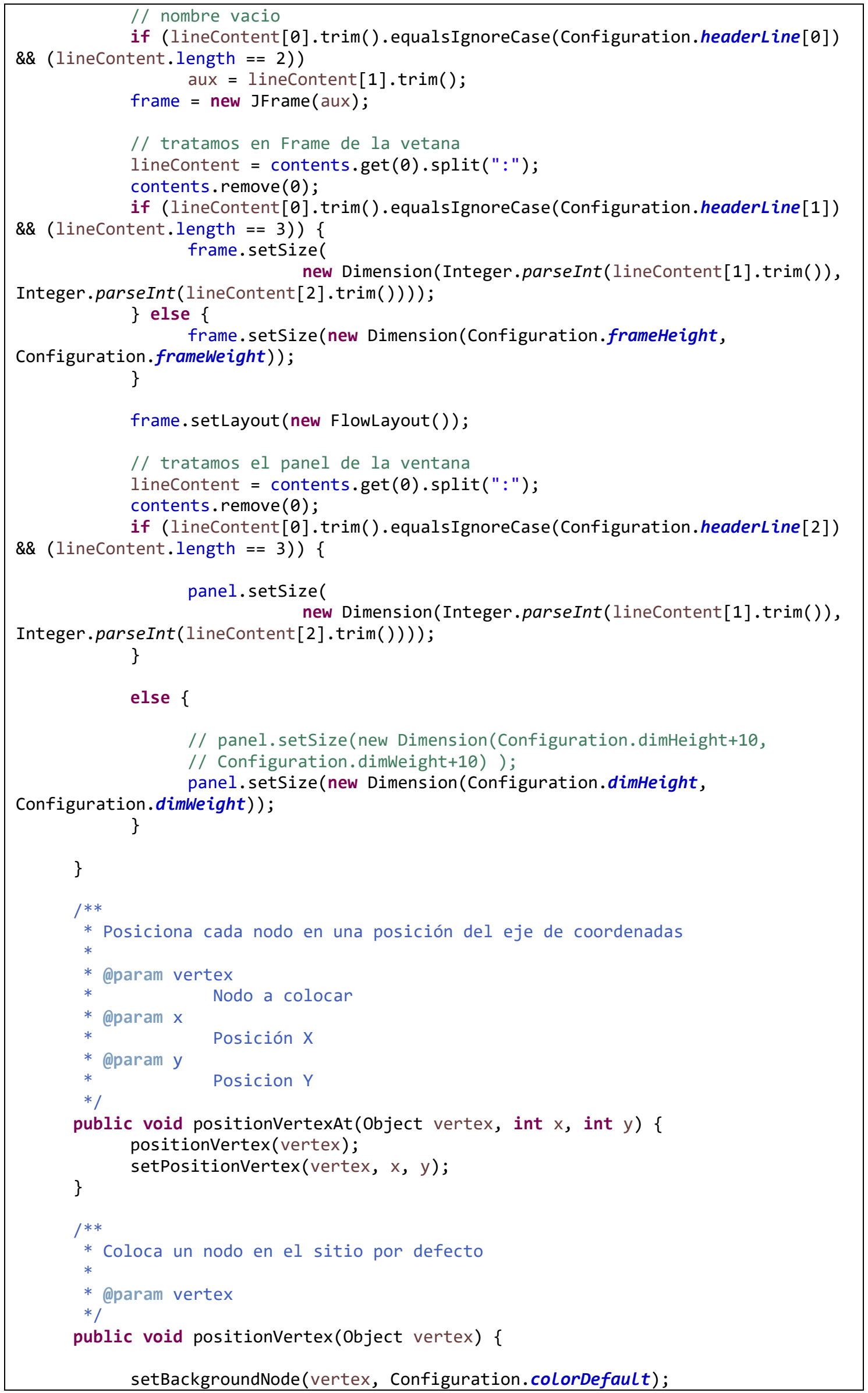




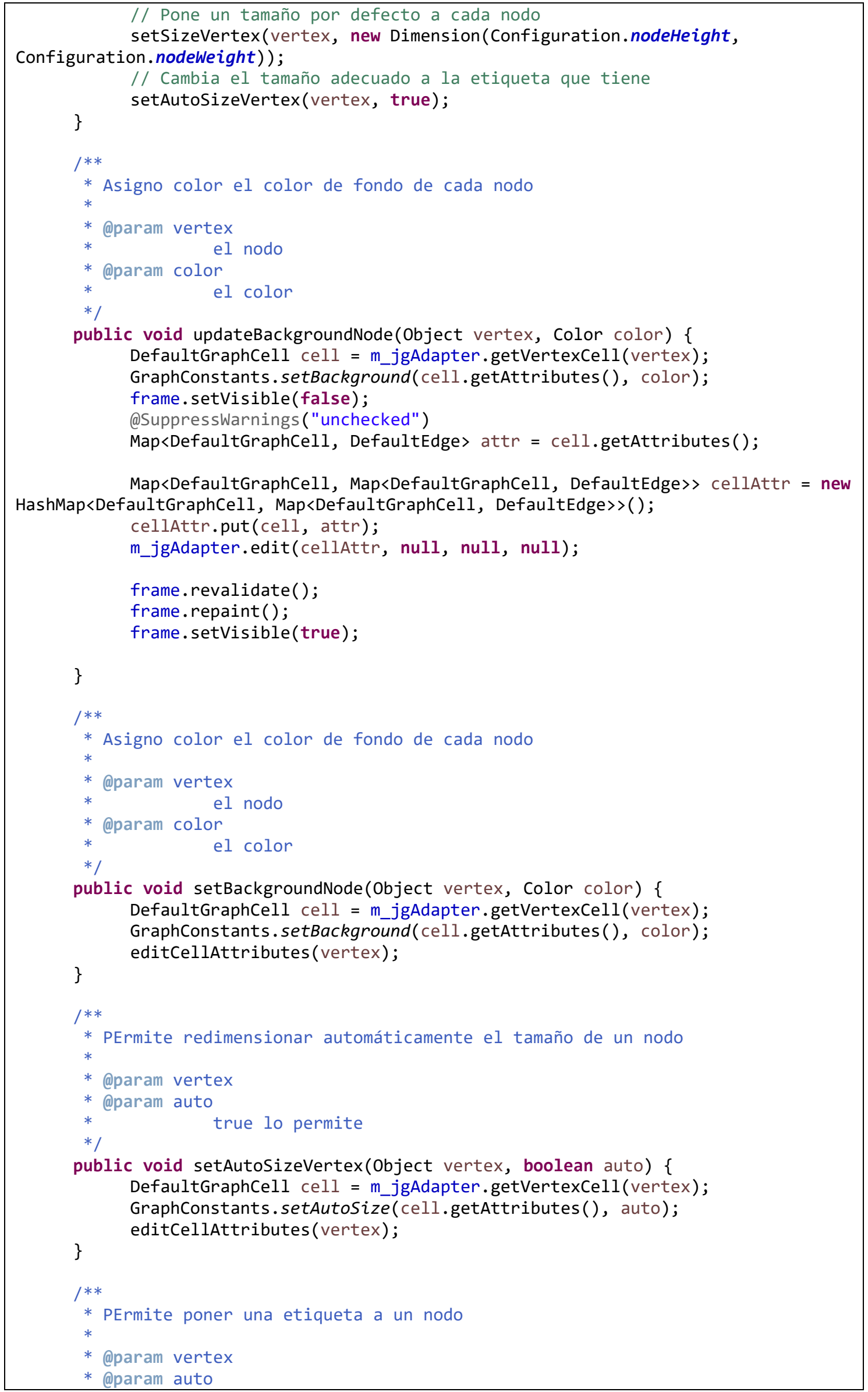




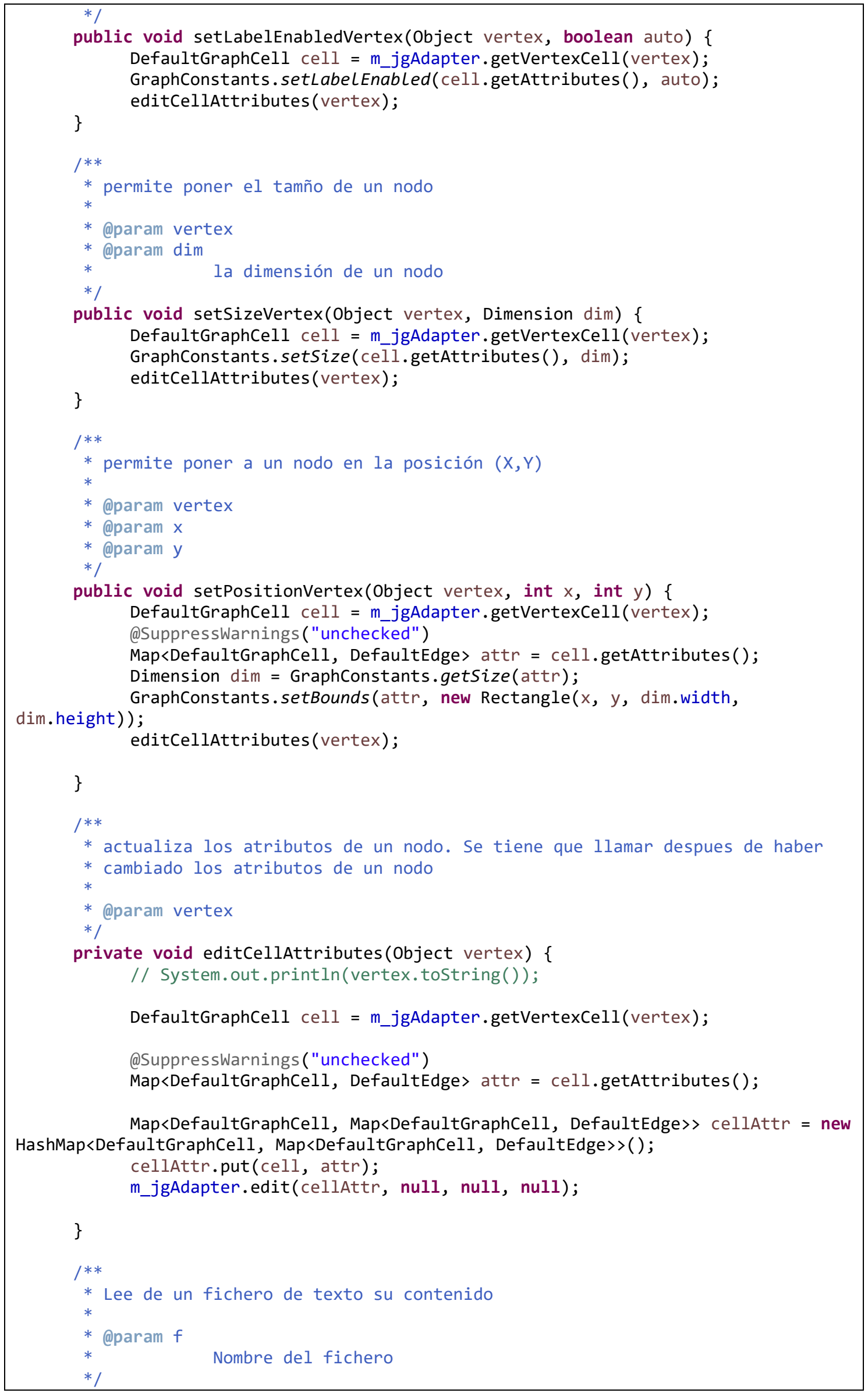




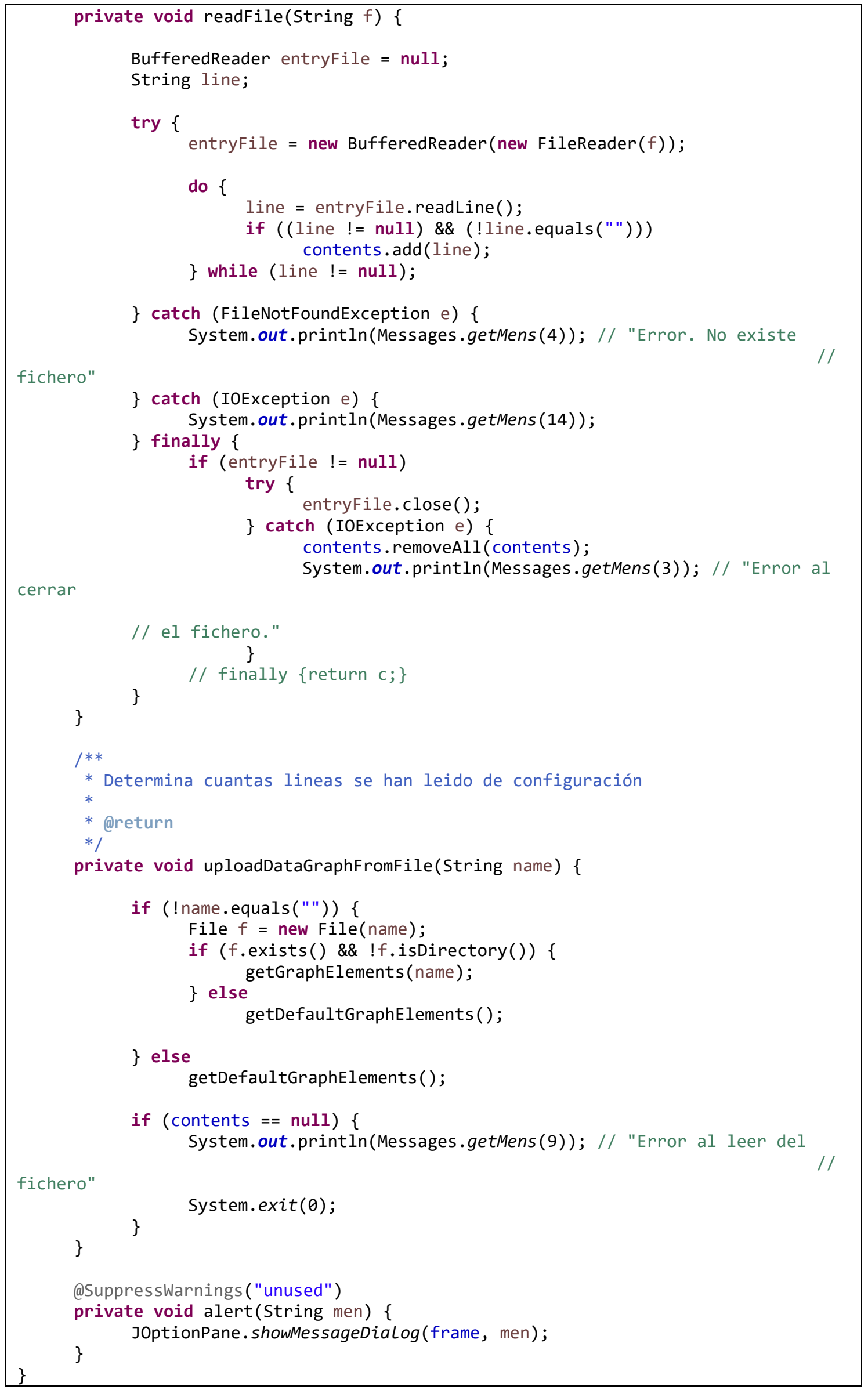




\section{Clase InstabilityMetric $<\mathrm{T}>$.}

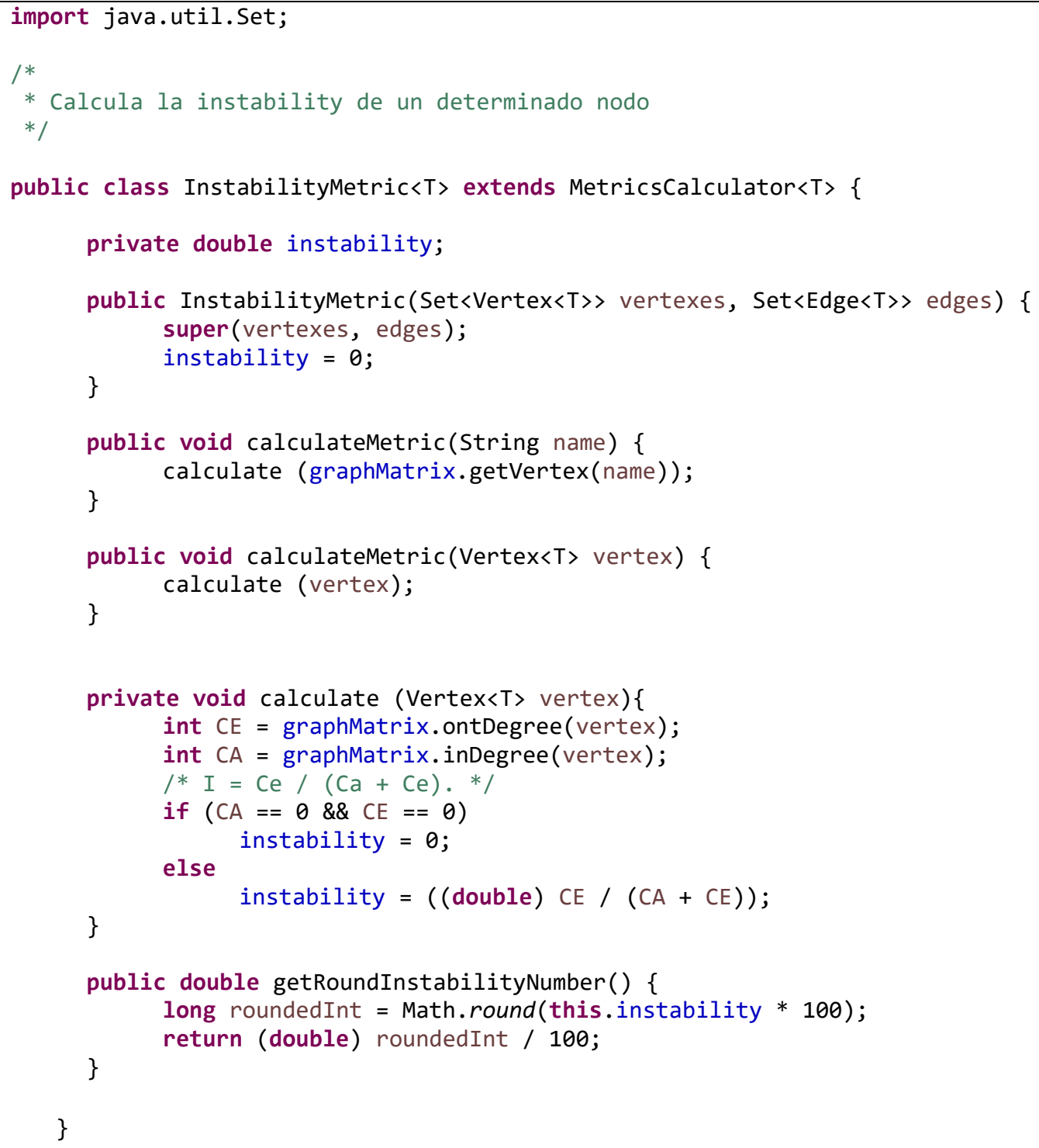

\section{Clase RippleEffect $<T>$.}

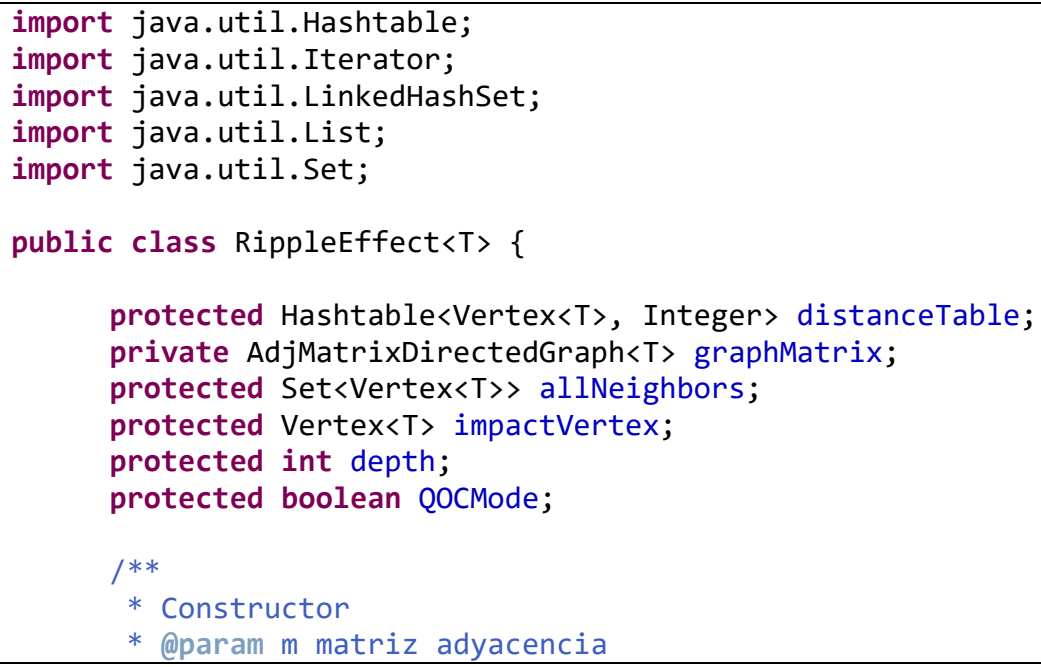




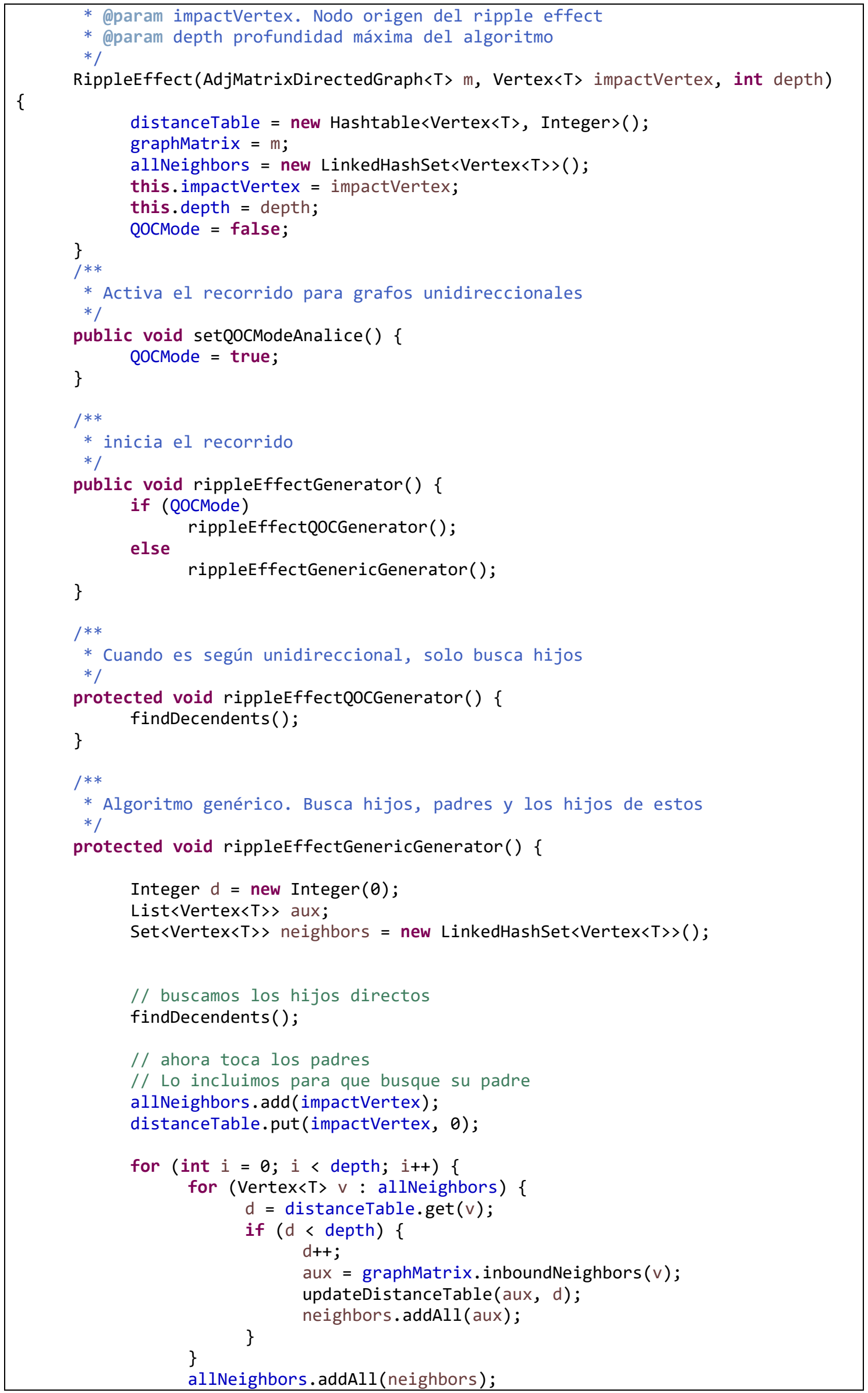




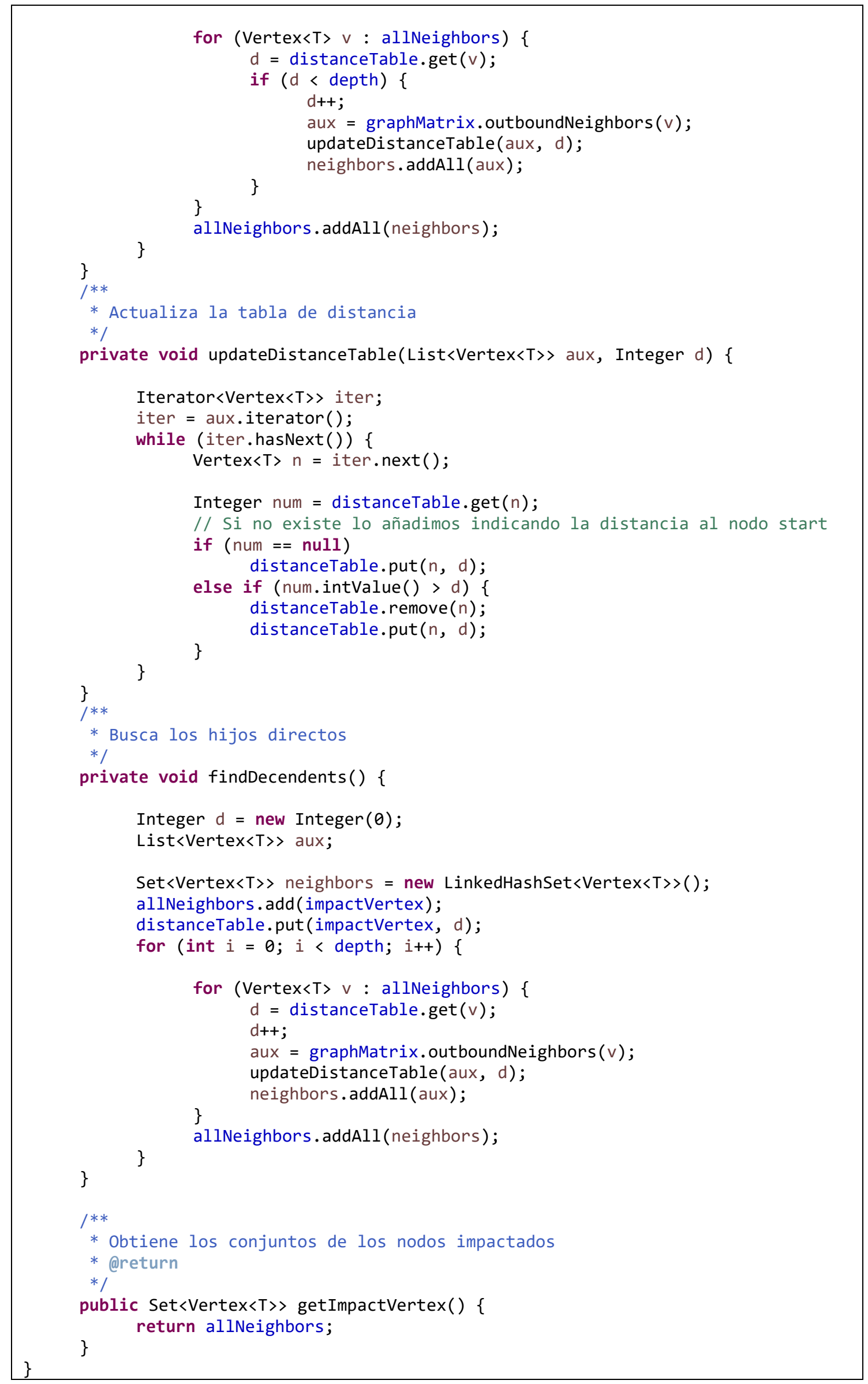




\section{Clase ImpactedDDByLevels $<T>$.}

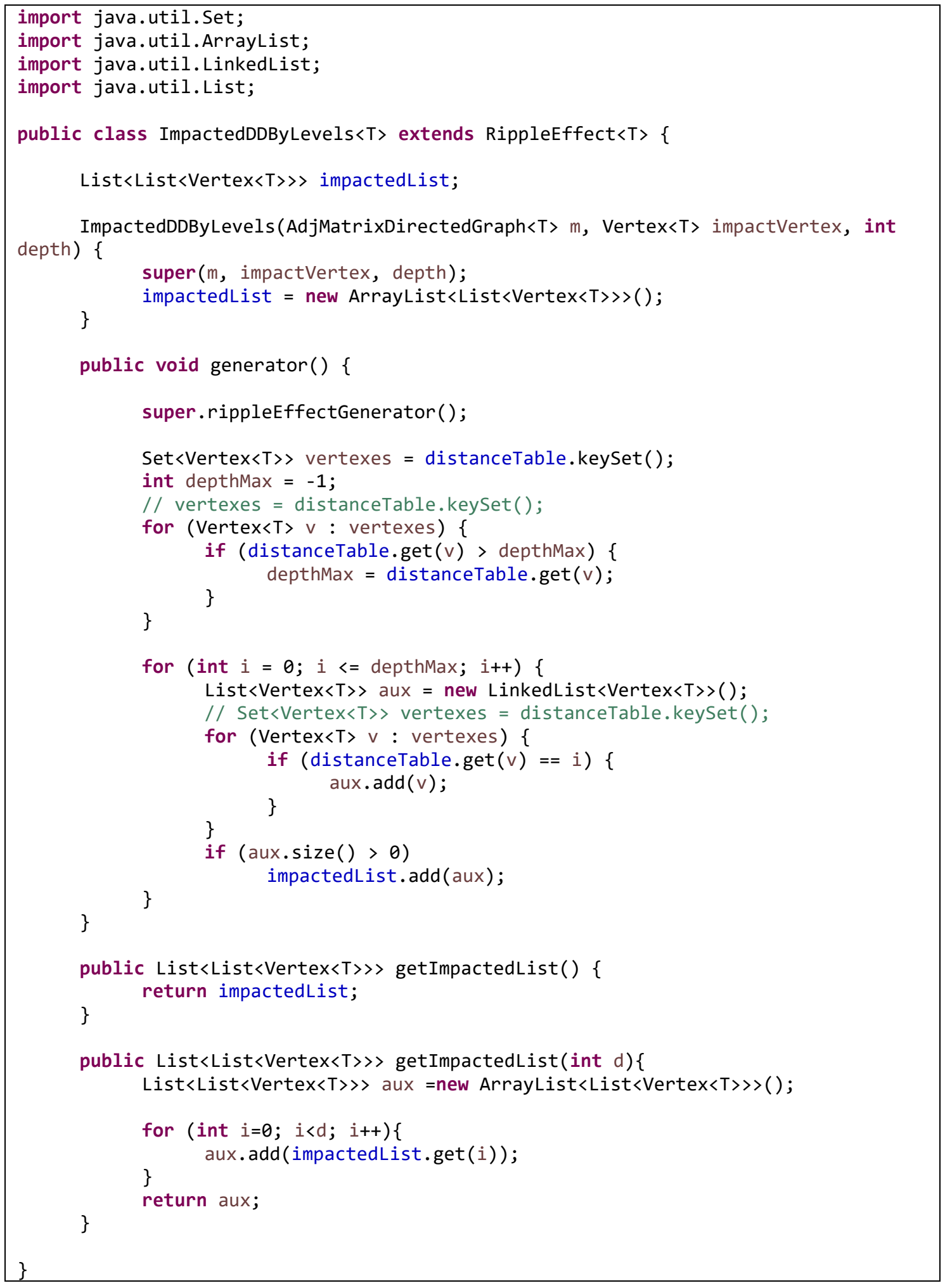




\section{Clase AdjMatrixDirectedGraph $<T>$.}

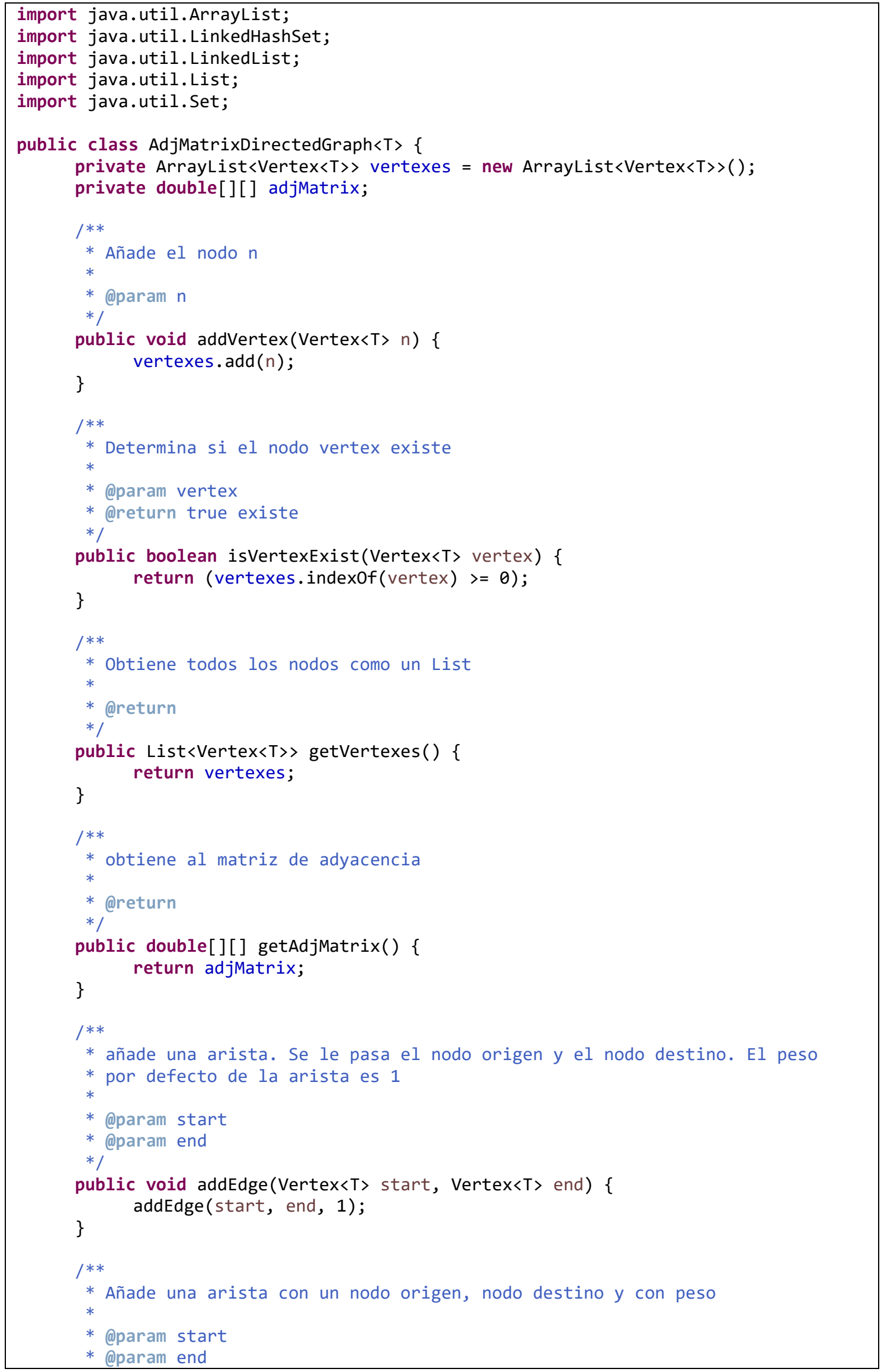




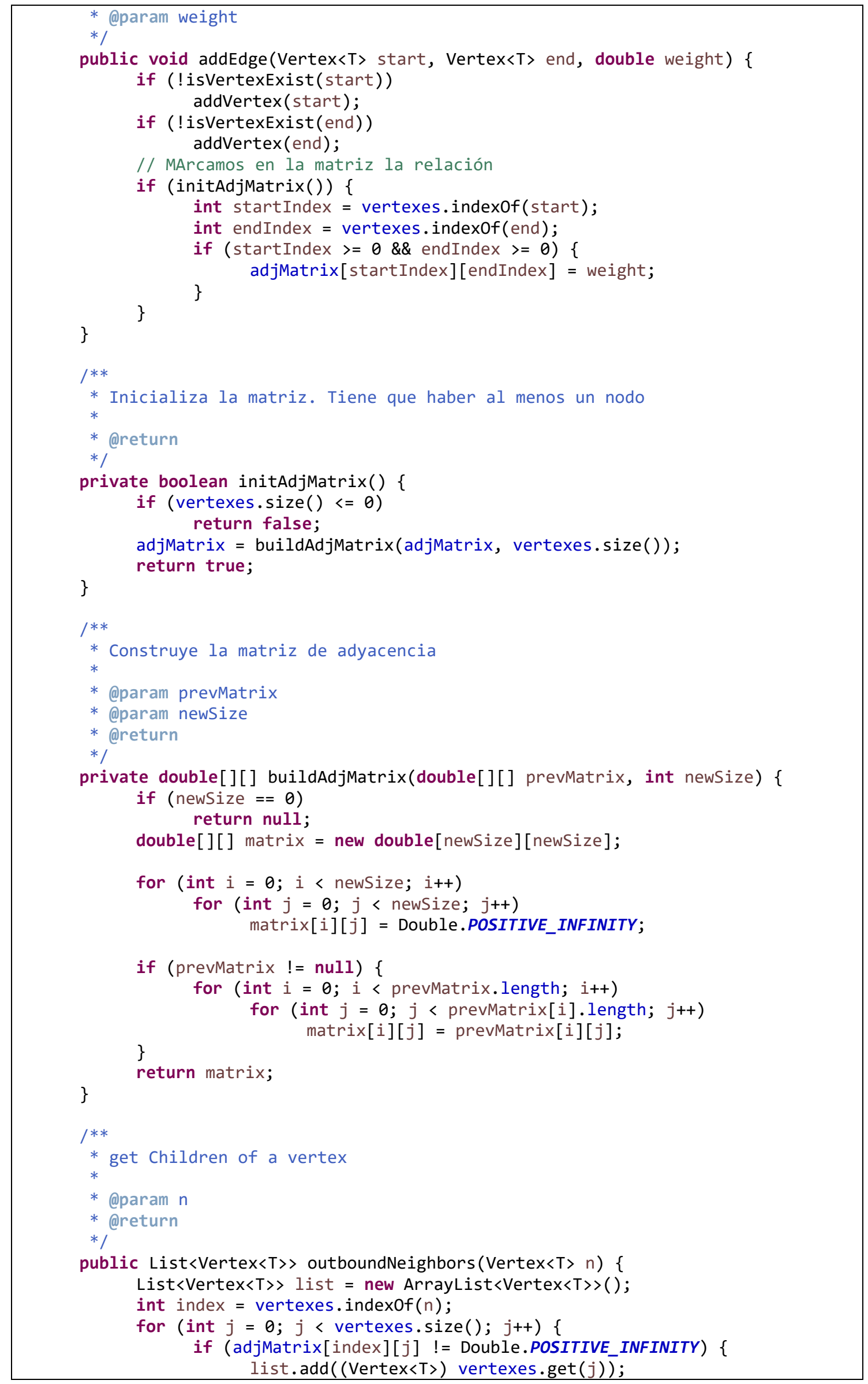




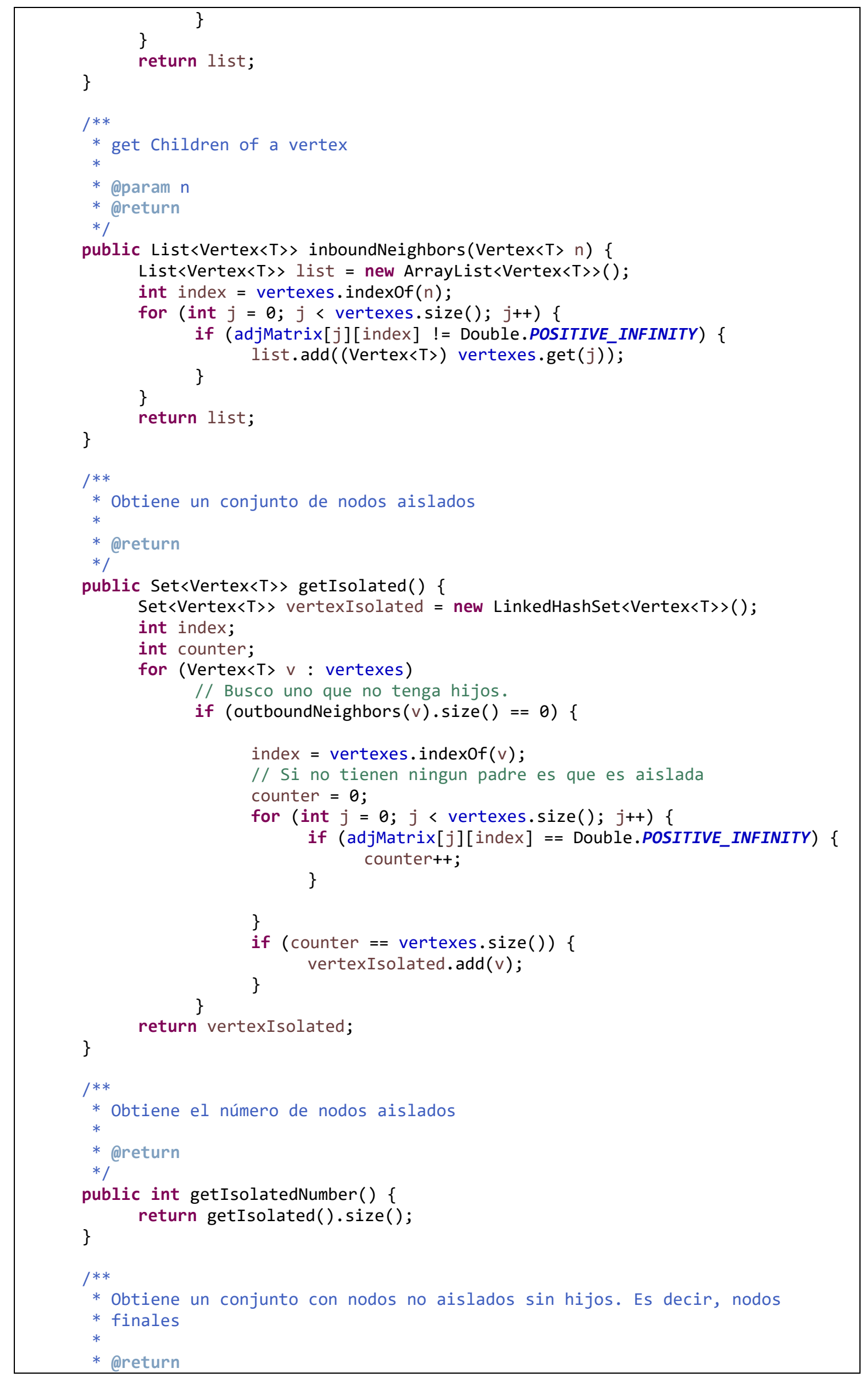




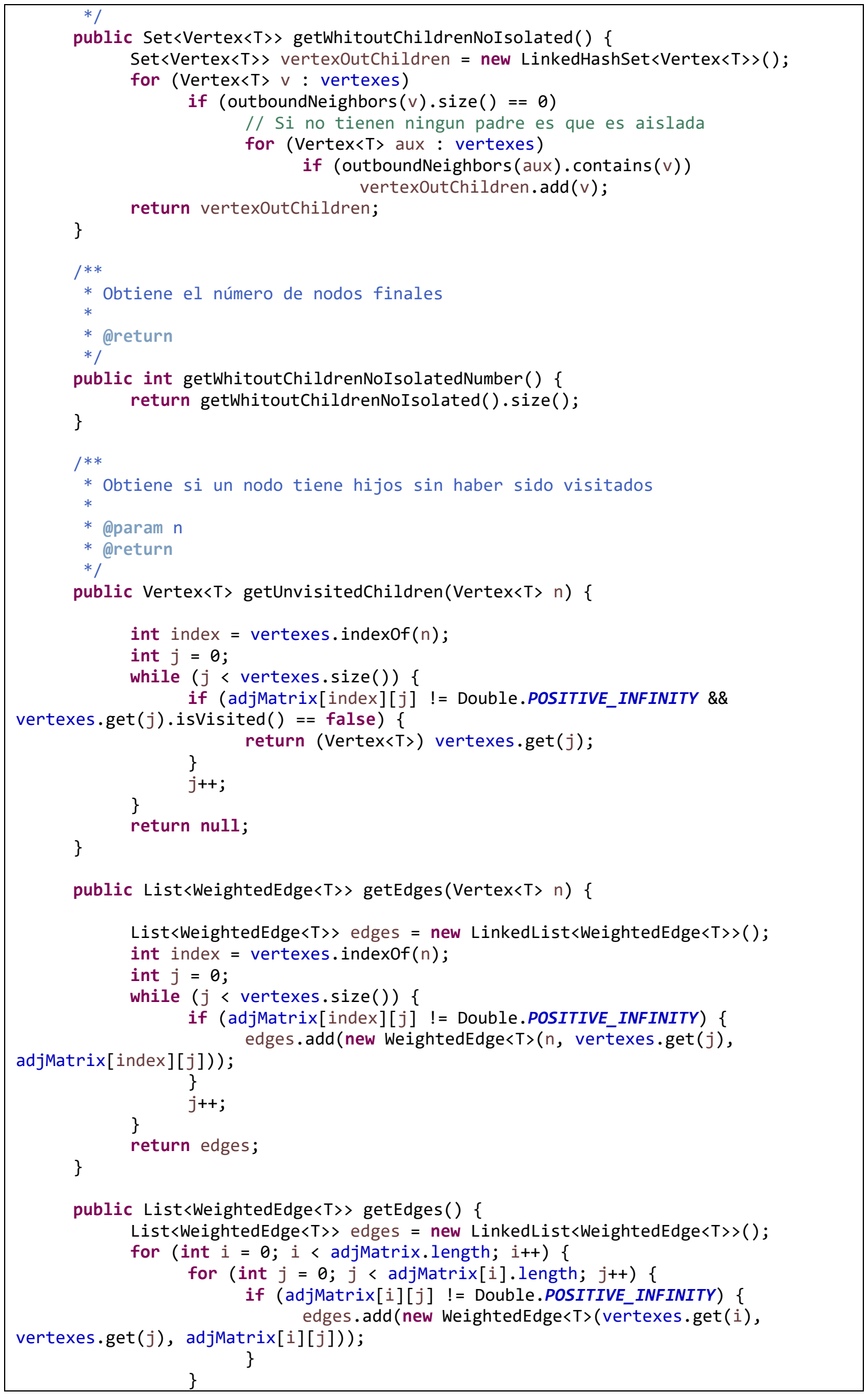




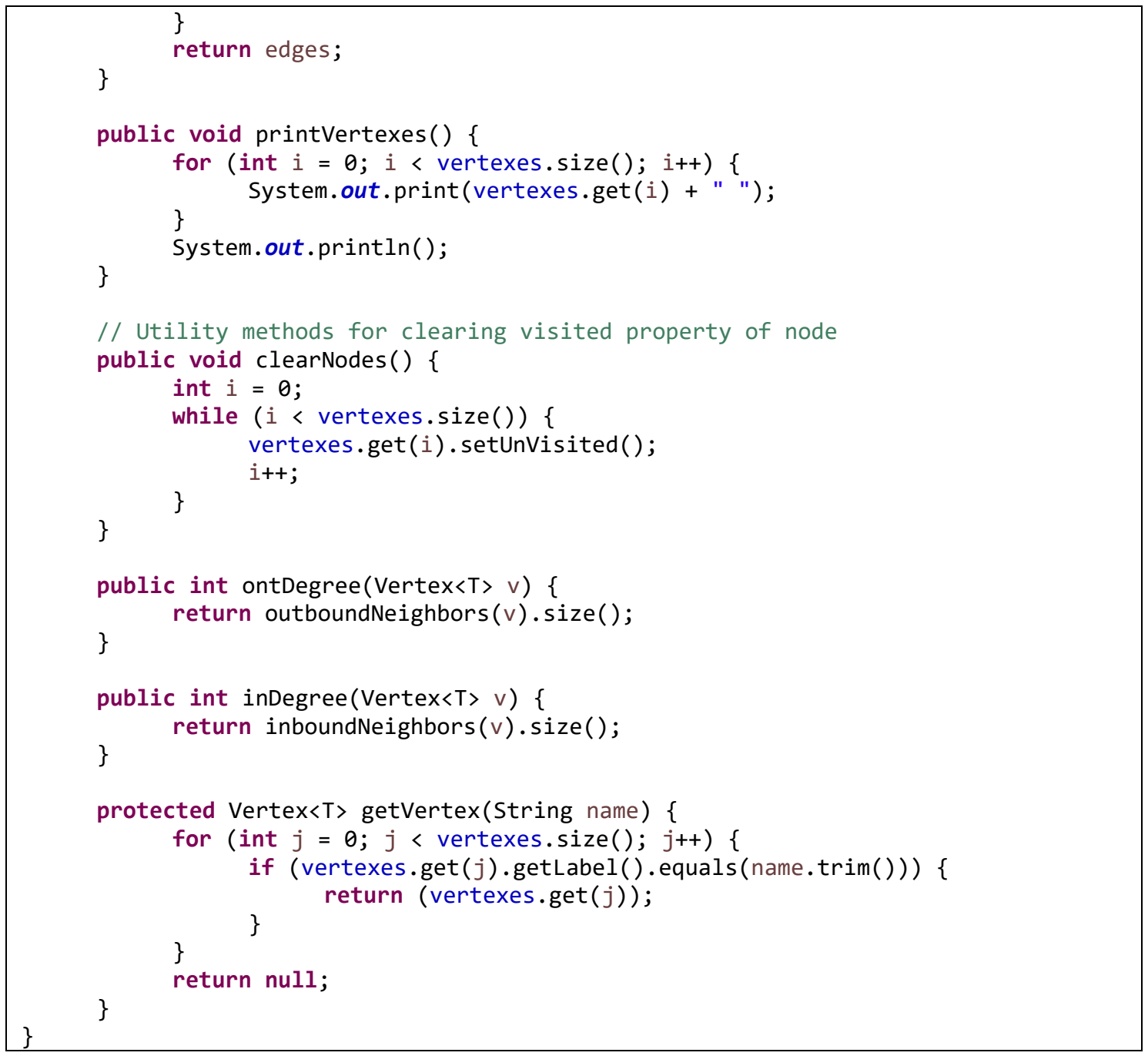

\section{Clase DirectedGraphFindAllPath $<\mathrm{T}>$.}

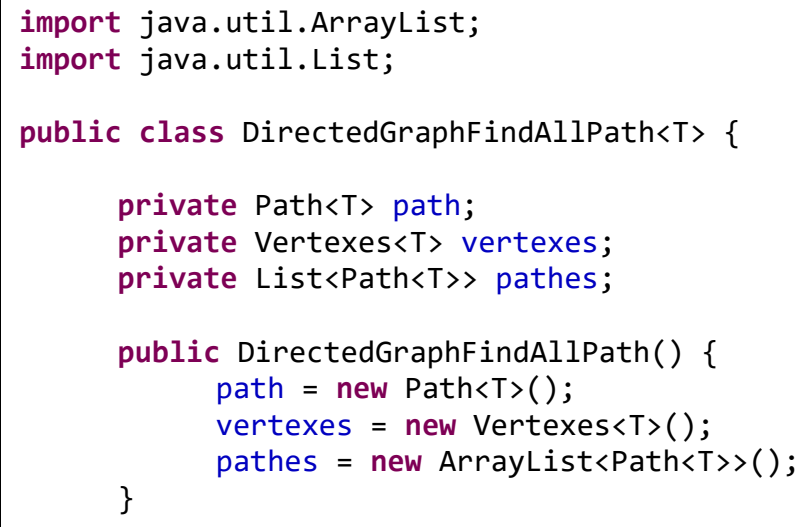




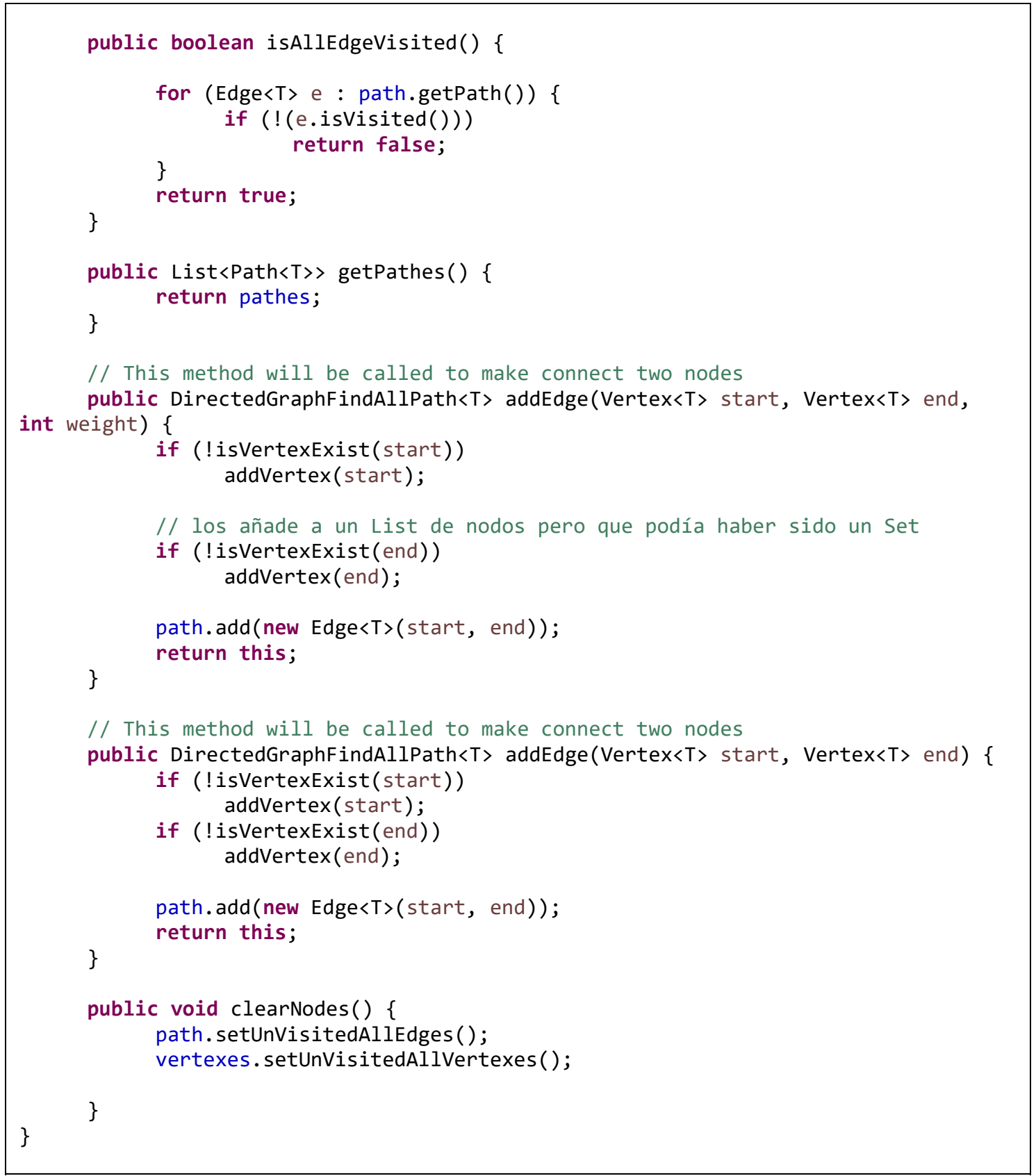

\section{Clase Edge.java $<T>$.}

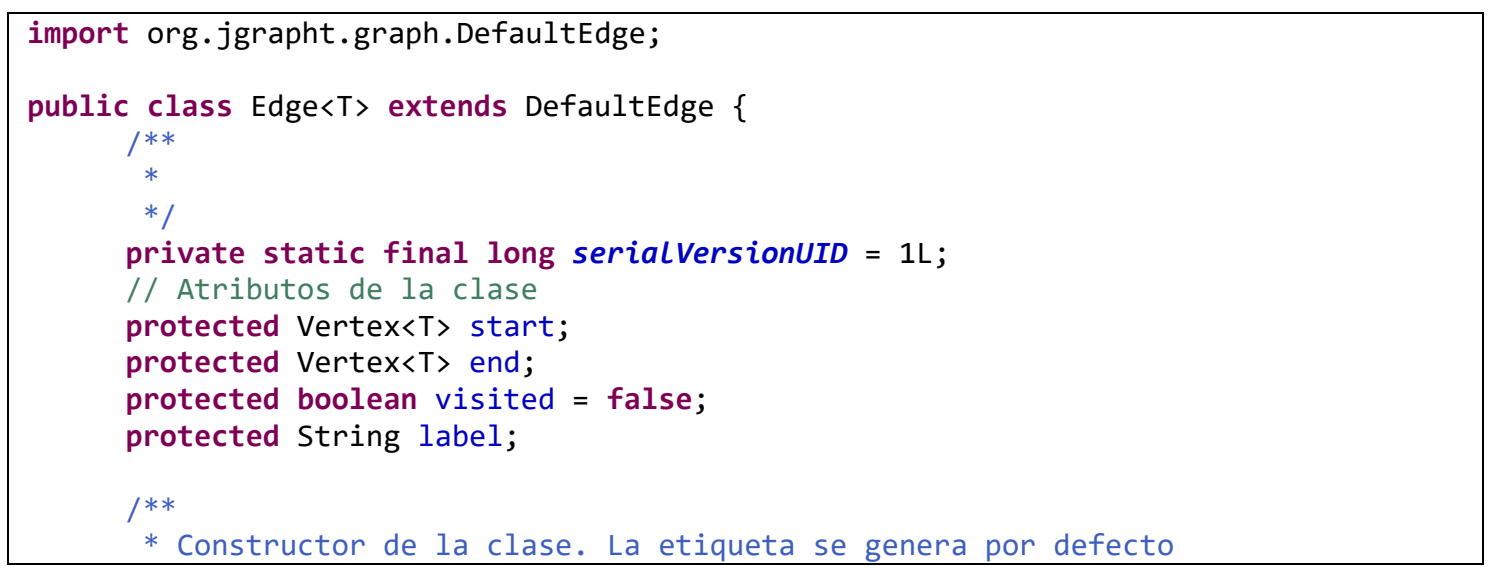




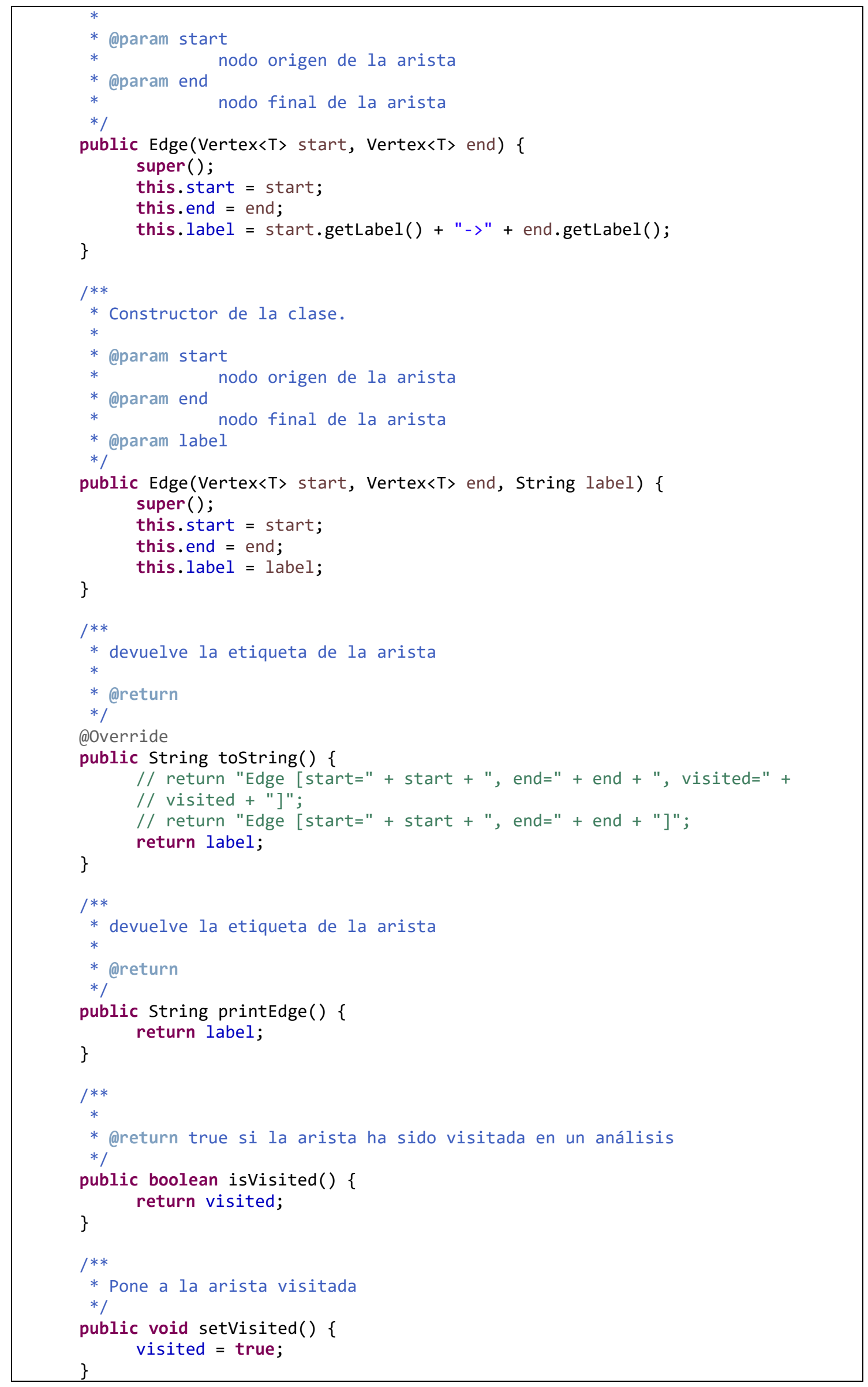




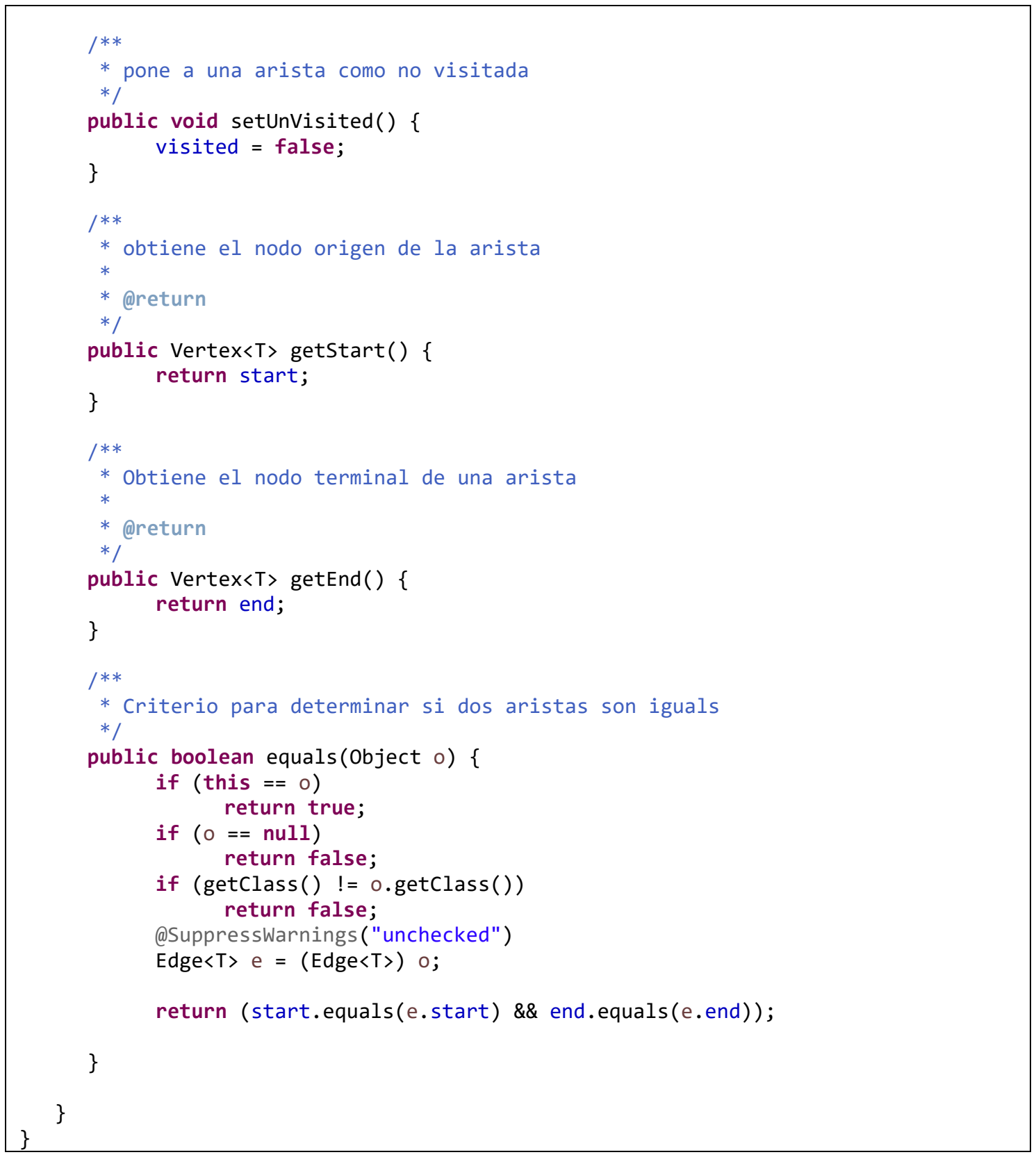

\section{Clase WeightedEdge $<T>$.}

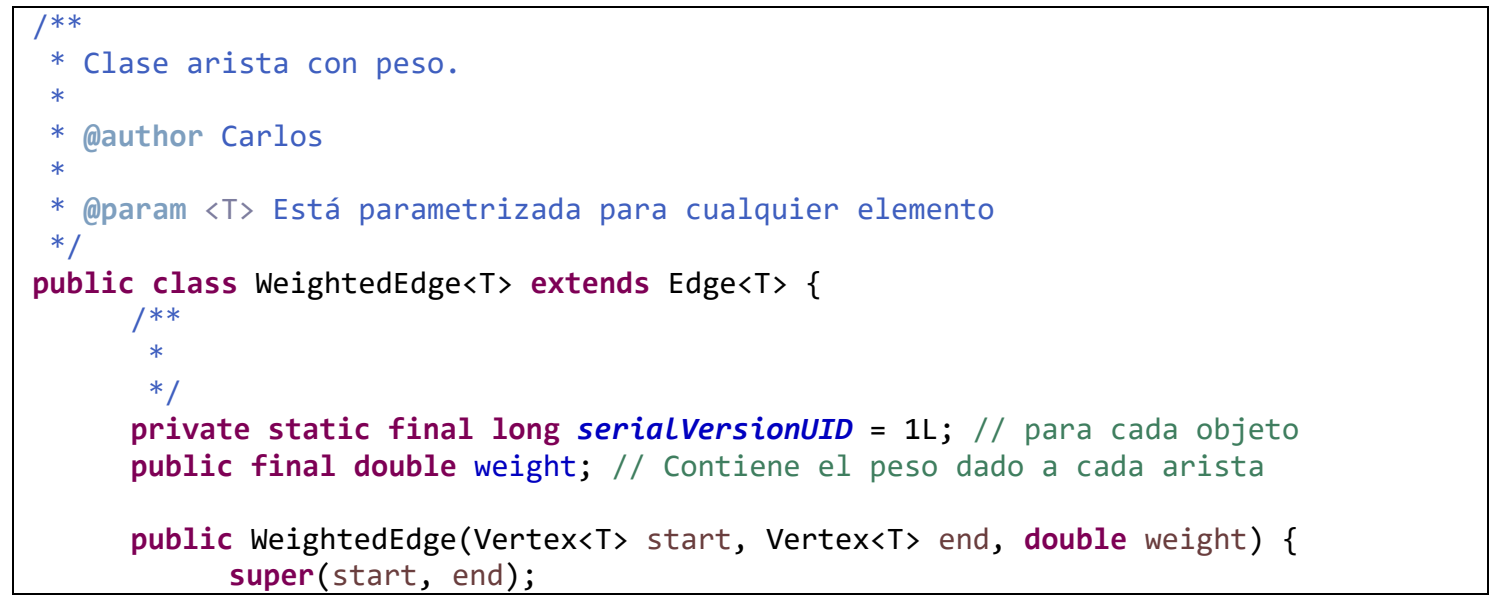




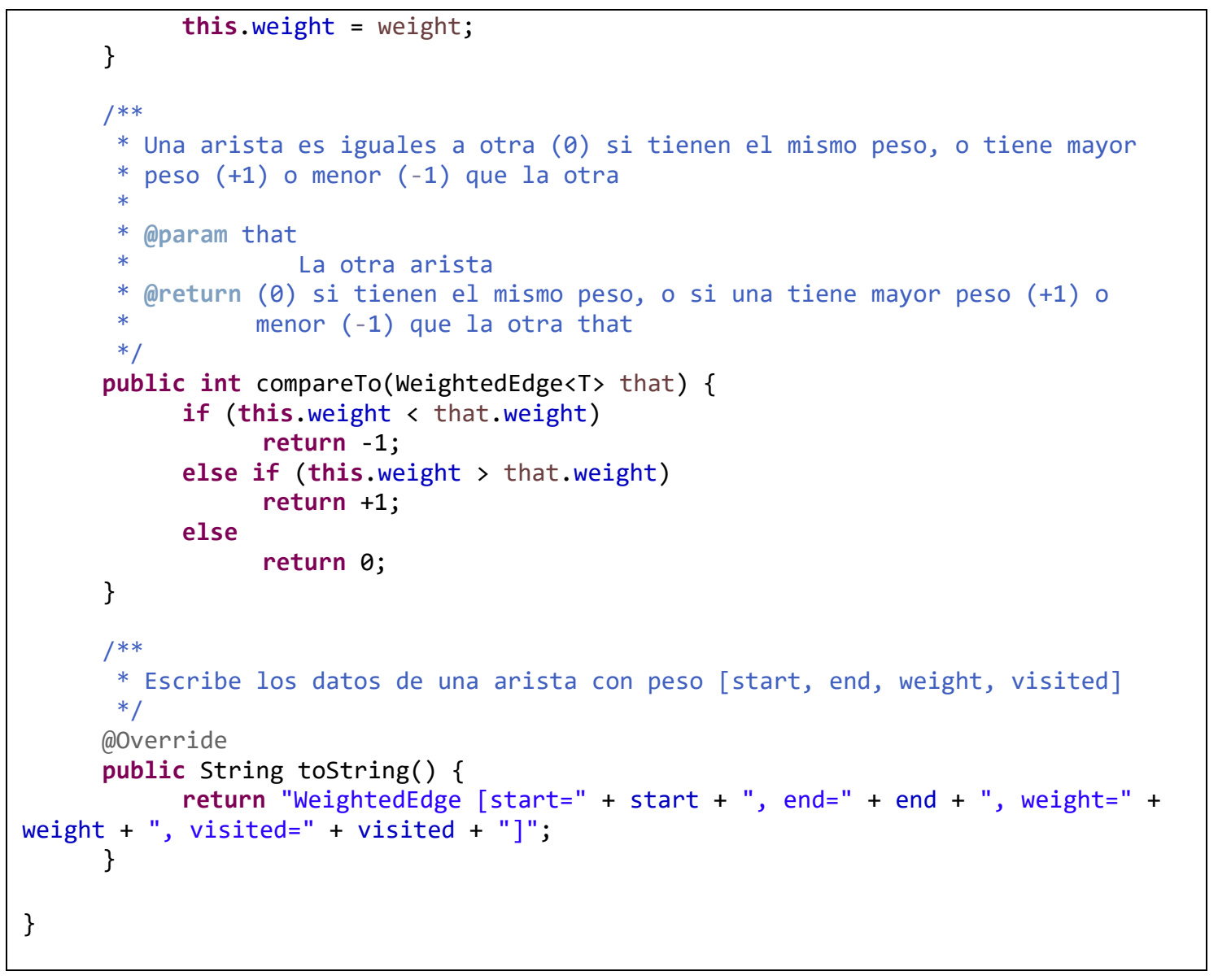

Clase Path $<\mathbf{T}>$.

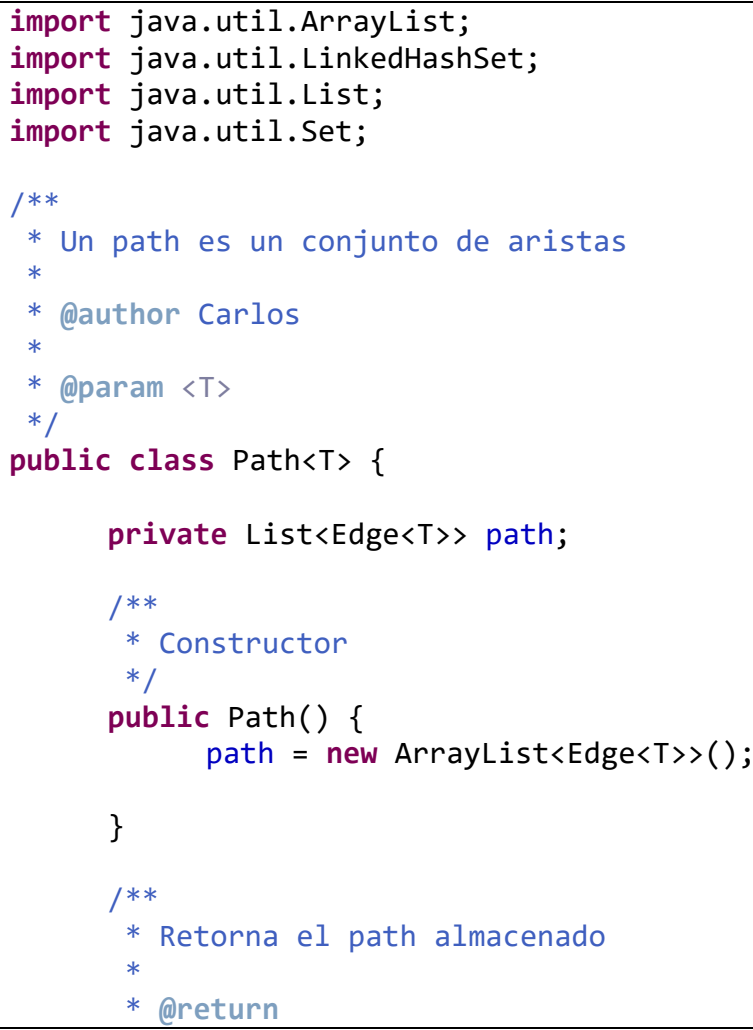




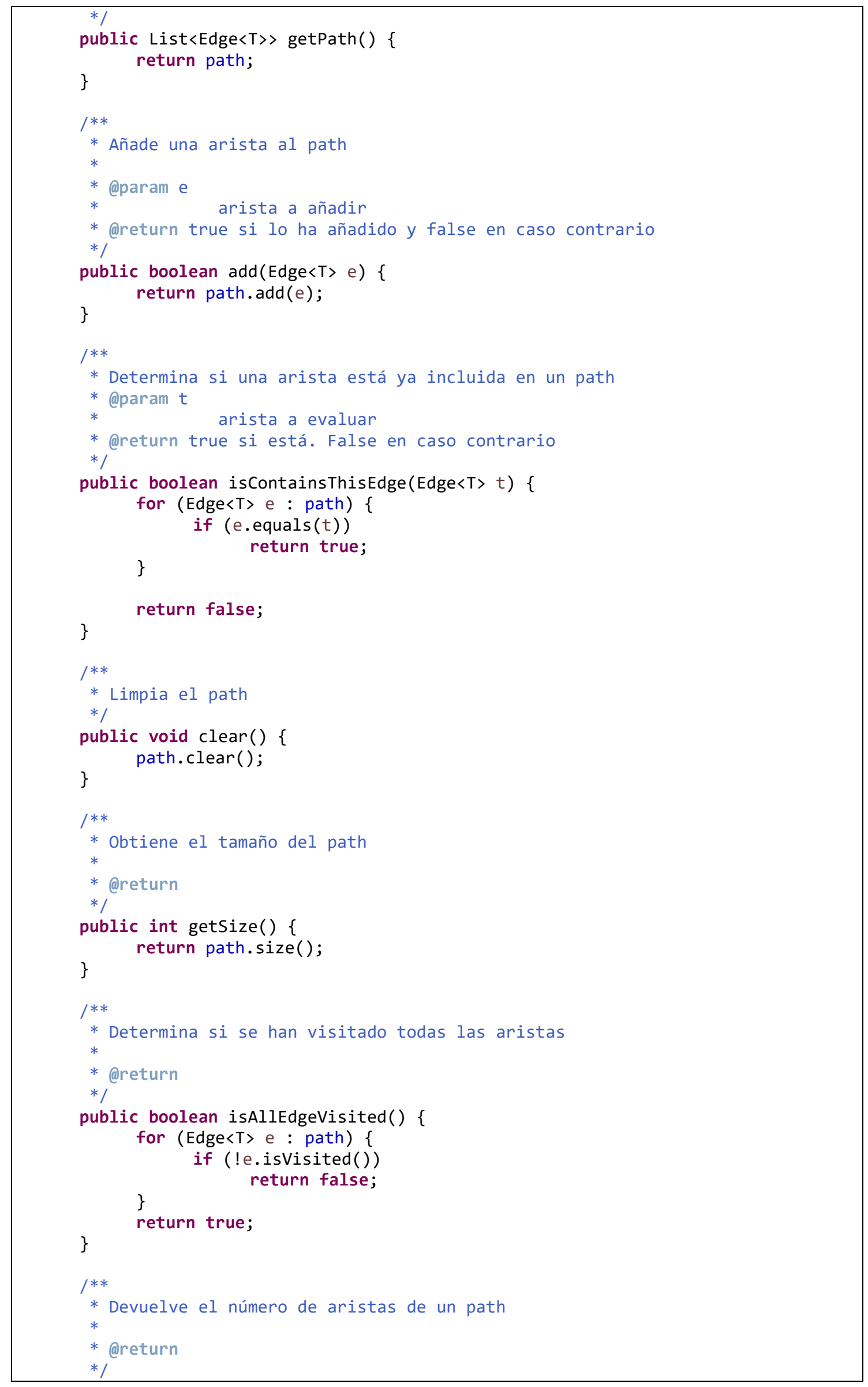




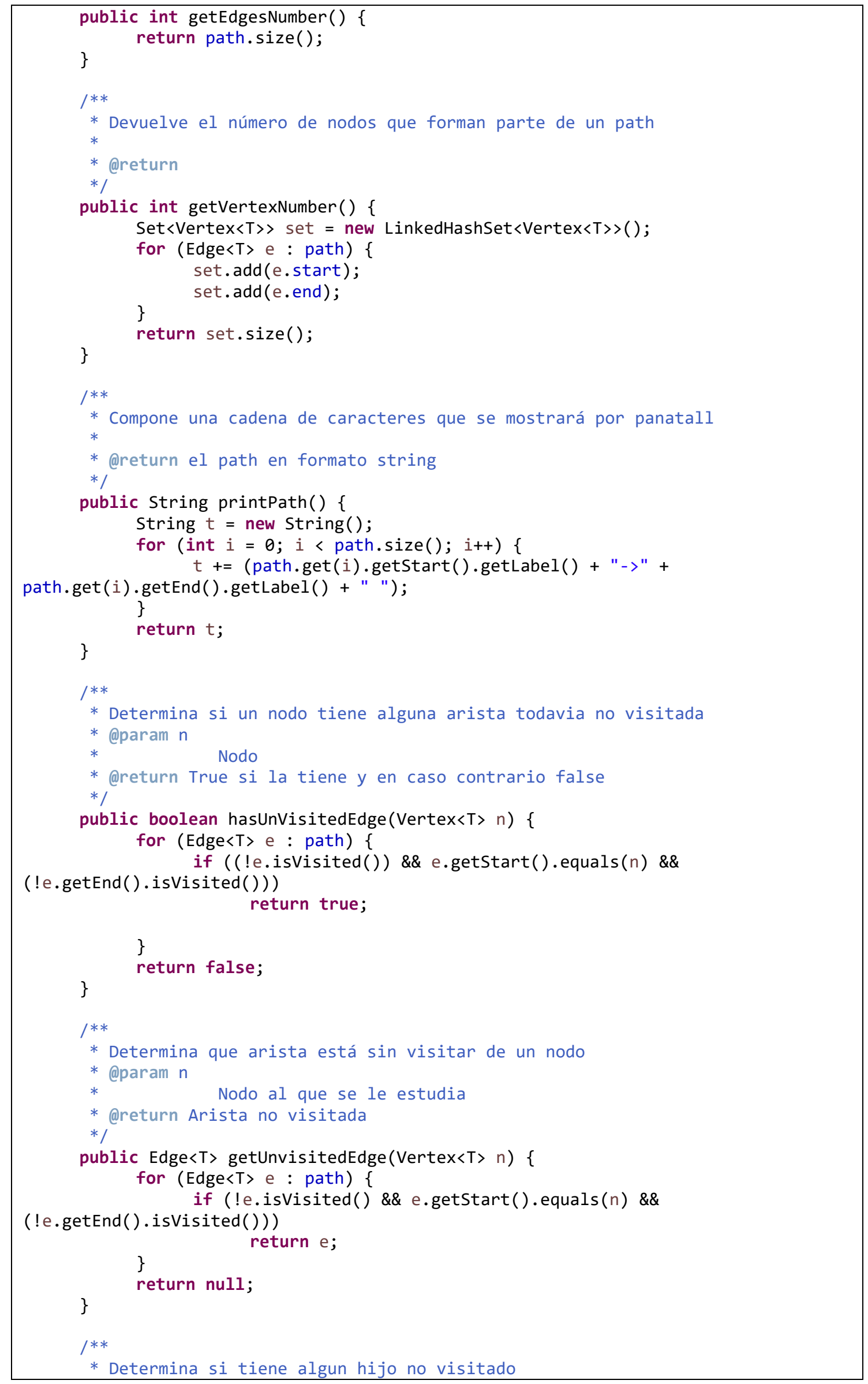




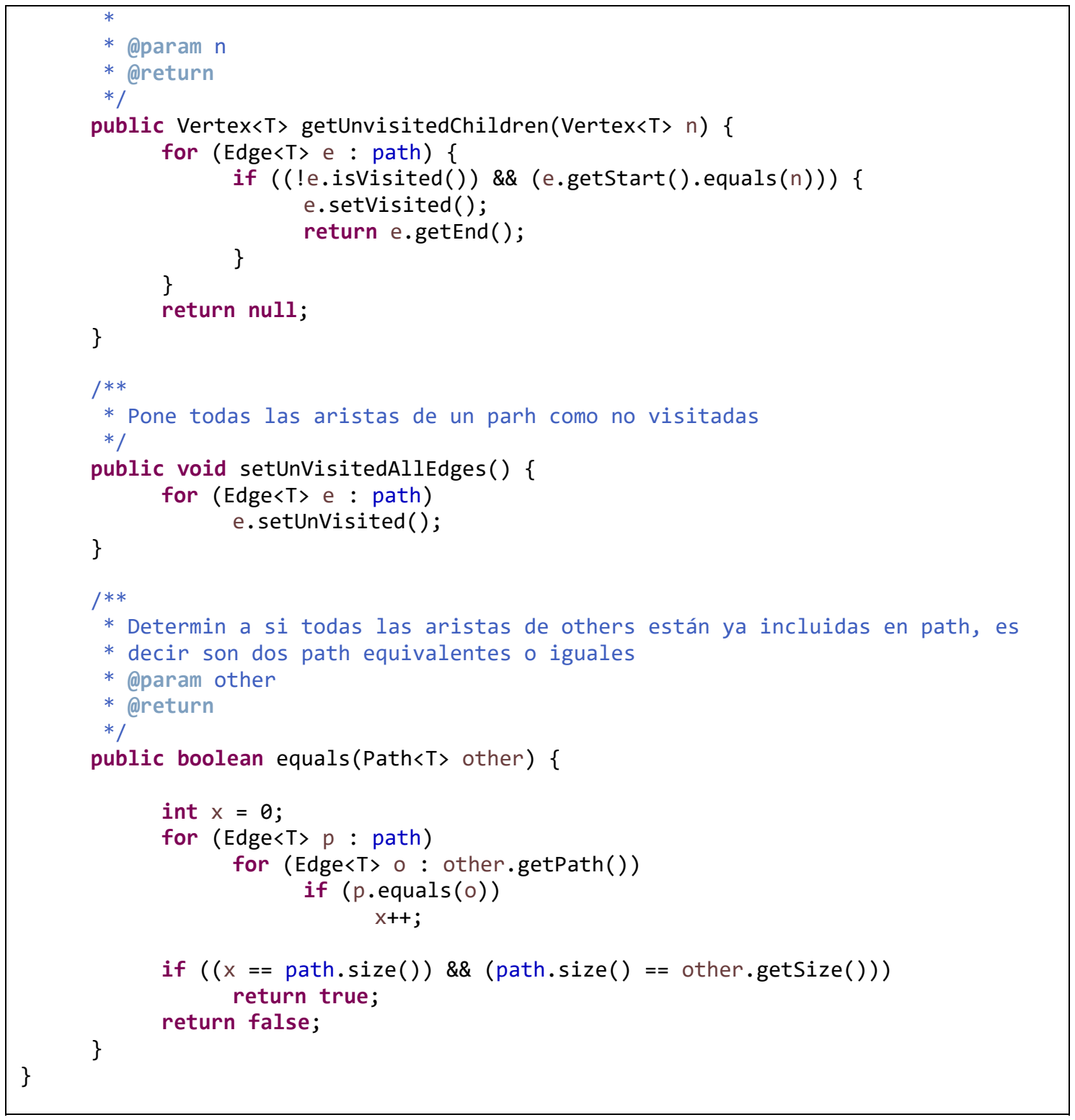

\section{Clase Vertex $<T>$.}

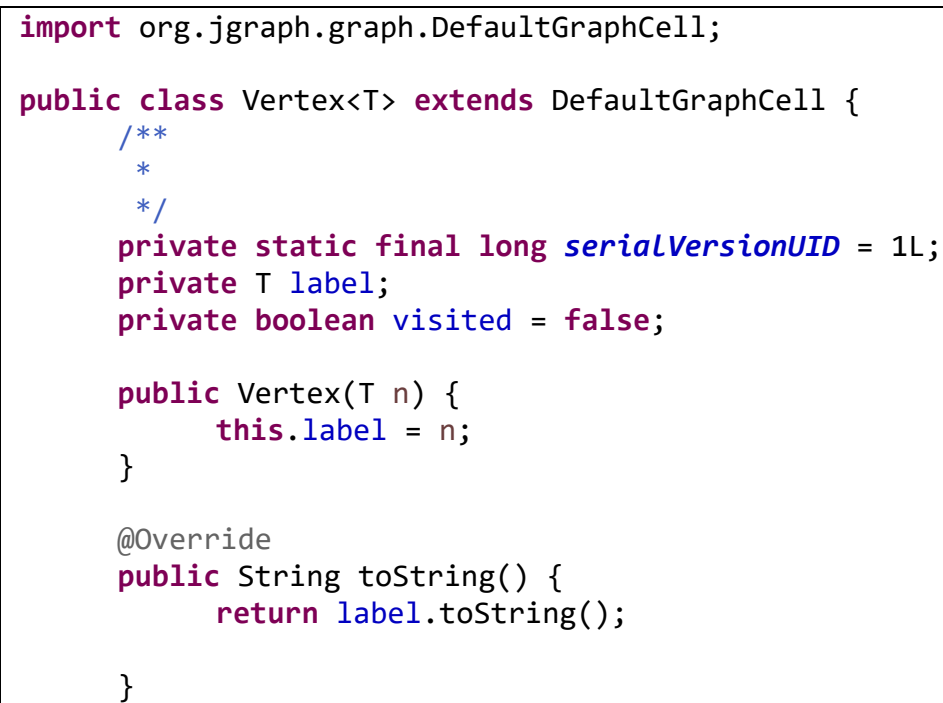




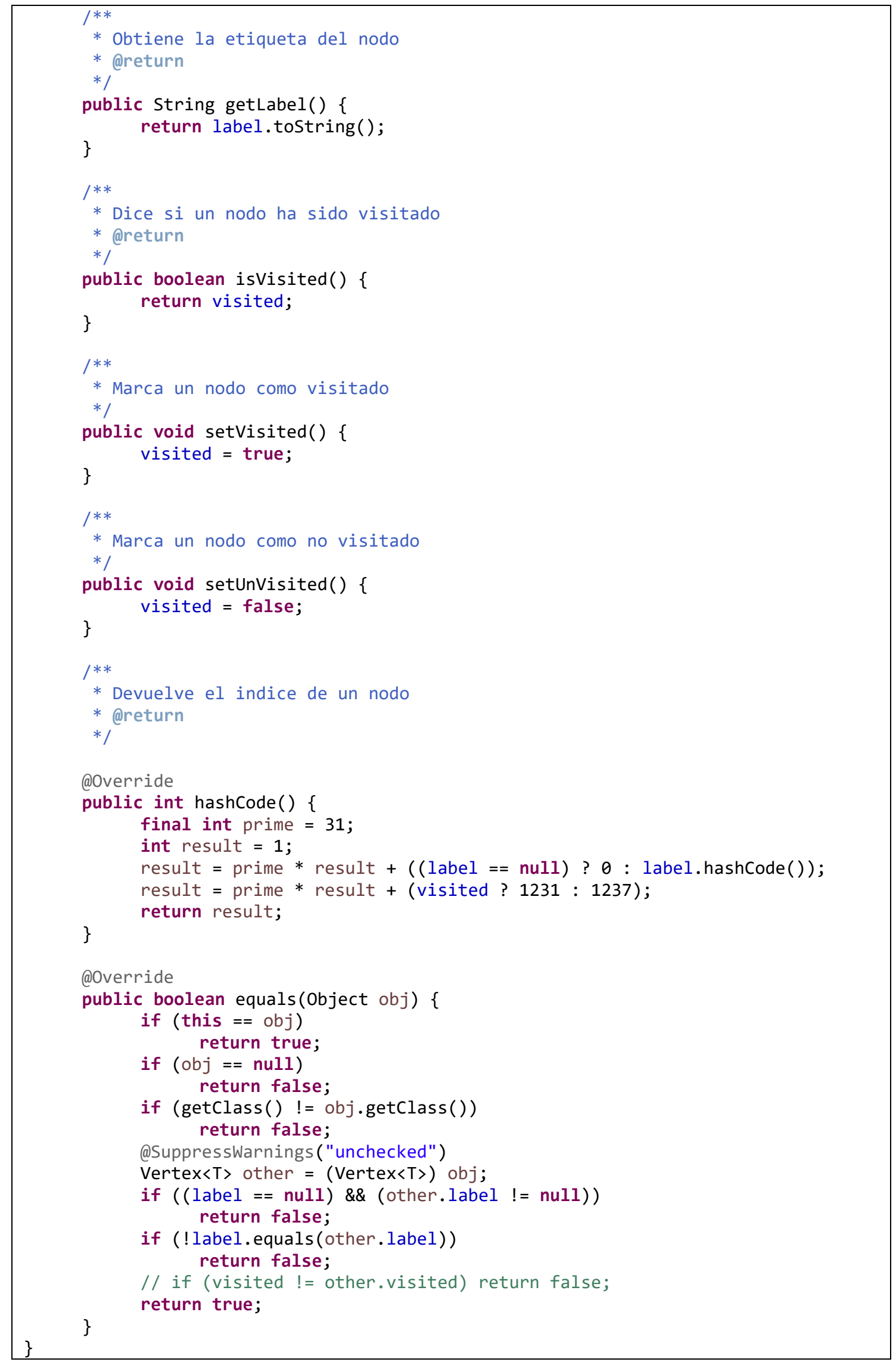




\section{Clase Vertexes $<\mathrm{T}>$.}

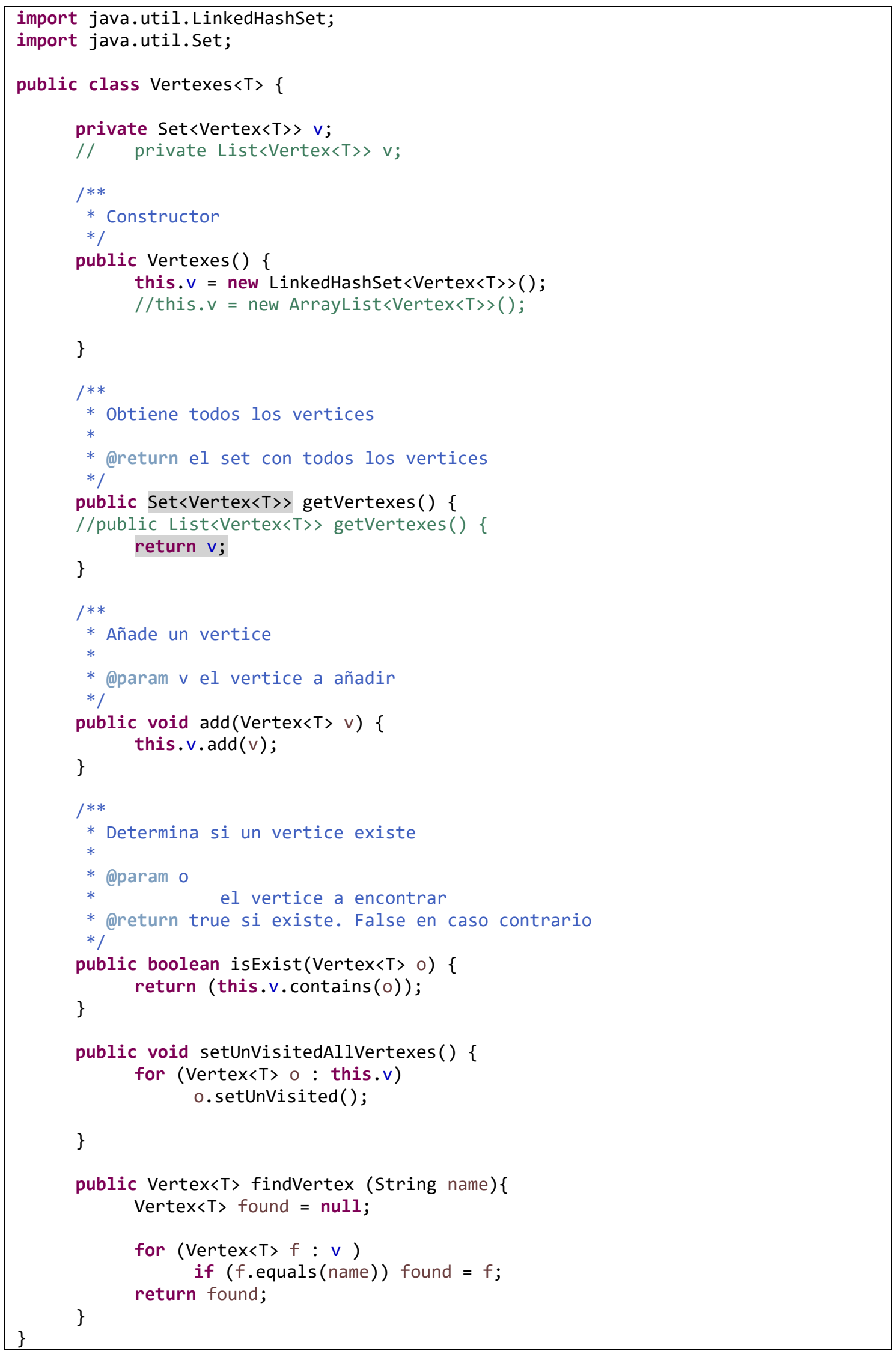




\section{Clase Messages.}

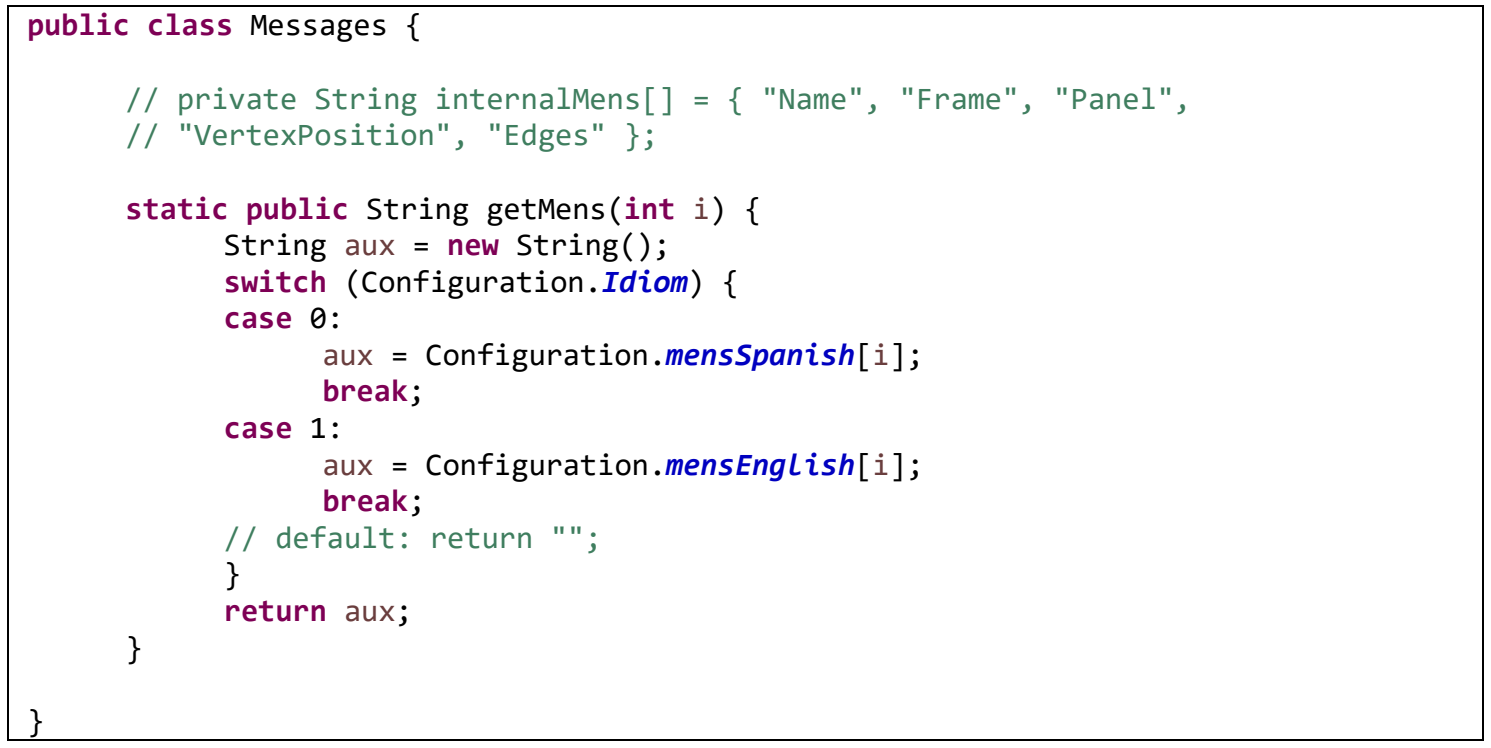

\section{Clase MetricException.}

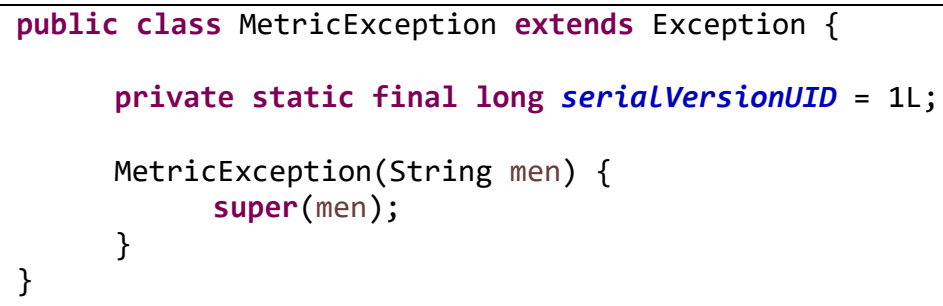

Contenido del fichero ServiceBasedInvocationsQOC.txt

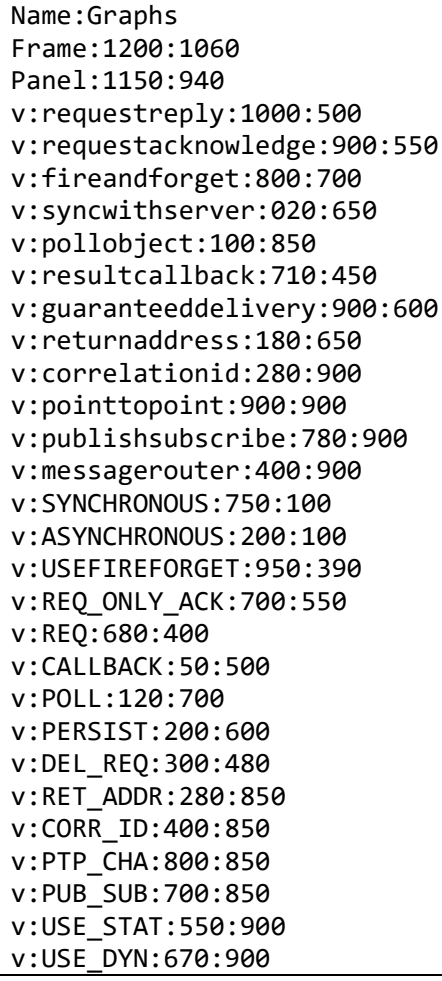




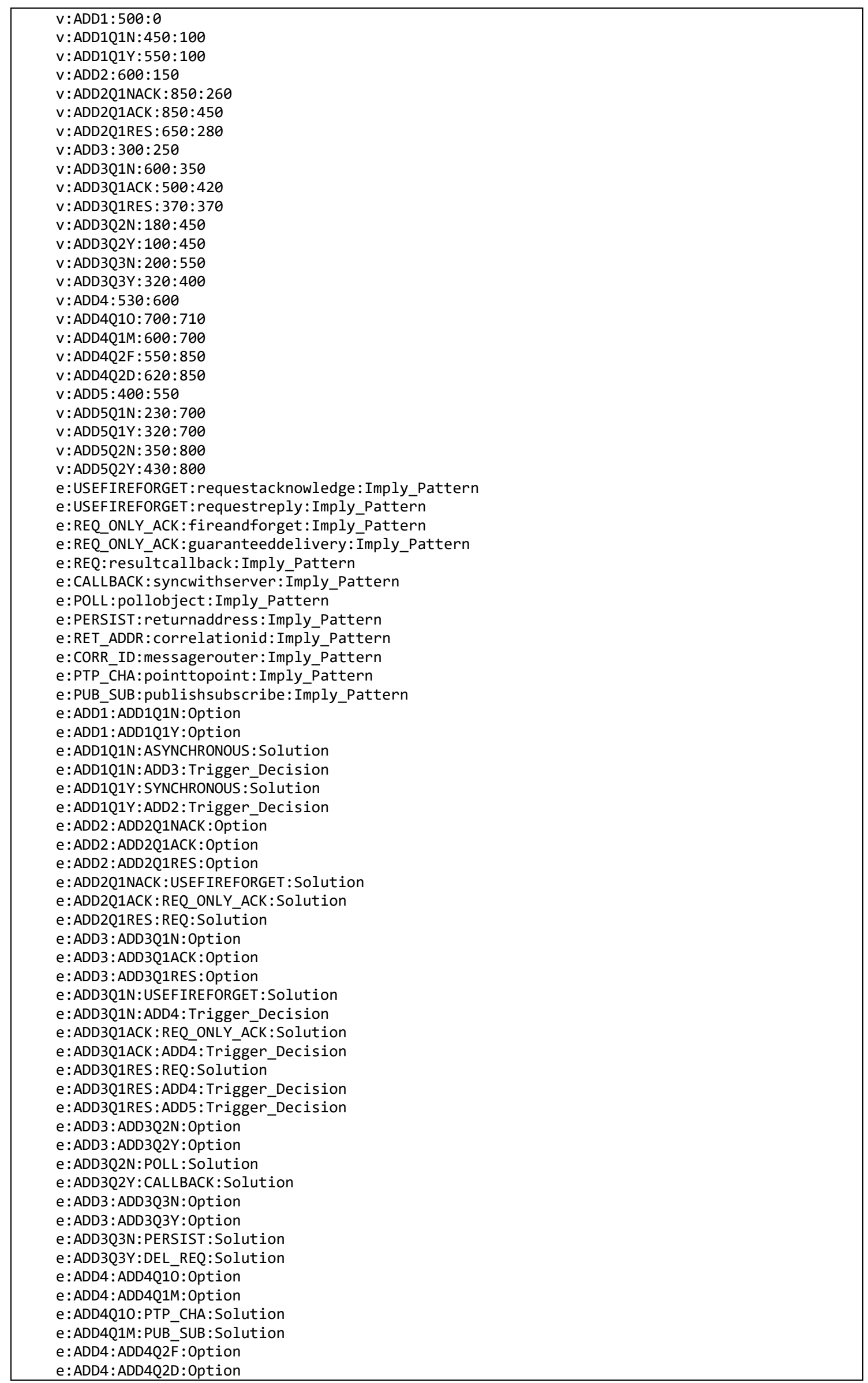


Anexo IV. Código de la aplicación $A D D N-M e t r i c s$

e:ADD4Q2F:USE_STAT:Solution

e:ADD4Q2D:USE_DYN:Solution

e:ADD5 : ADD5Q1N: Option

e:ADD5:ADD5Q1Y:Option

e:ADD5Q1Y:RET ADDR:Solution

e:ADD5: ADD5Q2N : Option

e: ADD5 : ADD5Q2Y:Option

e:ADD5Q2Y:CORR_ID:Solution 


\section{Anexo V. Preparación de datos para ADDN-Metrics}

En este anexo detallamos el formato del fichero de configuración que la aplicación $A D D N-M e t r i c s$ requiere para cargar los elementos arquitectónico de la red de decisiones como nodos y aristas. También indicaremos el proceso de adaptación que ha sido necesario realizar para las redes complejas $S B P 1$ y $S B P 2$, así como el fichero de configuración gráfica de la aplicación Neo4J.

Fichero de configuración: $A D D N-M e t r i c s$ permite visualizar la red de decisiones a partir de la información detallada en un fichero de configuración. Cada nodo y arista está identificado visualmente por un nombre. La aplicación permite determinar en qué posición espacial se situará cada nodo, dentro de un panel JAVA. Si no indicamos su posición, la aplicación lo situará en una posición por defecto permitiendo al usuario mover el nodo de posición posteriormente. A continuación, en la tabla V.1, exponemos el formato de este fichero.

Tabla V.1. Formato del fichero de configuración de una red de decisiones

\begin{tabular}{|l|l|}
\hline Definición & Explicación \\
\hline Name: NombreRedDecision & Es el nombre de la red de decisiones. \\
\hline Frame: Ancho:Alto & $\begin{array}{l}\text { Ancho y alto de la ventana donde se visualiza la red separados } \\
\text { por el carácter “:”. }\end{array}$ \\
\hline Panel: Ancho:Alto & $\begin{array}{l}\text { Ancho y alto del panel donde se ubicara la red, dentro de la } \\
\text { ventana de visualización separados por el carácter “:”. }\end{array}$ \\
\hline $\mathbf{v}: \mathrm{DD}_{\mathrm{i}}: \mathrm{POSX}_{\mathrm{i}}: \mathrm{POSY}_{\mathrm{i}}$ & $\begin{array}{l}\text { Por cada nodo, y separado por “:” (dos puntos) se indicará que } \\
\text { es un nodo, el identificador de la decisión, su posición (X,Y) } \\
\text { dentro del panel. }\end{array}$ \\
\hline e:DD $\mathrm{D}_{\mathrm{i}}: \mathrm{PD}_{\mathrm{k}}:$ Nombre_arista & $\begin{array}{l}\text { Por cada arista, y separado por “:” (dos puntos) se indicará que } \\
\text { es arista (egde o "e”) y qué dos decisiones enlaza. } \\
\text { Opcionalmente aparece el nombre de la arista Además, estas } \\
\text { dos decisiones tienen que existir. }\end{array}$ \\
\hline
\end{tabular}

La primera línea indica el nombre de la red. A continuación, especificamos el tamaño de la ventana Java donde mostraremos la red y aquella información que se considere de interés. La red de ubicará en el panel cuya dimensión se especifica en la tercera línea. A 
continuación, habrá tantas líneas como decisiones exista, y posteriormente todas las trazas que unan decisiones.

Como ejemplo de este formato, en la tabla VI.2 detallamos la configuración del modelo “Model 1" propuesto en la sección 6.1.1 y cuyo nombre (Name) especificamos en la línea 1. Esta red se representa en una ventana (Frame) de 230 pixeles de ancho por 190 de alto (línea 2), dentro de un panel (Panel) de tamaño 200 pixeles de ancho por 70 de alto (línea 3). La decisión “DD0" (identificada por $v$ ) la ubicamos en la posición 30,30 (línea 4) y la decisión DD1 (identificada por v) en la posición 90,30 (línea 5) del panel. Por último, existe una única traza (identificada por $e$ ) que enlaza unidireccionalmente la decisión DD0 con DD1 (línea 6). En el espacio libre desde el panel hasta el fin de la ventana ubicamos mensajes de la aplicación como es el valor de la complejidad calculada. También colocamos un botón cuyo efecto es cerrar la ventana.

Tabla V.2. Ejemplo de configuración de una red de decisiones.

\begin{tabular}{|c|c|c|c|}
\hline Contenido & Represe & ción gráfica & \\
\hline $\begin{array}{l}\text { Name:Model } 1 \\
\text { Frame:230:190 } \\
\text { Panel:200:70 } \\
\text { v:DD0:30:30 } \\
\text { v:DD1:90:30 } \\
\text { e:DD0:DD1 }\end{array}$ & $\begin{array}{l}>1 \\
\frac{\pi}{0} \\
0 \\
0 \\
0 \\
0 \\
0 \\
0 \\
0 \\
0\end{array}$ & $\begin{array}{l}\text { DD0 DD1 } \\
\text { Complexity: } 0.67 \\
\quad \text { Close }\end{array}$ & \begin{tabular}{l|l}
$\square$ & $\times$ \\
Coordenada X
\end{tabular} \\
\hline
\end{tabular}

A partir del nombre de cada decisión y de cada relación existente en este tipo de fichero ASCII, ADDN-Metrics genera el grafo.

\section{Adaptación de datos de redes complejas:}

El formato de datos proporcionado es ".json". Ha sido necesario transformar los datos identificando para ello cada decisión y cada relación a través de la información existente, hasta llegar a la visualización de las redes. Este proceso de adaptación la detallamos en la figura V.1. 


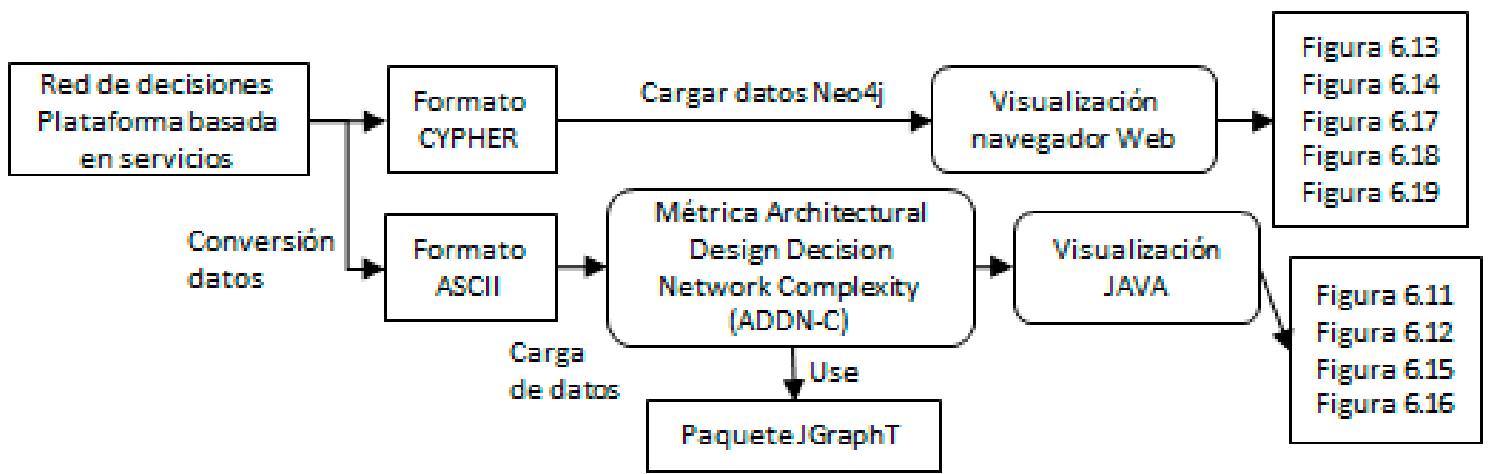

Figura V.1: Proceso de adaptación de datos

Este proceso de adaptación ha sido dual, donde el primero de ellos está orientado a la correcta interpretación de los datos por parte de la aplicación Neo4j, y el segundo enfocado a $A D D N-$ Metrics

En las tablas V.3 y V.4 mostramos un ejemplo de cómo están declaradas dos decisiones y una relación respectivamente, en formato “.json”. Las decisiones están definidas como elementos del vector elements [] . Cada decisión está identifica por los campos "id", "name" y "description", además de la posición donde está ubicada en dicho vector.

Tabla V.3. Ejemplo de dos decisiones codificadas en formato .json.

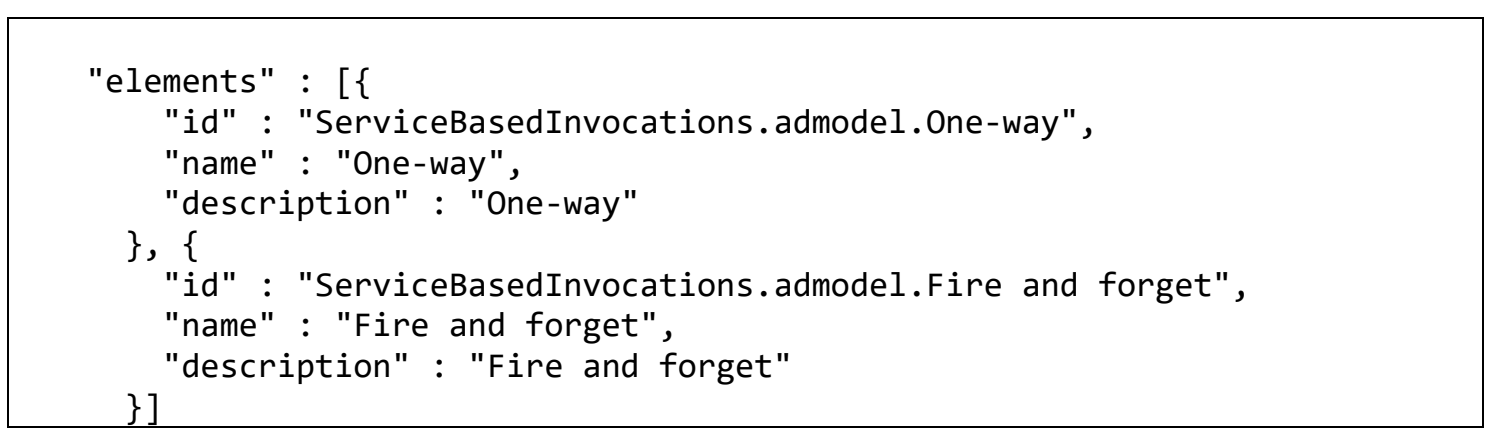

Las relaciones entre decisiones también forman parte del vector elements [ ] y están definidas por los campos "id" y "name". Cada relación tiene un vector llamado options [] cuyos campos "\$ref" identifican cada una de las decisiones que relaciona, y su contenido identifica la posición que la decisión relacionada ocupa en el vector elements[]. El resto de campos no son relevantes para nuestra aplicación. 
Tabla V.4. Ejemplo de una relación codificadas en formato .json

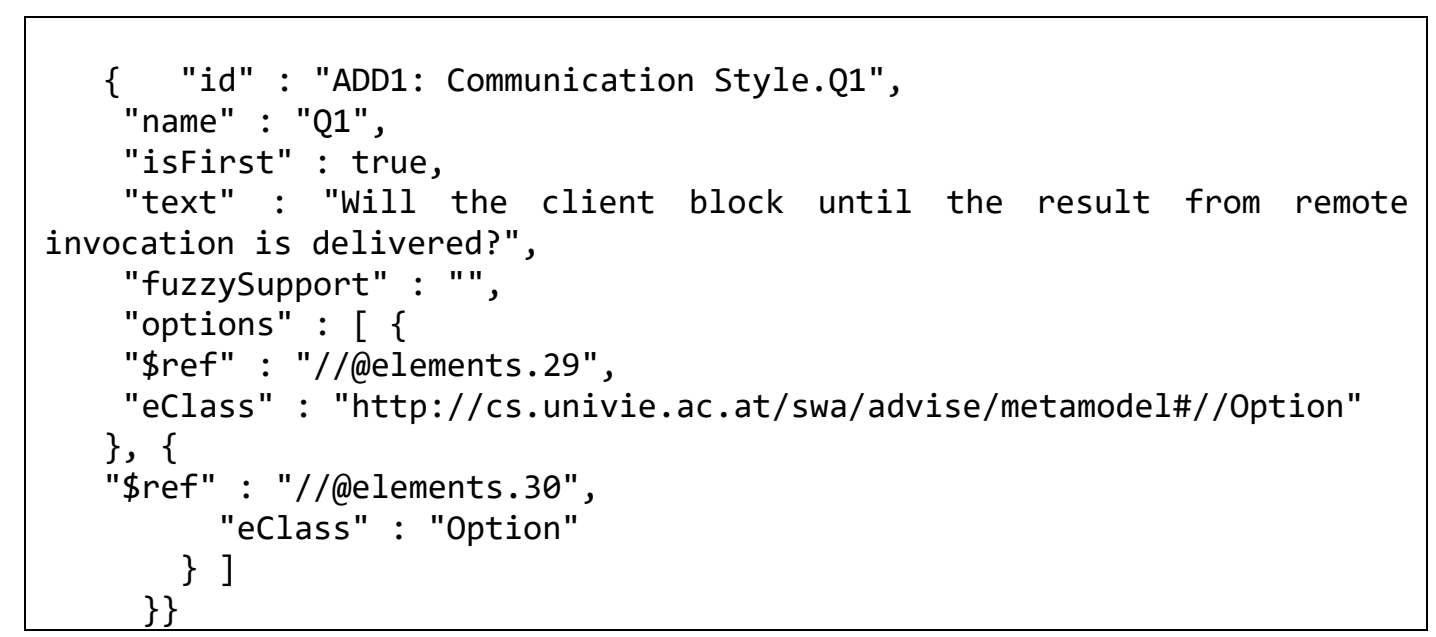

El resultado de conversión de los datos existentes en las tablas V.3 y V.4 a nuestro formato ASCII lo mostramos en la tabla V.5.

Tabla V.5. Ejemplo de una relación codificadas en formato .txt

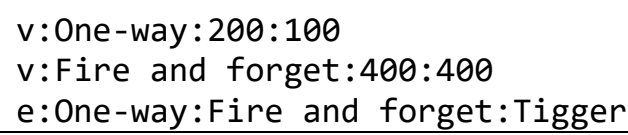

En el caso de las dos redes de decisiones complejas utilizadas en este trabajo, hemos tenido que utilizar la aplicación $\mathrm{Neo} 4 \mathrm{j}$ para representar la red de decisiones por su elevado número de decisiones. Como ya hemos indicado anteriormente, consideramos que especificar la posición espacial de un número elevado de nodos es una tarea que excede a las tareas de un arquitecto software. Por ello, hemos transformado los datos desde el formato ".json" al lenguaje nativo Cypher. Tomando como origen los ficheros ".json" facilitados (un extracto lo mostramos en las tablas V.3 y V.4) hemos obtenido los datos identificativos de cada decisión para pasarlos como argumentos del mandato CREATE (ver tabla V.6) para crear sendos nodos.

Tabla V.6. Ejemplo de un nodo codificadas en formato .CYPHER

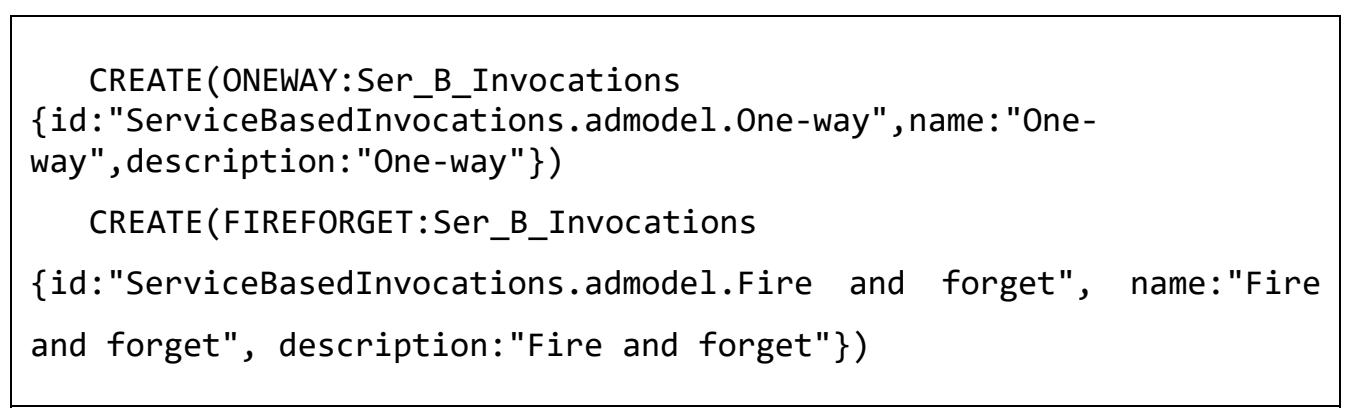


En cuanto a las relaciones, también ha sido necesario localizar y los elementos identificativos de las relaciones y adaptarla al formato ".CYPHER" que la aplicación Neo4j requiere, como detallamos en la tabla V.7.

Tabla V.7. Ejemplo de una relación codificadas en formato CYPHER

CREATE (ONEWAY)-[:Trigger_Questions] -> (FIREFORGET)

\section{Fichero de configuración visual de la aplicación Neo4j.}

Para la correcta visualización de un grafo en Neo4j, la aplicación permite configurar la representación del grafo mediante la programación de un fichero con extensión “.grass” (graph-style-sheet). En este fichero se especifica el tipo de letra, color tamaño y etiquetas tanto de nodos como de las relaciones. En nuestro trabajo ha sido necesario programarlo para una correcta visualización. A continuación, en la tabla visualizamos el contenido del fichero de configuración "graphstyle.grass" empleado en nuestra experimentación.

Tabla V.8. Contenido del fichero de configuración "graphstyle.grass" de Neo4j.

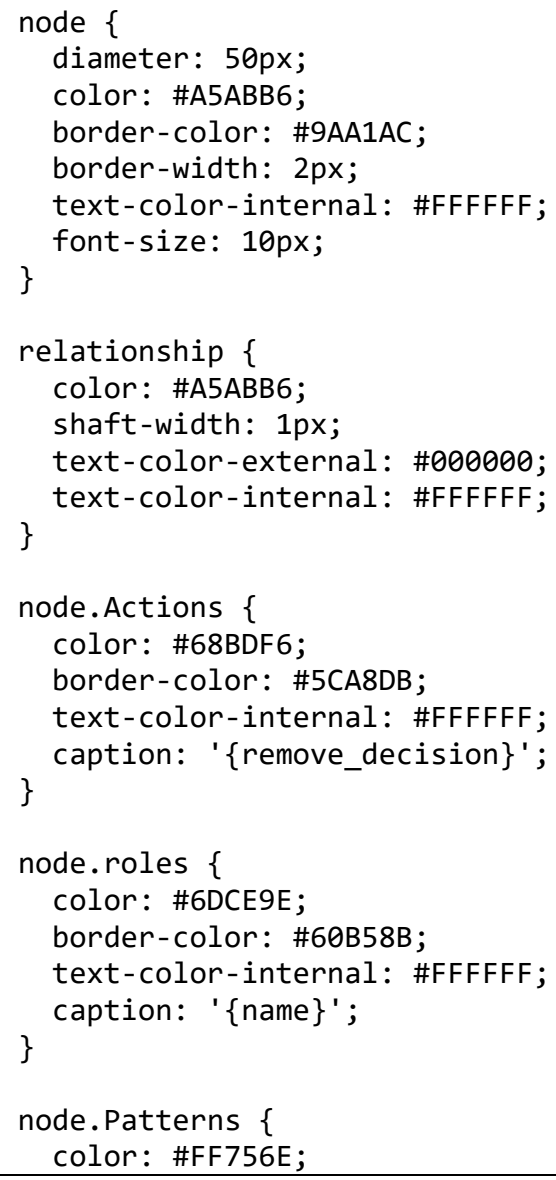




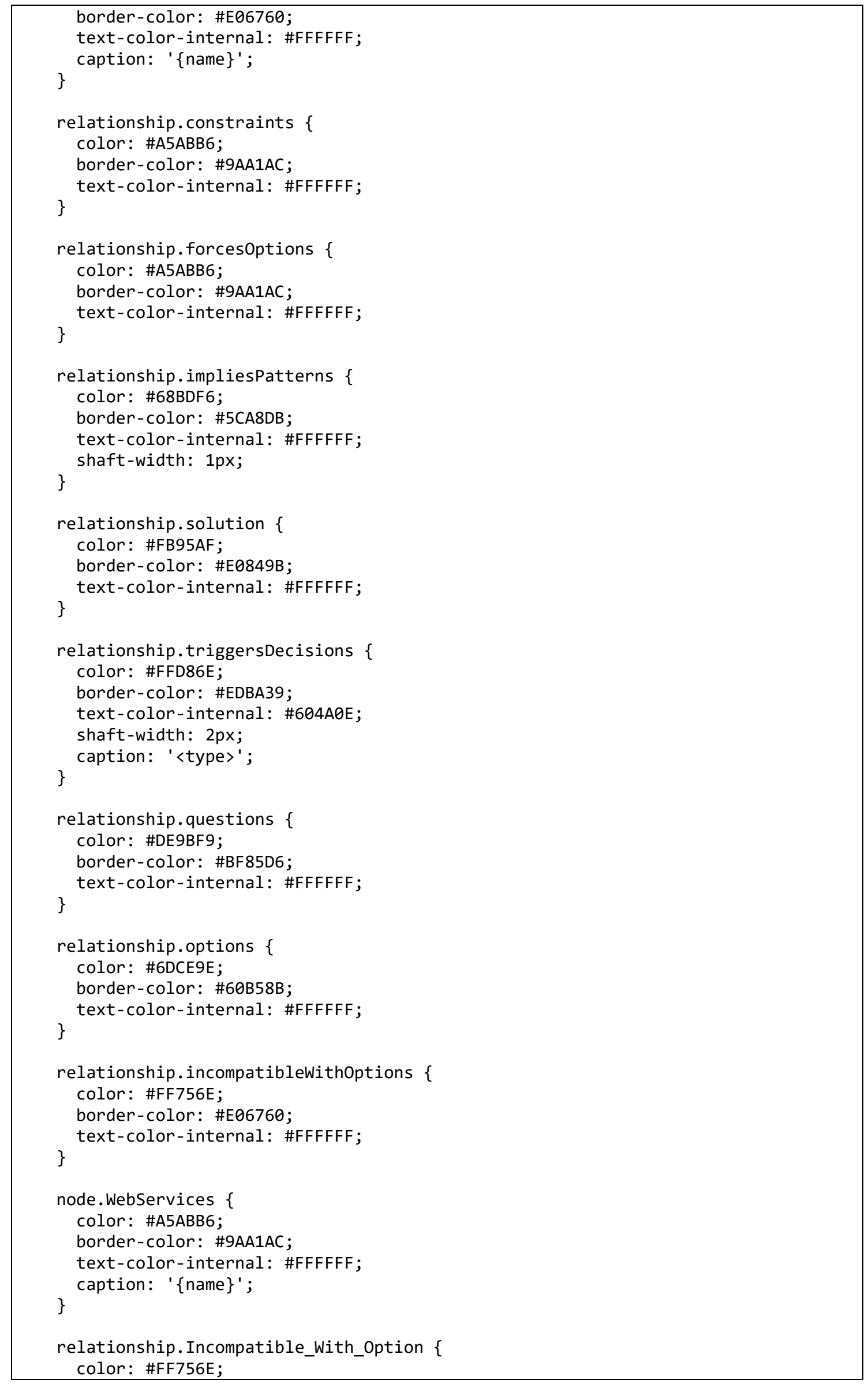


Anexo V. Preparación de datos para ADDN-Metrics

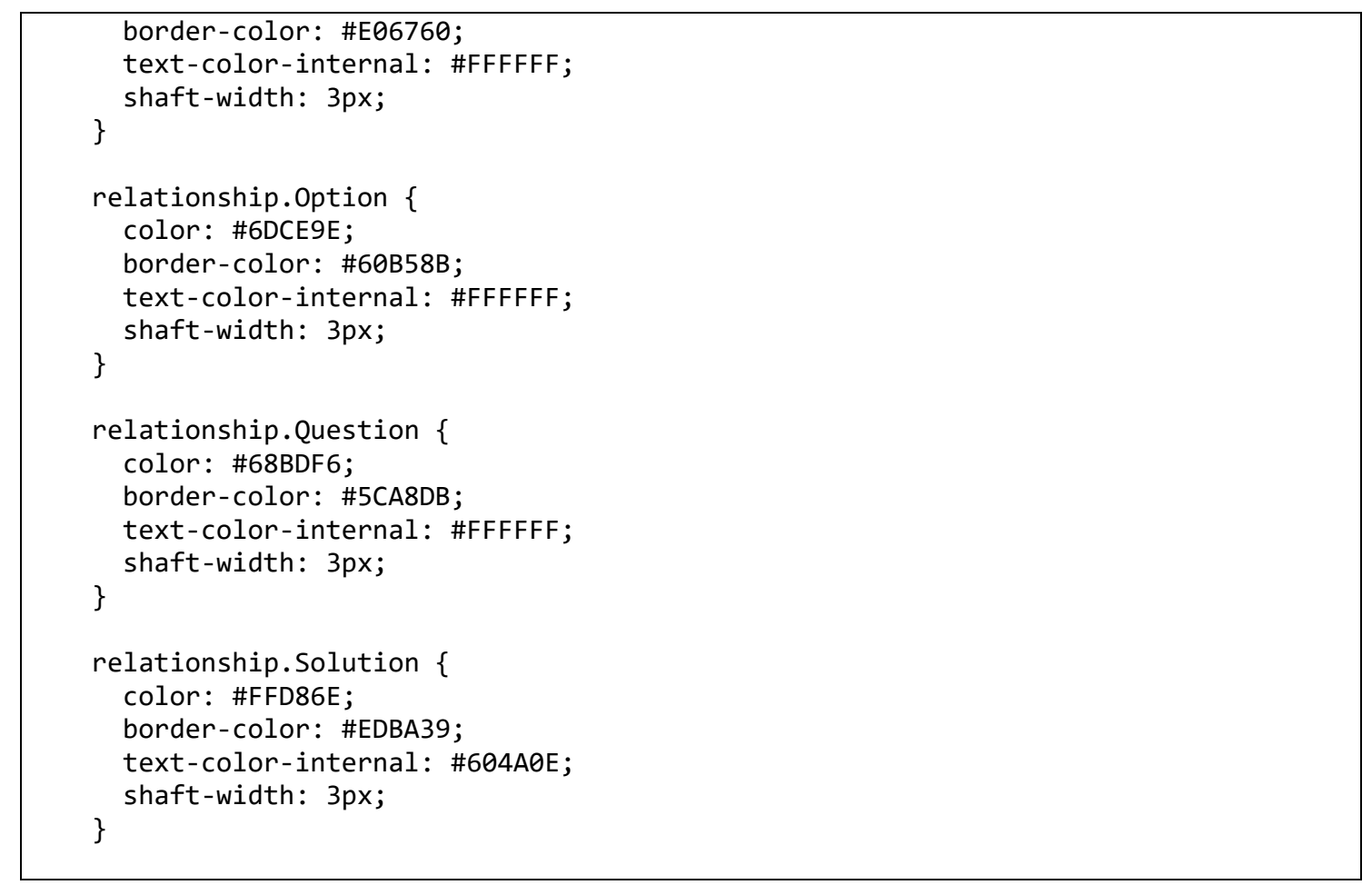




\section{Anexo VI. Experimento URJC}

El experimento al que se hace referencia en la sección 5.2 está formado por un enunciado de requisitos para el diseño de una aplicación software. Para capturar las decisiones de diseño disponemos de tres plantillas. Una vez finalizado la fase de captura de decisiones, procedemos a entrevistar a los diferentes miembros de este experimentos para solicitarles información adicional para poder resolver las preguntas RQ1, RQ2 y RQ3 planteadas en la sección 5.2.2 de este trabajo.

A continuación, detallamos el enunciado de requisitos, así como el resto de material empleado en este experimento.

\section{Diseño de una Arquitectura Software}

Se necesita diseñar un sistema complejo de emergencias (SCE), el cual comprende una interfaz centralizada de gestión para controlar las llamadas sobre diferentes emergencias. Asimismo, el sistema gestiona elementos activos de emergencia (ambulancias, bomberos, policía, unidades militares, etc), la monitorización de las emergencias en curso, sistemas de video-vigilancia y comunicaciones entre los diferentes subsistemas. Se necesita acceder a los datos de las unidades activas y de las cámaras desde diferentes lugares, de manera que estos elementos del SCE puedan enviar y recibir información en tiempo real.

Las principales funcionalidades del sistema comprenden, además de la interfaz en monitores como en smartphones y tablets que lleva el personal de los sistemas activos de emergencia, un sistema de comunicaciones para gestionar las llamadas externas, las incidencias internas del sistema e identificación de llamadas entradas. Además, se cuenta con cámaras remotas que transmiten video mediante antenas de móvil/cableado urbano al sistema de emergencias.

El sistema de emergencias dispone subsistema para asignar y monitorizar distintos tipos de recursos activos para una emergencia concreta. Asimismo, un algoritmo de optimización calcula las rutas más rápidas para que las unidades lleguen al punto de emergencia. Existen distintos tipos de usuarios que gestionan y utilizan el sistema que 
son: Administrador del sistema, operarios de emergencias, operarios de unidades activas (ambulancias, helicópteros) y que reciben información en tiempo real en Smartphone/Tablet y asignadores de recursos. Las llamadas al sistema entran en una cola de llamadas permitiéndose un rango variable de entre 15 y 20 llamadas simultáneas al centro de emergencias a las cuales se identifica previamente mostrando el número en un monitor y la voz de la llamada en audio al centro de operaciones. Es necesaria una solución que distribuya las llamadas en base a los operadores disponibles en ese momento.

En algunos casos las emergencias se detectan mediante eventos inesperados que se recogen a través de sensores situados en diferentes puntos de la geografía (detectores de incendios) y que transmiten información vía radio a un centro de control remoto el cual envía un SMS y una alerta al sistema de emergencias que se procesan adecuadamente. Deberá ser posible gestionar hasta 20 eventos simultáneos en tiempo real qué, dependiendo de la prioridad de los eventos, diversos nodos funcionales del sistema podrán atender las diferentes peticiones de los usuarios si un nodo o un operador está ocupado. Asimismo, el sistema debe contar con medios para pre-asignar unidades activas disponibles para cada emergencia.

El despliegue del sistema cubre una región completa incluyendo sus ciudades y pueblos así como la línea de costa. EL sistema se coordina con las unidades de policía y Unidad Militar de Emergencias (UME), para lo cual existen comunicaciones cifradas por radio en canales concretos. El sistema permite conexiones internacionales con otros países para lo cual dispone de un módulo de traducción textual simultánea en tiempo real para emergencias que afectan a países colindantes o cercanos.

Asimismo, existen otros problemas de diseño en los que diferentes usuarios deben suscribirse de forma distribuida a noticias generadas por sucesos en tiempo real y esta información debe estar permanentemente actualizada para los diferentes tipos de usuarios. El sistema debe proporcionar una única interfaz para las posibles interfaces que gestionan diferentes usuarios de manera que el sistema sea más fácil de administrar y menos complejo. El sistema se debe diseñar para que sea escalable en cuanto a recursos, futuras conexiones vía Internet, y elementos de software adicionales que proporcionen nuevas funcionalidades. 


\section{Ejemplo de plantilla completada con los atributos capturados de una decisión.}

Para facilitar el uso de las plantillas, y que el tiempo necesario por parte de cada grupo en interpretar como manipular dichas plantillas no influya en nuestro experimento, les hemos dado como ejemplo la captura completa de una decisión.

Tabla VI.1. Ejemplo de plantilla de atributos de una decisión ya capturada.

\begin{tabular}{|c|c|c|c|}
\hline System: SCE & $\begin{array}{l}\text { ID: } \\
\text { DD-02 }\end{array}$ & $\begin{array}{l}\text { Date: } \\
12 / 10 / 2016\end{array}$ & Name: Selección del estilo arquitectónico por capas \\
\hline Description & \multicolumn{3}{|c|}{$\begin{array}{l}\text { Esta decisión separa el sistema en tres partes organizados por niveles en los } \\
\text { que una capa invoca los servicios de la capa inferior. }\end{array}$} \\
\hline Rationale & \multicolumn{3}{|c|}{$\begin{array}{l}\text { El estilo por capas favorece la separación lógica del sistema y asignar la } \\
\text { funcionalidad a diferentes capas ya que necesitamos describir la interfaz y el } \\
\text { acceso a datos separado de la lógica de negocio y no hay otro estilo que se nos } \\
\text { adapte mejor. }\end{array}$} \\
\hline Pros & \multicolumn{3}{|c|}{ El estilo por capas favorece la separación de concerns. } \\
\hline Cons & \multicolumn{3}{|c|}{$\begin{array}{l}\text { Puede introducir sobrecarga en las llamadas si hay un número excesivo de } \\
\text { capas. }\end{array}$} \\
\hline $\begin{array}{l}\text { Status: } \\
\text { Aprobada }\end{array}$ & \multicolumn{3}{|c|}{ Responsible: Autor_Captura. } \\
\hline $\begin{array}{l}\text { Alternative } \\
\text { decisions }\end{array}$ & \multicolumn{3}{|c|}{$\begin{array}{l}\text { DD-02.1: Utilizar una arquitectura sin capas. } \\
\text { DD-02.2: Utilizar una arquitectura por eventos. }\end{array}$} \\
\hline $\begin{array}{l}\text { Related to } \\
\text { requirements }\end{array}$ & \multicolumn{3}{|c|}{ RF1, RF2, RF4 } \\
\hline $\begin{array}{l}\text { Related } \\
\text { software } \\
\text { artifacts }\end{array}$ & \multicolumn{3}{|c|}{ Capas-v1.png, Clases-capa-1.png } \\
\hline Constraints & \multicolumn{3}{|c|}{$\begin{array}{l}\text { Hay un límite de tres capas para evitar sobrecargar en las comunicaciones } \\
\text { entre capas. }\end{array}$} \\
\hline $\begin{array}{l}\text { Related } \\
\text { decisions }\end{array}$ & \multicolumn{3}{|l|}{ DD-01 } \\
\hline
\end{tabular}




\section{Cuestionario para el Arquitecto Senior}

Se solicita al alumno con rol de arquitecto junior que indique ciertos datos relevantes, a través del formulario que detallamos en la tabla VI.2

Tabla VI.2. Datos complementarios basados en la experiencia del arquitecto senior.

$$
\text { Rol (ASS/ASC/ASJ): }
$$

Experiencia en la empresa (Años/Meses):

$$
\text { Rol: }
$$

ASS-1. ¿Cuáles fueron las dificultades para capturar las decisiones de diseño?

ASS-2 ¿Que plantillas para capturar ADD fueron las preferidas y por qué?

ASS-3 ¿Percibe utilidad en capturar ADD? (SI/NO)

En caso afirmativo indique ¿cuál es la utilidad principal que percibe?

ASS-4 ¿Cómo piensa que la información de ADD pueda ser utilizada?

ASS-5 ¿Por término medio, cuanto esfuerzo extra necesitó para capturar (en minutos) los siguientes ítems?

Una decisión de diseño:

Todas las decisiones de la práctica:

Una decisión con 2 alternativas y sus alternativas:

Una decisión con entre 2 y 4 alternativas y sus alternativas:

Una decisión con entre con más de 4 alternativas y sus alternativas:

Nombre y Apellidos:

Edad:

Rol (ASS/ASC/ASJ):

Experiencia en la empresa (Años/Meses):

Rol: 
Cuestionario para el Arquitecto Cognitivo: Confeccionamos un formulario para solicitar al alumno con rol de arquitecto cognitivo que indique ciertos datos relevantes, como se detalla en la tabla Tabla VI.3.

Tabla VI.3. Datos complementarios basados en la experiencia del arquitecto cognitivo.

Rol (ASS/ASC/ASJ):

Experiencia en la empresa (Años/Meses):

Rol:

ASC-1. ¿Cuántas decisiones de diseño de los ASS ha cuestionado o desafiado?

ASC-2. ¿Cuántas veces?

(a) NO estuvo de acuerdo con las decisiones tomadas por los ASS:

(b) SI estuvo de acuerdo con las decisiones tomadas por los ASS:

ASC-3. ¿Ha expresado la posibilidad de riesgos concretos de diseño para las decisiones de diseño? (SI/NO)

¿Cuántas veces?

ASC-4. ¿Cuántas veces?

Ha llegado a un consenso sobre una decisión de diseño cuestionada con los ASS:

NO ha llegado a un consenso y los ASS decidieron tomar una decisión a sabiendas de un cierto riesgo:

ASC-5. ¿Ha comprendido de manera correcta las decisiones capturadas en las plantillas? $(\mathrm{SI} / \mathrm{NO})$

En caso contrario ¿Cuantas veces ha necesitado una explicación adicional por parte de los ASS?

ASC-6. ¿Considera útil el modelo Mind 1-2?

Indique porqué:

Nombre y Apellidos:

Edad:

Rol (ASS/ASC/ASJ):

Experiencia en la empresa (Años/Meses):

Rol: 
